\title{
DIEGO COLLADO'S GRAMMAR OF THE JAPANESE LANGUAGE
}

\section{Edited and Translated by Richard L. Spear}

International Studies, East Asian Series

Research Publication, Number Nine

Center for East Asian Studies

The University OF Kansas 


\section{DIEGO COLLADO'S GRAMMAR OF THE JAPANESE LANGUAGE}

\section{Edited and Translated by \\ Richard L. Spear}

International Studies, East Asian Series

Research Publication, Number Nine

Center for East Asian Studies

The University of Kansas 
Dedicated

TO

The Memory of

Joseph K. Yamagiwa 


\section{Table of Contents}

PREFACE

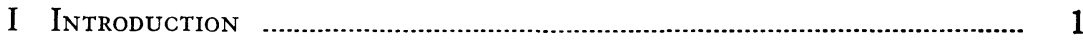

The Grammatical Framework ……….................................................... 3

The Phonological System …................................................................... 6

The Morphological System ................................................................... 8

The Structure of Collado's and Rodriguez'

Descriptions Contrasted ............................................................... 11

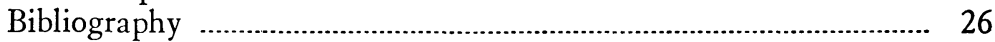

Editorial Conventions .......................................................................... 28

II Ars Grammaticae laponicae Linguae ...................................................... 1

Prologus ad Lectorem ...................................................................... 3

De nomine \& eius declinatione \& genere. ........................................ 6

De pronomine. ................................................................................ 13

De pronomine secundae [sic] personae scilicet ego $\mathcal{E} c$. .................... 13

De pronomine secundae persone: scilicet Tu, tui, tibi, Ec. ............... 14

De pronomine tertiae personae, scilicet ille, illa, illud. ...................... 15

De pronominibus relativis. .............................................................. 17

De formatione verborum, \& coniugationibus. .................................. 18

De preterito imperfecto, perfecto, \& plusquam perfecto. .................... 19

De futuro primae coniugationis. ................................................. 20

Imperativum primae coniugationis. .................................................... 21

Optativum primae coniugationis. .................................................... 21

Subiunctivum primae coniugationis affirmativae. .............................. 22

Infinitivum. ...................................................................................... 24

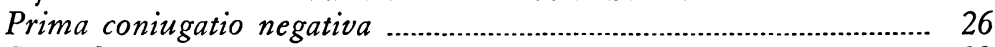

Secunda coniugatio affirmativa. ...................................................... 29

Secunda coniugatio negativa. ........................................................... 30

Tertia coniugatio affirmativa. ........................................................ 30

Tertia coniugatio negativa .................................................................. 31

Coniugatio verbi substantivi negativi. ............................................. 32

De particulis conditionalibus. ........................................................ 33

De verbo potentiali. ....................................................................... 35

Verba irregularia quo ad coniugationes. ............................................ 36

De verbo adhuc, \& de eius formatione $\mathcal{E}$ differentijs. ......................... 37

De aliquibus verbis quae de se habent honorem determinatum........ 41

Advertentiae circa coniugationes verborum. ...................................... 42

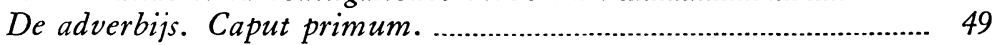

De adverbijs locorum. ................................................................... 49

Adverbia ad causam interrogandum \& respondendum. ...................... 51

Adverbia temporis. .......................................................................... 52

Adverbia negandi. ................................................................................ 53

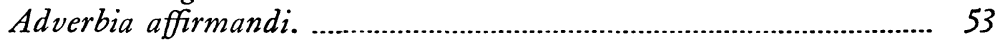

Adverbia comparativa. ................................................................... 54

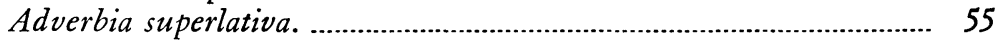

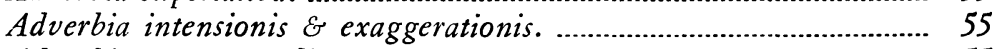

Adverbia congragendi. .................................................................... 55

Adverbia concludendi \& advertendi. ................................................. 56

De Praepositionibus casuum. .......................................................... 57

De coniunctionibus \& divisionibus. ................................................. 59 
De interiectione.

De sintaxi, \& casibus, quos regunt verba. ..................................... 61

De Arithmetica Iaponicae \& materia numerorum, in quibus hoc opus hic labor.

Aliquae regulae coniugationum in scriptura librorum. .................... 74

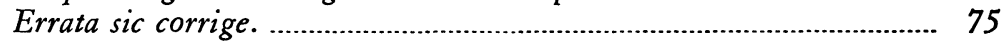

III A Grammar of the Japanese Language ............................................. 105

Prologue to the Reader ....................................................................... 107

The noun-Its Declension and its Gender ......................................... 111

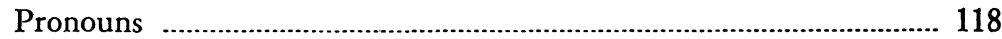

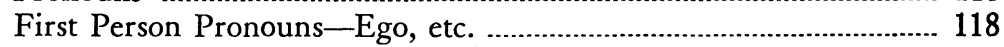

Second Person Pronouns-Tu, tui, tibi, etc. ….................................... 119

Third Person Pronouns-Ille, illa, illud. ............................................ 120

Relative Pronouns ....................................................................... 122

The Formation of the Verb and its Conjugation ............................... 123

The Preterit, Perfect, Imperfect, and Pluperfect ............................... 124

The Future of the First Conjugation ................................................. 125

The Imperative of the First Conjugation …........................................... 125

The Optative of the First Conjugation ............................................ 126

The Subjunctive of the First Affirmative Conjugation ........................... 127

The Infinitive............................................................................. 129

The First Negative Conjugation ...................................................... 131

The Second Affirmative Conjugation ................................................. 134

The Second Negative Conjugation ........................................................ 135

The Third Affirmative Conjugation .............................................. 135

The Third Negative Conjugation ................................................... 136

The Conjugation of the Negative Substantive Verb .......................... 137

The Conditional Particles ................................................................. 139

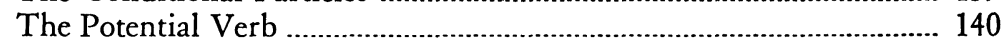

The Conjugation of Irregular Verbs ............................................... 141

The Aforementioned Verbs-Their Formation and Diversity ........ 143

Certain Verbs Which of Themselves Indicate Honor ...................... 147

Cautionary Remarks on the Conjugations of the Verb ........................ 148

The Adverbs: First Section …......................................................... 156

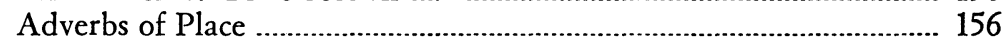

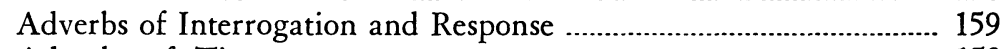

Adverbs of Time ..................................................................... 159

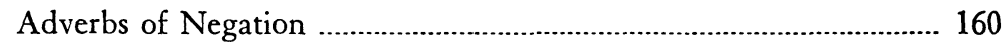

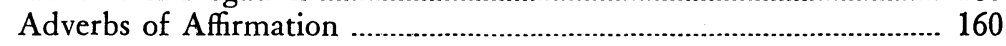

Comparative Adverbs ................................................................... 161

Superlative Adverbs ................................................................... 162

Adverbs of Intensity and Exaggeration ........................................... 162

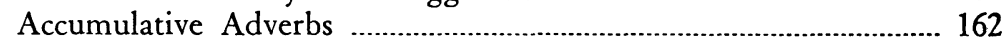

Adverbs that Conclude and Claim Attention ........................................ 163

The Case Prepositions ...................................................................... 164

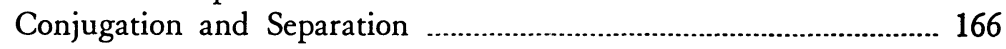

Interjections ............................................................................... 167

The Syntax and the Cases that are Governed by the Verbs ................. 168

Japanese Arithmetic and Numerical Matters Concerning

Which Much Painful Labor Is Required ....................................... 174

Some Rules on the Conjugation of the Verb

in the Written Language . 
IV Works Consulted 185

V Index to Grammatical Categories ….................................................. 187

VI Index to Grammatical Elements ......................................................... 189 


\section{Preface}

The purpose of this translation of Collado's Ars Grammaticae Iaponicae Linguae of 1632 is to make more readily available to the scholarly community an annotated version of this significant document in the history of both Japanese language study and grammatical description in general.

Collado's work, derived in all its significant features from the Arte da lingoa de Iapam completed in 1608 by João Rodriguez, is in a strict, scholarly sense less valuable than its precursor. However, if used with the Arte as a simplified restatement of the basic structure of the language, Collado's Grammar offers to the student of the Japanese language an invaluable ancillary tool for the study of the colloquial language of the early 17 th Century.

While less extensive and less carefully edited than the Arte, Collado's Grammar has much to recommend it as a document in the history of grammatical description. It is an orthodox description attempting to fit simple Japanese sentences into the framework established for Latin by the great Spanish humanist Antonio Lebrija. Thus, as an application of pre-Cartecian grammatical theory to the structure of a nonIndo-European language, the Ars Grammaticae is an important document worthy of careful examination by those wishing insight into the origins of what three centuries later was to become the purview of descriptive linguistics.

The present translation was begun with the able assistance of Ms. Roberta Galli whose contribution to my understanding of the Latin text is most gratefully acknowledged. For his continued encouragement in this undertaking I am grateful to Professor Roy Andrew Miller. Thanks are also due to the Graduate School of the University of Kansas for its support in the preparation of the manuscript and to Ms. Sue Schumock whose capable typing turned a scribbled, multi-lingual draft into a legible manuscript. The imperfections are my own.

Lawrence, Kansas

R.L.S.

May, 1975 


\section{Introduction}

In 1632, as the Christian Century in Japan was drawing swiftly to a close, three works pertaining to the Japanese language were being published at Rome by the Congregation for the Propagation of the Faith. These works were by the Spanish Dominican Father, Diego Collado (d. 1638), who had spent the years from 1619 to 1622 in Japan. Their publication clearly reflects the vitality of the missionary spirit in that age as well as the important place reserved for language study in the propagation of the faith.

The first two works, whose manuscripts had been prepared in Madrid the year before, were a grammar and a dictionary of Japanese. The third, prepared in 1631, while the larger works were being seen through the press, was a guide to the taking of confession written in both Latin and Japanese. ${ }^{1}$ The grammar, drafted in Spanish, was published in Latin in 1632 under the title Ars Grammaticae Iaponicae Linguae. It is this work that is translated here. The dictionary, only at the last moment supplied with Latin glosses to supplement those in Spanish, was published in the same year with the title Dictionarium sive Thesauri Linguae Iaponicae Compendium. ${ }^{2}$ Taken together these three works by Collado constitute the final extant efforts of those who studied the Japanese language first hand during the Christian Century. ${ }^{3}$

Two other grammatical works must be mentioned here as central to the proper assessment of Collado's Grammar. They are both by the great Jesuit scholar, Father João Rodriguez (1561-1634) $;^{4}$ the Arte da Lingoa de Iapam (Nagasaki, 1604-8, hereafter the Arte), and the Arte Breve da Lingoa Iapoa (Macao, 1620, hereafter Arte Breve). The first

\footnotetext{
${ }^{1}$ Diego Collado, O.P., Niffon no Cotoba no Yô Confesion, etc. (Rome, 1632). For further bibliographic data cf. Johannes Laures, Kirishitan Buıko (Tokyo, 1957). Cf. also Ōtsuka Mitsunobu, Koriyaado zangeroku (Tokyo, 1967), for a Japanese transliteration and concordance. It should be noted that the material in this work had no direct influence upon the concurrently written grammar. The only example in the Ars Grammaticae which might have been borrowed from the Confesion is on p. 23 where we find doco de qiqi marasuru mo, sono sata va mósanu 'although this is heard everywhere, I have heard nothing of it.' which parallels the Confesion, p. 6, 1. 18; docu [sic] de qiqi marasuru mo; sono sata ga gozaranu 'one hears about this everywhere; but, it doesn't seem to be so.'

${ }^{2}$ The bibliographical data on these and other works directly related to the study of Collado's Grammar will be found in the section on bibliography which follows.

${ }^{3}$ Other works by Collado have come down to us; cf. a memorial by him published in 1633 (Laures, Kirishitan Bunko, item 411). Such material is, however, only peripherally related to the study of language.

* For a brilliantly written biography see Michael Cooper, S.J., Rodrigues the Interpreter: An Early Jesuit in Japan and China (Tokyo, 1974).
} 
is by any standards the greatest grammatical study of Japanese made during the Christian Century. It is further, as we shall see, the primary source for Collado's Grammar. The Arte Breve, on the other hand, is not directly related to Collado's work. Indeed it is clear that Rodriguez' 1620 Macao publication was unknown to Collado. Nevertheless, since the Arte Breve is an abbreviated version of the Arte with a purpose similar to the Ars Grammaticae, a comparison of these two books with respect to the way they systematize the material from the Arte is included in this introduction to contribute some insight into the treatment of the Japanese language at the beginning of the Tokugawa Period.

In presenting this translation two potential audiences are envisioned. The first, and more restricted, group is that having an interest in the history of the Japanese language. It is hoped that an English version of this work will make more readily available this significant material pertaining to the Japanese language as spoken in the early modern period. I use the word significant here to avoid granting excessive value to a work which derives such a large portion of its material and insight from Rodriguez' Arte.

The second, and wider group for whom this translation is intended is that which has a need for an edited edition of an important document in the history of grammatical description. In this area of scholarship Collado's work is of more than moderate significance. It was accepted for publication by the prestigious Propaganda Press; and, even if those more familiar with Japanese than the editorial board of that Press might have had serious reservations concerning the linguistic accuracy of the text, it is reasonable to assume that the Press judged it to be a good example of grammatical description. It thus represents a grammar of a non-European language which suited the requirements of the day for publication at Rome. ${ }^{5}$

\footnotetext{
${ }^{5}$ The Press of the Congregation for the Propagation of the Faith was founded in 1626 when the Congregation was at the height of its activity. Grammars of the major non-European languages published during this period are:

$\begin{array}{llllll}\text { Date } & \text { Language } & \text { Grammarian } & \text { Date } & \text { Language } & \text { Grammarian } \\ 1628 & \text { Syrian } & \text { Abraham Ecchell } & 1642 & \text { Arabic } & \text { P. Guadagnoli } \\ 1630 & \text { Ethiopian } & \text { V. M. Rearino } & 1643 & \text { Georgian } & \text { F. M. Maggio } \\ 1631 & \text { Arabic } & \text { Thomas Obicini } & 1645 & \text { Armenian } & \text { Clemente Galano } \\ 1632 & \text { Japanese } & \text { Diego Collado } & 1647 & \text { Syrian } & \text { J. Acurense } \\ 1636 & \text { Coptic } & \text { A. Kircher } & 1650 & \text { Arabic } & \text { Antonio de Aguila } \\ 1637 & \text { Arabic } & \text { Germano de Silesia } & 1661 & \text { Persian } & \text { Ignazio de Jesu }\end{array}$


In order to permit this translation of the Ars Grammaticae to be of use in both these areas of scholarship I have made an effort to reduce to a minimum those places where a knowledge of either Japanese or Latin is required for the comprehension of the translation. It is sincerely hoped that the result is not an effort that is satisfying to neither, and thus to no one.

Because of the derivative nature of the text, this translation has put aside a number of important philological problems as better dealt with within the context of Rodriguez' grammars. This decision has its most obvious consequences in the section on the arithmetic, where innumerable data require exposition. However, since a basic purpose of this translation is within the context of the history of descriptive grammar, these tantalizing side roads have been left unexplored. It is, nevertheless, hoped that this translation will serve as a convenient tool for those wishing to make a more detailed investigation into the philological questions raised by the text. But I must caution those who would undertake such an inquiry that they had best begin with a careful study of the works of Father Rodriguez.

With its limitations acknowledged, the Ars Grammaticae Iaponicae Linguae remains a document worthy of our interest, and I offer this translation in order that Collado's work may more easily find its proper place in the history of descriptive grammar.

\section{The Grammatical Framework}

Collado perceived his task to be the presentation of a grammar of Japanese which would have sufficient scope to equip those dedicated to the propagation of the faith with a knowledge of the proper spoken language of his time. While he concludes his grammar with a brief, and rather presumptuous, statement concerning the written language, his purpose is clearly to train his students in the fundamentals of colloquial speech. His sensitivity to this point is demonstrated by his carefully transforming those examples presented by Rodriguez in the written language in the Arte into correct colloquial expressions in his own grammar.

The description is, of course, prescriptive. But given its age and its purpose this ought not to be construed in the contemporary, pejorative 
sense. Collado, as Rodriguez and indeed all the grammarians of the period, felt obligated to train their students in those patterns of speech which were appropriate to the most polite elements of society. Particularly as they addressed themselves to missionaries, they wished to warn them away from such illiteracies as might undermine their capacities to propagate the faith.

The description further reflects the traditional process conceptualization of language. This is particularly obvious in the treatment of the verb. Thus:

Praesens subiunctiui fit ex praesenti indicatiui mutato $\mathrm{u}$ in quo finitur in eba.... (The present subjunctive is formed from the present indicative by changing the $u$ in which it ends to $e b a . .$.$) [p. 23].$

In general each of the verbal forms is conceived to be the result of a specified alteration of a basic form. Likewise the nouns are treated within the framework of the declension of cases.

The treatment of Japanese forms is based upon a semantic framework within which the formal characteristics of the language are organized. For example, given the construction aguru coto aró (p. 31) and its gloss 'Erit hoc quod ist offere: idest offeret (It will be that he is to offer, or he will offer),' it is clear that the aguru coto is classified as an infinitive because of its semantic equivalence to offere. The same is true of the latter supine. If the form in Latin is closely associated with such constructions as 'easy to,' or 'difficult to,' the semantically similar form which appears as the element iomi in iominicui 'difficult to read,' must be classed as the latter supine. Rodriguez in his Arte Breve of 1620-unknown to Collado-makes an attempt to classify the structural units of Japanese along more formal lines; but in Collado's treatment the semantic, and for him logical and true, classes established by the formal structure of Latin constitute the theoretical framework through which the Japanese language is to be described.

Collado makes reference to two specific sources of influence upon his grammar. The first is included in the title to the first section of the grammar, Antonius Nebrissensis. It is to this great Spanish humanist, 
better known as Antonio Lebrija (1444-1522), that Collado turns for the model of his description.

An examination of Lebrija's grammar, the Introductiones Latinae (Salamanca, 1481), shows that from the basic outline of his presentation, to the organization of subsections and the selection of terminology, there is little departure by Collado from his predecessor.

Even in such stylistic devices as introducing the interrogatives by giving the form, following it with "to which one responds," and then listing a number of characteristic answers; Collado is faithful to the Introductiones.

But it is from his Jesuit colleague, Father João Rodriguez, that Collado receives his most significant influence. There is no section of his grammar that does not reflect Rodriguez' interpretation of the raw linguistic data of Japanese. On the basis of the innumerable examples taken from Rodriguez-most of the substantive sentences are directly quoted from the Arte-as well as the parallel listing of forms and identical descriptions of certain grammatical phenomena, it is clear that the writing of the Ars Grammaticae Iaponicae Linguae consisted to no small degree of abridging the exhaustive material contained in Rodriguez' grammar and arranging it within the framework of Lebrija's Introductiones.

To say that Collado followed Lebrija in the general structure of his description is not to imply that he fell heir to all of his precursor's virtues. The Salamanca grammar of 1481 is a masterpiece of orderly presentation. Printed in lettera formata with carefully indented subdivisions, it offers the student a clear display of the conjugational system as well as long columns of Latin examples of a given grammatical structure, accompanied on the right side of the page with Spanish equivalents. Collado makes little effort at copying this orderly display. There are in his presentation no paradigms, but instead only loosely connected sentences that talk the student through the various forms of the conjugation; and there is no orderly array of examples. Add to this the innumerable factual and typographical errors, and one is left with a presentation that lacks most of the basic scholarly virtues of its precursor.

A similar criticism may be leveled against the work from the point 
of view of Rodriguez' influence. Without matching the Introductiones in orderliness, the Arte more than compensates for its casual format by containing a mass of exhaustively collected and scrupulously presented linguistic data. ${ }^{6}$ There was available no better source than the Arte from which Collado might have culled his examples of Japanese.

One doubt that remains in assessing Collado's use of Rodriguez' material is that perhaps his presentation of the most readily understandable material in the Arte is not so much an effort on his part to simplify the learning of Japanese for his students, as it is a reflection of his lack of adequate familiarity with the language he was teaching.

\section{The Phonological System}

A study of the phonological data reveals the Ars Grammaticae Iaponicae Linguae to be of minimal historical value. Any student of the phonology of early modern Japanese should turn to the far more reliable work of Father Rodriguez. Nevertheless, certain aspects of Collado's transcription require our attention.

The most obvious innovation in the representation of the language is Collado's transcription with an $i$ of the palatal consonant which all his contemporaries record with a $y$. Thus in the text we find iomi and coie (terms for native words and Chinese borrowings) where Rodriguez writes yomi and coye. This change was affected while the text was being translated from the Spanish manuscript which uses $y$; and Collado himself must have felt the innovation to be of dubious value since he retained $y$ for the spellings in the Dictionarium. ${ }^{7}$

Collado's handling of the nasal sounds is too inconsistent to be a reliable source for phonological data. Given his rather awkward specification that nasalization is predictable before what we must assume he means to be the voiced stops and affricates, ${ }^{8}$ his grammar presents an uncomfortably irregular pattern in the transcription of the phenomena. Thus, on page 39 we find vo módori aró ca? as well as

'Rodriguez' own work is strongly influenced by the format found in Manuel Alvarez (1526-1582), De Institutione Grammatica, Libri III (Lisbon, 1572). So much a part of the training in the Society of Jesus was this work that an edition was printed in 1594 as one of the earliest products of the Mission Press at Amakusa.

${ }^{7}$ The palatal semi-vowel is represented, as in most the Christian materials, by a number of transcriptional devices such as $i, e, h$, and palatal consonants; e.g., fiacu, agueô, cha, and xô.

${ }^{8}$ See the translation, p. [82], n. 8 . 
modori aró ca?. Again, what he presents as the ending $z \tilde{u} b a$ in his description of the formation of the negative conditional (p. 34) appears in tovazunba in its only occurrence in a sample sentence (p. 62). To further confound the issue such forms as tovazunba and qinpen occur in contrast to sambiacu, varambe, and varãbe.

In Chart 1 the traditional pattern of the gojūonzu (chart of 50 sounds) is followed as a convenient framework in which to display the transcriptional system employed by Collado.

Chart 1

COLLADO'S TRANSCRIPTION SYSTEM

The Simple Series

/\#/ / $/ \mathrm{g} / \mathrm{g} / \mathrm{s} / \mathrm{z} / \mathrm{t} / \mathrm{d} / / \mathrm{n} / / \phi / / \mathrm{b} / \mathrm{p} / / \mathrm{m} / / \mathrm{y} / / \mathrm{r} / \mathrm{w} /$

/a/ a ca ga sa za ta da na fa ba pa ma ia $\underline{\text { ra }} \underline{\text { va }}$

/i/ $\underline{i}$ qi gui xi ji chi gi ni fi bi pi mi - $\underline{\text { ri }}$ -

/u/ ú cu gu su zu tçu zzu nu fu bu pu mu iu $\underline{\text { ru }}$ -

/e/ [ie] qe gue xe je te de ne fe be pe me ie re

/o/ [vo] co go so zo to do no fo bo po mo io ro vo

\section{The Long Series}

/au/ [vó] có gó só zó tó dó nó fó bó (pó) mó ió ró vó

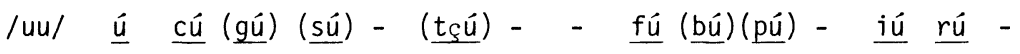

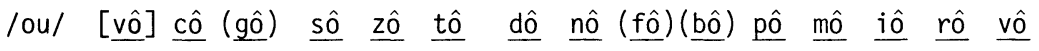

\section{The Palatal and Labial Series}

/ky/ /gy/ /sy//zy//ty/ /dy/ /ny/ / $y / /$ by/ /py/ /my/ /ry/ /kw/ /gw/

/a/ (qua) (guia) xa ja cha gia (nha) fia bia pia (mia) (ria) qua gua

/u/ qui (guiu) xu ju (chu)(giu)(nhu)(fiu) - - (miu)(riu) - -

/0/ qio guio xo (jo) cho gio (nho) (fio) (bio) - (mio) (rio) - -

/au/ qió guió xó jó chó gió - (fió) bió - mió (rió) quó guó

$/ u u /(\underline{q u u ́}$ ) (guiú) (xú) jú (chú) giú nhú - - - - (riú) - -

/ou/ qiô (guiô) xô jô chô giô nhô fiô (biô) piô (miô) (riô) - -

gueô geô neô beô reô

In this chart the phonemic grid is presented in a broad phonetic 
notation while the underlined entries are in the form used by the text. Dashes indicate sequences which do not occur in the Christian material; while the forms in parentheses are sequences which do not occur in the text but have been reconstructed on the basis of the overall system from sequences attested to elsewhere. The forms ie, vo, vó, and $v o ̂$ have been placed in brackets to indicate that neither $/ \mathrm{e} /, \mathrm{/o} /, \mathrm{loo} /$, or $/ \mathrm{au} / \mathrm{occur}$ in the syllable initial position; and, where in the modern language they do, the text regularly spells that with an initial $i$ or $v$. The forms in $e \hat{o}$ at the foot of the chart represent sequences that are phonetically identical to the forms above them, but which are transcribed differently to reflect morphological considerations; e.g., the form agueô from the stem ague. The phonetic values of /au/,/uu/, and /ou/ are [o:], [u:], and $[\mathrm{o}:]$.

Two aspects of the usage of $q$ should be noticed. First, as in the Arte, $c$ is changed to $q$ before $o$ and $u$, when the sequence occurs at a morphological juncture; e.g., ioqu 'well,' and iqó 'I shall go.' (This rule does not extend to $a$ in such contexts; cf., iocatta 'was good.') Second, in contrast to the system used by Rodriguez, Collado does not feel compelled to follow $q$ with $u$ in all contexts. Thus what Rodriguez spells as queredomo Collado spells as qeredomo. Finally, the text records one usage of the letter $h$ in the exclamation $h a$.

\section{The Morphological System}

Collado's treatment of the morphology contains one quite obvious difference from those of his predecessors: he isolates the particles of the language as separate elements of the structure. While his effort is more or less carelessly maintained by the type setter, his attempt to establish a division between the semantemes (shi) and the morphemes (ji) of Japanese by establishing formal distance between his verba and particula, reflects his consciousness that the morphological elements in Japanese are of a different order than those in Latin. At times, such as when he describes the preterit subjunctive as agueta raba, his divisions fly in the face of derivational history. But he can claim a reasonable justification for his decision by citing Rodriguez' rule for the formation of this form; "add raba to the preterit of the verb" (Arte, $18 v)$. Perhaps it is a prejudice founded upon familiarity with contem- 
porary romanizations, but I cannot help but consider this attempt to give greater independence to the particles as an improvement in the representation of the morphological system.

In all other significant facets of the morphology Collado follows the principles established by Rodriguez with the one exception that in the over-all systematization of the verbal formation and conjugation he follows the classifications established in Lebrija's Introductiones rather than those which Rodriguez inherited from the Institutiones of Alverez. The most significant difference between the two systems is the use by Lebrija of the term subjunctive in his description of the moods where Rodriguez gives independent status to the conjunctive, conditional, concessive, and potential. As we shall see, after presenting the conjugational system of the verb within the framework of Lebrija, Collado breaks the expected sequence of his description of the verb to interject a section on conditional constructions and another on those of the potential.

In the treatment of the tenses Collado breaks with Rodriguez in not attempting to establish an imperfect for Japanese, but he does follow him in the overall classification of the conjugations. Thus: ${ }^{9}$

$\begin{array}{lll}\text { 1st Conjugation } & \begin{array}{c}\text { verbs ending in } e, g i, \text { and } j i(x i \\ \text { and maraxi) }\end{array} & \text { e.g., ague, uru } \\ \text { 2nd Conjugation } & \text { verbs ending in } i & \text { e.g., iomi, } u \\ \text { 3rd Conjugation } & \text { verbs ending in } a i, o i \text {, and } u i & \text { e.g., narai, ó }\end{array}$

To the description of this general system Collado adds the treatment of the substantive verbs. This section in many respects is the weakest in his grammar with a portion of his description lost in composing the final text.

Since Collado does not, as Rodriguez, present the conjugations in paradigmatic form, I have extracted from his presentation the most representative forms of the verb ague,uru for each of the categories of the system, and presented them in Chart 2 for reference.

Chart 2

\section{THE CONJUGATIONAL SYSTEM

$$
\text { Affirmative Negative }
$$

Present

Perfect

$\begin{array}{ll}\text { aguru } & \text { aguenu } \\ \text { agueta } & \text { aguenanda }\end{array}$

${ }^{9}$ Collado's and Rodriguez' analyses agree in classifying the ni-dan verbs and suru into one conjunction, the yo-dan verbs into a second, and the ha-gyo of the yo-dan into a third. 
Pluperfect

Future

Future perfect

Present

Future

Present

Preterit

Future

Present

Perfect

Pluperfect

Future

Present

Preterit

Future

Present

Preterit

Future

Present

Future

Present

Future

Present

Preterit

Future

Present

Preterit

Future

Present

Preterit

Future aguete atta

agueôzu

aguete arǒzu

IMPERATIVE MOOD

ague io

agueôzu

aguru na

OPTATIVE MOOD

avare ague io caxi

agueôzu mono vo

avare ague io caxi

SUBJUNCTIVE MOOD

agureba

agueta reba

aguete atta reba

agueô toqi

aguru mai

aguenande atta

aguru mai

avare aguru na caxi

aguru mai mono vo

avare aguru na caxi

agueneba

aguenanda reba

aguru mai qereba

PERMISSIVE SUBJUNCTIVE MOOD

agueredomo

agueta redomo

agueôzu redomo

aguenedomo

aguenanda redomo

aguru mai qeredomo

INFINITIVE

aguru coto

agueta coto

agueô coto

aguenu coto

aguenanda coto

aguru mai coto

GERUND IN $D I$

aguru [jibun]

agueô [jibun]

aguenu [jibun]

GERUND IN DO

aguete

aguru mai [jibun]

agueĩde

GERUND IN DUM

aguru tame

agueô tame

aguenu tame

aguru mai tame

SUPINE IN TUM

ague ni

SUPINE IN TU

ague

$$
\text { PARTICIPLE }
$$

aguru fito

agueta fito

agueô fito

aguenu fito

aguenanda fito

aguru mai fito

The forms treated separately are:

THE CONDITIONAL

agueba

agueta raba

agueô naraba

THE POTENTIAL

aguru ró

aguetçu ró

agueôzu ró aguezũba

aguenanda raba

aguru mai naraba

aguenu coto mo arózu

aguenanzzu ró

aguru mai coto mo arózu 


\section{The Structure of Collado's and Rodriguez' \\ Descriptions Contrasted}

In every section of his description, Collado is indebted to the material presented by Rodriguez in his Arte da Lingoa de Iapam. The structure of the Ars Grammaticae, however, follows a much more simplistic design than that of the Arte. As a consequence Collado found it necessary to assemble his data from various sections of Rodriguez' description. In the paragraphs which follow we will briefly sketch the structural relation between these two grammars.

As he clearly states in his title to the main portion of the grammar Collado bases his description on the Introductiones of Antonio Lebriya, and more specifically upon that portion of the great Latin grammar which dealt with the parts of speech. Further, he limits himself to the spoken language rather than attempting, as does Rodriguez, an integrated treatment of both the spoken and written grammars.

Under these influences Collado's grammar takes on the following form:

A Prologue (including the phonology)

The Body of the Grammar (by parts of speech) ............................... 6-61

A Brief Syntax ........................................................................ 61-66

A Treatment of the Arithmetic .................................................... 66-74

A Note on the Written Language .................................................. 74-75

In contrast Rodriguez' Arte, prepared under the influence of Alvarez' Institutiones, develops its description over the span of three books which treat both the spoken and written grammar in progressively greater detail. Thus:

The Introduction

iii-v

Book I

The Declensions

The Conjugations

The Parts of Speech (Rudimenta)

Book II

The Syntax of the Parts of Speech

Styles, Pronunciation, Poetics, etc.

\section{Book III}

The Written Language

Names, Titles, etc.

The Arithmetic 
Given these differing formats ${ }^{10}$ it is clear that Collado is unable to cope adequately with the more complex aspects of the grammar, specifically those syntactic constructions to which Rodriguez devotes almost an entire book.

An analysis of Collado's description and a listing of the portions of Rodriguez' grammar from which material was taken yields the following:

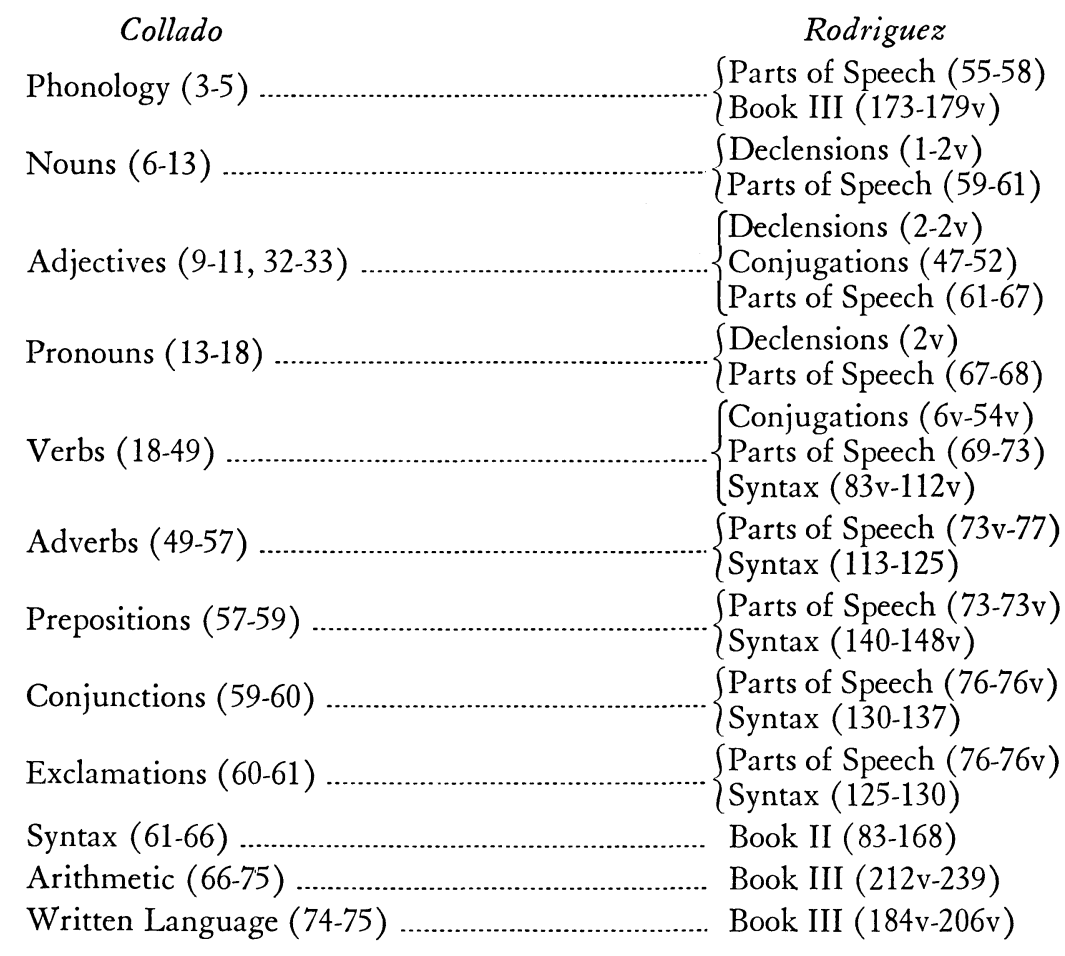

Two aspects of Japanese were not able to be described with any degree of satisfaction by Collado; the adjectives (adjectiva) and the prepositions (praepositio). His difficulties, attributable to the basic structural difference between Latin and Japanese, were compounded by the fact that Rodriguez too was unable to find a satisfactory solution to their description.

With respect to the adjectives, Collado attempts to deal with their functions in the manner appropriate to Latin, that is as a sub-class of

\footnotetext{
${ }^{10}$ It should be recalled that the Ars Grammaticae is numbered by the page and the Arte by the leaf.
} 
nouns (pp. 9-11). He also recognizes their formal similarity to the verb and treats them briefly as a sub-class of the substantive verb (pp. 32-33), but his heavy reliance upon the semantic categories of Latin does not permit him to follow Rodriguez who is able more clearly to recognize their formal as well as their functional distinctiveness.

Concerning prepositions, Collado was confronted with an all but insurmountable taxonomic problem. Here too Rodriguez was unable to develop a completely satisfactory descriptive framework. In the Arte the term posposição is used for those particles which function in a manner similar to the Latin prepositions; e.g., tameni, taixite, and tomoni (cf. $73-73 \mathrm{v}$ and $140-148 \mathrm{v}$ ); the term artigo is used for those particles having the functions of the inflectional endings of Latin; e.g., ga, ye, and ni (cf. 1-2, 78, and 137-140); and the general term particula is used to cover the broad spectrum of particles that include adverbs, conjunctions, and exclamations, as well as those otherwise unaccounted for elements which end phrases, clauses, and sentences; e.g., no, nite, and yo (cf. 77-78 and 144-154v).

Collado, rather than attempting to refine the system suggested by Rodriguez, follows the Arte in listing as praepositio those elements which translate the Latin prepositions (pp. 57-59) but uses the term particula to cover all the other particles of the language.

This tendency of Collado's to retreat from the challenging problems left unresolved by Rodriguez constitutes the greatest weakness of his description. Given concise grammatical descriptions on the one hand and over-simplified versions of previous works on the other, the Ars Grammaticae Iaponicae Linguae unfortunately falls among the latter.

In his shorter work, the Arte Breve of 1620, Rodriguez retains the same general format, but makes every effort to reduce the description to its barest essentials. Thus:

\section{Book I}

A General Note on the Language $1-2$

An Essay on How to Learn the Language ................................... 2v- 2v

The Orthography ....................................................................... $6-8$

Composition of the Syllables ……........................................... $8 \mathrm{v}-9 \mathrm{v}$

The Way to Write and Pronounce the Letters ............................ 10-12v

The Declension of Nouns ............................................................... 13-18

The Conjugation and Formation of Verbs ..................................... 18-52 
The Rudamenta

Book III

The Written Language

Of particular interest in the context of Collado's grammar is the manner in which Rodriguez displays the verbal system. While the Arts Grammaticae presents the verbal system as a series of alterational rules to be applied to the base forms, the Arte Breve goes even further than the Arte to differentiate the formational rules from the conjugational displays. Rodriguez tries several devices to elucidate his material. For example, Charts A and B below represent very early attempts to use a bordered format for linguistic description.

In order to indicate the differences to be found between the descriptions presented by Rodriguez and Collado, I have extracted the formational rules from the Arte Breve and, setting aside only two short appendices dealing with variant forms, present them here in their entirety.

\section{THE CONJUGATION AND FORMATION OF THE TENSES AND MOODS OF THE VERBS}

All the verbs of this language may be reduced (se reduzem) to four affirmative and three negative conjugations. This is because the negative conjugation of the adjectival verb, which we discussed before, ${ }^{11}$ agrees with the second of the three conjugations; and the conjugation of the substantive verb Sorrai, Sorrǒ, or soro, which is an abbreviated form of Samburai, samburơ ${ }^{12}$ both in the affirmative and the negative is reduced to the third conjugation. At this point we will treat the three affirmative and three negative ordinary conjugations of the regular personal verbs. ${ }^{13}$ Following this, and on account of its particular usage and formation, we will discuss the conjugation of the adjectival verb.

The verbs of this language do not change (naõ fẽ variedade) to show person and number as do those of Latin; rather, one form (voz)

\footnotetext{
${ }^{11}$ See p. 14, under Dos nomes adiectivos, where the initial distinction is drawn between nominal and verbal adjectives.

${ }^{12}$ Rodriguez does not treat the substantive verb in Arte Breve, but refers the reader to his earlier work for its description.

${ }^{13}$ Verbo pessoal as contrasted with verbo substantivo and verbo adjectivo.
} 
is used for all persons, singular and plural. Number and person are understood according to the subject (Naminativo [sic]), or pronoun, which is joined to the verb. The moods of the verb, which in this language have distinct forms for the tenses, are indicative, imperative, conjunctive, conditional, and preterit participle. The remaining moods are made up of these forms joined to certain particles. Each mood has but three tenses which have distinct forms; these are preterit, present, and future. These forms are signified by the Japanese terms (vocabulos) Quaco, ghenzai, mirai. The preterit imperfect and pluperfect are made up of the present, preterit, and preterit participle together with the substantive verb, as will be seen below in the conjugations.

Concerning the formation of the tenses and moods of the verbs in general, one is reminded that to understand the actual root (raiz) and the natural formation of all the tenses and moods, both affirmative and negative, it is extremely important to take notice of the usage of the Goyn, ${ }^{14}$ which are the five vowels (cinco letras vogaes) in the syllables which are below each aforementioned formation; and that it is also important to understand Canadzucai, ${ }^{15}$ which is the way to write with Firagana as well as the way one joins together syllables, or letters, to form other words (palauras), while noticing which syllable is changed by which, what constitutes long, short, or diphthongal syllables, which combinations cause contraction (sincope), which cause augmentation (incremento) of the verb, whether one makes a syllable liquid (liquescit) ${ }^{16}$ or not, and how the tenses of the moods are written with the same Cana. ${ }^{17}$ The term Goyn, not only indicates the syllables, or Cana, which are transformed to others, such as $\mathrm{Fa}, \mathrm{Fe}, \mathrm{Fi}, \mathrm{Fo}, \mathrm{Fu}$, which are changed to the closely related sounds $\mathrm{Ba}, \mathrm{Be}, \mathrm{Bi}, \mathrm{Bo}, \mathrm{Bu}$ and $\mathrm{Pa}, \mathrm{Pe}, \mathrm{Pi}, \mathrm{Po}, \mathrm{Pu} ;$ but it also indicates another kind of change from one sound to another in the same order (ordem), as happens among the syllables $\mathrm{Fa}, \mathrm{Fe}, \mathrm{Fi}, \mathrm{Fo}, \mathrm{Fu}$. Ba, Be, Bi, Bo, Bu. Ma, Me, Mi, Mo, Mu,

\footnotetext{
${ }^{14}$ Rodriguez defines this term elsewhere $($ Arte, 56$)$ as the vowels, $A, I, V, Y e, V o$, in that order. See also the introduction to the Vocabulario.

${ }^{15}$ This term, not found in the Arte, is applied. to the entire complex of "spelling" rules which Rodriguez introduces into his description. While no clear-cut influences can be established, it is generally held by Doi and others that these rules are based upon Kanazukai no chikamichi or some similar work. See Kokugogaku taikei, Vol. 9 (Tokyo, 1964), pp. 69-77.

${ }^{16}$ Latin liquesco, "to become fluid, or melt." Used here as a term to describe the palatal and labial series.

${ }^{17}$ This last phrase is to be understood in the context of the following passages which deal with euphonic change in the absence of a devise, nigori ten, to show voicing.
} 
etc. where often by rule (regna) $\mathrm{Ma}$ is changed to $\mathrm{Mi}$; or to the contrary $\mathrm{Bu}$ to $\mathrm{Ba}$ and $\mathrm{Bi}$ to $\mathrm{Ba}$, and likewise for others. The greater part of the formation of the tenses of each mood is confined to such changes, as is clearly seen in the way one writes the tense forms with Cana. It is to this that another change belongs. That which exists among those syllables having a certain relationship and rapport between them, as $\mathrm{Ma}, \mathrm{Fa}, \mathrm{Ba}, \mathrm{Pa}$; Me, Fe, Be, Pe; Mi, Fi, Bi, Pi; Mo, Fo, Bo, Po; $\mathrm{Mu}, \mathrm{Fu}, \mathrm{Bu}, \mathrm{Pu}$; with $\mathrm{Mu}$ and $\mathrm{V}$. Thus, what is written Vma in Cana is written Muma, and Mume written for Vme in order to conform more closely to its pronunciation. ${ }^{18}$ Also $\mathrm{Mu}$ is written for $\mathrm{Bu}^{19}$ so that all the harmony (armonia) in the formations of this language are contained in the rules for Goyn and Canadzucai. Those who are informed see, as native speakers, how the tenses are formed for any mood, and which letter, or syllable, must be changed to another to affect a formation. Concerning this matter there is a booklet ${ }^{20}$ which teaches Canaduzcai, and the general rules on the subject. Teachers should have this booklet to teach more easily and advantageously those students who are learning Cana. Lacking a knowledge of Goyn and Canadzucai, some of the rules which until now have been used in the formation of verbs (some of which I have let remain as they were), are not the original and natural rules as are the Goyn. ${ }^{21}$ They are rather devices, some forming affirmative tenses and moods from negative forms and others forming them from yet other more remote sources, which appear to correspond to formational rules, but for which the proper rules are not known. The fact is that the affirmative as well as negative are formed from the affirmative, beginning with the root, as will be seen below.

Speaking in general of the formation of the verb, the forms of the indicative and imperative moods of all three conjugations are formed from the root of the verb. The rest of the tenses in the other affirmative moods are formed from either the indicative or imperative forms. In the same way, the negative indicative present is formed from the root of the verb and the other tenses of the indicative are formed from

\footnotetext{
${ }^{18}$ Rodriguez used Vma regularly in the Arte, but notes the variant Muma on $178 \mathrm{v}$.

${ }^{19}$ Presumably a reference to such variants as Samurô for Saburô.

${ }^{20}$ Liurinho, presumably a treatise such as the Kanazukai no chikamichi, by Ichijō Kanera.

${ }^{21}$ In this passage Rodriguez is suggesting that certain European grammarians, out of ignorance of native grammatical theory, have misinterpreted the formational rules; and that, perhaps for pedogogical convenience, he has retained some of these "unnatural" rules in his description.
} 
the present form. The other negative moods are formed from the indicative forms.

Formation of the Tenses for the Indicative and Imperative Moods of the Verbs of the First Affirmative Conjugation

The final syllables of the roots of the first affirmative conjugation, by which the verbs conjugated here are known, and from which the tenses of the indicative will be formed, end in $\mathrm{E}$, with the exception of the verb "to do," $\mathrm{Xi}$, or $\mathrm{Ii}$, with its compounds and certain other verbs which end in I. The verbs which belong to the first conjugation, are as follows [in Charts $A \& B$ ].

The verb $\mathrm{Xi}$ "to do," with its compounds ending in $\mathrm{Xi}$ or $\mathrm{Ii}$, follows the formation of the verbs of the first conjugation. Ii is $\mathrm{Xi}$ which has been changed (alterado) to Ii because it follows the letter N. Xi conforms to the rules for the syllables which are changed (se mudam) to others. Thus:

\begin{tabular}{|c|c|c|}
\hline $\mathrm{Xi}_{\mathrm{i}}$ & $\begin{array}{l}\text { In the present change } \mathrm{Xi} \text { to } \\
\text { Suru. In the preterit add }\end{array}$ & $\begin{array}{l}\text { Suru, xita xeô, ôzu, ôzuru, } \\
\text { xeyo, xenu, or zu. }\end{array}$ \\
\hline Faixi & $\begin{array}{l}\text { Ta to the root. In the future } \\
\text { change } \mathrm{Xi} \text { to } \mathrm{Xe} \text {. In the }\end{array}$ & $\begin{array}{l}\text { Faisuru, faixita, faixeô, } \\
\text { faixeyo, faixenu. }\end{array}$ \\
\hline Tayxi ${ }^{22}$ & $\begin{array}{l}\text { imperative change } \mathrm{Xi} \text { to } \mathrm{Xe} \\
\text { and add } \mathrm{Yo}, \mathrm{i} \text {, or sai. In }\end{array}$ & $\begin{array}{l}\text { Tassuru, taxxita, taxxeò, } \\
\text { taxxeyo, taxxenu. }\end{array}$ \\
\hline Gaxxi & $\begin{array}{l}\text { the negative add } \mathrm{Nu} \text {, or } \mathrm{zu} \\
\text { to } \mathrm{Xe} \text {. }\end{array}$ & $\begin{array}{l}\text { Gassuru, gaxxita, gaxxeô, } \\
\text { gaxxeyo, gaxxenu. }\end{array}$ \\
\hline Zonji & $\begin{array}{l}\text { In the present } \mathrm{Ii} \text { is changed } \\
\text { to Zuru. In the preterit } \mathrm{Ta} \\
\text { is added to the root. In the }\end{array}$ & $\begin{array}{l}\text { Zonzuru, zonjita, zonjeô, } \\
\text { ôzu, ôzuru, zonjeyo, } \\
\text { zonjenu. }\end{array}$ \\
\hline Caronji & $\begin{array}{l}\text { future Ii is changed to Ieô, } \\
\text { etc. }\end{array}$ & $\begin{array}{l}\text { Caronzuru, caronjita, } \\
\text { etc. }\end{array}$ \\
\hline Vomonji & & Vomonzuru, vomonjita. \\
\hline Sanji & & Sanzuru, sanjita. \\
\hline Goranji & & Goranzuru, goranjita. \\
\hline Soranji & & Soranzuru, soranjita. \\
\hline & & Canzuru, canjita. \\
\hline Manji & & Manzuru, manjita. \\
\hline
\end{tabular}

Many of these verbs have another, less used, form made by adding $\mathrm{Ru}$ to the root; e.g., Abi, abiru; Mochiy, mochiyru; xiy, xiyru. Among these are some that have only this second form and lack the first; e.g., $\mathrm{Mi}$, miru; Ni, niru; Fi, firu; Cagammi, cagammiru; Ki, kiru "to dress," as distinct from $\mathrm{Ki}$, kuru "to come"; and $\mathrm{y}$, yru.

\footnotetext{
${ }^{22}$ Read Taxxi.
} 


\section{Chart A}

[The Formation of First Conjugation Verbs Ending in $E$ ]

\begin{tabular}{|c|c|c|c|c|c|c|c|}
\hline Syllables & Roots & Formation & Present & Preterit & Future & Imperative & Negative \\
\hline $\mathrm{Te}$, & $\begin{array}{c}\text { Tate, } \\
\cdot \\
\text { Fate, }\end{array}$ & $\begin{array}{l}\text { In the present } \\
\text { change Te to } \\
\text { Tçuru. The } \\
\text { remainder are } \\
\text { from the root. } \\
\text { See above. }\end{array}$ & $\begin{array}{l}\text { Tatçuru. } \\
\text { Fatçuru. }\end{array}$ & $\begin{array}{l}\text { Tateta. } \\
\text { Fateta. }\end{array}$ & $\begin{array}{l}\text { Tatê̂, } \hat{-}- \\
\text { zu, ôzuru. } \\
\text { Fateô, } \hat{o}- \\
\text { zu, ôzuru. }\end{array}$ & $\begin{array}{l}\text { Tateyo. } \\
\text { Tatei, } \\
\text { tatesay.* } \\
\text { Fateyo, } \\
\text { etc. }\end{array}$ & $\begin{array}{l}\text { Tatenu, } \\
\text { or, zu. } \\
\text { Fatenu, } \\
\text { or, zu. }\end{array}$ \\
\hline Ie, & Maje, & $\begin{array}{l}\text { Change Ie to } \\
\text { Zuru in the } \\
\text { present. The } \\
\text { remainder are } \\
\text { from the root. } \\
\text { See above. }\end{array}$ & Mazuru. & Majeta. & Majeô. & $\begin{array}{l}\text { Majeyo, } \\
\text { etc. }\end{array}$ & $\begin{array}{l}\text { Majenu, } \\
\text { or, zu. }\end{array}$ \\
\hline $\mathrm{Xe}$ & $\begin{array}{l}\text { Saxe, } \\
\text { Mairaxe, }\end{array}$ & $\begin{array}{l}\text { In the present } \\
\text { change Xe to } \\
\text { Suru. The re- } \\
\text { mainder are } \\
\text { from the root. } \\
\text { See above. }\end{array}$ & $\begin{array}{l}\text { Sasuru. } \\
\text { Mairasuru. }\end{array}$ & $\begin{array}{l}\text { Saxeta. } \\
\text { Maira- } \\
\text { xeta. }\end{array}$ & $\begin{array}{l}\text { Saxeô. } \\
\text { Maira- } \\
\text { xeô. }\end{array}$ & $\begin{array}{l}\text { Saxeyo. } \\
\text { Maira- } \\
\text { xeyo, etc. }\end{array}$ & $\begin{array}{l}\text { Saxenu, } \\
\text { or, zu. } \\
\text { Maira- } \\
\text { xenu, or, } \\
\text { zu. }\end{array}$ \\
\hline
\end{tabular}

* Read tatesai. The punctuation Tateyo. Tatei, tatesai, is in all likelihood a typesetter's error for Tateyo, tatei, tatesai. 
Chart B

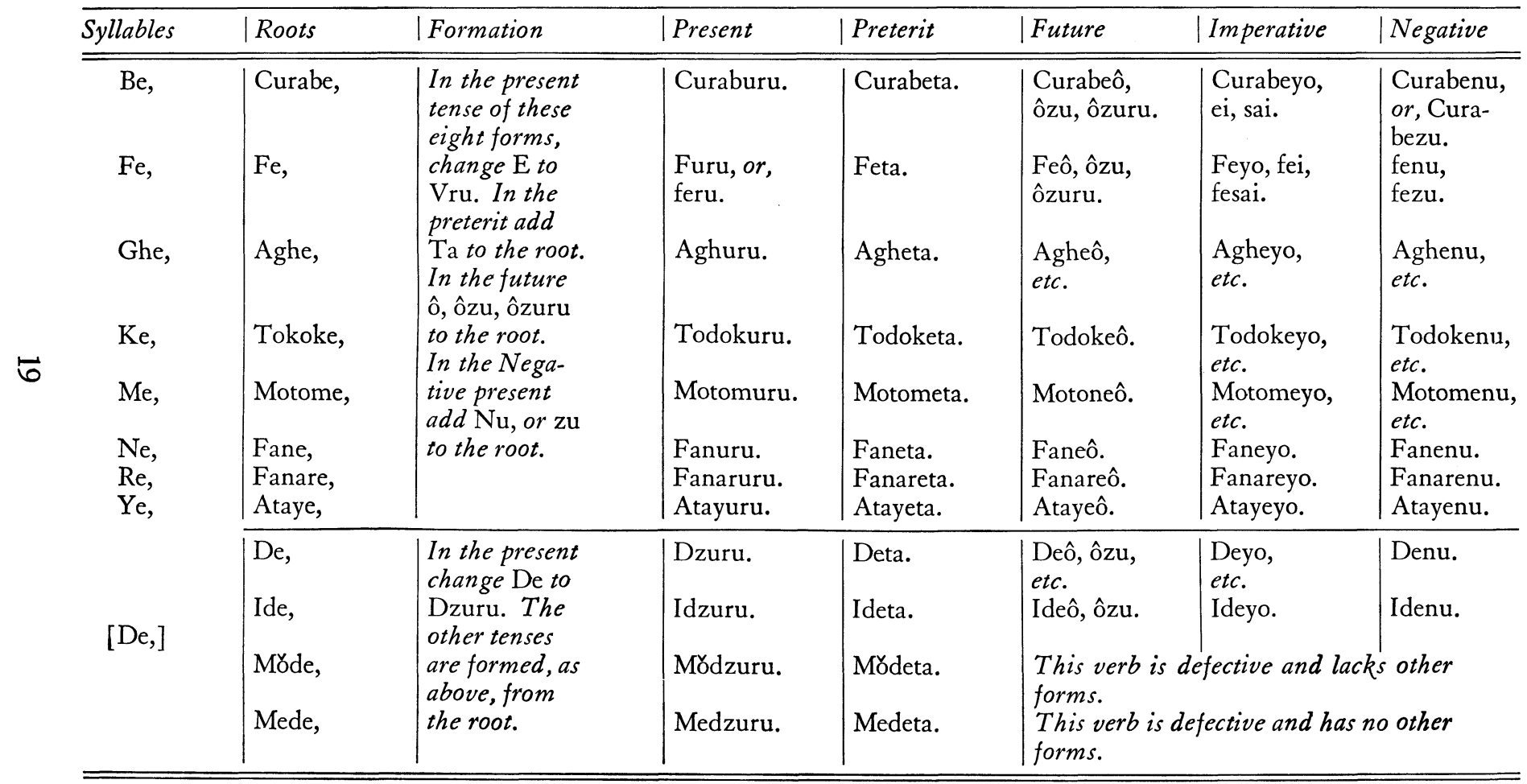




\section{Formation of the Optative, Conjuctive, and Conditional \\ Moods, and the Participle}

The optative mood does not have forms of its own but compensates for this in part by adding to the imperative certain particles which indicate desire, in part by adding to the future indicative particles which show regret for not doing something, and in part by circumlocutions with the conditional mood and certain particles, as will be seen in the conjugations.

The conjunctive mood has two sorts of proper forms. The first is the common and ordinary form ending in Eba, corresponding to the Latin cum. The other ends in Domo, corresponding to the particle "although (posto que)." The other verbs of this mood do not have their own forms, but are expressed by circumlocutions as we shall see. ${ }^{23}$

The present tense of the first conjunctive is formed from the present indicative by changing the final $\mathrm{Ru}$ to Reba; e.g., Motomureba. For the preterit Reba is added to the preterit indicative; e.g., Motometareba. For the future the final $\mathrm{Ru}$ of the third form of the future indicative is changed to Reba; e.g., Motomeôzureba. For a second form of the future the syllable Rov is added to the indicative preterit perfect; e.g., Motometaro. This particle is Ran in the written language; e.g., Motometaran. ${ }^{24}$ An utterance (oraçam) does not end in this form, but must be followed by a noun. ${ }^{25}$

The present tense of the second conjunctive is formed by changing the final $\mathrm{Ru}$ of the present indicative to Redomo; e.g., Motomuredomo. For the preterit Redomo is added to the indicative preterit perfect; e.g., Motometaredomo. Strictly speaking this form is Motomete aredomo, losing the $\mathrm{E}$ of the participle. Furthermore, Motometa, together with the other preterit forms in Ta is from Motometearu which is first elided to Motometaru and then by common usage (pratica) to Motometa. All of which is seen in its Canadzucai. For the future, the final $\mathrm{Ru}$ of the future indicative is changed to Redomo; e.g., Motomeôzuredomo.

The conditional mood, for the present tense, is formed by adding the syllable $\mathrm{Ba}$ to the root of the verb and Naraba or $\mathrm{Ni}$ voiteua to the

\footnotetext{
${ }^{23}$ The conjugational display $(27 \mathrm{v})$ lists motomuruni and motomurutocoroni.

${ }^{24}$ Rodriguez is here confusing the usage of the classical particle ran, ramu with the construction te + ara $+m u$.

${ }^{25}$ In the conjugational charts we find:

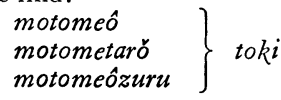


present tense form; e.g., Motomeba, motomuru naraba, and motomuruni voiteua. For the preterit, Raba, Naraba, or Ni voiteua are added to the indicative preterit; e.g., Motometaraba, which is in reality Motomete araba, motometa naraba, and motometani voiteua. For the future Naraba or $\mathrm{Ni}$ voiteua are added to the future forms; e.g., Motomeô naraba and motomeôni voiteua. The present tense forms are also used for the future.

\section{Verbs of the First Conjugation That End in $I$}

There are some irregular verbs ending in I which follow the formational rules of the first conjugation, both affirmative and negative. There are a precise number of them. Those which have been found to date are shown below. They are formed for the present indicative by changing I to Uru, for the preterit by adding Ta to the root of the verb,

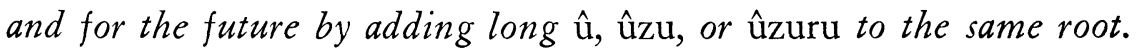
For the present conditional $\mathrm{Ba}$ is added to the root, for the preterit $\mathrm{Raba}$ is added to the preterit indicative, and for the future Naraba is added to the future indicative. For the present conjunctive the $\mathrm{Ru}$ of the present indicative is changed to Reba, for the preterit Reba is added to the same preterit indicative, and for the future the final $\mathrm{Ru}$ of the future is changed to Reba. All the other forms are formed as has been stated for the formation of the first conjugation. Thus: ${ }^{26}$

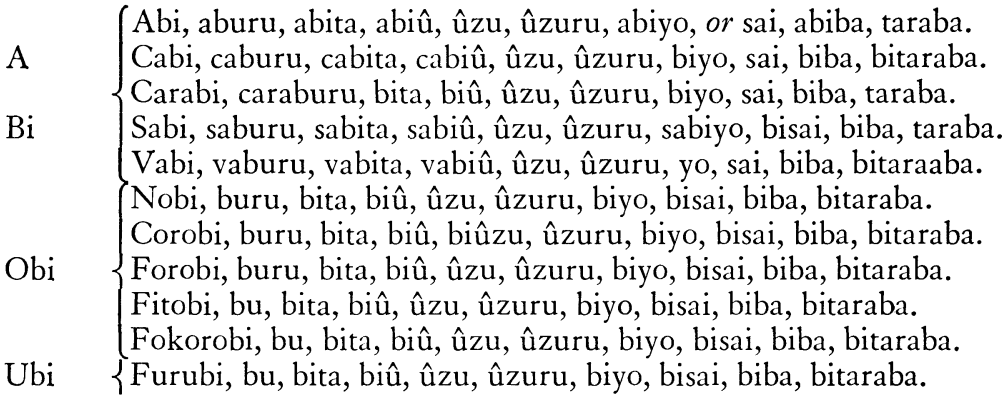

${ }^{28}$ The following notes are necessary to correct the printer's errors that occur in this listing:

a. In the perfect conditional of Nobi read bitaraba for bitaraaba.

b. The form Fitobi should in all likelihood read Fotobi 'to be wet.'

c. The forms Fotobi, Fokorobi, and Fusubi are all given present indicatives in bu. There seems to be no reason for the ending appropriate to the classical shishikei to be used for these particular verbs and the $b u$ is taken as a misprint of buru. The Arte (28) lists these forms as regular.

d. In the perfect conditional of Mochiy read ytaraba for yttaraba.

e. The form Cortu should read Cori.

f. It will be noticed in the final segment of this listing, beginning with $Y$, Rodriguez makes no effort to distinguish among Kami-ichidan, kami-nidan, and the irregular verb $K i$ 'to come.' 


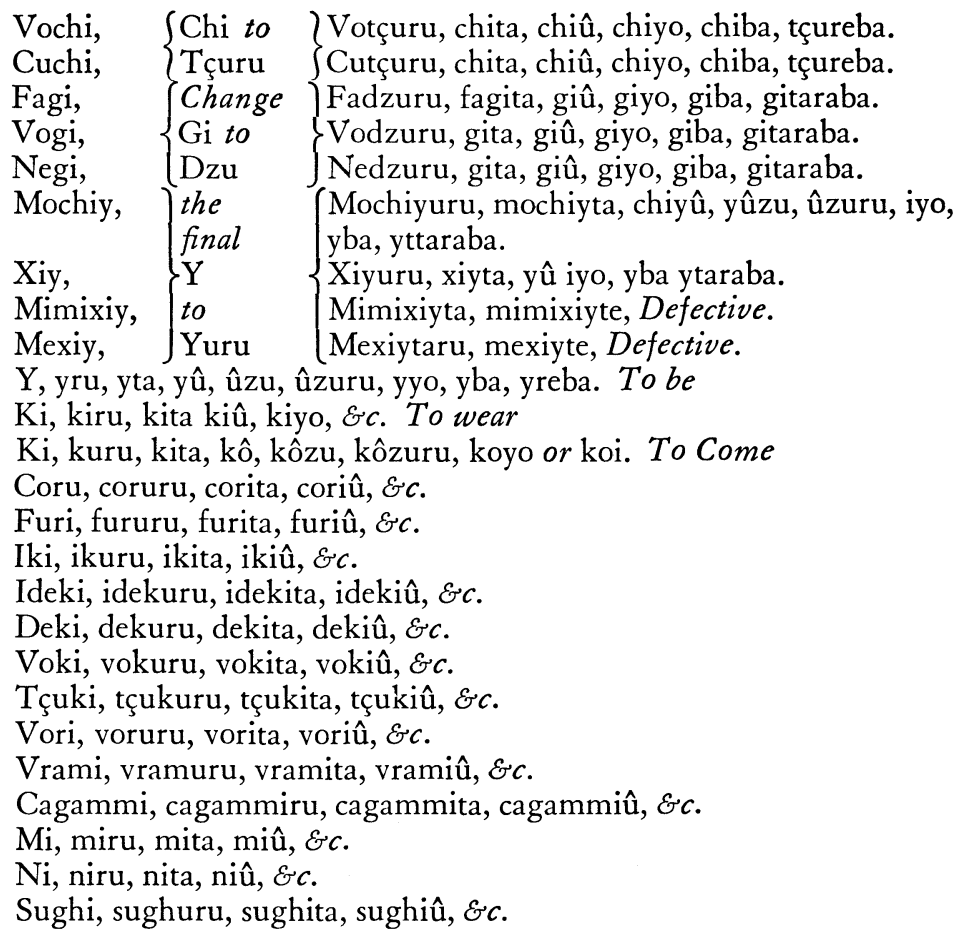

\section{Formation of the Verbs of the Second Conjugation}

All the roots of second conjugation verbs end in $\mathrm{I}$. There are eight final syllables for these verbs; i.e., $\mathrm{Bi}, \mathrm{Chi}, \mathrm{Ghi}, \mathrm{Ki}, \mathrm{Mi}, \mathrm{Ni}, \mathrm{Ri}, \mathrm{Xi}$. It is by these syllables that the verbs of the second conjugation (except for those mentioned above as being in the first conjugation) are recognized, and from which the tenses are formed.

The roots ending in the syllables $\mathrm{Bi}, \mathrm{Ghi}, \mathrm{Ki}, \mathrm{Mi}$, and $\mathrm{Ri}$ change the I to V for the present tense; e.g., Tobi, tobu; Coghi, coghu; Caki, caku; Yomi, yomu; Kiri, kiru.

Those ending in Chi change to Tçu for the present; e.g., Mochi, motçu; Cachi, catçu; Tachi, tatçu.

Those ending in $\mathrm{Ni}$ change to Nuru for the present; e.g., Xini, xinuru; Yni, ynuru. ${ }^{27}$

\footnotetext{
${ }^{27}$ By this single rule Rodriguez brings the two na-hen verbs into the second conjugation.
} 
Those ending in $\mathrm{Xi}$ change to Su for the present; e.g., Fanaxi, fanasu; Cudaxi, cudasu; Taraxi, tarasu.

For the preterit those ending Obi and Omi change to ôda; e.g., Yomi, yôda; Tobi, tôda; Yobi, yôda; Yorocobi, yorocôda. Tomi becomes tonda.

Those ending in Abi and Ami change to ǒda; e.g., Yerabi, yerǒda; Vogami, vogǒda; Yami, yǒda.

Those ending in Imi change to ûda; e.g., Najimi, najǔda; Nijimi, nijǔda; Ximi, xûda. ${ }^{28}$

Those ending in Umi and Ubi change their endings to Vnda or in some instances ûda. While some have two forms others have only one form which is seen in use, the more general is Vnda; e.g., Musubi, musunda; Susumi, susunda or susûda; Nusumi, nusunda or nusûda; Sumi, sunda or sûda; Cumi, cunda only.

Those ending in Ebi and Emi change to Eôda; e.g., Sakebi, sakeôda; Sonemi, soneôda.

Those ending in Ghi change to Ida; e.g., Auoghi, auoida; Voyoghi, voyoida; Coghi, coida.

Those ending in $\mathrm{Ni}$ change to Inda; e.g., Xini, xinda; Yni, ynda.

Those ending in Chi and $\mathrm{Ri}$ change to Tta; e.g., Machi, matta; Cachi, catta; Tachi, tatta; Kiri, kitta; Chiri, chitta; Cari, catta.

Those ending in $\mathrm{Ki}$ and $\mathrm{Xi}$ change to Ita; e.g., Caki, caita; Faki, faita; Nuki, nuita; Todoki, todoita; Sosoki, sosoita; Saxi, saita; Fataxi, fataita; Maxi, maita or maxita; Coxi, coita or coxita. The following add Ta to the root; e.g., Moxi, moxita; Muxi, muxita; Fuxi, fuxita; Mexi, mexita.

The future can be formed in two ways. The first and more common way is to change I to ǒ, ǒzu, or ǒzuru; e.g., Yomi, yomǒ, yomǒzu, yomǒzuru; Yerabi, yerabǒ, etc.; Kiri, kirǒ; Xini, xinǒ; Auoghi, auogǒ. ${ }^{29}$ Those ending in Chi change to Tǒ; e.g., Cachi, catǒ, etc.; machi, matǒ. Those ending in $\mathrm{Xi}$ change to Sǒ; e.g., Mǒxi, mǒsǒ; Nagaxi, nagasǒ; Mexi, meš̌; Coxi, cosǒ, etc. The other way, which is easy too, is to change the final $\mathrm{V}$ of the present indicative to ǒ; e.g., Yomu, yomǒ; Kiku, kikǒ; Mǒsu, mǒsǒ; Mesu, meš̌. Those ending in

\footnotetext{
${ }^{28}$ Read najûda and nijûda.

${ }_{29}$ Although the spelling auoghó would contain a redundancy it would agree with such forms as aghuru, coghanu and coghŏ found elsewhere.
} 
Tçu change Tǒ; e.g., Tatçu, tatǒ; Catçu, catǒ. Those ending in Nuru change to Nǒ; e.g., Xinuru, xinǒ; Ynuru, ynǒ. This second rule seems to be more naturally in accord with the rules for the Japanese language.

The imperative changes the final I of the root to $\mathrm{E}$. Those ending in Chi change to Te; e.g., Yome; Kike; Tamochi, tamote; Vchi, ute; Machi, mate.

The present conjunctive is formed by adding Ba to the imperative; e.g., Yomeba; Tateba. ${ }^{30}$ For the preterit, Reba is added to the preterit indicative; e.g., Yôdarebe. ${ }^{31}$ For the future the final Ru of the future indicative is changed to Reba; e.g., Yomǒzureba. The conjunctive in Domo is formed in the same manner; e.g., Yomedomo, yôdaredomo, yomorzuredomo.

The conditional is formed from the future indicative by changing the or to Aba; e.g., Yomaba; Tataba. ${ }^{32}$ The preterit is formed by adding Raba to the indicative preterit; e.g., Yôdaraba; Tattaraba. ${ }^{33}$

The preterit participle is formed from the preterit by changing the A to E; e.g., Yôde; Kite; Tatte. The present participle, in Te, is formed by adding $\mathrm{Te}$ ['hand'] to the root of any verb. This is properly a substantive and thus governs the genative as do the other substantives. It does not indicate tense; e.g., Yomite; Cakite; Machite, etc.

The negative present can be formed in two ways. The first, and that which accords with the rules for Canadzucai, is formed by changing I of the root to Anu or Azu; e.g., Corobi, corobanu, corobazu; Yomi, yomanu, etc.; Coghi, coghanu; Caki, cacanu; ${ }^{34}$ Kiri, kiranu; ini, inanu. Those ending in Chi change to Tanu; e.g., Tachi, tatanu. Those ending in Xi change to Sanu; e.g., Fanasanu. Another formation common to all is made with the future indicative by changing or to Anu or Azu; e.g., Corobǒ, corobanu, corobazu; Yomǒ, yomanu, etc.; Coghǒ, coghanu; Cakǒ, cakanu; Kirǒ, kiranu; Inǒ, inanu; Tatǒ, tatanu; Matǒ, matanu; Fanasǒ, fanasanu. This rule is common to all three conjugations by changing the affirmative future indicative or to Anu and the $\hat{o}$

\footnotetext{
${ }^{30}$ This use of the imperative reflects a purely formal solution to the morphological problem.

${ }^{31}$ Read Yôdarcba.

${ }^{32}$ This rule, which consciously or unconsciously associates the future and the conditional, is also applied to the third conjugation, while the first conjugation uses the root.

${ }^{33}$ The future is the same as the present. below.

${ }^{34}$ This spelling of the final root consonant with a $c$ is irregular for verbs. Cf. cakanu just
} 
and $\hat{\mathrm{u}}$ to $\mathrm{Nu}$ or $\mathrm{Zu} ;^{35}$ e.g., Todomeô, todomenu, todomezu; Saxeô, saxenu, etc.; Tateô, tatenu; Miû, minu; Yomǒ, yomanu; Tatǒ, tatanu; Fanasǒ, fanasanu; Narauǒ, narananu; Vomouǒ, vomouanu; Furuuo, furuuanu. For the second conjugation preterit, those in $\mathrm{Nu}$ are changed to Nanda; e.g., Yomananda. For the preterit participle Da is changed to De; e.g., Yomanande. For the second form of the negative participle, the $\mathrm{Nu}$ is changed to Ide; e.g., Yomaide, Corobaide, Tataide, Totonouaide. For the future the particle majij ${ }^{36}$ or mai is added to the affirmative present indicative; e.g., Yomumajij, yomumai; Matçumajij, matçumai.

\section{Formation of the Third Conjugation and the Roots from Which the Tenses Are Formed}

The final syllables of the third conjugation are the diphthongs Ai, $\mathrm{Oi}, \mathrm{Vi}$. By these syllables the verbs are known to belong to this conjugation, and from them the tenses are formed. The present indicative is formed by changing Ai to ǒ, Oi to ô, and Vi to û ; e.g., Narai, narǒ; Vomoi, vomô; furui, furû. The preterit is formed by adding the syllable Ta to the present; e.g., Narǒta, Vomôta, Furûta. The future is formed by changing the final I of the root to Vǒ, vǒzu, vǒzuru; e.g., Narauor, narauǒzu, etc.; Vomouǒ, vomouǒzu, etc.; Furuuǒ, furuuǒzu, etc. The present imperative is formed by changing the final I to Ye; e.g., Naraye, Vomoye, Furuye.

For the present conjunctive $\mathrm{Ba}$ or Domo is added to the imperative; e.g., Narayeba, narayedomo; Vomoyeba, vomoyedomo; Furuyeba, furuyedomo. For the preterit Reba or Redamo ${ }^{37}$ is added to the indicative preterit; e.g., Narǒtareba, narǒtaredomo; Vomôtareba; vomôtaredomo; Furûtareba, furûtaredomo.

The present conditional is formed by changing or of the future to Aba; e.g., Narauaba, Vomouaba, Furuuaba. The preterit is formed by adding Raba to the indicative preterit; e.g., Narǒtaraba, Vomôtaraba, Furûtaraba.

\footnotetext{
${ }^{35}$ The association of the negative with the future, and by extension with the conditional, suggests a keen awareness of the underlying system, particularly since the Canadzucai rules to which he refers require the formation be made from the present. It should be noted that this rule is significantly more elegant than that which derives the negative from the root.

${ }^{36}$ The $i j$ in the original is the digraph $i j$, as elsewhere.

${ }^{37}$ Read Redomo.
} 
The negative present is formed by changing the I of the root to Vanu or vazu; e.g., Narai, narauanu, narauazu; Vomoi, vomouanu, etc.; Furui, Furuuana, etc. This form can also be formed from the future by changing the or to Anu or azu; e.g., Narauor, narananu, etc. For the preterit the $\mathrm{Nu}$ is changed to Nanda; e.g., Narauananda. For the preterit participle the $\mathrm{Da}$ is changed to De; e.g., Narauanande. For the second form the $\mathrm{Nu}$ of the present is changed to Ide; e.g., Narauaide, Vomouaide, Furuuaide. For the future the particle Majii, ${ }^{38}$ maji, or mai is added to the affirmative present indicative; e.g., Narǒmajii narǒmaji, narǒmai; Vomômajii, ji, or mai; Furûmajii, ji, or mai.

The verb Yy 'to speak' becomes Yû, yûta, yuǒ, yye, yuanu. Yei or yoi 'to become sick' becomes Yô, yôta, youǒ, yoye, yonanu. The substantive verb Saburai, which also belongs to this conjugation, becomes Saburǒ, saburauanu; and Šrai becomes Šrrǒ, soro, sorraite, sorraye, sorouanu.

Rodriguez follows these formational rules with a full display of all the forms of the three conjugations. In his display he, like Alvarez before him, recapitulates the appropriate rules for each form. Collado nowhere presents his conjugational system as a paradigm but does, as we shall see, include a full complement of example sentences in his description, something which Rodriguez does not do in the Arte Breve.

\section{Bibliography}

In the examination of any portion of the Christian materials certain works are indispensable. Father Johannes Laures, S.J., Kirishitan Bunko (Tokyo, 1957) remains the basic bibliographic source for the study of all sources of the Christian Century, while Hashimoto Shinkichi, Kirishitan kyōgi no kenkyū (Tokyo, 1929) and Doi Tadao, Kirishitan gogaku no kenkyü (Tokyo, 1942) serve as indespensible guides to our understanding of the linguistic aspects of the field. A later contribution to the general bibliography has been made by Fukushima Kunimichi, Kirishitan shiryō to kokugo kenkyū (Tokyo, 1973).

The basic grammatical study of the period, based upon the shomono materials, is Yuzawa Kōkichirō, Muromachi jidai gengo no kenkyū

\footnotetext{
${ }^{38} \mathrm{Majij}$ with the digraph would be more regular.
} 
(Tokyo, 1958). More closely related to the language reflected in the text is his "Amakusabon Heike monogatari no gohō," in Kyöiku ronbunsh $\bar{u}$ (no. 539, Jan. 1929). An English treatment of the grammatical system of the period is to be found in R. L. Spear, "A Grammatical Study of Esopo no Fabulas," an unpublished doctoral thesis (Michigan, 1966). The phonology has been carefully analyzed by Ōtomo Shin'ichi, Muromachi jidai no kokugo onsei no kenkyū (Tokyo, 1963), with a valuable contribution made in English by J. F. Moran, "A Commentary on the Arte Breve da Lingoa Iapao of João Rodriguez, S.J., with Particular Reference to Pronunciation," an unpublished doctoral thesis (Oxford, 1971). This latter work presents an exhaustive examination of the phonological system reflected in the Arte Breve of 1620 within the framework of Berhard Bloch's phonemic theory.

Two lexical works have been used as basic references in this translation. The Vocabulario de Lingoa de Iapam (hereafter the Vocabulario) produced by the Jesuit Mission Press at Nagasaki in the years 1603 and 04. In a carefully annotated version by Professor Doi, under the title Nippo jisho (Tokyo, 1960), this work is the most important single source for the vocabulary of the period. The second work is the Dictionarium sive Thesauri Linguae Iaponicae Compendium (hereafter the Dictionarium) which is the companion piece to the present text. This dictionary has been carefully edited and cross-referenced by Ōtsuka Mitsunobu, under the title Koriyaado Ra Su Nichi jiten (Tokyo, 1966). In this form it has served as a constant aid to the translator in the determination of the proper glosses for the lexical items in the text.

The aforementioned Arte of 1604-08 by Rodriguez, has been the single most frequently used tool in the preparation of this translation. ${ }^{39}$ As the most significant influence upon Collado's work and the source for most of his material, both theoretical and practical, I have related the two works at every point in the translation. In its Japanese version by Professor Doi, Rodorigesu Nihon daibunten (Tokyo, 1950), this work has been invaluable in gaining a clearer understanding of many of the passages which might have otherwise been obscure.

Rodriguez' Arte Breve of 1620, while having no influence upon the preparation of the Ars Grammaticae, is nevertheless of fundamental

\footnotetext{
${ }^{39}$ A photostatic copy of the entire text has been made available by Shima Shōzō, Rodorigesu Nihon daibunten (Tokyo, Bunka Shobō, 1969).
} 
importance as a work against which Collado's treatment of Japanese grammar is to be judged. This shorter grammar is as yet to be fully translated into English-Moran having limited his study to the treatment of the phonology.

With respect to the text itself I have made this translation on the basis of the facsimile edition published by the Tenri Central Library in 1972 as part of its Classica Japonica series. Ōtsuka Takanobu, Koiyaado-cho Nihongo bunten (Tokyo, 1934) and its revised edition under the title of Koriyaado Nihon bunten (Tokyo, 1957) have served as invaluable aids at every step of the translation.

Ōtsuka's second edition is of invaluable scholarly importance because it contains a cross-reference to the Spanish manuscript from which Collado prepared the printed Latin edition as well as a concordance to the Japanese vocabulary. ${ }^{40}$ This translation attempts to supplement Ōtsuka's invaluable contribution by relating the Latin text of this grammar with Rodriguez' Arte.

\section{Editorial Conventions}

The Latin matrix of the text is printed in italic letters while the Japanese is in roman. For this translation I have reversed the convention. (In footnotes where the text is quoted the style of the original is followed.) In making editorial corrections in the Japanese material the corrected version is presented in brackets with periods to indicate the general location;

\section{e.g., mairu mai queredomo [... qeredomo]}

(The only exception to this rule is the correcting of a missing open $o$, q.v.) Sentences that have been taken from the Arte are indicated by the parenthetical recording of the leaf number of the citation immediately after the sentence;

$$
\text { e.g., xô tame no chôqui gia (22) 'it is.... }
$$

Shorter sentences and specific words that in all likelihood have been taken from the Arte are not listed if they are to be found in the section elsewhere noted as being the source of the material covered. Any sig-

\footnotetext{
${ }^{40}$ Ötsuka's comparison of the Spanish manuscript with the printed version of the text suggests that many of the typographical errors found in our text are the result of material being too hastily transcribed from a more correct original while the work was being translated from Latin.
} 
nificant alteration in the form of the source is noted. Since the Arte is numbered by the leaf, $v$ is added to the number to indicate the verso.

All the corrections made by the errata (on page 75 of the text) have been applied to the text without notation unless the correction is itself in error.

The punctuation follows the text with the following exceptions;

1. In translating from Latin the English follows modern rules of punctuation.

2. Single quotes have been introduced into the text to mark glosses and translations.

3. In transcribing the Japanese citations any alteration of the original punctuation is noted.

4. The spacing of words in Japanese-a relatively casual matter in the text-has been regularized on the basis of the predominant pattern.

5. Two specific rules, based upon Collado's more or less consistent usage, are followed in the citing of verb forms:

a. In the most frequent citation of verbs, where the root form is followed by the present indicative ending, a comma is used;

\section{e.g., ari,u; ague,uru; mochi,ţu}

b. In an alternate form of citation, where the two forms are given in their entirety, a colon is used;

e.g., ari:aru; ague:aguru; mochi:motçu

Spelling and accentuation are treated in the following manner:

1. The $\Gamma$ in all instances is represented by $s$.

2. The usage of $v$ and $u$ has been regularized: the $v$ serves as the consonant; and $u$ as the vowel, semi-vowel, and orthographic symbol; e.g., vaga; wie, quan, and agueta.

3. The predictable nasalization-marked by a tilde in the texthas not been included in the translation unless the presence of nasalization is morphologically significant; e.g., tobu:tóda. 
4. The accent grave-which appears in no discernible patternis not transcribed in the translation.

5. The accent acute is used in the translation to mark the long $u[\mathrm{u}:]$ and the long, open $o[\mathrm{o:}]$, in those places where the length is marked by Collado. Since the most frequent typographical error in the text is the failure to mark the presence of these long syllables, I follow the convention of correcting the absence of this feature in the Latin text by using the inverted caret in the translation. Thus, the appearance in the translation of mósu indicates that Collado recorded the length of this word, either by an accent acute (e.g., mósu), or an inverted caret (e.g., mósu). The appearance of mǒsu indicates that he did not, and that its absence is being corrected. The form morsu in the translation is therefore the shorthand equivalent for what would more regularly be mosu [mósu].

6. The circumflex, which indicates the long, closed $\hat{o}[0:]$, is corrected as other errors by placing the corrected version of the item in brackets; e.g., roppio [roppiô]. 


\section{A $\mathrm{R} S$ \\ GRAMMATICAE \\ I A P O N I C AE \\ L I N G V AE.}

I N GRATIAM ET ADIVTORIVM eorum, qui prædicandi Euangelij caufa ad Iaponix Regnum fe voluerint conferre.

Compofita, \& Sacre de Propaganda Fide Congregationi dicata à Fr.Didaco Collado Ordinis Pradicatortum per aliquot annos in pradicto Regno Fidei Catbolica propegationis Ministro.

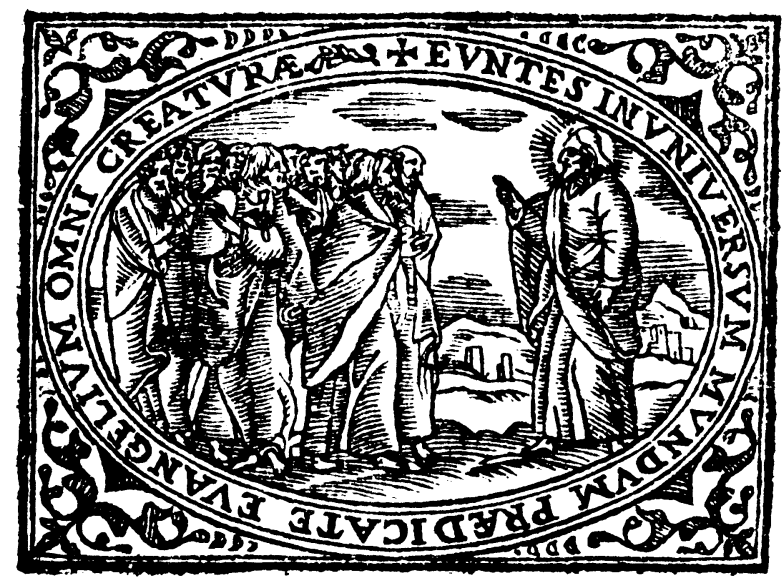

$\mathrm{R} O \mathrm{M} \nsubseteq$,

1 ypis \& impenfis Sac. Congr. de Propag. Fide. M D C X X X I I. SVPERIORVM PERMISSV. 
Imprimatur fo placet Rewerendiff. P.M.S.Pal.Apost. A. Epifc.Vmbriaticen.Vicelg.

\author{
Imprimarur
}

Fr. Nicolaus Riccardius S. Pal. Apolt. Magifter, Ordinis Prædicatorum . 


\section{PROLOGVS AD LECTOREM. Er aduertentix aliqux pro Iaponicx lingux perfecta pronuntiatione.}

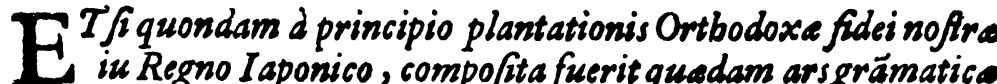
isngua pradicte a P. Ioanne Rodriguez Societatis Iefu ; quia tamen raro res in fua prima conditione perfecza lumen a picizint, o alias, ob temporum diuturnitatem, vix aliquam copiam prediefa artis eft iam inuenire: Vifum eft mibi, aliquale Deo, \& miniftris fidei propagationis (qua fine lingus infirumento non poteft efle) obfequium prafiturum , $f_{2}$ extractis ab artepradicta necel]arìs ( Sunt enim multa) \& relietis, qua peritis in prediEta lingua talia non probantur; additis etiam ijs, qua experientia, \& vvfu, beckioneque continua librorum, Deo largiente (qui dat verbum Euangelizantibus) furm adeptus, in compendium alari, $-\mathcal{S}$ Jub breuitate redigerem precepta omnia qua ad erudite vtendum lingua Iaponica, pradicatoribus (pro quibus labor It afjumptus) funt necefJaria . Quia verò fimul cum praceptis, \& regulis, expedit exempla, in quibus pradicta regula verificantur ponere; libuit etiam ad maiorem discipulorum confolationem o iutuamen non folum boc; fed etiam exemplorum omnium declarationem in lingua Latina (qua inter Tbeologos est cömanior) adiungere, quo nibil a magifiro reflet amplius defiderandum. Et $f$ iverò ea omnia, qua tam in bac arte; quam in DiEtionario ( quod, Deo dante, quanto citizus in lucem edere geftio) continentur meo inditio fint fatis limata, ov fecura, quibus fiducialiter quiuis oti potef ; Examini tamen, o iuditio melius fentientium fubiecta efje volo, ot fidei pradicatio ab eruditicre lingua, fruciuofior euadat.

Quando dua vocales immediate coniunguntur in aliquo vocabulo lingua Iaponica ; non pronunciantur ficut in Latina valeo, aut in Hifpanica vaca, Jed vtraque integra perse profertur, $\mathrm{v}, \mathrm{aj}$ $v, 0, ;, i$.

Liters, s, pronunciatur ficut, f̧̧, v.g. fufumùru, , f̧̧uf̧̧1mùru .

Litera, i, prousunciatur blande, ficut in lingua Lueftandice jo2o, , ¿ judeo.

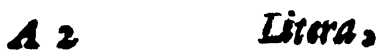




\section{P R O L O G V S}

Literax, pronasciatur etiam blandeficut in lingula Lufitanice queixumes.

Quando Jupra literam, o, fuerit boc fignum s pronunciatur, o, ac ji efjet, ou, labÿs quafi iunctis, \& ore penè claufo. v.g. búppô.

Quindo verò Supra 0 , fuerit boc fignum , v, vel, ó, pronunciatur ore aperto, of ac jiefJent dua literce, $00, v . g$. tènxó, vel gacuxǒ.

Si veropradicza figna, fuerint fupra ú, pronnntiatur ǔ , detentum, acfiefJent duo, v.g. , Tüi fu, Aiaǔ .

Quando fuerit boc/fgnum - Juper aliquă literam ex vocalibus. debet proferrificut, $\mathrm{n} ;$ fed non in integrum, Sea cungim o leniter v.g. vãga .

Qe, \& , qi, fcribuntur ab/que, u, quia quando, u, fequitı'r post, $q$, vel poft , $c$, utraque integrè pronunciatur ab $q u$ uinalepha vg. quódai, quàinin:

Quando poft, $\mathrm{g}$, fequitur, u, of pofea immediate alia vocalis c, vel, $\mathrm{i}$, pranurciatur ficut in lingua Hi/panica, v. g. guenin; ff vero litera, $\mathrm{i}$, ponatur immediate $p \circ f_{2}, \mathrm{~g}, a b f q u e, v$,pronun. ciatur ficut Italicè, giorna, , 0. g. Xitägi .

Litera, $\mathrm{z}$, pronunciatur ea vi, qua in lingua Hifpanie, Zum bar, v.g. mizu.

Si verò fuerint duo, $\mathrm{zz}$, violentius feriuntur. v.g. mizzu .

Quando fuerint duo, tt , xx, zz, qq, cq, ij , pp ; vtrumque opportet ferive vt fit perfect a pronunciatio, \& vis fignificationis percipiatur : nam v.g. mizu, fignificat mel; \& mizzu, frgnificat aquam:onde fi eadem, vel violentia, aut lenitate vtrumque pronunties vel aquam tantum, vel mel folium tibi proferent .

Quasedo, ch, anteponitur vocali , pranunciatur ficut $\mathrm{H} i \mathrm{fpa}_{\mathrm{p}-}$ nice, cbimenea . v. g. fochó.

$S_{i}$ vero, nh, anteponatur vacali, pronunciatur ficut $H i$ /panicè, maña, v.g. nhuva.

Litera, $f$, in aliquibus I apania prouincïs pronunciatur ficus in lingua Latina; in aliys astem ac fis effet, $h$, non perfectum : fed quodam medium int $r, f, \&, h$, os o labia plicando of claudendo, fed non integrum, quod vfuf acilè compertum erit : v.g. fito.

Quando, tç,in aligno vocabulo fuerit (quod eft valde frequons) orcioe debet difcipulus Deum, ot ei vereaspronuntiationis aperiat 
quera eft difficilis, ơ in nulla lingua alia, eft talem pronuntiationem inuen:re: non enim pronunciatur vt $t c ̧$, aut ut $f$, uel, $c$, folum, fed uiolenter percutiendo lingua dentes, ita ut utraque. litera, \&, $t, \mathcal{d}, c ̧, \dot{\jmath}$ plus, $c$, quàm, $t$, feriri uideantur. \#.g. tçùtçùmu.

Litera, $\mathrm{r}$, pronunciatur blandè of leniter ubicumque inueniatur, fiue fit in principio, fiue in medio uocabuli;u.g., ranguió, cutabirìru.

Ya, ye, yo, yu, pronunciantur ficut in lingua Hi/panica.

Quando a I aponïs pronunciantur uocabula finita in, $\mathrm{i}$, uel $\nabla$, uix percipitur litera finalis a ty ronibus . u.g. qui audit, gozi$\mathrm{ru}$, putat fe audiui/Je, gozdr, or qui audiuit fitòtçu, credit fe folum audiuiffe, fitòç, \& cum audit, àxino fàra, percipit Solum, ix no fira.

Quando uocabulo finito in uocali fubfequitur incipiens in confonanti, precipuej $f$ it , $b$, uel, $f$, inter ipfam uocalem, \& confonantem, pronunciatur litera $\mathrm{n}$, red non perfecta ; fed lenis. ug. lònógotòqu.

Circa uocabselorum accentus magnam adbibui curam, ut illos fignis fuis proprÿs locis fupra literas in quibus accentus fieri debent,adaptatis, fenfus of fentertia loquentis percipiatur.u.g. qei xèi , babet accentum in utroque, è̀ . fibicàxi, babet in prima, $i$, ơ in, a , o idem in dictionario feruabitur ordo, notando accentus ea perfectione, qua fumma cum diligentia potui percipere, $\sqrt{i}$ in aliquo fum deceptus, paratus fum corrigi ; ea uerò quie diminute fuerint dicta, uel defuerint, tam in arte; quaim in dictionario ; cum facile fit addere; à doctiore fupplebuntur, qui tertiam artem, of feeurdum dictionarium aggredietur:ego enim ne d patre familias \& Domino Deo nostro reprebenderer, taientum nolui babere repofitum in fudario, fed duobus, alia fultim duo fuperlucrari, \& cooperari faluti Iaponiorum ; non foliem predicando, fed prabendo pradicatoribus, $\sqrt{2}$ po/fem infirisonenta lingue, o pracipuè modo ut po/fint illam extra Iaponiam. addifare: cum propter inflantem perfequutionem in I aponia uix fit boc poffibile. $V$ ale or uiue felix lector. Matriti die 30 Aug. Anin. 5631 . 
In hac arte Grammaticí ferua uimus ordinem, quemperitus Antonius Nebriffenfis, t) al ï feruarunt in fuis lingua Latine, per partes, videlicet Orationis, nempe Nomen, Pronomen, $(4)$.

\title{
De nomine \& cius declinatione, \& genere
}

\begin{abstract}
$N$ lingua Iaponica non funt declinationes per cafusficut in 1 Latina., fed funt quadam particula, quap poftpofita rominibus, cafuum; differentias conftituunt in omnibns nominibus, tam appellatiuis quaim proprijs. Qua confituunt nominatiüu, funt quinque, va, ga, càra, no, iơri. Particula, va , poftponitur quando quaji reduplicatiue, feu fpecificatiue volumus ex-plicare rem vel perfonam fignificatam per tale nomen; fiue fit prima, fiute fecunda, vel tertia perfona . v.g. Và tacùxi và mairàm, ego, vel, quod ad me attinet, non ibo. Particula, no, pofpenitur fecundis \& tertÿs perfonis, \& pracipuè fif fint inferioris ordinis; \& quando in oratione efl aliquod relatiutum, quod non denotat actionem tranfitiuam. v.g. Sònd́ta no mǒxita còto, id quod tu dixiffi. Poffponitur etiam particula, no, quardo aliquid indefinitum dicitur. v.g. iiè no ìrucà miió, videfifint domus. Particula, ga pofponitur regulariter primis \& tertÿs perfonis inferioribus, of etiam fecundis quando sunt etiam infima vel bumiliantur. v.g. Pedróga qita, Petrus venit. Solent etiä banc particulam postponere quando aliquid indefinite volunt dicere, ficut dictum ef departicula, no : v.g. còco ni và iiègd naica ? non funt bic domuss \& $\sigma f i$ in oratione fit relatiuum non dicens actionem tranftiuam, fir referat rem inferioris of bumilis ordinis, pofsponitur etiam particula, ga: v.g. Forégdxí ga càita fumi, Epitola, qnam egofcripfis, fochi ga iuta còto, quod tu dixifii. Particula, carra, \& iòri, poftponuntur \& faciunt nominatiuum quando oratio dicit actionem tranfitiuam, of precipue/s ff oratio relatiui . v.g. Deus iòri cùdafàrèta gracia gratia, quam Deus dedit. Tòno cdra còre vo vôxe tçuquerareta, Dominus boc pracepit. Aliquando ctiam nomina furit in cafu Nomina-
\end{abstract}


minatiuo ab qque aliqua particula. ข.g. Pedro Ioaó vo iòbarèta , Petrus uocauit Ioannem. Genitiuo funt due particule, videlicet no, or ga. Particula, no, feruit omnibus perfonis fuperio ris ordinis. v.g. Padre no vó qirumòno, Patris vesfis feu babibitus . ga, poftponitur omnibus perfonis inferioris ordinis . v.g. Pedró ga fumi, Epiftola Petri. fo chiga mòno, restua, àré ga càne, argentum illius, Tòno vàiòcú ga fucài fito già, Dominus eft magne cupiditatis, ideft valdc cupidus. Aliquando ctiam predictis particulis genitiuum conftituentibus poftponitur particula, to, v.g Pedronotodegozaru, eft Petri. Sednon eft modus loquendiperfectus. unde melius eft illo non uti ; ponitur tamen ut auditus intelligatur. Quando uerò coniunguntur duo nomina fubftantiua ad faciédum unum quaji connotatiuum, non eft ncceffaria particula genitiui . u.g. còcuxu, dominus regni. Secundum regulam tamen ordinariam deberemus dicere. còcu no xù, ¿ bic modus faciendi connotatiua ab fque particula genitiui, eft regularis in lingua Iaponica. u.g. Maria còto, res Maria.

Datiuum confituunt dus particula foiliset, ni or ie, u.g. Pedro ni móxita, dixi Petro. Fadre ie ague maraxita, Patri dedi, ue! obtuli.

Accufatiuum conftituunt quinque particule, vo, vóba, va, ie, ga, Prima uidelicet, vo, eftufitatior:u.g. Pedro voiòbe, uoca Petrum, va, utuntur quando uolunt in particulari explicare rem fignificatam per nomen in accufatiuo,u.g. niffion guchi vi Xiranu, linguam Iaponicam nefcio. Particula, vóba, eft idem quod, vo va, conuertendo fecundam literam, $u$, in, $b, e a$ uero utuntur ficus, va, u.g. fune vóba nòri futète; cane bacìri tòri mar d̀xità, relinquens nauigiturn : argentum feu pecunias tantum accepi; ie, utuntur ad fignificandum locum ad quem: u.g. Roma ie mairǒ, ibo Romam, g2, poftponitur nominibus jignificantibus res inanimatas, feu bumiles: u.g., àre ié gozdre, mònó ga móxitai, accedas illuc, babeo erim tibi aliquid dicere. fit etiam accufatiuum ab que aligua particula ut in exemplo antepenultimo in quo fecundum accufatiusm eft fine particula.

Vocatiutm conftituit particula, icani, fed non poftpolita nominibus ficut do reliqua, fed antepofita: $u$. g. icàni qimi còre vò gorònjerarei, uideas boc domine. Regularius uerö fit uocatiuum abfque aliquaparticula: u.g. Padre fàma qicàxerarèio, audias 
8 audias reuerende pater.

Ablatiuo funt tres particula, iori, càra, $\mathrm{ni}$, tertia foilicet ni, facit locum in quo: $v$ g. iglefia nig ozaru, ef in ecciefia: aliquando vituntur, ni, antepofito, no, v.g. fònàtd nò nì Xidru ca? facis boc tuum ? vel accipis tibi ? Sed bic magis videtur datiuum, quam ablatiuum. Particule cdra, of, iori, funt communiore sad ablatiuum conftituendum : v.g. Madrid càra màitta, ex Matrito veni, Pedro iòri coròfarèta, occifus fuit à Fetro.

Ad constituenda pluralia funt etiam quatuor particule, que poftpofite imme . atè rominibus, illa pluralia confituunt, pofiea vero, fequuntur particula pofite cafus conftituentes. Predicte autem quatuor particula funt, tàchi $\mathrm{xu}$, dòmo, $\mathrm{ra}$. Prime particula fcilicet, tachi, facit pluralia nomina fignificantia res nobilis ordinis, of quas volumus bonore afficere; v.g. Tòno tachi, domini. Particula, xu, constituit pluralia nomina fignificantia resnobiles quidem; fed non ita fupremi ordinis: v.g. Tamurài xu, nobiles; qui non funt domini. Particula, dòmo, pofponitur nominibus fignificantibus res bumiles, fue rationales fiue viuentes fenfibiles, five inanimatas: v. g. fildcuxó dòmo, agricole, ixi domo, petra, mmã dòmo, equi. Particzila, $\mathrm{ra}$, facit pluralia nomina fignificantia res vilifimas, vcl que deffeEiui babentur : $v . g$. ludeora, Iudai. Plutralibusfic iam ger ifias particulas conjiitutis, pofponuntur particule cafus conftitucntes fecundum orationum exigentiam: $v \cdot \dot{g}$. tòno tàchi no còtō dòmo vo varú iúnà, ne maledicas res dominorum .

Aliqua etiam funt vocabula, qua de je pluralitatem important: v.g. tòmó gàra, fignificat bomines, Nà bàn mòno, res Europeas, Nàn bàn mòno vo fomùru nà, ne laudes res: Erope:

Particula verò icàni, que, rt fupra dictum eft, facit uoca. tiuti non poftponitur pronominibus pluralibus iam factis; fed femper anteponitur, particula autem facientes plurale pofposuntur: u.g. icdni Padre tàchi vo qiqi nafarèio, audite reuerendi patres.

Dus uerò particula ex quatuor fuprapofitis, qua faciunt plurale, fcilicet domo, of rdaliquoties funt. fingulariù iuerbi gratia. vare rd, uel, midcomo, ego $:$ aliquasdo etiam utraque $f_{2}-$ mul inuenitur in fingulari: u.g. midomo ra, ego, midomo rã ga 


\section{DE NOMINE.}

mzeus vel mei : poftponuntur etiam predicte particule, domo, vel, ra, fingularibus, vel quando volumus bumiliare res fgrnificat as: aut de fucce/fu fignificati per orationem non fumus ita certi; fed quafi dubitamus: $v . g$ farä dòmó ga itài, dolet mibi venter, ¿̇fi dòrnó và àru mài, crastina die non erits àfu rà và naró mòce, crafina die forfatan ita erit.

Particula, va, polponitur nominibses tan fongularibus; quàrn fluralibus iam per particulas articulatis : $v$.g. còcolè va mairàru, bse non veniat, còco càra va dènu, bine non exiuit, còco ni và iru mài, buc non intrabit: aliquando etiam particula, va, fepplet particulas articulares: v.g. funè - de fàie ióió tçùita ni, cachi va nàcanaca nàru mài, finauigio vix peruent; pedibus, vel pedes, abfque dubio non perueniffem. Hic particula, va, fupplet particulam, cìra.

In ifla lingua I aponica non funt etiam genera, mafculinumvidelicet, femininum, or neutrum, ficut funt in Latina; funs tamen aliqua nomina, qua ex fe funt, vel masculina, vel feminina, quia ex fua fignificatione dicunt vel mafculum, vel feminam determinizté; funt etiă aliqua nomina ex fe cömunia duobus. Particula, vo, ante pofita nominibus fignificat masculum . Particula vero', me, antcpofita Jignificat feminam in rebus, que non babent genera propria : v. g. vo i vo, Jignificat pifcem mafrulum, mè ì vo, Jignificat pifcem feminam: vojica, Jignificat caprum filuefrem, mè lica, fignificat capream, còma, fignificat cquum; zoiàcu, equam; xô, , jgnificat accipitrem mafculum; dài, feminam, cotòi, Sggnificat taurum, meuxi, riaccam . votòco, fignificat virum, vonägo, vel uhoóbó, vel, vònna, $\sqrt{i}-$ ginificat malierem. In dictionario ommia expre/sè ponentur, qua ad memoriam vcnerint.

In nominibus etiam adiectiuis, non funt genera, aut declinationes; fed exdem particule illa conflituunt, que fubftantiua. Sunt autem adiectius mults, or diverfa. Aliqua enim finiuntur in, ai , alia in, oi , alia in, ei, alia in, vi, alia in, ij, alia verò, \& que proprius funt adiectiua, fiunt poftpofita particula, no, fubstantiuis. Quando quinque prima genera adieEtiuorum ante ponuntur Subflantiuis, tunc videntur proprie adiectiua , of nibil mutant ad orationem componendam; quando verò pofponuntur subflantiuis, potius funt verba, of verborum coniungationes fer quuntur : v. g. tacdi iàma, mons altus, xi guei ideiri, frequens 
Io

introitus or exitus, caxicòi fico, bomo prudens, cdvaij mòno, res mijerabilis, Aiaùi còto, rus periculo fa. Vmàre tçùqi nocùchi, lingua naturalis feu materna. Sunt etiam alya adiectiua finita.in na, que etiam ante po/ita.jubfantiuis, nibil mutant: v.g. qirèi na. còto, res munda. Omnia verò adiectiusa prater finita in, no, quando verbis praponuntur, mutant aliquid: finita $i_{i 2}$, ai , illud mutant in, ó, v.g. còno iàma và tacó gordru, bic mons altus eft, dr excelfus : finita in, ei, illud mutant in, ế, $v . g$. còno lama va xígueô gozàru, bic mons eft denfus . finita in, oi, illusd nutant in, ô, v g. caxicô gozdru, eft prudens. finita in, vi, illud mutantin, u, v.g. xei no ficú gozàru, Statura pufillus. finita in ,. ii, illud mutant in, iú, v.g. caiū gozaru, est pruriens, uel prurit, inter ifla uerò adiectiua finita in, ij, funt mul. ta qua ex uerbis procedunt: $u . g$. nozòmi, $u$, Jignificat defiderare :exillo ueró exit, nozòmaxij, quod Jignificat idem quod defiderabilis., e ; alia etiam procedunt.2x nominibus: $u$. g. varambe, fignificat puerum feu infantem : ex quo procedit vardmberaxij, quod figuificat ?d quod puerilis, $e:$ alia reperiuntur in dictionario.

Adiectiva werò finita in, na, quando anteponuntur uerbis, mutant, na, in, ni, u.g. fuxin ni zònzuru, dubium reputo uel pro dubio babeo.. Adiectiua uero finitain, no, conuertunt aliquando, no in, na , u. g. bèchino fito, conuertitur in, bechina fito, differens bomo : \& tunc fi uerbum fub fequatnr, mutat, na. in, ni, u.g. bechini goziru, eff differens. Senfus wero eftidem fiue finuatur in, no, fiue in na, $u$. g. bechi no fito no culhi càra qijta, eft idem quod bechina fito nocuichi cdra qijta, ex ore, uel ab ore diflincti uel differentis bominis audiui. Et tantium eft differentia; quod finitum in, no, nibil mutat, quid quid illi fubfequatur ; finitum ueri in, na, mutat, na, in, ni, ut dict um ef, fi uerbum fubfequitur. Si ad alia uero adiectiua fequatur uerbum fubftantiuum, oratio eft elegans : u.g. còno iama và tacó gozaru, bic mons eft altus ; fi uerò uerbum buiuf modi non. feguatur, eundem facit fensum, quia adiectiuum includit in $\sqrt{e}$ uerbum fubflantiuum; fed illo non utemur coram fuperioribus, non enim illis audientibus, 'dscemus, còno iamà va tacdi, fed dicemus, còno iamd và tacó gozdru. or fic in alÿs adiectiuis.

Adiectiua uerò finita in, $i$, aliquando; b fi rarò, illud conuertunt in, xi, nel qu, w. g. ioi , quod jignificat, bonus, a, um. fi- 
nitur in, iòqu, vel ioxi, v. g. iòqu, dancó xitè, faciendo bonam confultationem. Aliqua, of non pauca, funt nomina fubfrantiua, quibus, $\sqrt{2}$ poftponatur, na, fiunt adiectiua: v.g. afó, Agnificat inscitiam, ex quo deducitur, a fóna, quod fignificat id quod fatuus, a, um. Iiiu, $\sqrt{\text { Ignificat libertatem: \& }, ~ l i u n a, ~} \sqrt{\mathrm{g} g n i-}$ ficat id quod liber, a, um. que occurrerint alia in dictionario reperiuntur.

Aliqua funt nomina abfracta fubstantiua que antepofita vo. cabulis que ex fe fignificant bomines, fiunt adiectiua: v. g. lifi, fgnificat mifericordiam: fi uerò illi lin pofponatur, refultat, li ti jin, quod fignificat idem, quod mifericors, dis, fin, fignificat paupertatem, o po/pofito nin, fit, fin nin, quod fignificat pauperem . idem eft fipoftponatur, ja, fit enim, tinja, quod rtiam pauperem fignificat, bdn, sgmificat vigiliam; \&/s pofponatur, xu, fit, banxu, quod/sgnificat idem quod uigilans, tis. multa. inuenientur in dictionario.

Aliquando duo nomina fubflantiua, ex ÿs, que remanferunt in lingua I aponica ex Cbinenfi (do bac, cobita, vel, coie, vosant) fimul pofita, faciunt adiectiuum, quod ex duobus fubfrantiuis conflatur, feu refultat: v. g. ten, significat calum: \& , xu, fignificat dominum : tèn xu vero, fignificat dominum caleflem, feu celorum dominum.

Preterita etian verborum (de quibus fuo loco) adiunct a fubflantiuis, vim \& fenfum videntur babere adiectiuorum: v.g. iógorèta tè, manus fordida, iógorèta, est prateritum verbi iògore vru, quod fignificat idem quod forde 50, is, càita qió, liber fcriptus, caira, efi prateritum verbi cdqi , u, quod lignificas idem quod f cribo, is. Abftracta, feu radices ex quibus verba componuntur, funt nomina quaji verbalia fignificantia actionem in abfiracto: v. g. facdri, fignificat menfuram: ex quo refultat verbum, faciri, u, pro eo quo eft metior, ris, fajime, $\sqrt{\text { Ignifi- }}$ cat principium, fajime,uru, ef verbum, of fagnificat id quod incipio, is. Of fic in alys de quibus in dictionario. Antepofita particula, mòno, predictis abfractis feu nominibus verbalibus funt nomina fignificantia eum qui actionem verbi facit: v.g. ex , càqi, prepo fito, mono: fit mòno caqi, quod/Ignificat idem quod fcribens, tis . pofpofita vero cadem particula, mòno, cifdem radicibus, fiunt nomina fignificantia effectum aCtionis: v.g. cdigimono, Jignificat foripturam. 
12 D E NOM I N E

Polpofita verò particula, gòto, eifdem rudicibus verbalibus funnt nomina fignificantia res dignas actionibus que per verba, querum Junt.radices, Jgnificantur: v.g. mi, eft radix, ex qua prodit verbum, mi, iru. pro eo quod videa, es, \& , migoto, ef ves vijzbilis,feu digna vifit, qiqi , eft radix verbi, qiqí, u, pro eo

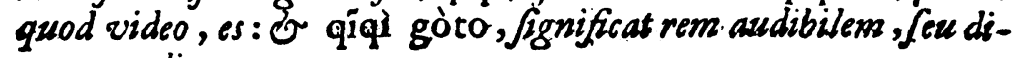
gnam auditu.

Poftpofitis aliquibus.nominibus fubszantius nominibus verbalibus prodictis fit nomen ians incancreta fignificans talem aEtiozem : v, g. foxi, eftradix verbi, fòxi, u. . proea quodfole vel ad folem ficcare: poftpofito vero', i vo, v.g. quod fignificat pifcem: fiet, toxii vo, quad Jgnificabit, pifces adjolem vel vifolis ficcos. bre.

Pofpofita vexò particula, dögษ, qux inftrumentü fignificat radicibus! verborum, funt nomina lignificantia materiam feu inftrumentum actionis per verbum fignificate : v.g. vardi dógu. wateria fexs inftrumientsm vifus, càqqi dógu, inftrumentum foribendivel ad foribendum.

Poftpofita autem particula, me, radicibus verbalibus funt nomina fignificantia terminum actionis: 8 . g. a vixe, eft radix. verbi, a vaxe, uru, pro.eo quod.eft aliqua iuxgere aut copulart, 2 vàxe me vero fignificat iuncturam: o fac in alys .

$E x$ adiectivis fuprapofitis finitis in i, fiunt nomina abftracta mutato,$i$, in , $f a, v . g$. nägdi , frgnificat id quod longus, $a$, um :

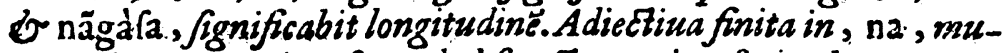
tant etiam, na, in, $\mathrm{fa}$, ad abftracta nomina facienda: v. g. ex dqiracdna, quod fignificat id quod clarus, a, um : fuet, àgiracàra, quod Jignificabit claritatem.

Aliquando ex duobus fubftantivis fanul fumptis, \& praciput faliquod illorum aliquam literam in principio feu in fine in alia mutet, refultat tertium nomen quafi connotatiuum, or quafi adiectiuum feu genitizuiu izcludens: v. g. ex, qi, quod frgnificat, lignum, or, fotoqe, quod. Jig.rificat idolum: fit, qi botòqe mutato , $f$, in , $\mathrm{p}$, guod fignificat idolum ligneum. Si verò nomira que proponustux, finiuntux in, $e$, ipfum mutant in 2, ad prevdictam compofitionem nominis connotatiui: v.g. tçumà fàqi , extremitates unguizum, càna cúgui, clauus ferreus. Si uerd nomina, qua pofponuntur ad eamdem formationem, primam. literam dobeant mutare , $\sqrt{2}$ fit,$f$, mutabunt in,$b$, wel, $P, \sqrt{i} ; s$, 


\section{DE PRONOMI NE}

mutabunt in, $\mathrm{z}, \sqrt{i} \mathrm{c}$, mutabunt in, $\mathrm{g}, \sqrt{2}, \mathrm{tc}$, mutabunt in, $\mathrm{zz}, \mathfrak{i}, \mathrm{x}$, mutabunt in, $j$, u.g. cdri bunè, bùppô, nígori zd. qe forá goto, qizzumári, fòra jèi mòn. Vide in dictionario.

\section{Depronomine.}

T On funt in lingua Iaponica pronomina deriuatiua u.g.m eus, I a, um.Ec. Sed utuntur primitiuis, fcilicet mei, tui, \&c. Hac autem primitiua non babent declinationes per cafus; fed ba differentia cafuum fiunt per particulas fupra pofitas que omnibus funt communes, tam nominibus; quim pronominibus .

Particzsle aliqua (d: quibus infra) qua adiuneta uocabulis banorem indicant, faciunt pronomen, uel illius uim babent fecussdum oscafionem or circumftantias in guibus illis utuntur: $\sqrt{2}$ enim ego dicam, von fümi ; loquendo cum alio: ip 5 facto intelligitur me de eius epi/fola: \& non de mea loqui : $\sqrt{2}$ enim de mea loquerer; :son dicerem, von fumi, fed, fumi tantum : unde, von fumi, raione particula, von, que eft bonoris, fignificat ueftram epiftolam. Et idem eft de particula, mi, of alys que bonorem indicant infynificatisnominum quibus adÿciuntur.

$$
\begin{gathered}
\text { De pronomine fecunde perfone fi- } \\
\text { licet ego } t \text { ) }
\end{gathered}
$$

Ctc funt particule frgnificantes idem quod Ego, nei, mibi, ¿cc. vátacuxi, fòrêgaxi, váre, mi, váre rá, mìdòmo, midòmo rá, váre. Quatuor prime denotant aliquam fuperioritatem in eo qui illis utitur, relique uero funt bumiliores. Mulieres utuntur tribus alys particulis aliquando qua funt. mizzu cára, väraúa vãgami, ơ bis non utustur uirt : ruftici folent uti duabus uidelicet, vára vòrará, religio fus utro quando de fe laquiture folet dicere, gufô, ac fi dictret : ego uilis religiofus ; fenex suerò de fe loquès: dicit, gurǒ', ego uilis \& defpectibilis fenex. Rex uerò , dicet a chin, wel, máru, quod fignificat : ego Rex.

$$
B \text { Ad }
$$


14 DE PRONOMINE

Ad facienda autem pluralia jita pronomina, poftponuntur illis particula Jupra pofita conflituentes pluralia, fcilizet, dòmo, ra, v. g. midòmó ga máitta tòqi, quanzdo nos iuimus : ad cafuum vero differentiä pofeponuntur illis iam formatis plur alibus, particule confituontes cafus ot fupra.

\section{De pronomine fecundx perfonę: fcilicet Tu,tui, tibi,\&c.}

$\mathbf{M}$

Vlta funt particule, qae constitunnt prononen fecunds perfone fecundum differentiam perfonarum, que vel nullum, vel aliqualem, aut mediocrem, magnum, aut maximum merentur boworem or reuerentiam: ad loquendum enim cum inferiori, funt tres particula que faciunt pronomen tu. Scilicet,

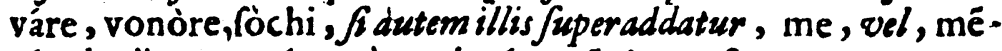
gá, ơ dicatur, váremè , vel, váremégá, perfonam cum qua, loquimur ampliius deÿcimus. Si autem loquamur cum aqualibus vel aliquantulum inferioribus, vtemur ana ex tribus particulis videlicet, fònaca, fóno fò, váre fama. Si verò jat perfona fuperior vel omnino equalis cum qua vrbane debernus loqui vtemur vna ex feptem particulis failicet, cònatá, qixò , qifó, gòfen, qíden, cònatá fama, fònata fáma. Si autem loquamur cum, perfonis in dignitatibus conßitutis,nomen dignitatis, fi illi fuper addatur particula, fama, supplet vicem pronominis: v. g. Padre famā gozare, veniat vestra paternitas. Aliquando etiam particula qua nomina bonorant, jupplent vicem pronominis: $v . g$. vòn qirumòno, vefra dominationis veftis.

Cònata, cóchi, cónofo, fignificant idem quod ego, mei, drc. fed in modo loquendi quafs diftributiuo: ex parte mea, vel quan-, tum ad me attinet, quibus correfpondent, fochi, fonofó , fónatá que fignificant, tu bre. or exparte tua, feu, quod ad te pertinet.

Pluralia ifforum pronominum fiunt per particulas fupra pofitas fecundum bonoris differentiam, vonóré domo, váre ra, fóchi ra, fignificant vos loquondo cum vilibus, vare tachi, fonatd domo, fignifica vos cum aqualibus, qî́ótachi, vócatãgata, vóno vóno, Jignificat vos cum bonore:declinationes vero.bo- 
sum foun etiam per particulas communes declinationum .

\section{De pronomine tertix perfon $x$, fcilicet ille, illa, illud.}

Va particula fcilicet, cáre cáre, are are, Jignificant ille, illa, illud, loquendo de rebus inferioribus: quatuor uerò uidelicet, áitçu, áitçumè , árcmè , cáutçume , fignificant ille, illa, illud, bumiliando b despiciendo res de quibus loquimur, of qua pracipus finiuntur in, me, funt de iectius, maxime fi illisfuperaddatur, ga, u. g. áitçüga, áitçumêgá , ille uílis: cóno, sgnificat bic, , bec, boc , sóno fignificat, ifte, ifta, istud, ano, /ignificat ille, illa, illud; fed necelJaria requirunt pof fe Jubftantiuum ut illis utamur: u.g. cóno fito, ifle bomo. idem fignificat, cóno mono, fed non eft uerbum bonorificum, sóno cóto, ifta res, ano fito, ille bomo, cónátá, uel cónofô, Jignifzcant bic, fónata, uel, fono fó, isthic, ánatá, uel, ánofó , illic. córe, Jugnificat boc, fóre, fignificat iflud, áre fignificat illud, fed neutraliter, ita quod fubftuntiuis, feu fuppofitis non iunguntur. borum pluralia funt, córerá, forerá, arevá, caterurum ucrò pluralia funt per regulas commones cáno, fignificat rem de quafacta ficit mentio: u.g. cano fito, ille bomo of c Pronomen quidam, facit particula, aru, u.g. aru fito, quidam bomo, aru tocóro ni, in quodam loco. fóre.

Pronomen unufquifque faciunt particula, mèn mèn, fóre

Pronomen uniuerfi \& finguli of. facit particula, tare mo $\min x$.

Pronomen quicumque \&o faciunt particula, Tare nite mó, tare nitcmoare, tare nari tómó.

Ante pofita particula, tare mo, megatiuis facit pronomen nemo uel zullus: u. g. tare mó mairananda, memo iuit. Particula nani taru cóto nari tómo, fignificat : quidquid fit, vel quecumque resfit. Porticula, mèi mès, fognificat : fingult, wel unufquifque in particulari.

Particula, góto, facit pronomen feu fignum diftributiuum omnis, e. Si pofponatur fubftantiuis ex proprijs uocabulis lin- 
guce I aponicie que uocantur, jomis . idem etiam facit particulan inai , antepo/ita Jubflantiuis lingua Cbina, qua uocantur, coies, ajg. fi, fignificat diem: $\delta$, figótoni, fignificat quotidie, feu omnibus diebus, nen, in lingua laponica mendicata à Cbinenfis, fignificat annum: $\mathcal{O}$, main nen, fignificabit ingulis annis uet omnibus annis ;idem fere facit reduplicatio aliquorum nominum, uel Saltim facit illa pluralia: $u . g$. fito, /gnificat bominem : or, fitöbito, fignifucabit onnes bomines, wel mult os bomines, \$1, fignificat diem: or fibi ni, fignificabit, omnibus, wel multis diebus.

Pronomen diuifizum, aliqui orc. facit particula, niióte, u. $g$. róqi niiotte, aliquibus temporibus, fito niiotte, aliqui bomines.

Pronomen, idem Juc. facit particula, ronaji, u.g. vonaji rocóro cara, Ex endem loco. Particula uerò, dôjen, Jignificat idem, fed neutraliter : v. g., dôjen degozàru, idern eft: ऊ boc uerbo refpondent quando gratulantur; abfque eo quod rem accipiant: ac fi dicant, gratiulor ac fi accepilfem : uelidem eft, ac $\beta$ accepiffem.

Pronomen ipfe Jc: faciunt particule, nùxi, fóno mi, vãga, particule uero, vareto mi, faciznt pronomen ipfemet Jcc.u.g: vareto mi ni ata vo nafu, ip/emet fibimet damnum infert, mi vo valnrète; ta vo rafùqùru, fui met oblitus, alios faluos ' facit. Particula, vatactixi , fignificat rem propriam in particulari : $u$. g. vatacuxi no còto, res propria, vatacùxi ni iuaréta, proje. logusutus eft.

Pronomen aliquis Üc. facion tparticule, Tare zo, talo, $u$.

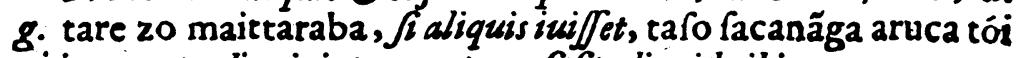
ni iqe, eat aliguis interrogatuon $\sqrt{2}$ fit aliquid cibi.

Aliquid neutraliter faciunt particula, nan zo, nanica, u. g. nanzóga aräba cùuózu, comedan aliquid/ $/ 2$ fit, ima faia tê ga jiiu ní gozaru fódoni nanica caqi maraxô, frribarm aliquid, $\sqrt{b}$ quidem babeo manus iarn folutas fou liberas .

Quis ? fit tribus particulis foilicet, Tare, Tăga Tarc, panticule tăga, uel, tare nó, utusutus etian pro genitiuo cuius : u. g. Täga móno ca ? cuius eft res? Et quando aliquis ad oftium wocat, on pulfat dicit: móno mó, qur intus refpondent, tafo, uel, tagá, uel, tare,quis es 3 Particula nani, fignificat quid óc. u.g. nani 
ro füru cá, uel, nanigoto volùru ca ? quid faris? nani ni fóre vo tòtte iquca? ad quid bor portas tecum ?

\section{De pronominibus rela- tiuis.}

Donomen relatiuum fit poftponëdo verbo nomen de quo fit re1 latio: v. g. tèn ni mìximàlu vàrerága vòn vòia, $P$ ater no. Ater qui eft in calis, dèta tocòro va, locus ex quo exiuit, tèni fùmi no tçuita fito, bomo cuius manui adbefit atramentum Si oratio petit nominatiusum ante verbum, tale nominatiuum debet effe cum aliqua ex particulis nominatiui, ga, no, uel, iòri, v.g. vatacùxíga càita fümi, epiftola, quam ego $\int \mathrm{crip} f \hat{i}$, cònàtà no vôxerarèta coto, res quam uefira dominatio dixit. Tertia. verò particula fcilicet, iori, vtimur quando eft aEtio in oratione,q. g. Deus iòri atàie cudafarèta gracia, gratia, quam Deus. contulit feu donauit, àno tocòro ni amàta no qió d̀tta vò torarèta, tulit multos libros, qui erant in illo loco. Si concurrant verò duse orationes relatiuum continentes, prima erit fecundum pofitam regulam; fecunda verò per participium prafens, prateritum, feus futurum, fecudum quod fenfus orationis poftulauerit: -.g. qèra Oracio vo móxita qióga tçucuie no vie ni àru vo motte coi, affor librum illum qui est fuprafedile, in quo ijto mane recitaui vel dixi officium diuinum. In ifta oratione qióga, quod eft unum relatiuum, eft poft verbum, móxita, \& vo, quod agit vices fecundi relatiui, ef post verbum, dru . Quando verò volzumus amplius specificare rem que refertur, ponimus inter rem ip fam \& verbum, particulam tocòro no, v. g. vdreto dôxin xìta tocòro no mònó dòmo va minabuguèn ni nàtta, omnes quotquot mecum confenferunt, diuites facti funt. Aliquando oratio relatiui propter fuam difficultatem explicatur per exponentes: v. g. loco buius, ima còrofarèta Pedro nò cò và fonàta nó chijn gia, que /gnnificat, filius Petri, qui modo fuit occifus, eft tuus amicus: dicimus, ima Pedro corofarèta fonó co va fonatà no chijn de gozàru.

Aliquando folent fimul poni dua particula ex cafus conftitustibus, \& faciunt quajis relatiuum cui anteponuntur: v. g. fòno 
tocòró deno dancó, iffius locis confultatio. Marfella ieno fúne, nauigium quod tendit Marfiliam.mdire to no móxi gòtó dearu eft dicere, quòd eam.

Màiru mai to no dancó ni qivamatta, babita eft refolutio quod non pergat, màitte nòchi no dancó, confultatio poftea quamperrexit facta, varambe càra no catägui, est confuetudo ab infantia, xô tameno chôgui gia, ef ars ad illudfacienduon : dno fito no vo toró, accipiam id, quod est illius bominis. nota bac de relativo valde.

\section{De formatione verborum, \& conirt- gationibus .}

$\mathrm{V}$

Erba in lingua Iaponica neque babent numeros, neque, perfonas; faciunt tarien bas differentias particula fuprapofite ad pluralia of declinationes. Coniugationes funt tres affirmatiuse, of totidem negatiue.

Radices verborum, de fe non dicunt tempus: vonde ot illud disant debent formari verba \& coniugari.

Omnes radices verborum fecunde conirgationis finiuntur in, e, gi, vel, $j \mathrm{i}$, prater, $x i, \&$, maraxi, que et fi finiătur in, $i$, funt tamen prime coniugationis. Siverò radices finizentur in, de, ve!, gi , fit verbum prafentis temporis conuertendo predictas in, zzuru, v. g. fägi , facit prafens, făzzuru, \& fignificat erubefco: de zzuru, quod Jgnificat exeo, is . Fi radices finiuntur in, je, vel, ji, mutantur ad prefins in, zuru, v.g. màje: mãzuru, idem quod mifceo, es, anji anzuru, quod eft confidero, as, fifiniuntur in, xe, illud mutant in, furu, v. g. 'a vaxe aualùru, idem quod coniungo, is : xi, vero, of maraxi, que (vt disfum eSt) Junt fecunda coniugationis, etiam mutant, $\mathrm{xi}$, in furu, v. g. xi furu, idem quodfacio, is, maraxi marafuru, etiam$\mathscr{f}$, facio facis. Si radices finiuntur in, te, conuertunt illud in tçuru, v. g. fodì̀e ; fodàtçùru, idem quod alo, is, vel fuflernto, as: reliqua qua tantum finiuntur in, e, alio modo; illud ad prafens conuertunt in, uru, v. g. ãgue : âgiru, offero, rs, nigue, niguru, fugio, is .

Aliquafunt praterita verborum, qua faciunt fenfum prafen- 


\section{DECONIVG}

tis, b. funt illa quorum fieri confflit in facto effe: 0.8 . cocòroièta intelligo, is, qicoieta, audio, is, voboièta, recordor, aris, qi qi ièta, intelligo, is, zonitia, fcio, is, os alia preter ifa forfan crunt. verba qua fequïtur funt prima coingationis etiam fi eorum radices non finiantur modo antea dicto. Si prafens alicuius ex illis non fuerit mutato, $i$, in uru, explicabitur in particulari, àbi, uru, aque balneofe, abluo, is, fotòbi, uru, molifficor, aris: focoròbi, uru, difjuor, eris:cibi, fucore afficior, eris, fabi, rubiginor, aris, deqi, firior, ris, velperficior, ris: cuichi, cutcuru, putrefso, is : michi, mitçuru, mare adimpleor, ris, ini, uru, abso, is : nòbi, nobiru, vel noburu, dilator, aris, tçuqi, uru , confumor, eris, vòri, uru, ab alto defcendo, is: xij, xijru, ad prandium vel cibum compellendo, inuito, as, ni niru, affimilor, aris : mochii : mochi iuru, afimo, as:ni, niru, ad ignem coquo, is: mi,miru, appicio, is : cori , uru, corrigor, eris, vochi vôtçuru, cado, is : $i$, iru fum,es, fui : vel adfum, es, tügui, uru, tranfeo, is, ficut tempus tranfit: vabi, uru, mifericordiam peto, is, carabi, uru, ficcor, aris, iqi iqùru, viuo,is, fi firu are $f c 0$, is, qi qùru, venio, is, qi qùru, vefio, is, vòqi uru, e lecto furgo. quatuor verò verba qua fequütur babent prater prafentia ordinaria, alia etiam extraordinaria,. atàie, babet atoru, pro dono, as : vdquimaie, vaqimòru, difcerno, is, tonàre tonóru, berredico, is, fonaie fonóru in loca fublimi colloco, as.

\section{DePręterito imperfecto, perfecto, \& pluf- quam perfecto.}

T On efi in lingua ifta Iaponica preteritum imperfectum: V Vide loco illius vtuntur perfecto, quod fit duobus modis: primü eft addendo, ta, radicibus verborum: v.g. äguèta, ef prateritum verbi, ägue, uru, quod eft offero, rs; fecundo modo fit prateritum perfectum poßponendo, te, radicibus, o addendo postea verbum, gozari, $u$, vel ari, $u$, in prafenti vel in praterito de quo in Jecunda coniugatione, v.g. äguete gozdru, vel aguèté gozd̀tta, vel ãguete aru, vel äguete atta, obtuli, wel obtulerem, as, \&c. Si autem ad pradicza anteponatur particu- 


\section{Imperatiuum primx coniuga- tionis .}

$I$ Mperatiuum prime coniugationis ef ipsa fola radix verbi, vel polfpofita particula io, v.g. âgue, vel ägueio, offer: futurum autem imperatiui elf futurum abfolutum, ãgueô, vel ägueôzu, É ef honoratior modus loquendi è vrbanior ad imperandum in omni coniagatione, quam per imperatiurem abfolutum. Fit etiam imperatiuum, $\sqrt{f}$ auferas, nu, a prafenti negatiuo (de quo postea) \& loco eius pionas fai, v. g. Siab âgue nu, tollas nu, $\checkmark$ addas, fai, fit ägue faj, quod eft, offer : non tamen dicit tantum imperium ficut abfolutum. Pa/posfita etiam particula, tai, radicibus, fit quodä genus futuri feu optatiui quo defiderium laquentis explicatur, is est imperatiuum ; vrbanum tamen, miz$z \mathfrak{u}$ fitòtçu nomi tai, vellem parum aque bibere: eft idem quod da mibi bibere. 2uando verà fit relatio alicuius precepti, legis, corsilij, ordinis, aut probibitionis, in omni verbo cuizufcumque coniugationis sue affirmatiuc, fiue negatiua: tale preceptum ponitur ad literam: v. g. chriftiani naru nd to no xŏgun no fattóga dru, eft lex Imperatoris, quod non fat quis Cbriflianus, Padre core vo còxiraièio to voxerarèta niiòtte, quia pater pracipit mibi vt boc componerem.

\section{Optatiuum primx coniuga- tionis.}

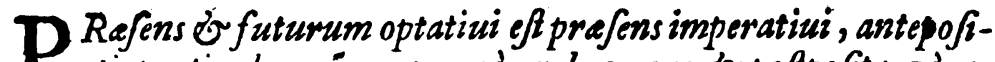
P tisparticulis, négauacu ud, vel, a uare \&o pofpofita, gàna, vel caxi, fit etiam po/ppofita particula, gdna, abfque aliqua antepofita: v.g nëgavdcu va ägueio càxi ? vel audre aguei gàna, /z offeres? auàre icanaru tèngu, bangue mòno nari tomò, vare vo tàtte, fiie no iama ni noboxèio caxi! ofieffet aliquis vel diabolus v. $l$ arufpex qui me afcendere faceret ad montem qui vocatur, fie ! po/ppofita particula gana, nominibus, $\sqrt{i-}$ gnificatur defiderium rei fignificato per nomen: v.g faqe gana, 
ò vinum! ac fi diceret : quis illud baberet ad bibendum! nani gana! fi aliquid baberem!

Prateritu:n optatiui eff fecunda wox futuri pofpofita particula mono vo! v.g. nigueo zu mono vo! o fi fugiflem ! idem fit boc modo níguéta raba iocaró mòno vo, aliquando folum dicunt, níguè taróni va letiam dicunt, niguete aró ni ua iocarómòno vo!

\section{Subiunctiuum primæ coniugationis affirmatiux .}

D Rafens Jubiunctiui fit ex prefenti indicatiui mutato, $u$, in 1 quo finitur in éba, v.g. ex, ãguru, fit ãguréba cumofferem: fit etiam ex prafenti addita particula, tocoro, fuper addite $\mathrm{ni}$, de, uo, vel, wa, fecundum exigentiam declinationis cerbi quod fequitur; primum enim fubit munus nominis: $v . g$. arutogi Pedro chinfui xitè iraruru tocoro ie fitó gaqite, cum veniffet quidä bomo ad locum vbi erat Petrus quando erat ebrius nhóbó ni rachi vacarète iru tocòro ni, cum elfent diuifi, $\mathcal{G} d i$ uortium feciffent coniugati, có aru tòcorô de, cumbac ita. fint, iòlo ie zzuru tocòroù fito ni corofarèta, occifus efta, quodam bornine cum exiret foras, go mifa vo afobafarùru tocò ro vo uchi coroita,occidit illu cum actualiter mifam celebraret, of eft regulageneralis in omni coniugatione.

Frateritum perfectum or plufquam perfectum fubiunctiui fit ex praterito perfecto indicatiuipo/tpofita particula, rëba, $v . g$. ãguèta réba, cum obtulifet: fit eticim ablato verbo gozaru, à preterito plufquam perficto; o pofito loco eius, attaréba, vel atta, quando vero ponitur, atta, debet fuperaddi vel, ni, aut, uo, ua, vel, 1e, fecundum quòd petit Jub equens verbum ; ad modum fupra pofitum de prafenti fubiunctiui cum particula, rocòro, v. g. ãguète atta réba, vel ãguete atta, ni, no, ua, vel, ie, cum iam obtuliffet.

Futurum /ubiunctiui fit addendo futuro indicatiui particulăs, tòqi , v.g. ̃̂̉ gueô tòqi , cum poftea offerat.

Prateritum plufquam perfectum fubiunctiui, vel quomodocumque illud voces, denique ad jignificandum boc quod est, poß- 
quam fecifem actionem verbi, fit poftpofitis particulis, cara, nòchi, vel igo:preterito plufquam perfecto; ablato tawese verbo, gozaru, v.g. aguète cara, nohi, vel, igo, mairó, poftquanz obtulerit profici icar. idem qua $\sqrt{2}$ ef , aguètaró toqi mauró, proficiscar quando iam obtulerit, ägueôzurù ni, vel, águeozuru tocoroni, /gnificat, cum iam eflet paratus ad offerendum: vel wt offerret, ãgueôzuru còto no fagini, fignificat paululum antequam offeret.

Prefens permiffurum Subiunciui fit duobus modis: primus $\varrho$ conuertendo, $\mathrm{v}$, in quo finitur pra jens indicatiui, in, ëdomo, v. g. ãgurédómò etiam fi offerat.

Prateritum verò permi fiui fit po/pofito, redomo, preterito indicatiui: v.g. äguetarêdomo, quamuis obtuliffet: futurum autem permiffiuum ef addendo, redomo fecunda voc $i$ futuri indicatiui: v.g. ägueôzurēdomo, quamuis offerat. Secundus modus fubizinetiuipermifiui eft efficacior of fit prafens pofpofita particula, tomo, prefenti Indicatiui: v.g. ãguru tomò quăa uis offerat: Solent etiam prafenti poltponi particula, mamaiò a vel, madeiò, v.g. forcuo voxiiùru mamaio, vel, fòre vo voxiitiru mãdeio, etiam fi boc doceat .

Proteritum autem buius fecundi permiffui fit pofpofito, rìtomò , praterito indicatiui : v. g. ägueta ritomo, quamuis obेtulerit: fit etiam boc proteritum poftpofito, mamaiò vel madeio, preterito indicatiui : u.g. ãgueta mamaio, uel, ägueta madein idem fit pofzpofito, tòte, praterito fubizinctiudi: u.g. ãguctarêba tòte.

Futurum permiffiuum fit pofpofito, tomo, fccunda uoci futurizindicatiui: ut.g. águcozutomo, fit etiam po/pofitis, mamaio, uel madeio eidem futuro: fi uerò pradicto permifjuo in. omnibus temporibus anteponatur, tatòi, additur magna uis orationi : u.g. tatoi vôxerarùru tomo, quamuis boc pracipia s.eumdem fenfism facit prateritum plufquam perfectum Indicatius ablato zuerbo, gozaru, \& , aru, \& conflituendo loco eius particulam, mo, u.g. ãguete mo, quamuis offerat . eadem particula, mo, poltpofita prafenti indicatiui facit eumdem fenfum: $u . g$. dòcó de giqi marafùru mo, fòno fata va mólanu, quarmsis axdiatur de boc ubicumque; nibil tale auditur: eumdem fenfum Solent facere modi loquendi, qui fequütur, ã guemo xèio caxi? ãguetemo xô madeio . nanto mo ägue caxi?quod fere /zgnificat , 


\section{$24 D E C O N I V G$}

quamuis offerat. exundem etiam fenfum faciunt prodicta, ãguruni faxerarei âgu etani faxerarei, uel ãgueò ni laxerarei, etiams fiofferat, obtulerit, uel etian fi offeret : ac $\sqrt{i}$ dicat : ponamas, wel domus quòd ita fat.

\section{Infinitiuum .}

D Rafers infinitiui fit ex prafenti indicatiui, poftpofito còto, $u e l$, to ,u.g. ãgùru còto, tzel ãguruto, offerre.

Prateritum infinitiui fut poftpofitis eifdem particulis praterito indicatiui,u. g. ãgueta còto, vel, ãguetato,obtulifle. Futurum infinitiui fit eifdem poftpofitis futuro indicatiui: u.g. ägueeô còto, uel ãgueôto, oblaturum. Eundem fenfum faciunt prefens, prateritum, \& futurum indicatiui poftpofita illis particula, ióni, u.g. nai nai guioi ni caqerareô ióni va vare mo zònzuru fitó bìo mo zonjita, fape credidi fo aly etiam putarunt me d te beneficÿs effe afficiendum, qèccu vare ni voxiie marafüru ióni gozaru, potius ille poteft me docere, ãgueta ióni gozaru, dicitur illum obtuliffe.

Ad interrogandum of refpondendum utuntur Sepifime infinitiuo quod eft fuppofitum uerbi quod fubfequitur : u.g. nhobógata ni vòchita cotó gaatta ca 3 incidisti ne in pecciatum luxurie cum muliere? fuit ne boc quod eft incidife orc. So boc modo loquendi utuntur in omni tempore infinitiui.

Aliquando Jupplet infinitiuum preteritum plusquam perfectut ablato uerbo, gozaru, \& aru . u.g. Deus no minòri vo firomète iòcaró, bonum eft legem Deipropagari: aliquando fupplet prafens o prateritum infinitiui, prafens \& prateritum indicatiui poftpofita, ga, u.g. fore vo vôxeraruru ga varú gozaró, malum erit boc dicere, maittágamaxi gia, ueniffe eft melius, aut effet melius.

2uando uerò infinitiuo fubfequitur uerbum fubftantiuum, non indiget particula, còto, u.g. còfacazzuqí dè và fàqe vo nòmu deuanai, bibere uinum calice paruo non eft bibere, còre còlo caqu degozare, boc polfumus dicere effe uerè fcribere, caqu degozate còfo, boc nullo modo eft fcribere, fòre ua ãgùru deuanai, iftud non eft offerre: aliqua ex exemplis pofitis funt ex uerbis alia- 
DE C O N I V C.

25

rum coniugationum; fed regula eft generalis in omnibus: facit etiam fenfum quafi infinitiui modus bic loquendi, águe va, agure domó, quamuis offeram vel et if faciam boc, quod eft offerre : eft etiarn regula generalis in onnious coniugationibus: unde dicunt, qìi va tçucamatçure dòmò gàtten xenu, quamuis audiam uel faciam boc quod eft audine ; xon intelligo . dicitur etiam, ãgùru vomotte, offerendo, wel cum boc quod eft offerre, ãgùru iori, ex boc quod eft offerre, ãguru nitçuite, cirea boc quod eft offerres.

Gerundium in, Di, es presens uel futurum indicatiui, of precipue $\sqrt{i}$ adiungatur, jibin, aut aliqua particula fignificans tempus:u.g. aguru jibùn, tempus offerendi, ãgueô ni qiuamàtta, accopit refolutionem offerendi, niguru jibun gia, temspus eft fugiendi, coròla réôzúru ni aifadamatte arozu,erit refolutus occifu, aut quod erit occidendus .

Gerundiü in, Do, fit duobus modis. primus, pofiponendo, prefenti indicatiui particulas, ni, uel, tòte, $u . g$. aguru ni , zuel, águrutore iurnfareta, offerendo fui folatus. Secundus eft auferendo uerbum, gozdru, praterito plufquam perfecto: u.g. aguète cutabireta, offerendo, vel erigendo fum defe $\int$ us : ideft ex orectione uel actione offerendi, refultauit effe defeffum: eft etiam alius modus elegans gerundiy in do, \& ualde communis, anteponendo radices uerborum aly suerbis compofitis: u.g. fiqi iolüru, approximare trabendo; radices autem $\sqrt{21}$ uerbis adiuncta num qu mutätur in pa/fizsis, neutris, aut negatiuis.In ifto Jenfu gerundÿ in do, uidentur vti bis modis loquendi taixó to xite, cum effet dux : uel ducis munus gerendo, vón rei to xitè gratias agendo, ròtai nòmi ni xitè, cum fit fenex, tçùcài xite iuaruru, dicit ut nuncius.

Gerundium in dum, fut poftpofitis particulis, tàme, vel, tòte, prafenti vel futuro indicatiui : v. g. ägùru tàme, vel, ãguco tòte ad offerendum, ad eundem fenfum redusitur bic modus loquédi , águru ni fàttóga àru, ost lex circa offerendum, nifí dicamus boc vltimum effe gerundium in ni.

Supinum in Tum, fot duobus modis, primus eft postponendo ni, radicibus: fecundus postpofito, tameni, prefenti indicatiui: v. g. tàzzunè ni maitta, vel, tázzunùru tameni màittá, veni oblatum.

Supinum in $T u$, est fola radix verbi. in boc etiam fenfu vi- 
dentur vti iflo modo loquendi, mófu ni vòiobànu, non eft necesfarizem dictu .

Participia prefentis, prateriti, of futuri funt prafens prateritum, \& futurum pofpofitis particulis, fito, vel, mono, fed quasdo poftponitur, fito, eft modus loquendi bonoration: v.g. ägùru fito, vel, ãgùru mòno, offer $\breve{s}$, ägueta fito, qui abtulit, agueô mòno, qui offeret, Buppô gacu furu tòmógara ni voite ua, vacantes fudia legis idolorum, von vo xiru vo fito to va iüzo; von vo xiranu vóba chicuxó to còlo iie, in ifta oratione particula, uo , fupplet vocem participy of dat fuppofitum verbo fignificatque: meritò vocant bomines beneficium cognofcentes; ignorantes verò beneficia iure vocant belluas. eft regula generalis in ornni coniugatione, vnde exemplum eft in verbo fecunds coniugationis. fit etiam participium postpofita te, radicibus verborum: $v . g$. aguete, offerens.

\section{Prima coniugatio negatiua.}

$R$ Adices funt postpofita, zu, radicibus affirmatiuis: $v . g$ Prafens verò efl conflituendo, nu, loco, zu, v. g. äguènu, non offero, of eft regula generalis quornodocumque finiastur eorum radices, fola, xi, of, maraxi, faciunt prafentia negatiua, xènu maraxenu, non facio, ea verò quorum radices finiuntur in $j i$, mutant $\mathrm{ji}$, in je, î postponitur illis particula, nu, adpresfens : v.g. zonji , fit prafens negatiuum, zoniènu, nefcio. alicubi in Iaponia faciunt negatiua auferenda vltimum, v, à radicibus negatiuis \& fuperaddendo illis verbum, ari, $\mathrm{u}$, coniugatum per fecundam fecundum tempus : v.g. ãguezàru, non offero, ãgue zàtta, non obtuli, ãgue zatta réba, cum non obtuliffet, etiam dicunt, ãguezu xite, non offerendo.

Preteritum negatiuum fit ad modurs prefentis ponenda loco, nu, particulam nanda, v. g. äguenànda, non obtuli, zonjenan$\mathrm{da}$, nefciui, vori nànda, non defícndi.

Preteritum plufquam perfectum fit mutando vltimam a, prateriti in, e, o postponendo verbum, gozdru, in prafenti, vel, gozdtta, in praterito: v.g. äguenandê gozaru, vel ãguenànde gozàt- 
gozàtta, non obtuleram. fit etiam ponendo, idé gozàru, vel, idê gozatta, loco nandé gozaru, v.g. ãguèi de gozaru, vel, ãgueidē gozdtta, non obtuleram, zonzeidể gozaru, nefciueram, vochiídë gozatta, non cecideram .

Futurum negatiuum efl addendo, mài, vel, màji , radicibus, vel prefentibus affirmatiuis: : v. g. ägue mai, vel ăguru maji, non offeres.

Imperatiuum fit po/spofito, na, prefenti indicatiui : v'g. ãgùruna, ne offeras.

Fit etiam antepofito, na, \& poftpofito, fo, radicibus affirmatiuis: $v . g$. na ägue fo, ne offeras.

Fit etiam postopofito, na, radicibus: $v . g$. ăgue na, ne offeras, mixè na, ne ofendas, mefare na, ne faciatis . radices, qua $f-$ niuntur in, xi, vel, $\mathrm{ji}, \& \int$ unt fecunda coniugationis mutant illud in, $\mathrm{e}$, ad istud imperatiuum: v. g. To xè na, vel fó maraxe na , ne facias istud, fo zonzena, me ifiud cogites.

Optatiuum fit anteponendo, negauacùua, vel auare, \& pofponendo, caxi, gana, imperatiuo negatiuo: v.g. auare aguru nacaxi, ofi non offerres: vel, négauacu ua na àgue fó gana, idem.

Preteritum optatiui fit pofpofito, mòno vò , futuro negatiuo: v. g. äguru mai mono vo! o i non obtuliffet!

Subiunctiuum verò negatizum est conuertendo, $y$, in qua finitur prefens negatiuum in éba, v.g. äguenéba , cum non offerret.

Preteritum fubiunctiui eff postpofito, rẻba, praterito negatiuo indicatiui : $v$.g. ãguenanda réba, cum non obtulifet .

Futurum eft pofppofito, qerëba, futuro negatiuo: v. g. niguru mai qerēba, cum non fit fugiturus.

Subiunctiuum permiffuum fit poftpofita particula, dòmò , prefenti negatiuo, Sed mutato u, in quo finitur in, $e, v . g$. aguené dòmò, quamuis non offerat . dicunt etiam or melizus àguenaidemo, vel, ãgueídemo.

Prateritum permiffiuum eft pofpofito, redomo, praterito negatiuo: v.g. äguenanda rédomò, quamuis non obtulerat, dicunt etiam, äguenaidemo, vel, agueidemo, \& finon obtulerit.

Futurum permiffurum est poßpofito, qerédòmò, futuro negatiuo: v.g. ãguru mai qerédòmò , ¿े fi non offeret.

$D_{2}$ Alitud 
Aliud autem permi]fiuum cum particula, tomo, fit pofpofitiz predicza particula radicibus negatiuis: $v . g$. äguèzutomò, non offert. Fit etiampoltpolito, totc, prefenti fubiunctiui: v. $g$. ãguenéba tote, tertio modo etiam fit po/pofito, mamaio, vel, madeiò, prefenti negatiuo: v. g. ãguènu mamaio, vel, âguenu madeio, quamuis non offerat.

Prateritum fit poflpofito, ritomo, praterito negatiuo: v.g. åguenandari tomò, $\delta \sqrt{2}$ non obtulerit. Fit etiam pofpofits, tote, praterito negatiuo fubiunctiui : v. g. aguenanda rêba tote or melius, ãguenaidemo, vel, ãguèidemo, quamuis non offerat, vel obtulerit.

Futurum eft pofpofito, tomò, futuro negatiuo: v.g. ëgue

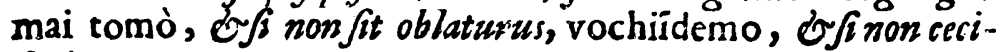
derit.

Infinitiuum prafens, prateritum, \& futurum, ef ipfumprefens, prateritum, of futurum negatiuum indicatiui, pofpofitis còto, vel, to , v.g. ãutrenu còto, non offerre, äguenanda còto non obtuliffe, ãguru mai còto, non effe oblaturum.

Aliquando vtuntur presenti negatius pro preterito in ommibus coniugationibus: v.g. mi maraxènu, non vidi.

Gerundium in, Di, negatiuum, effreferzs vel futurum negutiusum, águenu, vel, ãguru mai, non offerendi.

Gerundium in, Do, fit po/pofito, ni, radicibus negatiuis rel prefentibus: v.g. ãguizuni, vel, äguenuni, non offerendo, idem quaji eft: âgueide, vel, ãguenaíde, vel, ãguèzu xitè.

Gerundium in, Dum, eftpo/tpofito, tote, vel tame, prefenti aut futuro negatiuo indicatiui: v.g. ãguenu tame, vth, ágùru mai tòte, ad non offerendum.

Participia negatiua prafentis, prateriti or fituri, funt praens proieritum, or futurum negatiuum postpolito, fico, vel, mào v.g. ãguenu fito, non offerens, ãguenanda mòno, qui non, obtulit, äguru mai mòno, qui non offeret, äguena íde cara, vel, ãgucide nòchi, poft non obtuiffe: vel poftquam non obtulerunt, aut poftquam non efl oblatum. 


\section{Secunda coniugatio affirmatiua .}

$\mathrm{O}$ Mnes radices verborum fecunda coniugationis finiuntur in, i, fitque prefens mutato, i, in, u, v.g. iòmi : iomu,lego. Si radices finiuntur in, chi, mutant iftam dictionem in, tçu v. g. machi : matçu, expecto. Si finiuntur in, xi : mutatur in fu : v.g. coròxi : corolu, occido.

Preteritum fit. Si radiaes finiwntur in, ami, conuertitur in, óda,v.g. cami : códa, mandi velmafticaui. Si finiuntur in, ebi, vel, emi, mutantur in, ê̂da, v.g. faqèbi : faqeôda, vociferatus fum, fonèmi, loneoda, inwidi feu babui inuidiam. Si finiuntur in, òbi, vel, òmi, conuertuntus ì , ôda, v.g. coròbi : corôda, cecidit: còmi, côda, Je inclufit. Si finiuntur in, umi, conuertitur in, unda, v.g. cafùmi : cafunda, obtinebriitss eft, in idem conuertuntur que finiuntur in, imi, v.g. canaximi, canaxúnda, triflatus ejt. Si frniuntur in, gui, illud conuertunt in, ida, v.g. fëgui, feida, difcifum est. xini, uru, facit prateritum, xinda, mortuius $e f: \mathcal{O}^{\sim}$, ini, uru, facit preteritum, inda, abiuit: \& quantum ad boc funt ficut fecusndce coniugationis; quo ad alia vero tempora funt prime. Radices que finiuntur in, chi, vel, ri, illud conuertunt ad pratcritum in, tta, v.g. mòchi : mòtçu, facit prateritum, mòtta, accepit, chiri, u: chitta, fparfum ef, que veró finiuntur in, xi, vel, qi, illud couertunt in, ita, v. g. coròxi, u : coròita, occidit, qiqi, $u$, qijta , audiuit, xiqi, u, xiita, extendit.

Futurum fit conuertendo, $\mathrm{i}$, in quo radices finiuntur in, ó, ozu , vel ózuru, v.g. iomó, iomozu, vel, iomózuru, leges. $f i$ qerò radices finiuntur in, chi, mutatur ifla dictio in, tó, $v . g$ machi : mató, expectabo, qua finiuntur autem in, xi, illud conuertunt in, fó,v.g. móxi: u : mófó, dicam, aut loquar.

Imperatiuum fit coruerterdo, $\mathrm{i}$, in quo radices finiuntur in, e, v. g. iomi : ióme, lege vel legas. Si vero radices finiantur in, chi, conuextitur in, tc, v.g. machi: mate, expecta. Fit etiane imperatiuum conuertendo, nu, in quo prefens negatiun finitur in, ai, v. g.ex, iomanu, conftituendo, ai, loco, nu, fit, jomai, lege, \& eft modus communis etiam tertic coniugationis; fed ifto imperatiuo vtuntur folum loguendo cum inferioribus 
30 $D E \quad C O N I V G$.

F uturum imperatiui ef futurum abfolutum : v. g. iòmó, leges, ơ illo vtuntur loquendo cum abiectis perfonis.

Reliqua tempora optativsi, fubiunctiui, gerundÿ, infinitiui \&c. funt eodem modo of eifdem particulis quibus in prima coniugatione applicando fingula fingulis etiam in.modis loquendi.

\section{Secunda coniugatio negatiua .}

R Adix negatiua fecunda coniugationis eft conuer $\int, \mathrm{i}$, in 1 quo radix abfoluta finitur, in $2 z u, v . g$. iòmi : iomazu, radix, non legendi.

Prafens fit, fi radix affirmatiua finitur in, chi, illud conuertendo in, tanu, ot, machi : matanu, non expecto. Si finitur in, xi, mutatur in, fanu, ot coròxi : corolanu, non occido. $S i$ alio quouis modo finiuntur in, $i$, illud conuertunt in, anu, v.g. coròbi : corobanu, non cado.

Prateritum eft conuertendo, nu, prafentis in, nanda, 0.8 . corobanu : corobananda, non cecidi, iomananda, non legi, reliqua vero tempora proportionaliter ficut in prima coniugatione negatiun.

\section{Tertia coniugatio affirmatiua .}

R Adices verborum tertic coniugationis finiuntur in, ai, oi, 1 vel, vi, que firticontur in, ai, conuertunt illud in, ó, ad faciendum prafens: v. g. narai : naró, difco . que finiuntur in, oi, vertunt illud in, ô, v.g. vomoi,vomô, cogito, qua verò finiuntur in, vi, illud mutant in, ú, v.g. cui : cú, comedo.

Prateritum fit po/pofito, ta, prafenti : v.g. naróta, didici, vomôta, cogitaui, cúta, manducaui.

Prateritum plusquam perfectum fit conuertendo oltimum, 2, preteriti perfecti in $\mathrm{e}$, br addito verbo, gozaru, in prafenti vel, gozatta, in preterito, vt fupra dictum eft in prima coniugatione: v. g. naró té gozaru, vel, naroté gozatta, iam didiceram.

Futurum fit conuertendo , $i$, in quo radix finitur in , vó, vózu, vel: vózuru, v.g. narauó narauozu, vol, narauózuru difcam. 
Si verò radix finiatur in, oi : conuertitur in , vô, vôzu, vel, vô, zuru, v.g. vomoi, vomouô: vomouozu, vel, vomouôzuru cogitabo.

Imperatiuum fit poftponendo radicibus, e , v. g. naraie, difce, tòie, interroga, cuie, comede. Fit etiam aufferondo à prafentinegatiuo, de quo Statim, dictionem, nu, \& conftituendo loco eizesliteram, $\mathrm{i}$, v.g. narauai, difce, touai, interroga, cuvai, comede, boc modo vtimur cum inferioribus, cateraficut in alijs coniz:gationibus .

\section{Tertia coniugatio negatiua .}

$\mathbf{R}$ Adix negatiua tertic coniugationis eft conuertendo, $\mathrm{i}$, in, qun radix offirmatiua finitur, in vazu, v. $g$. narauazu, touazu, cuvazu, fit vero prefens conteriendo, i, in, vanu:v.g. narauanu, non difco, tollanu, non interrogo, cuvanu, non comedo.

Prateritum fit conuertendo, $i$, radicis, in, vananda, $v . g$. naralla!landa, non didici, tòuananda, non interrogaui , cuuananda, non camedi.

Prateritum plufquam perfectum eft conuertendo, a, vltimum perfecti in, e, đo additaverbo, gozaru, vel, gozatta, v. g. cù uanandë gozatta, vel narauana dé gozaru, non comederam, vel ron didiceram, catera vt in alys coniugationibus,

Poftpofitis verbis fubfantiuis gerundys in, Do, omnium, conits.jationsm tam affirmatiuarum; quam negatiuarum: fit Enfus, $e f$, vel non est factum, quod pergerundium fignificatur : v. g. ãgnete aró, iam erit oblatum, còno qióga caité gczaranu, bic libier non eft fcriptus, āgueíde arózu, nondum obtulerit. Verba verò Jubftantiuc funt, gozaru, gozaranu, voru, uori nai, dea vel gia: dellanai, aru :aranu,vel, gozaranu uoru liòrinai, \& vnumquodque, ex illis fequitur regulas communes fux coniugationis.

Pofpofitis etiam verbis fubflantiuis infinitiuis omnium coniugationsurn fit /en fus quod eft, fuit, erit;vel non, id quod per infinitiuum fignificatur: $v . g$. äguru còto aró, erit boc quod eft offer$r e$ : ideft offeret, naróta còto gozarumai, non addifcet, $\mathcal{O}$ diEta verba Jubfantiua babent omnia fua tempora iuxta fecundam 
32 DE CONIVG.

coniugationem ad quam pertinent, quia corum valicus friuntur in i, ari, $\mathfrak{u}$ : gozari, $u$.

\section{Coniugatio verbi fubftantiui ne- gatiui.}

$\mathrm{V}$ Erbum fubftantiuum negatiuum eft, nai, vel, gozanai, vel, uorinai, quod fignificat non effe, eizus radicie eft, naç̣u vel, gozanaqu , aut, uorinaqu.

Prateritum eft conuertendo, $i$, in quo prafens firitur, in, $c$, \& pofponendo illi prateritum, ari, u, quod eft, atra, v.g. nacatta, vel, gozanacatta, non fait, catera tempora funt coniugando, ari, $u$, per fecundam Secundum temporum exigentiam . fis.

Imperatiuzm ef, nacare, vel, nanaifo, vel, naina, ne

Subiunctiuum eft conuertendo, $i$, prefentis in, qerëba, q. $g$. naqerëba, vel, gozana qerēba, cum non effet vel fit .

Subiunctiuum permifiutim efiz conuertendo, i, profirs in, qerêdòmò , v. g. gozana qcrédòmò , etiam $f$ izon fit.

Preteritum buizus fubiunctiui ef poltpofit, rédomo, preterito indicatizzi : $v$.g. nacatta rëdomo, quamsis non fuit.

Subfantiuum cum particula, tomo, est illam pojitiponcndo radici: v.g. naqutomo, etiam finon fit. Gerundium est, nó, nóte, vel, naqu xitè, vel, nacatte, cum non fit. Catera vt fupra cum verbo, ari, $u:$ fuperaddito, \& coniugatoper fecundam.

Nomina adiectiua quando non anteccdunt verbis, coniugantur.per. Se ficut verbum fubftantiusum negatiuam: illa inquam nomina adiectiua, qua fupra dictum eft finiri in ai, ei, oi, tii, ij, eorum radices funt conuerfa, $\mathrm{i}$, oltima in, $\mathrm{qu}, v . g$. fucacu, radix profiondi, a, iòqu, radix boni, $a$, xíguequ : radix den $\hat{\imath}, a$, uaruiqu, radix mali, a, mali, uonajiqu: radix eiufdem \& $\odot$.

Prafens eft ipfa vox adiectiui : $v . g$. ioi , bonus, a , um, fucai, profundus, $a, u m$, uarui, malus, $a$, um, uonaji, idem,eadem, idem.

Prateritum eft convertendo, i, adiectivi in, c, vel, $\mathrm{q}$, \& $p o f i-$ 
poftpofito verbo, ari, $\mathrm{u}$, illud coniugando fecunduin exigentiam orationis in omnibus temporibus.

Permifizuum cum, tomò fucàqu tomò, vel, fucải tomò , quimuisprofundum.

Girusidium in, do, fucóte, eum effet profunctum, ióte, cum fit bonum, varite, cum fit malum, cana xiute, cum fit trijte, xingueo te, cum fit denfum. Sunt etiam, fucó xite fucaqu xite, vel, fucacdtte, ơ fic in alÿs: $v . g$. iôxite, iòqu xite, iocatte.

Adiectiua finita in na, nose coniugantur; gerundia tamen in do, folent babere : $v . g$. aqiracana, pro gerundio, a qiräcani xite, cùm eflet clarusn; idem, aqiraca de . arifóna, babet arifoni xite, cum fit apparens vel verifimile, iónà, babet, ióni, v.g. iòi yóni xitè , cum fit boni modi, vel babeat bonum modum, càvãga fucóte vatarananda, quia flauius er at profundus non. tranfúadaui, xebòte irarènu, quia Arickum, non eft intrabile, varute cu varenu, non efl comeflibile vel non poteft comedi, quia malum. Catera tempora adiectiuorum funt ot dictum eff, cum verbo, ari, $u$, coniugato fecundum exigentiam orationis. Coniugatio etiam negatiua eft cum eodem, ari, $u, v . g$. radix eft, fucdcarìzu, prefens verò ef, fucdcarì nu, non eft profundum. Prateritum, fucacaranda, non fuit $\delta c$.

\title{
De particulis conditionali- bus.
}

\begin{abstract}
Q Vinque funt particule facientes orationem : conditionalem, naraba, ni vòite va, raba, va,ba, dua fecunde po/ponuntur omni verbo tam affirmatiuo, quàm negatiuo is prafenti, praterito, \& futuro, \& cum illis remanet verbum conditonale: ข. g. nígưru narabas $\sqrt{2}$ fugis, iôda ni uòite va, $\sqrt{2}$ legifis,nara vó naraba, $\sqrt{i}$ difces, cú vadu ni vòite va, $\sqrt{2}$.non cumedis, aliquando tollitur voi,$\dot{a}$, ni voite, $v . g$. ãgueô ni va, $\sqrt{2}$ offeres, ägueca ró ni va, fa obtuleris. Tollitur etiam aliquando, voite, b remanet folum, ni, v. g. mairó ni còlo, nèn gòrò ni mòlozure, fi ibo vel jerim fignificabo illi amicabiliter, xitaró ni còfo,


34

D E C O N I V G.

faifòcu tçùqu maji qerè , î feciffem; non babuiffet effectum, diligentia of perfuajio.

Particula, raba, poftponitur preteritis: v. g. narŏta raba, fi didici fem, narauananda raba, $\sqrt{2}$ non didiciffem .

Particula, va, postponitur radicibus negatiuis omnium trium coniugationum; $v . g$. ãguèzu va, fi non offero, iomazu $\mathrm{va}, \sqrt{i}$ non lego, narauazu va, $\sqrt{i}$ non difco, naqu va, $\sqrt{i}$ non eft, fucacarazu va, finon e/fet profundum

Particula verò, ba, babet eumdem efféctum or iungitur etiam radicibus, quibuis. va âguezúba, iomazúba narauazüba, fi vero dicta particula, ba ponatur loco, $\mathrm{zu}$, radicibus negatiuis, fit conditionalis affirmatiua oratio: v.g. ãguéba, $\sqrt{2}$ offero, iomãba fil lego, narauãba, fi difco, iocaraba, fieft bonum. particula vero, ua, non folum poftponitur radicibus negatiuis adiectiuorum: Sed etiam affirmatiuis: v.g. fucaqu ua, $\sqrt{3}$ esprofundum, uonajiqu ua $\sqrt{2}$ est idem. aliquando boc verbo vtuntur ac $\sqrt{2}$ dicant: fi non eft valde moleftum : facias bor. dicunt etiam, ãgue majiqu ua, $\sqrt{2}$ rron offeres.

Particula, ni uòite ua, fupra pofita iungitur etiam aliquando nominibus, \& quafi fupplet verbum fubftantiuum: v. g. jó jó nì uoite ua uqe toró, accipiam $\sqrt{2}$ eft valde bonum vel optimum, curùxicarazaru guì ni uòite ua,$\sqrt{2}$ non fuerit moleftusn vel res molefta.

Particula, faie, pofita in oratione, obi eft aliqua particusla ex conditionalibus fenfui orationis additvirtutem: $v$. g. fune faie mairu naraba, $\sqrt{2}$ venerit aliquod nauigium, fonata faie uocutabire naqu ua, fi non eft defe/fus, ac fi diceret: ex mea parte, vol quod ad me attinet ego non fum defe Jus.

Supplet etiam aliquando particula, faie, conditionalem: v.g. Niffon no xốco cu ni faie caióna còtò gozaru fódonì , fă ergo in regno paruo Iaponic inueniuntur \& Sunt res buiufmodi, ac $\sqrt{2}$ dicat;quanto magis erunt in magnis, còco mòto no tocai ni faie mei uacu itafu ióni gozaru fódoni \&c. fi ergo in nauigationibus, que bic funt, valde patior ơc. fito faie côquai fưru mòno uò iurufu ni iuan ia, Deus ni uoite uoia ? fi ergo bomo ingnofcit bomini poenitenti, quanto magis Deus? còre födo xei uo iru ru faie còto naricanùru ni ; ucato xite ua, incãdeca banji canauozo ? Ji tot adbibendo vires vix potui fieri ; fi leuiter fui fet factum quomodo potwi Jet fieri feu funiri ? còre faie xinicui ni, $\sqrt{2}$ 
ergaboc efl difficile, fune de faie ioio tçuita ni,cachi una nananaca naru mai, fi naurgio vix perueni ; pedes abfque dubio non potuiffem.

\section{De verbo potentiali .}

D Ofpofita particula, ro, prefentibus of futuris verborum il1 la facit potentialia: v.g. aguru ro, forfan offert, nigueozuro, fortafis fugiet.

Praterita fiunt conuertento, $\mathrm{ta}, \mathrm{in}, \mathrm{t} c ̧ \mathrm{u}$, \& addito, ro, $\boldsymbol{\sigma}$.

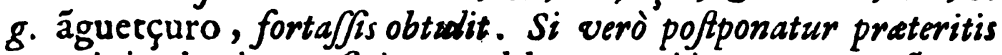
negatiuis, da, in quo finiunteur, debet mutari in, zzu, v. g. ãguenanzzuro, pofibile cfit non obtuliffe, vel quod non obtulerit, vel obtulit.

Fit etiam potentiale prafens pofpofito, arozu, vel alio futuro, infinitiuo: v.g. ãgùru còto mó aròzu, vel, ãgue mò xôzu, forfain offert.

Preteritum eftpofpofito futuro praterito: $v$. g. ãgueta còto mo arozu, forfitan obtulit.

Futurum, âgueô còto mo arozu, forfan offeret, idem etiam eft in negatiuis: v.g. ãguenu, vel, äguenanda, vel, ãguru mai còto mo arozu, pofibile eft quod non offert, obtulit, vel offeret, ô quando volumus dicere, ita erit : loco, còto, ponimus, mono,v.g. noxenanda mono dearózu, fortafis non introduxerunt in nauigium, iqi chigota mono dearozu, non fe obuiauerunt in via, moreqicoieta mono de gozaro ca to zonzurù, credo $\sqrt{z}$ forfan est diuulgatum.

Ad fignificandum fierifignificatum nominum adiectiuorum pofponitur verbum, nari, $\mathrm{u}$, coniugatum fecundum exigentiam temporis ipfis adiectiuis aduerbialiter fumptis: v. $g$. fuco naru, fit profundum, uaru natta, factum eft malum . dicitur etiam fuco aru, eft profundum, aliquando etiam dicunt, fuco nai, now es: profundum, \& boc modo loquendi vtuntur etiam coniugasdo nai, modo fupra dicto fecundum exigentiam temporis, iuxta fenfum orationis. etiam dicunt potentialiter, fuco nai coto mo arozu, forfan erit boc, quod eft, non effe profundum.

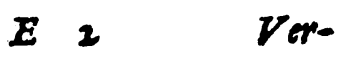




\section{Verba irregularia quo ad coniu- gationes.}

$\mathrm{V}$

Erbum, qi, uru, quod eft venio, is : babet prafens, quiru, venio, qita , veni, côzu, venian, coi , vel, coio, vzni, qitarëba, cum venerit, vel א̧ veniffet, qitarédomo, quăuis venit, ơ radicem negatiuam, côzu, ơ prefens negatiuum, conu, non vexio, mède radix verti delectar, aris, babet prefens mézzùru, or gerundium in do, medete, delectando fe, cui, radix verbi paniteo, es, vel triflor, aris, babet prafens, cuiuru, o gerundium in do, cuite, paniterids, of radicem negatiuam, cuizu, \& prafens negatiuum, cuinu, mon panitet, araie, radix verbi fum, eft, fui : babet prefens, araiuru, fiue, aroru, eft. furi radios serbi vetexafco: babet prateritum, furita, inuetcratus ef , \& gerundium in do, fürite, inueterando, fe, rodix rerbi tranfeo, is, babet prafens, furu, tran fit ; or preteritum, feta, tranfit, Tari , $\mathrm{u}$, efl verbum Jignific ans rems elfe completarin ef integram: babet prafess, taru, Jufficit, prateritum, tatta , sompletum fuit, of futurum, tari maraxo, erit perfectum vel fufficict: \& radicem negatizam, tarazu, preferss regatiuzm, taranu, preteritum, tarananda, non fuit ufficiens, futurum, caru mai, non erit fufficiens, imperfectum Jubiunctiui taravëba, cum non fufficeret.

Permi fiuum, tarané domo, infinitiuum negatiuum, taranu coto, gerundium verd in do, caraide, vel, Tarazu xits, verbum taxi, tafu, quod fignificat adimplexe, feuperficere, babet futurum, taxi marâxô, perficiam, tấann, vero eft eizss trefens negatiuum. Tari, autem radix verbi, taro, quod fignifirat e Je perfectum, babet pratexitum negatizum, cara uananda, non fuit penfectum, of fubiüctiun, tara uanéba, cum non. effet perofectum, of permiffissum, tara uane domo, or infinitizum, taraluanu còto, of genundium in do, Tarauaidc, vel Tarauaxu xitè : uocotari, vero ef r radix verbi, uocotaru, pro so quod eft deficere: babet infinitiusm, uòcotaru coto, ơ radicem negatiuam, wocotarazu, \& prefens negatiuum, vói cotaranu, voi,ef radix verbi quod babet prateritum, uoita, inuetera- 
tuss eSt : \&o, uoitaru, quod eft idem. Et prafens negatiuum quod ef, uoinu, of gerundium in do, uoite : urei, radix verbi tri-Aor, aris, babet prefens, vreô, ơ imperatiuum, vreio, ơ infinitiuum, vreoru coto, of gerundium in do, vreite: Tomi, radix verbi, tomu, vel, tomeru quod est ditor, aris, vel diues feri, babet preateritum, tonda, gerundium in do, tonde, 0 radicem matiuam, tomázu, fai guiri, $u$, fignificat idem quod praeo, is, vel anticipor, aris, babet prateritum, fai guitta, or gerundizem in do, fai guitte.

\section{De verbo adhuc, \& de eius formatione $\&$ differentijs.}

$T$ ifta lingua funt verba actiua implicia; of actiua etian 1 faciendifacere, paffiua etiam, neutra, or imperfonalia. Omnia verò coniugantur per tres coniugationes fuprapofitas $f-$ cundum quad eormm radices iam difpofite of exillis verba for mata, terminantur.

Ex aliquibus nominibuss adiectivis aliqua procedunt verba: v. g. catài , eft dourus, a, uns, ex quo exeunt, catàme, uru, induro, as, actiursm, catamari, $\mathrm{u}$, indure $c 0$, is, neutrum, of carameràre, uru, induror, aris, paffiusum, ex canaxii, adieEtiuo quod efl triftis, e, exit, canaximi, u, quod est tristor, aris.

$V$ erba faciendifacere, formantur iftis particulis, fáxe, vel, xe, prima poftponitur radicibus Secunda coniugationis; fecunda vero radicibies feeunda, \& tertice, fiunt autem postpofitis presfentibus negatiuis, auferendo nu, in quo finizontur do porsendo loco eizes pradictas particulas: v.g. äguefaxe; uru, offerre facio, is, iomaxe, uru, legere facio, is: naraudxe, ura, difcere facio, is, of omnia remancnt feeundo côinsgationis quia particule finiuntur in, $\mathrm{e}$; aliquando etiam, $\delta \sqrt{2}$ rara, folet pofponi particula, faxe, verbis fecunde, of tertia coniugationis, fod tune ornantur fex bonorantar predicta verba cum particula, rare, v. g. iomafaxe rare, uru, Padre ua dòjuctini cathecifmo vo narauafaxeraruru, Pater iubet fuo minifiro vt difcat catbechif-

mum 
mum, mòno no fòn vo fito ni iòmafaxerarùru, facit legere originale.

Verbapafjusa fiunt particulis, rare, of re, particula, rare, iungitur actizuis fecunde coniugationis modo iam ciicto tollendo foilicet, nu, a negatius: :v.g. ãguerare, uru, offeror, eris, iomare, uru, legor, eris, nara vare, uru, difcor, eris, bis vtuntur in fen fue paffiuo legi ab alio, vel efle, aut non e/fe.legibiles: v.g. funt etiam alia paffiua qua procedunt ex neutris vel ex babentibus fignificationem veutralem, que quidem formantur cum particulis, rare, of , re, formata.tamen non regunt cafus cômunes pafjuorum (de quibus infra) fed verborum ex quibus procedunt : v. g. ex, ãgari , u, procedit, ãgarare, uru, \& quia, agari, $u$, quod/fignificat afcendo, is, regit accufatiuum, etiam illum regit, ãgarare, uru, v.g. cono iamaie ägararenu, non potefl afeendi ad ifum montem. vel ifte mons non est afcendibilis, xiròcara derarenu, non poteft exiri ex́ caftello, Xebóte irarènu, nö poteft intrari quia frictum fou anguftum, còno michi va arucarenu, non poteft ambulari bac via, natçu vacòco ni irare mai, non erit boc babitabile tempore veris, còno füdé de va cacarenu, non poteft $\int$ cribi isto calamo, fimäga nóte cacarenanda, non potuit foribi ex defectu temporis, cònobùn ni còfo cacaruru mc̀no de gozare, boc fane modo bene fcribitur, axíga itóte aru carenu, non poteft ambulari dolentibus pedibus.omnia ergo verbe paffiua funt fecunda. verba neutra funt que babent fignificationem neutralem : v.g. aperiri per fe \& non ab alio : v. g. ivógatoruru, pifces capiuntur, cajëga torùru, ventus ceffat, itóga qiruru, filum rumpitur, jiga iomùru, litera benè legitur, aqi , $u$, aperior, iris, qiri, $u$, est fcindo, is, actiuum, qirare, uru, ef/ findor, eris, pafjusum, qire, uru, eft foindor, eris, neutraliter eft etiam quando gladius berre findit quia eft acutus, qiraxe, uru, eft verbum faciendi factre quod fignificat fcindere facio, is, ägue, uru, eft leuo, as, ãguerare, uru, leuor, aris, paffiuum, aggue faxe, uru, leuare facio, is, ãgari, $u$, leuor, aris, neutrum, ãgarare, uru, edfe afcendibile, ãgaraxe, uru, leuarifacio, is, vel quod fe leuet facio, facis. $f i$ verò illis adiungantur particula bonoris (de quibus infra) faciunt alias combinationes. adiectiua verò quando coniugantur babent fignificationem neutrulem : v.g. fidarui, efurio, is, fucacatta, fuit profundum .

$V$ erba imperfonalia non nominant, neque exprimunt perfo- 
DE C O N I V G.

nam : v.g. mi uo fatafu tomò itçuvari vo iuanu mono gia, etiam $\sqrt{i}$ quis moriatur non debet mendacium dicere, mòno mò tabezu faqe mo nomaíde ichinichi fataraqu mòno ca? potest ne laborari per totum diem integrum nibil comedendo fo non bibendo vinum ? Xujin nò maiể de fòna ióna còto vò iú mono ca ? polfunt ne dici buiufmadi coram Domino? Quoad coniugationes verd fequuntur regulas radicum quibus efficiuntur.

Radices omnium verborum cuiufcumque fint coniugationis, pofunt adbuc extrabi o deduci ad alias ecniugationes fi illis fuperaddantur particule bonoris, fecundum literas, in quibus predicke particula bonoris finizuntur, particule verò funt maraxi, uru ari, u faxerare uru, xerare uru, nafare uru, faxemaxi u, tamai ó,rare, re.

Particula, maraxi, nonaddit bonoren rei de qua loquimur; fed loquimur bonorate attendendo ad perfonam coram qua loquimur: v.g. cui ú, Jignificat id quod comedo, is, feruus autem. coram Domina non dicet, ièzumi gacúta, mures comederunt cafeum: v. g. Sed,nèzumiga cui maraxita, notandum etiam quod cui ú, fecundum fe eft tertic coniugationis quia finitur radix in, vi, addito verò, maraxi, redditux prima. quando referimus aliquid de aliqua natione verbum non bonoramus; fed folium attendimus ad perfonam cum qua loquimur ad addendum illi vel non particulam feu verbum, maraxi, uru, v.g. coram inferiori dicemus, Nan ban jin va còre vo cuvanu, coram perfọna verò nobili dicemus, Nan ban jin va core vo cuvanu, Europeiboc non manducant, Ari, u, pofponitur radicibus omnium verborum; \& illa bonore afficit mediocri: v. g. módoriaró ca? reucrteris ne? Si verò anteponatur illis fic confitutis, vo, bonorantur verba fatis: v. g. vomödori aróca 3 reuertetur ne veftra dominatio? Tono fama vo xini atta toqi, quando dominus mortuus eft, Deus cono xecai uo gofacu atta, Deus creauit bunc mundum, bis particulis vtimur loquendo cum per fonis bonorat is quas diligimus, \& cum quibus babemus amicitiam .

Particula, nafare uru, bonorem fupremum, ast $\int a t i s$ magnü dat verbis; poflponitur verò eorum radicibus: v.g. Deus cono secai uo ge facu nafareta, Deus creauit bunc mundum.

Particule, rare, of re, bonorem quidempreftant lignifintis, verboruin, quibus adduntur; fed mediocrem; or non magnam: pofponitur autem, rare, \& pracipuè fi loquainur de 
40

D E C O NIVG.

abfentibus, prefentibzs negatiuis ablato, nu, of conflituendo particularn prediczam eius loco: v. g. ãgue rare, uru, eft offero, ors. quando offerens eft peryona mediocris bonoris du reuer intice coincidut cum pafiuis in literis; fed cafibus qucos regunt, diftinguuntur. particula, re, poltponitur verbis jecunda $\dot{0}$ tentice codem modo: v. g. iomare, uru, naravàre, uru, legere of difcere perfonam boni nominis, boc modo loquimur de aqualibus \& Seruus etiam de domino fuo,izon cum conferuis; fed cum gente nabili.

Particula, fàxe maxi, $\delta$, xemaxi, eundern trikuunt bonoress quem, dri, $u, \delta$ quem, rare, $\delta$, rc, postponitur, faxe máxi, $u$, radicibus fecunde, vel prefenti negatiuo, ablato nu, br constituto, faxe mixi, $u$, loco eiuls: v.g. ãguefaxe màfu, offert, maxi, u, vero po/tponitis negatiuis fecunda of tertia, ablato, nu, v.g. iomaxemafu, legit, naravaxe malu, diScit.

Particule, faxe rare, uru, xerdrc, ur:1, magnum tribusunt bonorem, prima poftponitur prafenti negatiuo verborum fecunda: coniugationis, ablato $\mathrm{nu}$, fecunda verò jofponitur negatiuis fecunde or tertice eodens modo: $v . g$. ägue faxe raruru, offero, $r s$, iomaxe rarùru, lego, is, naravaxe rarùru, dif $\mathrm{co}$, is, quia, vero bac coincidunt in literis cum verbis faciendi facere bonoratis; ad tollendam aquiuocationem, vtimur particula, ari, $u$, antepofita, vo, verbis.: v.g. y.omaxe aru, .lego, is, nara vaxe aru, $\operatorname{dif} c \theta$.

$V$ erba pa/fiua, de quibus infra, po/funt admittere particulam, faxe rare, uru, v.g. via mavare faxe raruru, bonoror, aris .

Particula, tamai, ó, tribuit Jupremum bonorem: illa vtimur loquendo de Deo, fanctis, regibus, or imperatoribus. postponitur vero radicibus verborum, que efficit tertic coniugationis. poftponitur etiam radicibus palfiuorum loquendo de Deo: v. 8 . Deus filio, vmare tamò tòqi, quaindo Dei filius natus eft, Deus ägamerare tamo, Deus bonoratur.

Particula, tate matçuri, $\mathbf{u}$, bumiliat.Jgngificat um verbi cui adiungitur: poftponitur autern radicibus verborum affirmatiuo$\mathrm{rum}: v \cdot g$. Deus vo gotaixetni zonji tate matçuru còtova ichi sūgureta jèn gia , amare Deum eff fuprema virtus : amittit tamen bec particula bonorem is particula, re,mutato $\mathrm{e}$, in quo finitur in, 2, v. g. loguendo de Janctis respectu Dei dicemus Sancio Do- 


\section{DE CONIVG.}

mingo, Deus vo gotaixèt ni zonji tatematçurareta, Sanctus Dominicus dilexit Deum .

Particula eticm, màráxi, poteft ad bonorem eleuari particula , rare, v.g. tòno iòri cónó còto vo Padre ni vatàxi mai raserareta, Dominus tradidit banc rem patri.

\section{De aliquibus verbis qux de fe habent honorem. determinatum.}

M Efare, uru, fignificat facere quamcumque actionem quam poteft, \& eft decens facere perfonam nobilem, ot eft comedere, bibere, nauigare, equm afcendere orc. vôxerare, uru, $\sqrt{2-}$ gnificat loqui perfonam nobilem, uomaraxi, uru, vomaraxi, ari, u, fignificat dare perfonam nobilem, woxe, uru, $\mathcal{W}$, uôxe ari, $u$, fignificat loqui vel pracipere perfonam mediocrem.

$V$ erba quibus anteponuntur, uôxe, vel, mexi, eundem babent bonoiern cum illis; ऊ abfque illis: v.g. uôxe tçuqerare, uru, quod eft pracipio, is, $\mathcal{W}$, mèxi tçuca uare, wru, quod eft Sertsio, is, est idem quod, tçuqerare, uru, $\delta$, tçuca uare, uru, ad vocandum imperatiue dicimus, coi, feruo vel inferiori, iòrài, dicitur non tam inferiori, waxèi, eft aliquantulum inelius, úogiare, eft fuperior modus vocandi, gòzare, veniat vefrra dominatio, gozaro, vero in tempore futurieft bonorabilior modus quia eft fine imperio, uoíde nafarei, vel, uoide nafareô, vel, woide nalarei caxi! eft veniat v'efra dominatio: vel, oj $\sqrt{z}$ veniat vefira dominatio ! cudafare, uru, fignificat dare perfonam nobilem, :amauari, $u$, dare perfonam nobilem inferiori, tamóri, $u$, dare perfonam mediocrem, mizzu uo nomaxète tamore, da mibi bibere aquam, cudafare, uru, $\delta$, tamóri, $u$, fignificat comedere perfonam bumilem cibum bonorando. còxi mexi, $u$, $\mathcal{W}$, qicoximexi, $u$, eft comedere, vel audire perfonam nobilem, uoboxi mexi, $u, \delta$, uoboxi mefare, uru, cogitare perfonam nobilem, faxerare, uru, facere perforam nobilem $\mathcal{O}$ idem nafare, uru, afobaxi, $u, \mathcal{G}$, afobafare, iuru, $\sqrt{i-}$ gnificat facere perfonam nobilem quidquid illi esf decens: v.g. venari, fcribere legere, recitare, ii , $u$, eft loqui bnmiliardo loquentem, $\delta$ rem de qua loquitur, $\mathcal{W}$, niexi, u, fignificat etiam 
Loqui bonorando perfonam, ơ rem de qua: onde non recte dicam mi ni móxe, dic mibi ; fed, mi ni iie, neque dicam, tono ni iie dic domino ; fed, tòno ni mòxe . mairi, $n$, figuificat ire ad locum cuibonor debetur: v.g. iglefia ie maire, eas Ecclefiam, cuire, uru, , tordxe; uru, fignificat dave, bumilicsndo perfonam. cui datur, cui, ǔ, eft comederc fine aliquo refpectu, mexi, u, eft etiam comedere; fed eft urbanum : $\sigma . g$. coram bonestis non dicäm, mèxi uo cùi maraxita; fed, mèxi uo tabe maraxitá, comedi, mairi, u, vel, uomairari u, eft comedere perfonam, nobilem vel mediocrem, ägara xerare, uru, ơ, uoâgari ari, u, ef modus sobilior, qiqi, $u$, ’́est audire ot cumque; uqe taan uari ,u, vero er, uqetamóri, $u$, eft audire bonorando perfoncrs dqua auditur: v.g. goiqen uo uqetamòtta, vestra confilia. audiui, móxi ãgue, uru, eft loqui bumiliando fe loquentem, \& bonorando perfonam cui dicitur, móxi ire, uru, loqui inter wquales, chómon xi, uru, audire fermones Dei, goiànji,zuru. vel, goranjerare, uru, est afpicese rem nobilem, xi, uru, of facere in communi, itaxi, u, eft facere; fed dicitur modo orí.3no, tçucamatçuri, $u$, est focere, bumilisndo fe quifacit,

\section{Aduertentix circa coniugationes ver- borum .}

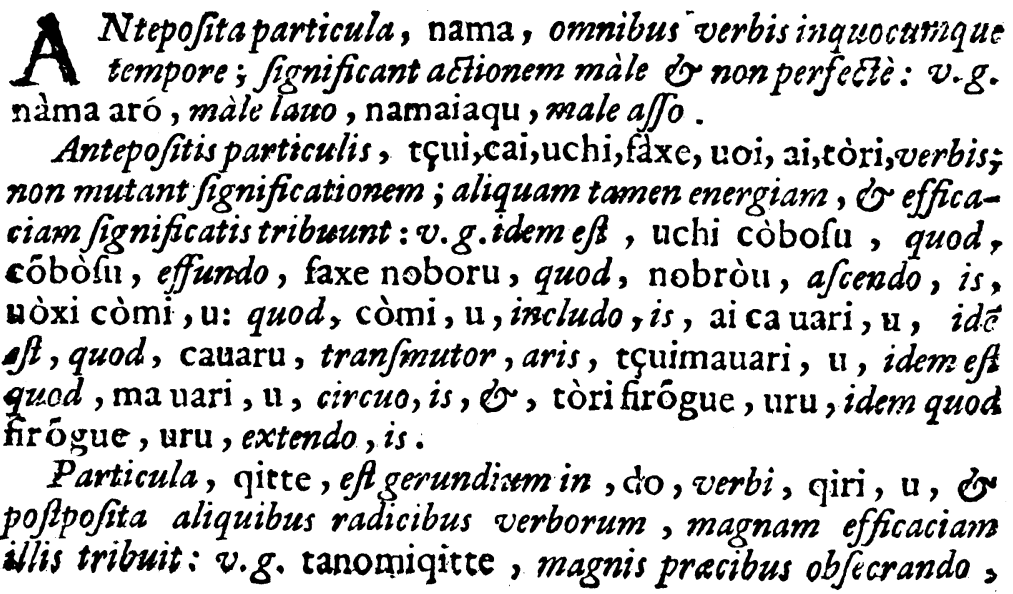


$D E C O N I \vee G$.

nomòi qitte, magnam affumendo refolutiontevitimus etiam verbis, tanomi qirs, $u, \mathcal{O}$, uomoiqiri , $u$.

Antepofita particula, ma, aliquibus nominibus feu verbis dat fignificato vigorem: v.g. mamucdi, valdeprafens, macuròi, vialde nigrum.

Particula feu radix verbi, macìri, $\mathrm{u}$, antepofita verbi: fignificantibus motum facit verba modesta, of vrbana fatis: v.g. macari noboru, afcendo, is, macari cüdari, u, defcendo, is, macari i, iru, ad fum : es.

Antepofita particula, na, in oratione confirmat id quod antea dixerat quenfi gloriando fe illud pradixi/fe : v.g. fune ui cuchi notçu ie iru uà,nauigium intrat, cuch hinotçu, ac fi dicat, nonne:To bene dicebonn? aru ua, vide $\sqrt{2}$ eft 2 icut ego dixi!

Particula, aidani, $\int$ gignificat inter, idefi tempus quod in aliqua aEiione confumitur: v.g. ãgura aidani, inter offerendum, vel sium offert, iôda aidani, dum legit, narauózuru aidani, dum difcet.

Particula, ga , fgnificat , fed:'v.g. fóiú ga ; nanto aró ca? fic dicunt; fed quomodo erit vel fi erit nafcio certe, füri ua furru maiga, fune uo dafu còto naró ca xiranu, profecto non pluct; fed nefcio vtrum poterit extrabi nauigium, fono qinpenni ua gozaru maiga ; dôco cara toraxerarưru zo? non crunt apud wicinos vel in circuitu, onde ergo afferunt?

Particula, gotòqu, polpofita prefentibus, preteritis of futuris fignificat, eo modo quo: v.g. coxiraiùrú gotòqu, ficut, vel eo modo, quo ornas vel porficis, qiita gotòqu, ficut audiui, aliquando eft, gã gotòqu, v.g. móxitã gã gotoqu, ficut dixit, caraca uózú gotóqu, comodo quo contendam vel rixabor idem facit particula, ioni, v.g. Nifon no catãgui uo xirareta ioni, uôxerarùru, loquitur ficut qui fcit confuetudines Iaponia, mófú ióni, ficut deco, vtuntur etiam particula, furt, ad eurdem finem v. g. Toza no chijòcu uo nogareô zuru tameni catana uo faitz firri uo mixerareta, oflendit fe accinctum gladio, vt euaderet imminens infamia periculum, minu furi uo faxerareta, oftendit Se non vidifis

Particula, faie, vtuntur ad fignificandum nec dum: v.g. mma faie nacatta, equi nec dum fuerüt, cotóba (aie xiranu mono, neque loqui fcit, ji faic mixiranu mòno, nec dum literas cognofcit vtuntur etiam eadem particula ed exaggerandum aliquid

$$
F=\text { v.g. }
$$


44

D E C O N I $\vee$ G.

v. g. qiden to faic moxëba, fufficeret fi diceres te effe, Padre ro tçucauaruiru to faic, móxéba, $\mathfrak{f}$ dixiffet folum quod patrifer. uiebat: ac fi diceret : boc fufficeret vt $\delta c$.

Particula, qere, eft confirmatio \& terminatio orationis, \& fignificat; itaque: v.g. maitta qere, venit itaque, fate fóaru qere, res denique fic fie babet.

Particula, còfo, eft magni momenti inter Iapones vtuntur enim illa in primis in fenfu aduer fatiuo : $v$. g. còre còfo ió gozare, boc eft verí bonum. fi oratio inqua inuenitur predicta particula finiatur in verbo, tale verbum finitur in, $\mathrm{e}$, vt in oratione pofita: $\sqrt{2}$ autern verbum fit in tempore praterito additur illi, re, v. g. iô còfo gozatta re! bene veneris! deficit bec regula vel quando cratio non finitur in verbo aut adiectiuo: $v$ g. còre còlo xixó yô, bic efi verus magister:vel quando poft particulam, cò 10 , eft in oratione gerundium finitum in, te, vel permiffutum ct:m particula, tomò, aut praterita potentialia finita in, tçurǒ, ciel, z zuró, v.g. uare cofo iro iro xinro tçicamatçùtte cutatireba toxiiórini nari maraxita,pationdo muitos \& diuerfos labores vere factus fum senex defeffus, of laffus, ware còfo coro!aruru tomo, ego enim \& fioccidar oc. fara còlo ta:tçuro forfam fuit iratus, fato chicaqerêba cốc fin. miinre : videtur iamignis quia prope eft vicus. bac oratio finitur in e, quia non efü:? ea regula exceptio, uôxerarèta coto domo no go côquaí de cofö gozarózure, abjque dubio facietis poenitentiam de üs qua dixiftis, cataji qenò colō gozare, tibi valde congratulor do gratias ago. quando aliquis interrogat quis fecit boc? reffondcnt: $v \cdot g$. Patre coío, Pater fecit : ac $\sqrt{i}$ dicant: videte $f i$ eft perfona qusecumque, que illud fecit? \& quando quis responfum non auditit aut percepit, o iterum interrogat, dicit qui loqutus $\mathrm{e} f$, juan cofo, iam dixi, quod Ioannes ¿ 6 .

Quando non curat quis deïs qua illi dixerunt, vel babet $\int e$ ac $f i$ non audiffet vel iterum interrogat, folent refpondere: $v$. g. touoru na toiiēba, iam dixitibi ne iranjeas, iome to iiëba, iam dixi tibi quòd legas, Padre cofo to iieba, iam dixi quodpater eft qui éc.

Maieni, vel, faqini, pofpofitum prefentibus negatiuis, facit alla affirmatiua: v.g. iglefiaie mairanu maie ni, antequarn eat Ecclefiam . etiain folet poftponi futuris affirmatiuis: $v . g$. mairozuru tote nofagi ini, tanti/per antequa m veniret. 
Particula, tocoro, Jignificat tempus in quo fit actio Jignificata per uerbü cui pofponitur:taburu tocoro ni, quădo comeds $\vec{a} \overrightarrow{\text {, }}$, tabeta tocoroni, post prandium, tabeôzuru tocoró ni, vel, tabeôzuru ni, quando eram comefurus: facit etiam eadem particula reduplicatiua denotando reduplicationem in quantum: v.g. jefu chrifto humanidad no uon tocoro ua, Iefus Cbriflus in quantum bomo, uonoréga foxxezaru tocoro vo fódocofu coto nacare, quod tibi non vis, alteri ni facias, füdai no tocoro no uo iurúfu, dono illi libertatem, fito no acu no tocoro ni ua dôxin xenu, non confentio bominum peccatis, utagó tocoro ino nai, non remanet locus dub ÿ vel dubitandi, nocoru tocoro mo nai, nibil amplius restat, tçuini,fòno tocòro ie mairózu, denique ad bocperueniet, fümbet nj voiobanu tocorogia, res funt qua non intelliguntur, vel ad quas intellectus non peruenit, nani mo naì tocoro vo iô qicòximexe, comedat veftra dominatio ex bac paruitate, qua eft nibil. exemplis cognofcetur vis Ignifisaticnis.

Particule, tocòro,Made, vel, made de gozaru, folent pofiponi ad cadentiam; abfque aliqua fignificatione, \& idem eft, còto de gozaru, v.g. naranu made, vel naranu còto de gozaul, eft iddem quod, naranu, none eft po/fibile, guijèt tçucanatçuro to zonzuru còto va cacúgo ita lanu coto gia, amicitiam fri:iz gere neque in mentem mibi venit, bic, ita fanu coto gia, eft idem guod, jtafanu, folum.

Particula verò, madeiò,otuntur aliquando ad confirmationem enrsim, que dicunt: v.g. caita madeio, quodfcripfiscri$p^{\prime i}$.

Particula, toqi, poftpofita prafentibus, illa facit praterita, imperfecta : v.g. jennìn tachi va faígo nì voiobi tamó tòqi va buji ni gozatta,quardo Sancti perueniebant ad mortis boram erăt pacifici orquieti.

Convertendo, ta , proteritomum in, tçu ; \& , da, negatiuorum in, 2zu, fit fenfus ; modo facio boc; modo illud: v.g. mòno vo caitçu, iôzzu, nando xite curafu bacari gia, legendo, \& fcribendo, E alia faciendo, tranfigo vitam, tatţ̧u itçu vocu iori zaxiqi ie íde zaxiqi iori vocu ie iri xitten battò xeraruru, Ran.do o fedendo intrando, \& exeundo, furgit \& cadit.eumdem fenfum facit particula, ri, poftpofita prateritis: v.g. xeqen nò mòno va netari vogitari nôdari curafu bacari gia, bomines müu-

di, 


\section{DE C O N I G.}

di, vitam agrut dormiendo, furgendo, \& $\sigma$ bibendo, mazzu ite ni ua uo mo tacaxetari, cura vo mo ficaxetari iroiro no xigoto vo atégote cofó mairozure, ibo do atrium verrere faciam, of berbas euellere, of denique ibo ad multa difponenda, ima còno io fuqe iuqéba nome ia, vtaie ia fitó bibo mórçu, vtotçu facamori furu, cum iam fit alta nox proulocando fe ad bibendum ob. cantandum letantur bomines faltando of cantando orr.

Particula, ie, que eft radix verbi, ie iuru, quod est poffum, es, antepofita negatiuis fignificat non po ofe facere actionem fignificatam perverbum: v.g. ie iomanu, non polfum legere, infinitiuis vero postponitur: v.g. iomu còto voienu, non pofum legere. dicitur etiá iomi va ieide, oel iomi mò ieíde, cum legere non poffim aut legere non valendo. Infinitiuum gerit vices aliquando fuppofiti verbi: v. g. xinuru cotova voloròxij, terribile efimori.

Particula, tai , qua fignificat volo, is, poftpofita radicibus verborum Jignificat velle facere actionem /gnificatam per verbum: v. g. mizzu vo nòmi tai, defidero aquam bibere, idem, mizzu uo nomi tó gozaru, vel, mizzu un nomi tó zonzuru, fed ifte duce vltima funtnobiliores, \& quibus coram nobilibus vtimur negatiui exernplü eft, tomo nai, v.g. mizzu uo nòmi tòmo nai, nolo aquam bibere idenz eft, mizzu no nomi tomo gozaranu, mairi tó mo zonjenu, non babeo animum eundi . Si verò particula, tai, fofponitur adiectiuis vel verbis Jignificantibus aEtioncm fexf fitiuam in fecunda perfona, conuertit, $i$, in , $c$, o pofponitur verbum, ari , $\mathrm{u}$, coniugatum fecurdum oraticris exigentiam: v.g. cui tacatta, volui comedere. Si vero verbum loquatur de fecunda, of tertia perfona conuertit particula,, $\mathrm{i}$, in, $\mathrm{g}$, \& pofponitur tiam, ari, $\mathrm{u}$, vel cum bonore fecundum quod per/ona meretur; vel fine particula bonoris; fed abfolute. Si vero fit perfona inferior etiain $f \hat{s}$ fit fecunda vel tertia , i, conuertitur in, $\mathrm{c}, \mathrm{ficut}$ dictum eft de prima.

Particula, de, aliquando facit fubiunctiui fenfum adiuncta aliquibus nominibus fubftantiuis: v.g. uaräbê de xinda, mortuus eft puer vel cum ad buc effet puer, uarëga buchó fóde tofo mo gozanai, cum ego fim negligens, ơ non curiofus, nibil erit, co modo quo conueniebat, aptatum.

Particula, io , que fignificat modum, poteftponi radicibus verborum, of ip/is etiam oerbis: quando radicibus, regit geniti- 
utum; quando vero verbis regit corum cafus: $v . g$. cono qio no iomi iŏv a, modus loquendi bunc librum, vel, cono qió uo iomu io ua, in prima oratione, qio, eft in genitiuo cum particula, no, in fecusda autem eft in accufatiuo cum, wo, quia illud regit, iomu : tei, fignificat modum extraordinarium of adnisationem caufantem: v. g. machicanuru tei uagoron jerarei, videat veftra dominatio modusm expectandi ideft quo modo expeChant. arifama, Jgnificat etiam modicm: v.g. me mo arerare nu arifama gia, ejt modus of figura qua nec projpicj poteft.

Sama, fignificat tempus quo fit adtio verbi cuipoltponitur, regitque cajum, quem verbum ex fe petit, poftjoszitur vero radicibus: v. g. faqe no nomi fama ni, quando actualiter bibebat vinum, iado ie caieri fama ni, quarsdo domum reucrtebatur, fùne jori ăgari fama ni , quando actualiter exibat enauigio, funè ni nori fama ni, quando astualiter confcendebat nauim.

Quando in oratione fuerint duo verba quarum actio fer modum unius fit, primum verbum debet efle in gerundio in do: $v$. g. mizzu uo morte coi, porta aquam, vel veni aquam portans, funè uo uoite coi, affer bic nauim, vel remis nauim trabindo veri, core uo totre ige, porta boc, wei tollendo boc vade.

Gerundium in, do, adiunctum verbis, rogandi, dandi, az!t gratiam faciendi fignificat rogare, vel petere rem lignificatam per. verba quibus anteponitur: $v . g$. nifon guchi uo uoxiiète cureio, doce me linguam I aponicam, fo uoxerarete cudafaruru na, ne dicat boc veftra dominatio, Deus no coto no catatte tamore, facias mibi gratiam referendi res que ad deum pertinent.

Particula, mo, pofpoftta gerundijs in, do, que in, te, vel, de, fisiuntur fgnificant, quamuis: $v . g$. fo moxite mo, quamuis boc dicas, fo iuaide mo, quamuis boc nor dicas, ica födo fufu méte mo, corobu mai, quantumcumque mibi perfuadeas; fidem non sbnegabo;etiam rituntur boc modo, fo moxéba attemo, etiarn $f i$ boc dicas, \& doxitemo co xitemo, quodcumqque facias drc.

Si particula, colo, de qua fupra, pofponatur geriendio in, do, affirmatiuo, o finiatur oratio in predicta particula fit oratio nergatiux: v.g. mice cofo, nalla tenus vidi, atte cofo, nut10 modo eft. Si vero oratio zon finitur in, colo, eftaffirmati- 
ua do emphatica: v.g. mite cofo gozare, vidi profecto. funitun verbum in, e, fecundum regulam fupra pofitam quando tgimus de particula, colo.

Quando verò gerundio in do negatiuo finito in, e, fubfequntur vel, ua, vel, naranu, aut, calla uanu, dicit nece/fitatem, of impoffibilitatem ad contrarium : v.g. mairaide cara uanu, eft neceffarium ire, iuaide uà nò còto narédnmo, nànto xô ca? of jifit res qua neceffario debet dici; quid faciam? xitãgauaide naranu , eft necelfarium obedire; eundem etiam fenfum, \& finon cum tanta vi, facit futurum infunitiui; tam afftrmatiuum: quam negatiuum, adiuncto tamen ilii fubiunciiuo fermiffiuo crm, dòmo:v.g. mairo còto de gozatta rẻdomò , quamuis ire debuiffem, mairu mài querẽdomo, quăuis non efem iturus, mairo còto de gozanacattarédomò, quamuis non debuiffemire. vtuntur etiam gerundio in do negatiuo ad figr ificanaum, $n \sqrt{2}:$ v.g. òracio uo mclaide cuna, ne comedas nifi prizs orationtm feceris

Gerundium verò finitum in, e, fignificat actionem iam effe, factam: v,g. mèxi cúte còi, venias poft trandium, còno qióga caité gozàru, bic liber eft foriftus, chichi ni fùmi uo cacaide cuiaxiugozaru, poenitet me non mifipe Epiftolam patri tuo, còno gióga caité gozaranu, non eft fcriptus bic liber.

Poftpofita particula, nagàra, radicibus verborum, quando Jibfequiturverbum fignificans actionem repugnantem aut aduerfatiuam, facitgerundium in, do:v.g. Tóganin Deus iòri bitcutài no go uon, ò uqetatc matçuri nagara ; caiette fomùi tatematçuru, peccatores recipiendo vel etiam $\sqrt{2}$ à Deo accipiant berrefitia maxima, loco gratitudinis; ipfum fotius offendunt, jefu Crifto Deus de gozari nagàrà , fito ni tàixitè cruzni càcaraxerareta, Iefus Cbriftus cü effet Deus crucifixus eft propter bominem.poftponatur etiam, nagàrà , nominibus: v. g. quantai nagard, quamuis fit inurbanitas, fannin nagara, tres fimul, vel otiam fifintres, aqiraca nagara, quamuis fit clarum, bic, aqiracana amittit, na, \& $f i c$ in adicetiuis qua finiuntur in na.

Poftpofita particula, iàfui, radicibus verborum tam actiucrurn; quam paliuorum facit illas fupinum in, tu, v.g. iòmi iafui, facile lectu, còrofare iafui, facilis occifu, adidem tendunt bi modi loguendi, iúte uà uò foròzij, eft dictu tremendum, 
mite ua fuxiguina, est res admirabilis vifu, iú no mò voloroxi, eft dictu iremendurn.

\section{$D E A D V E R$ I I $S$. Caput primum .}

F $X$ adiectivis finitis in, ai, funt aduerbia conuertendo, ai, H in, $0, \sigma . g$. fucó, profundé, ex finitis in oi, conuertendo illud in, $\hat{o}, v . g$. caxico, prudenter, ex finitis in, ei , conuertendo illud in, ế, $v . g$. xigueo, frequenter, exfinitis in, vi, conuertendo illud in, u, v.g. aiau, periculofe, ex finitis in, ij, conuertendo illudin, iu, v.g. cauaiu, mi Serabiliter.

Fiunt etiam alia aduerbia pofpofito, te, radicibus verborum v.g. fàdamète, determinate, vel probabiliter, aràvarete, marififle dor.

\section{De aduerbijs locorum}

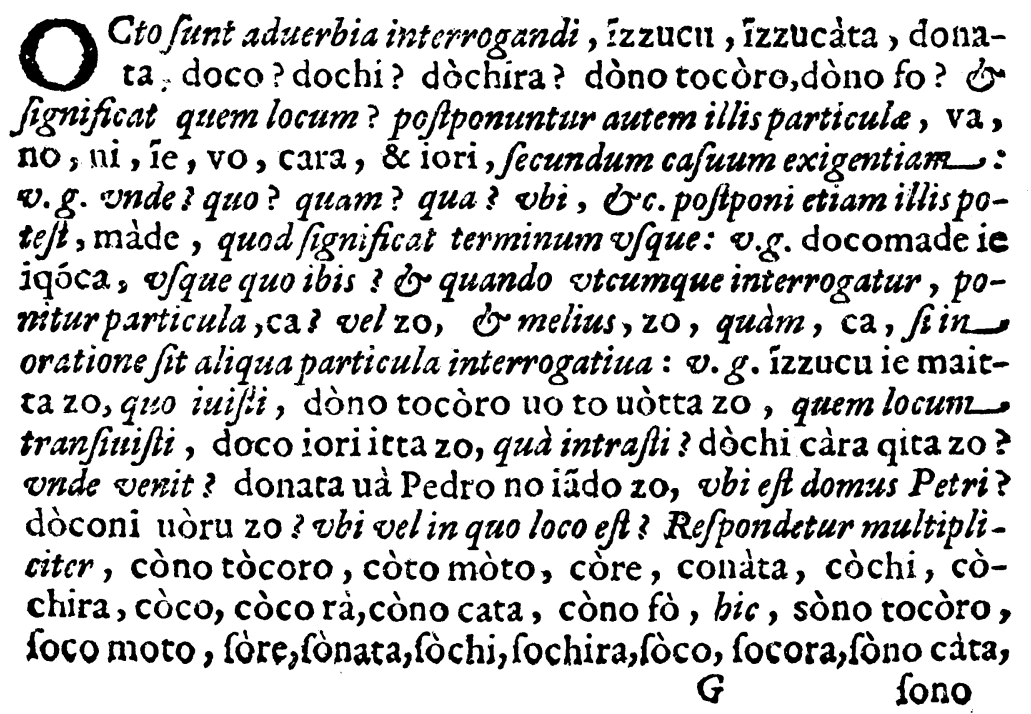


50

DE C O N I G

fono fo ific, ano tocòro, alo co mòto, are, anata, achi, àchira, alocò , àfocora, àno càta,ano fó, fignificat : illic, po/tponuntur bis aduerbys particula cafuales. Aduerbia interrog andi cum parti-ulis co, ialibas dv noftpofito, mo', Jignific ant vbicumque:v.g vel quacumque, vel quoczmque: v. g.. donotocòro ièmò : uoro,tranjibo quocumque, doconimo vibicumque, dòco cará mo, undequaque:.. Si verò loco, mo, pofponatur, nàri

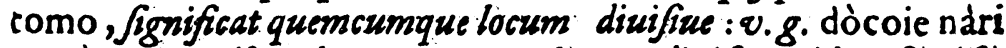
tomò mairo, ibo: ad quemcumque locum diuifue idem fignificat , doco zo, cum eifdemparticulis cafualibus of polfunt poni inter, doco, $\delta, z o, v . g$. dòco ni zo dru fodoni, fi quidem eft in aliquo loco, còco caxicò, fignificat bic ơ illic, doco mo caxicomo:, /gnificat omnem locum.particula cafuales: folent poni ante mo, v.g. do còni mo caxico ni mo, in omni laco', aduerbio veró anticcedenti poßtponunturv.g còco cáxico ni, bic to illic,, còco caxico ie dòco,caxico iori \&c.

Particula, vie, Jignificat Jupra; petit ante fe fuppo/itum in genitiuo: v. g. fàndai no uie ni uoge, pone fupra men fam, còno uie uà gozaru mai, non erit fupra boc : idest melius, fono vie ni , circa: ġfud, fòno, uie no fáta uo catàriare, narra qua circa boc funt, còre uaízzure jori mò uie de gozaru, non inuenictur; quid boc fuperiuss:idefi boc eft fupremum, xita, fignificat : infra: bi regit cofdem casus, quos pracedens : v. g. fandai no xítani uòge, pone fub menfa, micotóba no xita iori, quando rex: v. g. finiuit loqui, uoxita uo cudafarei, det mibi ueftra dominatio reliquias fui potus, que fuperauerunt.

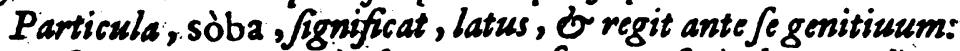
v:g. fito no. sòba uo fanaruru, feparat fe d latere alicuins.

Maie, fignificat ante: $\delta$ regit ante fe genitiun : v.g. fito no maie uo touoru, tranfeo coram alio, cacúgò no maie, $\int e-$ cundum difpofitionem, funbet no maie, ficut credo vel cogito, vel iuxta fenfum.

Mauari, fignificat circum circaingiro : Or regit ante fo genitiuum: $v \cdot g$. igléfia no ma uari ni tçuchi uo nãgue futçuru na, re proÿcias terram in circuitu Ecclefie.

Vchi, Jignificatintra, of Fiante fe babet fubflantiuum debet -ffo in genitiuo:v. g iglefia no uchi ,intra.Ecclefiam, ano fito na; fito no uchi de uanai, ille bomo non chf inter bomines, idefi 
non esf bomo, fútaciichi cúta còto ua, cúta uchi dellanai, co* mediffe duas buccellas, non eft comediffe.

Foca, fignificat foras, vel extra: \& petit ante fe gemitiuum: v. g. igletiano toca ni, extra Ecclefiam, focaie iqe, exi vel eas foras. aliquando loco particule genitiui, ponitur iori, v. g. Deus uonägo ichinin iori foca tçucuri tamaudıru, Deusnon creauit nifi onam mutierem; idef nallam formaust extra onam, Tèngu fito ni àcu uo fufumuru iòri fòca uà, nài, Da:non nibil facit nifi persuadere peccata bominibus, goxo uo tdfucàru tàme baptifmo vo lazzucàru iòri fòca bechi no michi ga nai, non eft alia via ad Saluandum bominem extra baptifmum; ideft, abfque baptijmo nemo pote/ Jaluari, Deus no gracia iori foca, abjque deigratia.

Naca, fignificat medium in quantitatibus continuis vel diforstis v.g. qinò náca ni, in medio ligni, fito no nàcaui, in mediobominum.

Nacaba, Jignificat medium in rebus Jucce/finis : o vtrumque petit ante fe genitiuum: v. g. dangui no näcaba ni, in medio fermonis, fòre uo qijte, nacabà uà uofore; nacàba ua aqirete ita audiens iffud, \& metuit \&r expauit, idef medium tempus confumpfit.timendo or.

Ato , fignificat retro, of regit ante fe genitiuum: v. g. Ionata no àto cara mairo, veniam poft te, ideft, te fequar.

Vàqi, /grnificat latus : et petit.ante fs genitiusum: v.g. Pedro no uaqi, ad latus Petri, mila no uaqi, finita mi $\int a$, cono uaqi bis diebus prateritis, omniz pradicta aduerbia requirunt poft fo casfus quos petucnt verba quibus jubfequantur.

\section{Aduerbia ad caufam interrogandum \& ref- pondendum.}

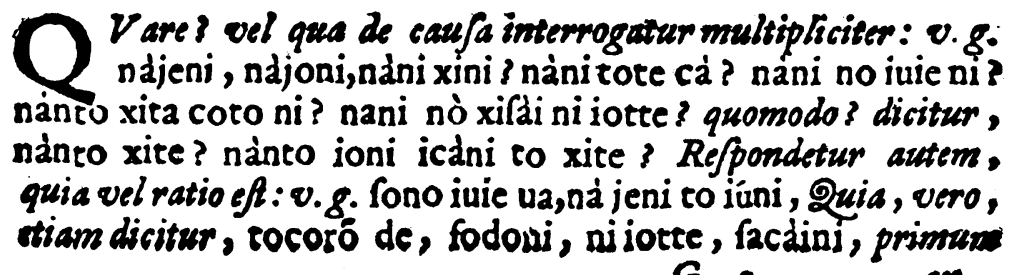




\section{2 \\ D A D V E R B I I S}

ex bis tribus dicit multam carefalitatem, fecundum verò non tamtam: tertium autem minorem .

Vie va, Jignificat: cum vel fiquidem: v. g. tóganai vie ua ci zzucai ga nai, non timeo, quia, vel faquidem non kabeo culpain, eunders fenfum facit particula, cara, v.g. caiōni irò vo misùru càra va; càcufu còto va irànu, iñ vanurri illud abfcondere tentas, fiquidem talem colorem oftendifli. S'i quidem, quafi illatiuum fagnificat, tòqi va, \& , xicaru tòqi va . fari na gard, , $\sqrt{2}-$ gnifieat, Jed; sàri tòte va, fignificat, cadbuc: sàru tòte ua, $\sqrt{\mathfrak{L}-}$ gnificat, fiquidem resita fe babet: sàrú tòte ua, qicoienu còto

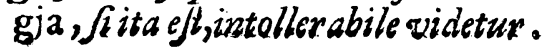

\section{Aduerbia temporis .}

$\mathrm{Q}^{\nu}$ $V$ ando interrogatur per, itçu, vel, itçügoro, a quot diebus? interrogatur per, icca sàqi, vel, icca maie, a quot menfibzus? interrogatur per, icutçuqi faqi ? aquot annis interrogatur per nànnèn maic, folet aldi, ni, quando verbum illud petit, \& Semper in fine interrogavionis ponituer, $\mathrm{ca}, \boldsymbol{v} c h, \mathrm{zo}$, \&o melius, zo?

Respondetur: nunc, ima, vel, tăda ima, iam, mo, v.g. moiqe, easiarn, aliquoties dicitur, tògi ni iotte, vel, jibùn ni jotte, postea dicitur, nochi, sòre cara, wel, sòre iòri, post ifud, còre càra, vel, còre iòri, poft boc, àre iòri, vel, àre càra, pof illud. Statim dicitur, iẫgate, poftea, vel deinceps dicitur, ima iòri nốchi, vel, ima iori xite ud, vel, ima iorì igo, vel, jïgònigo .ifto mane, dicitur qífa. connichi, vel, qio bodie, áfu, vel, miônichi, cras, cras manì afá, àxitatô, vel', àfatòcu, crasnocze, miònia antea, ijen, vel faqini, beri qinô, vel, sàcujit, nudaius tertius, uototoi,vel fùtçuca sàqai $\mathrm{ni}$, diebuspretteritis còno gị , còno fōdo, vel, xenjît, vice preterita. 1 dem xendo. vice futura, còndo, ab binc duobus diebus, asàtte, vel miögo nichı, poft tres dies, xiasàtte, vel miơ mió gonichi, qiônen, vel, cơzo, anno praterito, bac anno, còto $x i$, atribus iam annis, vòto tòxi, vel, uotódoxi, $a b$ binc vero quatuor annis. fanuruwotódoxi ftatim, tàchi màqi 
vel, focuii ni, funauachi, ftatim profecto, tàntegi, in illo momento.

Itçumade? afque quando? itçu maderno ; femper, itçu càra a quo tempore, itçu iori, ex quo tempore?

\section{Aduerbia negandi .}

Tia, vel, iia, fgnificat non, fó deuanai, non ef ita, iccana vel, iccanágueni, nullatenus, iùme iùme, neque per fomsium, fàrani, vel, ichiien, càrçutè, vel, càtçute mòtte, nullo modo, iò, iòmo, vel, iòmo iòmo, neque cogitatione: v. g. càtçute màiru mài, nullatenus ibo, iòmo fó uà gozàru mài neque in mentem venit quod ita erit; \& quando predicta iunguintur affirmatiuis faciunt etiam fenfum negatiuum, v.g. iomo iòmo to moxitaréba uo màiri atta, cum dixiffes tenon. iturum: iujfititamen, io mairó, nullo modo ibo.

\section{Aduerbia affirmandi .}

NT Acanàca, fggnificat: ita eff, uó, fignificat: fac, alfentienNo, gueni, vel, gueni gueni, ficfe babet: v.g. guèni guèni fó no aró, abfque dubio fic Je babebit res, chōdo, Jignificat, omnino, laiòni, sòno bùn, sòno gotòqu, fo de gozaru, fò re fóre, màffo gia, xicato, fignificat : ita efl, mòttomò , $/ 2-$ gnificat rationabiliter loquitur, guioi nó gotoqu, ficut veflra credit vel dicit dominatio, mòchi ron, non cadit Jub dubio feu difputatione, nàcanàca nàru còto de gozaranu, vtrè non est poffibile, nàcanàca no còto, res eft cui poteft afjentirifou fides adbiberi, ma cùtoni, verè, \&े idem fignificat, xinjt , vel, xinjit $\mathrm{ni}$, xei mon, iuramento confirmo, i asàca, vel, ifasàca mòtte nec parum quidem, iffai, vel jxxet nulla via, nullo modo, \& quando iunguntur affirmatiuis Jgniffant prorfus in omni euentus. 


\section{Aduerbia comparatiua.}

I

Ori, iòri mò, iòri mo nd uo, fl'gnificant magis comparatiuè, perfona qua comparatur eft in Nominatiuo; cui comparatur vero in ablatino cum aliqua ex pradictis particulis : v.g. Pedro ua juan iòri mò gàcuxó de gozaru, Petrus eft doctior Ioanne sòcoie nobòru iơri ua ; mairanügà màxi gia, melius eft non ire; quam afcendere iflbuc . gotòqu, mama, ioni , fiunt aduerbia Imilitudinis, of patunt ante fe genitinum rei cui fit a/fimilatio: v.g. Pedro nó gotóqu, Jicut Petrus. Si veró antecedit verbum, non petunt genitiuum; v. g. no iama le nari tomò qi tài màmani gite, nururéba, nügui fuceraruru, etiam fi eant ad campum, or montes fe indurent, $\sqrt{2}$ wolunt tali vefe, or illam exuunt quado aqua madefit, uomô màma ni, vel, uomô gotoqu vel, uomôi ioni, fucut cogito, conòmi no màma ni , fecundum defiderium or ad eizus menfuram, födo, fignificat, tantum, vel quafi; $\delta$ proportionem: $v . g$. qifén ano fito fódo no gacuxó de gozaru, tam doctus es ficut ille, faräga cüdàru fòdo iòi, in tantum erit fanus in quantzom babuerit ventris purgationem, míchi uò arùqu fódo cutabiruru, $f(6)$ t ambulo, fic deficio, acai fódo ioi, dum est rubicundius, tanto melius, xinuru fódo no vazzurai de uanai, infirmitas bac non $e f l$ ad mortem idef mortis caufatiua, funè ni mefaruru fódo narăba uare mo norǒzu,$\sqrt{2}$ veftre dorninatio tantum vult af fumere laborem vit nauim of cendat ; ego etiam, taméxi mo nai fódo ni atta to mo fu, dicunt fuif$\sqrt{e}$ ficut nunquam, uoqùru fodo aräba fòre ie mairozu, $\sqrt{2}$ ad Hatum perueniam quod po/fim i leEto furgere conferam me advos, chicara no födo uo mite, videns virium proportionem födo nǒ

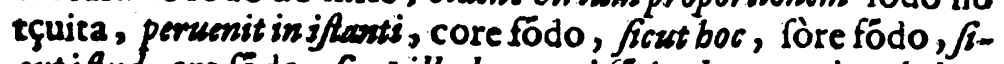
out iftud, are födo, sacut illud, uouoi födo dum magis vel plus, fucunaifódo, dum minuss. 


\section{Aduerbia fuperlatiua.}

$\mathbf{V}$ Ie, fignificat rem Jupremam : v. \&: chriftianno voxiie ua izzure iori mo vie de gozaru, doctrina o fides Cbrifiana est fuprema of Juper omnes, còno faqe no vie ua nai, non eft melius vinum ifto, ichi : vel, daiichi, ef fupremum or enicum: v.g. gacuxo no uchi ni Sancto Thomas daiichi de gòzatta, inter doctores Sanftus T'bemas ef fupremus, còre ua are iori vie, boc est fuperius illo - particula xita, eft contraria, uie, Jignificat vexó inferius: $v . g$ xigitai ua anima iori xita de gozaru, corpus efinferius anima.

\section{Aduerbia intenfionis \& exaggeratio nis.}

I

Chidan, chicägoro, icco, fignificant valde: v.g. chicägoI ro no uo cocoró gaqe de gozaru, ef maxima cura \& diligentia, b c. före ua icea uarui còto gia, iflud eft valde malum, bexxite, Jggnificat precipue, tòri nage, figniffcat in particulari vel Specialiter, coto no foca, raro, \& extraordinarie, icanimo, fignificat, valde, amari ni, fignificat nimis, \& denique ex adiectiuis formantur aduerbia modo supra dicto, que $\int \mathrm{g} g$ nificant aduerbialiter quod adiectiva ab folute: $v$.g.ex fucais quod eft, profundus, a , um, fuco , quòd eft profunde, icani mo xizzucani vaide quiete, tani coto ni, extraordinarie, xitàtacani, vel, guiö fanni, formidabiliter idef, nimis. vide in dictionario.

\section{Aduerbia congregandi.}

TT Oxinabete / fgnificat oniuerfaliter, fobet, communiter, idem J Ignificant, tçune ni, vel, lojite, feijeini, regulari-

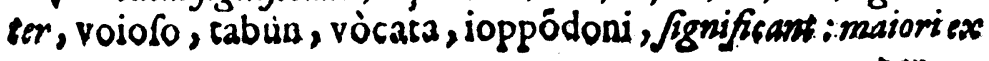


parte, qèccu, vel, caictte, Iggnificant, quinpotius, vel, tènnèn, fignificat forfan, \& idem fignificant, xijen, icafama. Fadametc, fignificät probabiliter, canarazu, abfque dubio indefeEibiliter, moxi xjien, fiforte, xotocu, naturaliter, jinen, cafu, xïdai xídai ni, vel, jèn jenni, vel, xidai ni, fucce/füue lenter, vonozzu cara, per fe abfque alio.

\section{Aducrbia concludendi, \& aduer- tendi .}

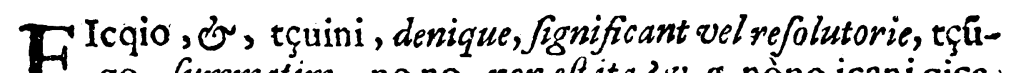
If go, fummatim, no no, non eft ita ? v. g. nòno icani qicaxeraruru ca? beus audis ne? moxi, fignificat etiam; beus; Jed ef vocoúulum orbanum : v. g. moxi Padre fama, beus reserende pater, iare, efl etiam beus, cum inferioribus: $v . g$. iare taro quaja to iièba dicens beus, taro quaja. iaifignificat etiam beus fed vilius: v.g. iai fochi ga mòtta mono ua nanizo? beustu quid eft quod portas? idem fignificat, ia, v.g. ia uo tòno bara domo ùa nani uo fauãgu zo ? beus vos milites o bonibomines quid turbamini? ad eundem fenfum tendit particula ai, $p a$ fpofita v. o. izzure mo mina iô qiqe ai, beus vos omnes audite.

Particula, ca, $d$, zo, ot fupra dictum eft, funt ad interrogandum. idem babent munusparticule ia, vel caia, fed Junt bumiliores: $v . g$. are ua tare caia ? quiseft ille? còre ia, iftuds io fuqc̀te tare ca ua tazzuneô zo? cum fit alìa nox, quis poteft interrogare, \& querere? sòre de aro ca toíu còto gia, dico, $\sqrt{b}$ erit iftud?

No? petit confenfum:v.g. gozaro ca no ? veniet? nonne,? mairo to unxerareta no? non ne dixit quod veniet? no Pedro dono? non eft ita domine Petre? na, fignificat idem ; fed eft inferius: v.g. foqiita na, nonne $\int i c$ audifit 3 aliquando in oratione vbi eft zo, folet poni ; baxi; que eft particula dubitandi : $v$. 8. nanto xita xifai de baxi gozaru zo? qua de caufa accidit boc ? fatc nanto iu uogiacu de baxi gozaru zo, quomodo vocatur vefter ifte bofpes 3 goiô baxí gozaruca ? eft ne tibi aliquid neceffarium ?

10, vel, zo; affirmant of faciunt cadentiam orationis: v.g. 


\section{DE PRAEPOSITIONIBVS}

caica zo, vere fcripfit, maitta io, venit profeczo, fono tò qi vare va ichi dan varuı tçucai vo xiraruite gozaru io, tunc profeCto mala nuntia feu miffones fum exequtus of feci, bacari, $\sqrt{2}-$ gnificat, tantum vel folummodo, sóre ni cãguitte, istud folum, còre ni căguirazu, non boc folùm. aliquando, bacari, fignificat magis velminus: v. g. fiacu bacari, erunt centum, fiacunin bacari corofareta, centum vfquebomines occififunt, nó, nóte, naqu xitc, naqute, fignificant fine vel ab/que : v. g. raxximo ná, abfque ratione, $\mathcal{O}$ ordine, cacügò nó, abfque praparativne.

Aäuerbia fonus funt mulitiplicia fecundum diuserfitatem quam Iapons in fonus terminatione percipiunt, $\mathcal{\delta}$ illis, to , folent poAponere: v.g. ua ua to xite, vociferando dicentes, ua ua, $\mathbb{E} / i$ illis pofponitur meqi, u, fignificat talem frepitum facere : v.g. ua meq $i, u$, va dicendo vociferor, aris, ¿ $\sigma c$ 。

\section{De Prepofitionibus cafuum .}

T Ame. Jignificat ni vel erga: v.g. fonata no tame, tibi, 1 regit ante fe genitiuum, nanno tame, ad quid, nanini naru ca? ad quid cft? nani ni xô ca? ad quid faciendum babes illud? nani no iô ni tatçu ca? ad quid eft receffarium vel proficuum? maitte no iô ua? qua ne neceffitas efl eundi?

'Tai xite, Jignificat propter, vel contra, v.g. tègu ni tai xite te gito, pugnare contra damonem o ei refiftere, Deus ni tai xite cúguio no coraiuru, Juffero labores propter Deum, uie iori, Jignificat etiam, propter: w.g. uon jif no uie iori, propter fuara mifericordiams.

Ni iotie, fignificat etiam caufam quare: $v . g$. Deus iori fito no iento acu ni iotte go fempô uo ataie faxerareozu, deus tribut bomiribus fecundum opera corum vel vitÿ vel virtutis, deriuatur a verbo, iori, $\mathrm{n}$.

Nitçuite, figrificat circa, \& deriuatur a verbo, tçuqi, $u$, v. g. còre ni tçuite, vel còre ni tçuqi, vel, còre ni tçuquete, circa boe, fono gui ni unite ua zonjenu, nibil circa boc negotium fcio, vôxe uamottomo narédomo uágami ni totte ua canai gatai, beme disit veftradominatio; Jed quod ad me attinet efl 


\section{8 DE PR AEPOSITIONIBVS}

factu difficile, Dai guan ni itatte ua ichinin bacari fadameio, quod ad economum feus maiordomum attinet, vnum tantum. confitue, bac omnia, itatte, v.g. tòtte, funt gerundia verborum ficut of pracedentia, etiam folent dicere, Hadre còto ua quod ad patrein attinet, uarera còto ua, circa meas res, vel quod ad me attinet, xităgatte, vel, xitägote, figniffcătiuxta, \&. funt gexuscdia verborum, xitãgari : u, \& , xitâgai: ó, onde ante fe regunt datiuun $\int$ icut corum verba: $v$. g. guioi ni xitägatte, vel xitāgote, fecundum quod vestra pracipit dominatio, xidai fignificat idem: v.g. conata xidai, ficut voluseris, aliquando etiam iungitur radicibus verborum: v. g. mxiri xidai, fecundum quod venerit vel eius aduentum.

$\mathrm{Ni}$, fignificat locum in quo.idem fignificat, ni voite, fedpermanentius: v.g. go fatto ua fuximi ni uoite uôxeídafareta, banc legem pofuit dum eljet in ciuitate, fuximi Bungo funaini itatte, in ciwitate Funairegni de Bungo: iglefia ni uoru, eft in Ecclefia.

De, fignificat locum in quo fit actio: v.g. michide Pedro ni uota, obuium babui Petrum in via;eadem particula, de, or uomotse, fignificant inflrumentum quo fit actio, bo uomotte Pedro uo uchi coròita, ligno Petrum cecidit, Padre fama catararetäde nano qicoieta, ex quo pater rouerendus illud narrauit melius fuit intellectum., necqi de xinda, mortuses of $f_{f-}$ bri.

Cara, vel iori, fignificat locum e quo vel onde: $v . g$. iglefia cara , ex Ecclefiasetiam dicunt, fune cara maitta, nauigio venit, cachi cara maitta, pedes venit, funé de maitta, eft idem quod, fune cara maitta, of fune ninotte maitta. fana cara me cara miguruxij mòno gia, eflindecorus oculis \& naribus, iori, $\sqrt{1}-$ gnifuat locum per quem: v. \&. Lama iori faitta, intrauit per fenejtrame.

Tomoni, fignificat ; fimul: v. g. fonata to tomoni mairozu ibo frnul tecum, mofu to tomon, ifimul ac dixit.

Ie , fignificat locum ad quem: v.g. achiie marro, ibo illue, mi aco ni faite mairo, vel miiaco ni muqete mairo, ibo directe ad curiam, miiaco no cataie noboru, afcendit ad curiam idem, miiaco no foie noboru, aliqui dicunt, miiaco no iori, vel miiaco fana, vel miiaco no gotoqu noboru, fed non eft bonus loquendi modus; fed rufticorum - 
De, fignificat materiam ex qua: v.g. tçuchí de ca uaira uo tçuquru, regulas ex terra vel limo conftruere, nànde còre uo tçuqùru ca? ex quo boc facis?

Vie, fagnificat erga: v.g. zuỉbun códomo no vie uo fito ni mo ndxi marafuru ioni to cocòró gaqe marafuru ; amni cum diligentia curo circa vel erga vel de meis filys, quomodo illosfaciam bornines.

Sònata no fiquánno vo Gaiban mefarèio, babeto curam detuis Seruis?

Made, Jignifrcat ofque: $v . g$. àfumade, vque mane, inòchi vo vxino made aru mai, non ammittet vitam vel vfque ad vite amifionem non perueniet, fore made vomoi mo ioranu gui gia, non peruenit ad mentem meane $v \int q u e ~ a d b u c$, cocòro zaxi areéba canauanumàde mo xei uo iruru, quando aliquid defideratur, adbibentur vires vfque ad impo/Jibile, molu made mo nai, non eft neceffarium dicere, còno tocòro made maitta, bucufque weni.

\section{De coniunctionibus \& diuifio- nibus.}

70 , figuificat, of , copulatize: $v . g$. Pedro toj uanto $\mathrm{Na}$ 1 gafagi ie ita, Petrus \& Ioannes ierunt Nagafaquim, còreto, àreto uò tòru, accipio boc ơ illud. idem fignificat, mo, $v g$. Pedro mo juan mo Nägafaqi càra módotta, Petrus or Ioannes redierunte Nagafaqui,naqu mono mo ari , uaró mòno mo aru, funt qui flent, of funt etiam qui rident, mo, anteponitur multoties negatiuis : v.g. nanigoto mo gozaranu ca? non ef aliquid noui?

Mata, fignificat, \&, vbicumque inueniatur fiue inter nomina fiue verba, ca, Jignificat vel: v.g. Pedro ca; juan ca coi to iie, dic quod veniat Petrus vel Ioannes, arui ua, fignificat etiam vel: v g. arui ua Pedro, arui ua juan, vel Petrus, oel Ioannes, arui ua iomu, arui uamono uo caqu, vel lego vel fcribo, moxi ua, fignificat $f i$ vero in medio orationis: v. $g$. móxi ua cdnéga nai naraba, oc. Jivero non babeas argentum.

Ad orationum contexturam vtwentur, mata ua, eb prater boc

$H_{2}$ vel 
60

DE INTERIECTIONE

vel praterea : v.g. arui ua iamaíga uocoru ca, mata ua ifögui no fumi qita ruca \&c. vel accidit aliqua infirmitas, preter boc. fi verit aliqua epistola.

Xicaréba, fignificat: cum res ita fe babeant, fari nagara, fed, fo aru tocude, cumboc ita fit, faraba, cumboc ita fe babeat, farëba fareba, cum ergo doc.ca? Jgrnificat /2? v: g. maitta camiio, vide fi venit vel iuit, maitta ca xiranu, nefcro fi venit, iara, fignificat fi diuifiue: v.g. fito iara chicuxo iara xiranu, nefcio vtrum fit bomo véch animal, nani iara to moxita, nefcio quod dixerit.

Exparticulis, nanica, o tòcàcu, intromitfis alÿs particulis fiunt quedam quaji difiunctiusa Seu exageratius: v. g. nani ia ca ia ? qua res? idem est, nani iara ca iara ? or nànto iara cato iara? nanto xite, ca toxitc, quomoda? nanta mo ca tòmo, nullo modo, nanimo camo, nibil, ranigoto mo cägotomo, mina içtuuari naruzo, denique omnia funt mendacia, nanino cano vel nanto, xite cato xite, modus excufandife, nanino cana to iúte, dicens boc \& illud, domo como, quomodocumque fit idem, doxitema co xitema doxite ùo xite, faciendo diuerfa boc đo illud, do xôco xồ, faciam boc ơ illud.

Tòmo cacumo, in omnibus, idem, toni cacuni, idem etiam tonimo cacuni mo, vel totema cacutemo, còre to if; care to ij, boc of illud dicens: ideft excufationes, care core, illud or boc coco caxico, bic of illuc, conata canata, ific \& illic, uomo xirố, uocaxu, accammodando fe quafi adulari.

Siparticula, mòtte pofpponatur particulis, catçute, mate, ifafaca, tomoni, nani, nani nani iori, adiungitur illis efficasia f-energia: v.g. catçute motte fo aru mai ${ }_{2}$ nullo modo erit talis res.

\section{De interiectione.}

S Ate, fatefate, fatemo fatemo, funt interiections admiran$\mathcal{H}$ tis: v. g. fatemo. Deus no uôqinaru uonjificana, a magna mifericordia Dei!

Auare, eft interiectia miferentis: $v . g$. auare mutçucaxij iò no naca cana, o mondus repletus miferÿs. 
$H . x ! e^{\prime}$ interiectio panitentis: v.g. Ha fuxi demo uomoxiroíga; tocoro niiote qi coie canuru, o lebor fonus \& cantus barmonis eft valde gracilis; fed non bene omnia percipiuntur!

Iara! eft interiectio tam latitio ; quam triftitia: v. g. iara iara medetaia, o quantum gaudeo ơc. idem, ia, v. g. fatemo iiaxii iatçübaraia ! o quam vilis \& abiectus! gongo dodan fuxiguina xifai cana! o quam rara of ridicula ratio, iei, eft intcriectio mirantis: v.g. iei Padre fama cochi gozaru io, bic est pater!

Hat , est interiectio eius qui repente terreiur: $v . g$. Benqei co-re uo mite hat coto naxi to zonjite, iono màma niuani bo uo voroxi \&c. videns boc benqusi is.

\section{De fintaxi, \& carbus, ginos regunt verb: :}

NOminatiuum ponitur in principio orationis, verbum. 1 vero in fine, reliqua vero fecundum cadentiam: $v \mathrm{~g}$. Pedro ua Nagafaqi de xutrai xita iqi iqi nitçuite juan uo coroita, Petrus occidit Ioannem circa vel in quadam differentia que fuit vel accidit Nagafaqui: in aliquibus orationibus grauibus verbi fuppofitum ip $\sqrt{3}$ poftponitur; fed raro:v. g. tare mo cana uanu futari noqimi ni tçucoru còto ua, nemo potest duo. bus dominis feruire bic, eçucoru coto ua, eft verbi fuppofttum, core niiote tanomi tasematçuru itçumo uirgen, ideo pracor Beatam Mariam Semper Virgitem.

Orationes fubiundiui abfoluti vel permiffiui, of infinitiui, or canditionales, of caufales femper anteponuntur orationibus de indicatiuo aut imperatiuo; etiam $\sqrt{2}$ in linguis Latina aut Eu. ropais non fiat fic fenfus : v. g. achi cara tomeraruru tomo; tomaru na, ne remaneas etiam $f i$ illine detinere te velint, , sòre uo qiitarêba, fara uotacete módotta; cum bec audiffet, iratus réserfus efa, taxicani uqetamotta niiotre colo, móxi ásne maraxitare, ftceliter audiui, ideo retuli \& dixi, Eaio gozatta raba uo mexi uo xinjô mono uo, fi cito veniffes dediffem tibi manoducare.

Quando Junt duo verba siufdem temporis in sadem oratione., 
primum erit in gerundio in, $\mathrm{e}, \mathcal{S}$ alterum in tempore quod orationis fenfus exiget: v.g. còre uo tòtte giqini miga como no ni uataxe, accipe boc o trade feruitori meo.

Quando vero fuerint due vel plures orationes qua loquntur de codem fubiecto vel tempore, folum vltimum verbum erit in tempore, quod orationis fensus petierit, reliquorum vero aliqua erust in radicibus: alia autem in gerundio in, e, $v . g$. touazunba cotaiezu, voxe aräba tçuxxinde qiqe, $\sqrt{2}$ te non interrogant ne refpondeas, $\sqrt{2}$ loquantur audias attente, Deus no no còto uo macotoni uqe, go uoqite mo cama uaide, fono mama inferno ni uochita, neque in Deum credidit, neque eius mandata Seruauit: vnde cecidit in infernum.

$V$ erbum Semper fequitur in bonore tertiam perfonam;nullus enim fe bonorat ni/s fit rex qui de fe loquens dicit, iorocōbi ni uóboximefu, gaudeo plane.

Quando fuerint multa adiectiua omnia erunt aduerbialiter prater vltimum: $v$.g. qe nango, iro curô, icanimo vtçucuxij mono, erat Jpeciofus valde, babens pilos longos \& colorem nigrum ofc.

Antepofita particula, to, verbis fignificantibus intelligere, credere, audire ort. fupplet verbune fumes fui, \& fignificat: quod v. g. fito tozonjita, crodidi vel putaui quod effet bomo, qixú uo jennin to vomo û́ ca\} credam te effe fanctum? amata po fito xini no fonouo ni moiuru uo mifaxerareô, videbis multos bomines ira flammis ardentes. bic Suppletur particula to , a moiuru uo guod efl verbi fuppofitum.quando verò pofponunt, mo, ad to, tunc tenaciter affirmant quod dicunt: $v . g$. mairó tomo, omnino ibo vel iturus fum.

Particula, to , in primo fensu fuppletur aliquando per, ioni, v. g. ägueta joni gozaru, dicunt quod obtulit, ica iona fito to ua xiranu, me fcio quis bomo fit ille. aliquando verbum fubstantiurum frupplet particulam, to v.g. mairó de gozatta, dixit quod veniet, xô de ua naquerédomo, quamuis non dico qüod faciam bc.

Qiuzo còre uo mite, ima uo fägo no coto dearéba, videns boc, quiuzo, credensque boram mortis iam adefle, bic verbum Subflantiuum fupplet, to \& fit quafi verbum actiusum regens accufatiuum, ima uo, fupplet etiam, to . particula, fona o guena fignificant, videtur, Cona, poftponitur radicibus verborum : 
v. g. dèqi fona, videtur quod finietur, $\sqrt{2}$ verò illi postponatur aliquodverbum fubftantiuum mutat, $a$, in, i,v. g. dègifonigozaru videtur quod perficietur, deqi foni mo zonjenu, credo quod non finietur.po/tponitur etiam, fona, adiectiuis finitis in, i, o illusd amittunt : v.g. io lona, videtur bonus vel quod fit bonus, xiguè fona, videtur frequens, a iau fona, videtur periculo fum, $\sqrt{2}$ veropostponatur adiectiuis finitis in, na, illud amittunt, $v$. g. aqi raca fona, videtur quod fit clarum.

Particula, guèna, polponitur nominibus \& verbisiam formatis : !v.g. maittã guena, credo quod venit $f$ autem illi pofponatur verbum fubflantiusem, mutat, a, in, i, v.g. maittã gueni gozaru, credo quod venit, fona, fignificat, videtur, or, gue$\mathrm{na}$, credo; fed vtraque vtuntur in occafionibus in excmplis expreffis.

Quando in oratione fuerint duo praterita, primum erit in tali voce; fecundum vero in voce futuri: v.g. qefa càra fo uôxerareta raba mo faia de maraxô, $\sqrt{2}$ boc dixiffe ifto mane; iam receffiffem.

Quando refertur id quod alius dixit, dicitur boc modo, Padre mósàruru ua : iăgate fonatà̀e mairó to mofaruru, Pater dixit fe ftatim buc venturum, aliquando quando fe excufant, folet fup pleri, to, per no, v. g. afu no, raigudt no, nàndo to noburu na ne differas dicendo quod cras; vel menfe venturo \&c.

2uands poft, $n$, fequitur, wo, amittitur, $u, v . g$. go uono uqe tatema tçutta, benefitia accepi.

Aduerbia femper anteponuntur verbis ; prater aduerbia temporis, que in principio orationis conftituuntur: v. g. fore uo qijte iccó xicàri maràxita, audiens istzod iwatus est valde, qiô nen efpana carà uatàttà tòqi, quanda anzo preterito ex Hippania. transfretaui. omne verbum requirit ante fe nominatiuum expreffum aut fub intellectum: v. g. uare iqe, uel, iqe, in quo fubir. telligitur, uare, vade, in aliquibus autem orationibus uidetur bac regula deficere: u.g. x: fai uóba core ni uà mósàre maràxo$\mathrm{zu}$, bic carefam of rationem refert feu dabit.bic nullusm uidetur nominatiutom, quin potius, are ni ua, eft datizum aut ablatiuum, dre ni uà, nauo uoixri atta, ille melius fcit.in ifla etiam oratione, dire ni ua, deberet effe nominatiuum, cacàru no ni ud eóganeno cufari uo icufugi mo tcuqeta dōg!u de gozàru, pro torque babebat catenam aureakn maltos babentem anulos. 
64 D E S Y N T A X I

Core ni ua gozonji arn mài, non cogno/cet de boc ueftra dominatio, bic, core ni ua, widetur fupplere accufatiuum quod regit , zonji : uru.

Verbum imperfonale aut infinitum petit ante fe nominatiuum : u.g. Pedro ua màtta to mófu, dicunt quod Pctrus uenit .

$V$ erbum, iri : u, pro indigeo, es, regit duo nominatius, rei, ô perfone indigentis: $u . g$. uatacuxi uà còno cánëga iru, ego indigeo uel babeo neceffitatem buius argenti etiam regit datiuum de perfona: u.g. fono tame ni uz cànega iranu, non indiget argento feu nummis.

Verbum actiuum petit ante fe accufatiunm pro fuppofito: u.g. càne uo motànu, non babeo pecunias.

Aliqua nomina, cobita feu coie, qua ut fupra dictum eft, funt médicata a lingua Cbinenfi regunt eofdem cafus quos verba I aporica que illis correfpondent; v.g. ni ua qenbut noaidani mèxi uo coxiraie io, prapara cibum dum bortum videmus, bic nomen qenbut, regit accufatiuum, ni ua uo, quia, $\mathrm{mi}: \mathrm{ru}$, quod eft video, es, regit accufatiuum, fito ni guenzan furu, eft idem quod fito ni uó, obuium video bominem, \& regit, genzan, datiuum ficut, $2 \mathrm{i}, 0$ :

Quando vocabulum cobitum ex duobus componitur, cognofcetur effe verbum, $\sqrt{2}$ primum, verbifignificationem babeat: $v \cdot g$.

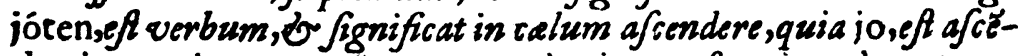
do, is, tenjo, vero eft nomen, quia, jo, pofponitur \& anteponitur, ten, quod jignificat calum.

Verba paffiua petunt ablatiuum pro perfona agente: v.g. Pedro carà còròsdreta, occifus eft a Petro, fed melius regunt datiuum: v.g. Pedro ni corofareta. Pedro ua nufu bito ni càne uo torareta, latrones furati funt pecunias Petri .

Sunt etiam aliqua neutra, que accufatiuum regunt ficut actiua: v. g. xiqitai uo fanaruru, difcedere a corpore, feu mori axi uo uäzzuró, dolere pedes, \&, nigue uru, pro eo quod eft fugio, is, nogàre, uru, euado, is : de, uru, exeo, is : nòqe, uru,recedo, is : touor $\mathrm{i}, \mathrm{u}$ : tranfeo, is: nori, $u$, nauigo, as, v. g. caixo uo noru, nauigomare, iugi : $u$, ambulo, as, michi uo iuqu, viam ambulo, uo uari,: u finior, ir is, mairi : u xǒgui uo mairu ad tabulam latrüculariam ludo, iorocobi, ui cocoro uo iorocòbu. latifico cor: abi, uru, mizzu uo abiru, laso me aqua vel aquam fupra infundo, audremi, u, mifereor, eris, canàximi, $u$, trifor, 
D E S Y N T A X I

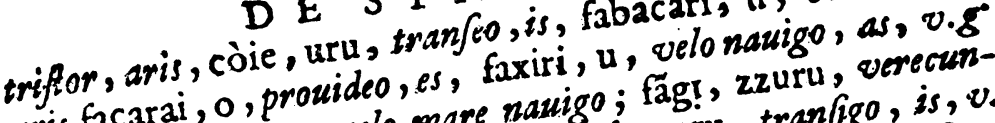
aris,facarai, 0 , prouideo, es, fare nauigo; fäg!, zzuru, verecun-

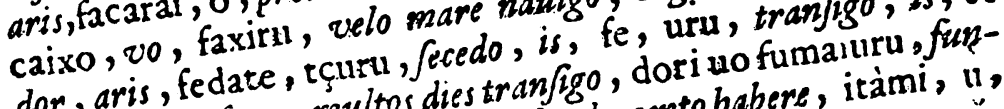
dor, aris, fe furu, multos dies tranfigo, dor faz bere, itàmi, $"$, g. Eacazil in ratione vel rationem pro fundamento cò to vàri uo mödoleo, es, ma vari, u, circuo, is: 0.8 . cixit nuntium, bic o $i b i$. xi mauattà, multoties ê per circul, $u$, năgufami, $\mathfrak{u}$, recreo, as, idem fignificat or regit, megurat cor latifico, as, naqi , u, ploro, as, tacocoro vo nagu, as, inochi vo tafucaru, pericu tuchi tçu, resedo do, goxo vo tafu caru, faluari in ucum vel a loco recedo, tomuis , v.g. tocòro vo tatça, de facio, is, vcăgai, ó, dubitando inquirai, ó, defunct is exeqfimo, as, vrìmi, u, quaror mè vò xinòbu, ro, occulte expecto quafi infidiefe: Q. S. Cictis qua requirunt accua, occulte fiquis me videt, aliqua ex pradictis qum particulis, cara, fatiuum loci, admittunt etiam ablat eft idem quod, tocòro iori ta-,

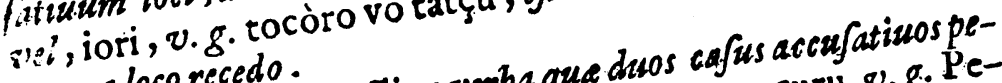
içu, a loco recedo.

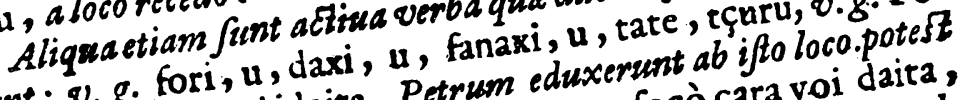
tunt: v. $g$. fori, vo voi idaita, Petrum eduxerunt focò cara voi daita, dro vo foco vo latiuum loci $: v . g$. Pedro vo loco $\mathrm{g}$. fito vo, vel, etiam regere ablat vatiuum vel accufatiulur, Deus vo, vel Deus aliqua petunt uru, ab bominibus receda , is, Duiufmodi funt, qua $f i-$ ni fomùi, $\mathrm{u}$, Deum offendo, of endere, of recedere . gnificant timere, off endere, ignificant auxilium prafiare, fuperioritatem, fubiectionem, Muita vero ver obedientiam, fuperioritatem, deruitutem, victcriam, of fimilia, regunt Da dindufaria. Jeruicacu ioni coietz, excedit alios fapientia o incufatiuum rei or $V$ erba verò dandi, of promittene vo curturu, dare pecunias

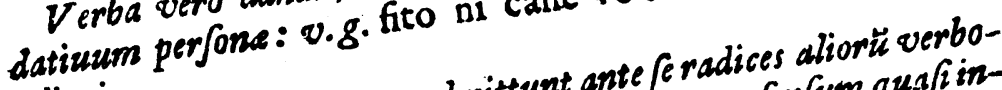
alicui. Multa /unt verb tunc predicta radices babent fenf is verb a ver:o rum immutatas; gici faji me, uru, audire incipio, is idem, tçuqe,

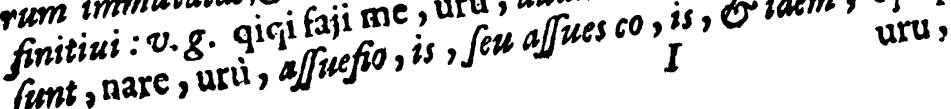


66

uru, fate, tçuru, finior,iris $D Y N T A X I$

incipio, is, tódoqe, uru, perfeuero , difco, is, sòme, uru, tçuru, dirigo, is, atiri, u, cafureperio, vel profequor, eris, ate $u$, pere, uru, dificile polfum, es, foconai, vage uru, diuido, e intenfe laboro goxi, $u$, excedo, is, fãgue , erro, as, fumaxi monai, nollo, is, as, àqi, u, fafidio, is, tai, voxi, u, multum uis finitis in $i$, facivero radices verborum antepolo, is, of tocile lectu of , faciunt quafi fupinum in anteponantur adieczitu, v. g. iòmi iafui, fadebent effe in genitiuo 1 fubfequatur illis nom idem petit particula, fö v. g. fito tçu no tō gen fubszantiuum fö do no fito, quot , fö do, quando fagnifícat , inum peccatum, v. ร. Nanban iòrinot funt bomines, idem petit , e, v. g. àru petunt etiam genitiuno mono, res Europe ; fed bit particula, iori titudinem vel paucum numeralia, feu nomina fic eft relatiuum. funt autem vel paucitatem : v. g. amàta no fina que dicunt mula, um, cazucina pradicza, bechi, alter, fito, multibomines, um, iro iro, mul multi, a, a, fama zam a , um, fon, proprius iffai, omnis, e, zdes, a, iorôzzu, quilibet , multi rnodus, a perfone nobiles, idem, vòno vòno, \& cotó $Q c$. izzure, quis, a, itçumo, fempex: igue, quod subfequitur, nocoto qu, reqi reqi, ne, ordinarius, v.g. itçumo no coto, id quod, quod remanet, to, vel fucoxi, a um, ima, mòdo, ifafaca, parum femper. tçucza petunt ante fo genitizun maturaliter, sòno fora, ext idem, só. uum, of tunc a genitiuum fa illis fub fequaiur nomen fub predifubstantiuum adiectiua debent reputarifí verio nom fub flantiv. g. iorözzu danco xite iecaro, bonum erit fum non petunt:
conflizum.

De Arithmetica Iaponix \& materia numerorum, in quibus hoc opus hic labor.

D po funt modi numerandi in lingua ifla I aponica prim per numeralia communia propria ipfus lingus prim cant, iomi, es bacperweniunt folum propria ipfius lingua, $q 7$ faciatis. 


\section{E A R I T H M E T I C A}

onum, of folent boc vti ad dicendum parum : v.g. sàqe fitòtçu nòmaxete tamóre, da mibibibere parum vini, futatçu, duo, mitçu, tris, iòtcu, quatuor, itçutçu, quinque, mutçu, fex, nanatçu, feptem, jàtçu, octo, coco notçu, nouem, to vo, decem, icutçu, Jgnificat, quot? in rebus que non babent propriam numerationem.

Secundus modus numerandi eft per vocabula, coie, ideft mëdieata a lingua Cbinenfi, boc veró non vtuntur vfque ad decem per $\int e ; n i f i$ rebus numeratis adiungantur, que quidem res debent IIgnificari vocabulis etiam Cbint/jibus; \& non I aponijs.termini nu merales funt, ichi, wnum, ni, duo, fan, tria, xi, quatuor, go, quinque, ròcu, Jex, xichi, Septem, fachi, octo, cu, nouem, jŭ, decem vtuntur per Se istis a numero ondecirno of Jupra, eft autem vndecim, juich, iiüni, duodecim, iŭfan, tredecim, júcul , decem \& nouem, is numeris denariys anteponzuntur numeri vqque ad decem:v.g. nijü, viginti, fànju, triginta, fanju ichi, triginta vnum, cuju, nonaginta, fiacu, vero Jignificat centum, fiacu ichi centum o vnum fiacu ju, centum \& decem, fiacu fanju, centum \& triginta, ni fiàcu, ducenta , sàmbiàcu, trecentum, xen, aul-. tem fignificat mille, xen ichi, mille \& vnum, xen roppiacu sànju ichi, mille fexcenti triginta onum.

Numeri vero Iaponiy antepofiti vocabulis Iaponix, que vocant iomi, et ablato, tçu, a numeralibus pradictis, fiue fint nomina fiue radices verborum quibus iunguntur numerăt res fgnificatas per talia vocabula : v. g. fito cotóba, vnum verbum, futa coto vari, duse rationes, midmi, tria retia vel tres retis miffiones, iocama, coquere quatuor vicibus in caldaria, itçucdiqe, quinque aggreffiones muicasàne, fex vefles feu coopertura, nànd catana foptem vulnerationes gladio.ià catägue, oczo onera, cùca vari noucm tranfmutationes, tò cula, decem differentic.poft numerum vero decimum, boc modo numerandi non vtuntur; fed dicunt, iro jŭichi, vel juichi no iro, ondecim colores . ad interrogandum vero eft verbum, icuitçu, fi verò res de qua interrogatur pofponatur, debet addi numeralibus particula, no, v. g. itçucu no qi zo quot ligna funt ? idem, qi icutçu 20 ? et respondetur, futatçu, dus, , mitçu . tria etc interrogatur etiam per , icutçu ablato, eçu pofita vero re numerata de qua interrogatur: v. g. icu tncoro, quot loca ? icu toqi , quot bore? fito fallaxi, unus ferme vel conwerfatio, futafugi dua linee, iote, quatuor marwusinter digla- 
diatores: v.g. itçutçu bu, quinque grana, mu tocoro, fex lo$c a$, iamavari, fex circuitiones, cunnai, nouem onera to modo guo I apones onus portant ante ơ reiro in ligno, tò vatari , decem irinfitiones: v.g. Or alia omnia qua numerari po.junt, idem autem eft dicere, mu tocoro quòd, mutçu notocoro o quod, tocoro mutçu, fex loca, fito ie, fignificat rem fimplicem, futa ie, duplicem feu duplicatan, miie, triplicatam orc. idem fit cum. numeralibus cbinen/ibus few, coye, adiunctis vocabulis etiam cbi$n:$ jous : $\dot{0}$ aliquando inisła numeratione nibil ammittnnt numepi aut res numerate; aliquoties autem vel alterum vol vtrumque aliquid committit vel mutat, o pracipue in numzeris primo, fecundo, tertia, fexto, decimo, í centefimo: ơ alia funt mutationes: bic autem porentur communiores; quando vcrò in particulari nibil fuerit adnotatum, eff fignum quod nulla est tranf. mutatio.

Interrogatio de bominibus fit $\vec{y} e r$, icutari? quot lsomines? Refponfio verì fit poltpanendo, nin, numeralibus cbirenfiùus: v.g. ichinin, vsus bomo, ninin, dwo, iortari, vero figrificat quastuor: quia, xinin, Jgnificat bominem rsortuum.

Interrogatio de diebus fit per, icca, quot dies? \& onus dicitur , fi fitoi, quia, ichinichi, Jignific at diem folarem integrum, futçuca, dus dies, micca, tres, iocca, quatuom, itçuca, quisque, muica, fex, ndnucà, feptem, iòca, octo, còconoca, nouem, toca, decem, fatçuca, viginti reliqui numeri dierum fun: pernumeralia, coie.

Numerus noctizm, eft pofponendo, ia numeratibus, coie, v.g. ichi ia, una nox, ni ia, duce frc. fit etiampofpofito, io, quod Jgnificat I aponice nodtem numeralibus, iomi . icuio. , quat zoczes? furaio, due, nanaio, feptem nockes $\mathcal{O} c$.

Numeratio men fum anni fit pos Zpofito, guat, numeralibus, coie, ,ed primaluna vocatur, xóguat, fecunda vero, niguat, textia, säguat, quarta, xiguat, ondecima veròdicitur, ximò eçurq, duodecima verò ơ vitima dicitur, xi vàfu, $\sqrt{i}$ autem velimuss numerare menfes abjolutè pofponimus, tçuqi, quod lunam. fignificat ntsmeralibus, iomi, ablato, tçu, interroganus vera, icutçuqi ? quot menfes 3 . \& refpondetur, fito tçuqi, onus., vfque ad decem, quod eft, totçuqi, poft decem veròfit numeratio per numeralia, coie, $v . g^{\circ}$ ju ichiguat, ondecimmen $\int e s$, adinterrogandum verò de menfe quisnam fit; I anuarius ne an Februa. 
rius? ft per, ndnguat, Primus I T E T I C A
luna Martÿ. Annurneratio annorum fit poflpoftom menis anni $I_{\text {aponen }} \sigma_{g}$ pondetur autem antepofito, nen, $v$ ito, nen, numeralibus, coie. tres, sò ionèn, tresinen, vnus, ionen, ndnnèn, quot anni? Refdraginta anni, fres vel quatuor, sòn, quatuor, fanganen, ni junen, vel, ni ju , Jzhnificat viginti anèn, triginta vel qua. icutoxi, vel toxi icutc toxi, vel tòxi ni jí, in in bominibus, ide nos bominum or anitçu, quot annos babet? interrogant autem, quorum bc. pofponendom perfectorum, bomerant etiam anunus, ni fai duo, fan zii, tai, numeralibus, boum foilicet, ofeAnnumeratio vicium fit pos anni. v. g. nindo, quot vices s ichidpo fito, do, numeralibus, coic,
quinquies, tai fan, bis velter.
Annumeratio nauium iôdo, quatuor, gôdo, v. g. nainzo, quo nauium fit po zô, tres fallồ, oczo, naues? Refpondetur : iffô, numeralibus, coie, Ichiren, rza linea, dú, decem. mav'garitarum, orc. Ananzomeratio or. reift po/pofito, fen, num, tractatuum, vel repetitionum eiufde
duce, fanben, tres as iema ria fidcu: 0.8 . ippen, ona, nifen.
ginta aue maria.
Annumeratio. ginta aue maria. libus zres. vinus aus momme, vnus, postponendo, itaque, ip autem argenteus dis, ni mòmme, momme, numeraexpradictis parn, frnificat decimation in decem que ran mòme, preaicilis dectibus, gofùn, me partem argentei, vocant ippun Decima gurtibus. cem quarum quoque verò pars argentei diuiditur adbucin alï in rocurin, xichirin, fachirin, ichirin, nirin, farin adbuc in alÿs de. elt argentei decima pars, fiacurin, \& flation est iòrin, gorin, decem mille, centum \& onus, funt centum argpun, quod tio, fut mille. funt etiam alice mos, icquan ne, mille vna ex illis mo fito, mai, vel moneta ex are, quarum icquanme

Ais monetis faciunt nondum, dua fan mai, tres cent. g. ichi mon,

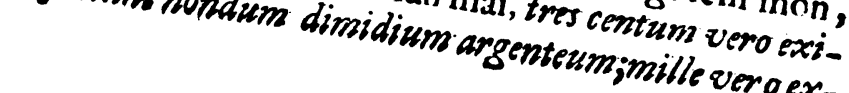


70 DE A R I T H M E T I C A
A dictis monetis vosant, icquan, jicquan, verò decem mille

or co.

Còreua ica födo ni or venditur 3 ni momme furu, decem vru, quanti pretij in momme ni iàfui, plus duabus dragmis osendi argentcos valer, poteft: vel duabio menjurarum tam reruciendam menfuram modij:

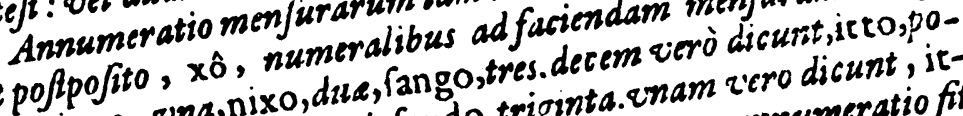
v. g. ixxo, ona, nixo, duse, fango, tres. decta.enam z'cro dicunt, itfpofito, to, nitò, viginti. Taldo, trartes, quilarum annumeratio fit to, quă diridunt adbuc in dec g. ichigo, vna, nigo, due, fango poftpofito go, nữzeralibu menfura ơ dimidia ex menfura, fatto,

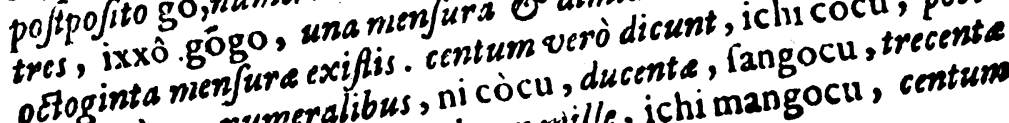
pofito còcu, numeralibus, ni còcu, dille, ichi mangocu, centum mille. Annumeratio menfura Statuum bò firo , futa firò, due, jippinumeralibus, ormi, v.g. Gò palmorum faciunt pofpofito, $x a c u$, numeralibus, coie, v.g. ixxacu, onus vero facit anum ftatum $H_{1}$ /pani vocant fanjacu, tres, fito firo, fex vero ex menfuris quc qui vocatur ot dictum tertia, faciunt menfuram, decem, fanguen, tres. vocatur, xach, una, nicqen, due, iccqen, decem, foeant, icchó, ex fex aginta uero ex iftis fit alia, jicchio decem, fangio, tres. ideft unus callis, niccho dufis fit una leuca feu miliare ex miliaex fexaginta uero tribi I aponie, quod uocant, ichiri, poitpofito, ribus partis fuperioris Iapiri, duo miliaria, lan, fignificat culum, ri , numeralibus, jecem, ,ori, quatuor: quia xir, iòco tan miqi tate ique, juri, decem, dimidiam leucam dicunt : $u$ transuerso uero dimidiam fan michi, dimidabet unam leucam; ex tran fo futa fro, babet duos

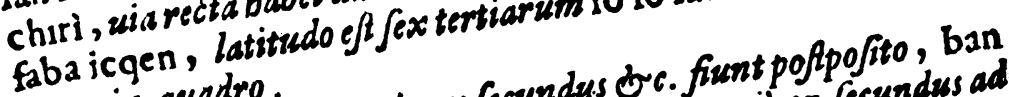
fla!us in quadro. numeralibus, coie, u.g. ichi ban, prime, pradictis: u.g. xinumeralibus, coie terminum additur, me,
fignificandum uero ter , quartus. fit etiam numerus cardinalis anteponendo, das 
nusmeris, coie : u. g. daiichi, primus, daini, fecundus, bc.

Annumeratio duplicium fit poßpofito, bai, numeralibus: $u$. g. ichi bai, duplum, nibai triplum, fanbài, quadruplum, fiàcu zóbai, centuplum.

Annumeratio partium ex tot una, fit anteponendo numeralia ad, buichi, u.g. ni buichi, ex duabus partibus una, fan buichi ex tribus zona.

Ad decimandum uerò poftponitur, vàrl, numeralibus: $u g$. ichi vdri ; ex decem partibus una, xi viri gobu, ex decem partibus quatuor $\delta$ dimidiam, ju buichi, autem coincidit cum, ichi vàri.

Numeratio remorum arcabufierum of corum que funt longa ut ligna fit poßppofito, chó, numeralibus: u.g. icctó unum, nichó, duo, fangio, tria, jichió, decem.

Annumeratia pifcism of lignorum ad comburendum \& $c$. fit postpofito con, numeralibus: $u . g$. iccon, unus fangon tres jiccon, decem, fiaccon, centum, fiacu go ju fangon, centum quinquaginta tres. tot prendidit Săctus Petrus or cum tanti efjent non eft ciflum rote.

Annumeratio foliorum papiri, uittarum argenti \&c. fit pof $l-$ pofito mai, numeralibus: u. g.ichi mai,una, cami gomai, quinque folia papiri.

Annumeratio tabulatorum que funt pauimenta domus fit pofpofito, cai, numeralibus: u.g. nicai, unus, fangai duo, xicai tres, gocai quatuor ficut babent domus Matriti.

Annumeratio uerò uaforum et calicum quibus bibunt fitpoßpofito, fai , numeralibus : u. g. Ippai, unus potus, uel unum bauAum, ni fai duo, fanbai tria, jippai, decem etc.

Annumeratio telarum ex fericis: $u$. g. et aliorum fimilium fit pofppofito, tan, numeralibus:u.g. ittin, unum, ni tan, duo, fandan tres, jittan deeem, xichitan bunè, uocant nauigium quod sclo petit feptem

Dicitur etian pofpofito, mai, numeralibus: u. g. gomai, quinque, gomai bunè, nauicula que pro uelo petit quinque.

Annumeratio arimalium quadrupedum fit poftpofito, fiqi, nusmeralibus.u. g. ippiqi, somum nifiqi, duo ranbiqi, tria roppiqi, fex jippigi , decem, fiappiqi , centum , xenbigi , mille. 


\section{2- DE ARITHMETICA}

Annumeratio imaginum, picturarum, of medicinaram fit po/tpofito, fucu, numeralibus: $v \cdot g$. iprùcu, vna, ni fucu, duce fönbucu, tres, roppucu, fex, jippucu, decem ; ita annumerätur etiam acus.

Annumeratio librarum fit poftpofito qin, numeralibus:v g.icqin, ona libra, niqin, due, fanguin, tres, ròcqin, fex, jicqin desem, fijcqin, centum xengu:n, mille.

Annumeratio miffarum, \& congregationum bominum fit foApofito, za, numeralibus:v.g. ichiza, ona niza, dua, fanza, tres; juza, o melius, tòza, decem.

Annumeratio faccorum oriza, aut tritici etc. fitpofipofito, fiô, numeralibus : v. g. ippiô, vnus, ni fiô, duo fanbiô, tres, xi fio, quatuor, roppio, fex , jippio, decem, fiappin, centum, xenbiô, mille.

Annumeratio lignorum, arundinum, acuum, fit poftpofito fon, numeralibus: v.g. ippon, vnum, ni fon, duo, fanbon, tria, roppon, fex, jippon, decem, fiàppon, centum, xenbòn, mille.

Annumeratio fa fciculorum fit poftpofito va,numeralibus: v.g. ichi va,onus; ni ua, duo, fanba, tres, jippa; decem jüichi va; vndecim ; ni jippa; uiginti.

Annumeratio onerum feu farcinarum; quas equi portant; fit pofpofito; só; numeralibus : u.g. iffó unum; nifo duo ; fanzó trix; ;iffo; decem. eodem modo numerantur illa inftrumenta ques uocantur; biobu; duo enim Seu par ex illis uacatur; ifió ; etc.

Annumeratio uero eius quod unlgo dicimus mano de papel fit postpofito, g1ô ; numeralibus: u.g. ichi gio; una nigio ; dưa, fangiô tres; ufque ad decem qua fit poftpofito ; locu, numeralibus: u.g. iffocu decem, feu ona qua uulgo uocatur media refma, ni facu; viginti que erit refma integra cum ifla particula; focu posipofita numeralibus annumerant etiam par calceorum: $u . g$. iffô cu, par calceorum .

Annumeratio fubflantiarum fit pofppofito, tai, nutrieralibus: u. g. ittai, una ; nitai duse; fandai ; tres; Deus no von tocoro va goittai de gozaru; Deus in quantum Deus eft ona fubftantia et elfentia $c$

Annumeratio capitulorum fit pofpofita cagiô , numeralibus:

u.g. 
v.g. iccagiô rinum, nicagio duo, fangagio tria, roccagio fex, fiaccagio, centum.

fixin wheratio guttarum fit poftpofito, ti qi, numeralibus: $v$. g. itriqi , wna gutta, jisteqi, decem.idem jit poftpofito, xiz<ucu numoralibus, iomi ; v.g. fito xizzuca, vna \& c debet auferri, tçu, a numero vt videtur.

Annumeratio paxillorum quibus comedunt, \& eorum qux bina \& bina portantur, fit postpofito, tçui, numeralibus: v.g. itçui, vnum par, jittçui, decem .

Annumeratio farcinasum bominam fit postpofita, ca, numeralibus: v.g. icca vna, nica dua, sànga, tres.

Annumeratio librorum fit poftpof to, quan, numeralibas: $v$. g. icquan vmus, niquan duo, sanguàn tres, ròquan $\int e x$, jiquan decern.

Ad interrogandurn verò anteponitur, nan, nominibus mutatis vel non literis ficut in numero tertio: $v . g$. àno mmadòmo va nanbigi zo ? quot funt illi equi ?

Annumseratio rcgnorum fit poftpofito, cacocu, numeralibus: v. g. icca cocu, vmum, ni cacocu diuo, sangacocu tria, jiccacocu, decern.regna vay diziduntur in prouincias feu diftrictus quos vocant postpofito, gun, numeralibus: v.g. ichigun, ona prouincia, migun, due, fangun, tres ofc.

Sermones vero \& exbortationes annumerantur postpofito, dan numeralibus: v. g. ichi dan, vnus fermo vel concio, verba vero annumeranzur poftpofito, gon, vel guen, numeralibus: $v g$. ichigon vmum, fanguen, wow nowhe.

Pofspofita particula, zzutça, numeralıbus ıuss , coie, quam iomi, fit fenfus binzus, $a, u m, v . g$. ichinin ni vxi lanbiyi izutç vo toraxeta, vni dedit tres vaccas, ichinin zzutçu faque sàubäi zzutçu vo nomarèta, vnufquifque bibit tra baufla vini.

Ad loausendum diuifase duo vel tria: v. g. copulant duo numeralia : v.g. xígonin, quatuor vel quinque bomines, catera ex bis elicies.

Particula bonnis funt quatuor, vo, von, go, mi, duse prima iunguntur vocabulis, iomi, vitima verò iunguntur vocabulis, coie, fuze chinenfibus. vltima eft bonoration \& illa vtimur ad loquendum de rebus diuinis: v. g. midexi tachi, difcipuli Chrifti Domini, goichi nin vocoite cudalarei, mittatis obfecro vnum ex dominis. 
74

D E A R I T H M E T I C A

$V$ erba verò qua fequntur etiam fi babeant particulas bonoris ; babent fe tamen ex parte loquentis; bonorem verò important inquantum perfonan cum qua loquimur vel de qua loquimur attinsunt: v.g. go focô, quod/g̈nificat feruitium, von furu mài, quod eft conuiuium, von cotóba, quod gf verbum feu fermo, von monó gatàri, quod eft conuer $\int a t i o$, von natçucaxij, vel von nocori vo voi, babere quod Lufutani vocant faudades vel Hifpani cariño, von tòri auaxe, quod eft interceffio, ron mi mai, quod eft eifitatio, von cha, quod eft quidam potus quo inuitant, go dancó, quod efl confultatio feu congregatio ad confilium capiendum, von rei, quod eft gratiarum actio, von busata, quod est defectus in urbanitate, vomòtenaxi, quod ef bene b laute tra. ctare, go chifo, quod eft aftimatio, go iqen, quod eff confiliurs v.g. fabacari nagara go iqen vo moxitai, \& fifit inuerecundum o indecens volo tamen confilium tibi dare ors.

\section{Aliqux regulx coniugationum in friptura librorum.}

\footnotetext{
A Liquando fit verbum affirmatisum cum prefenti negatius A fupra pofito; ablato u, in quo finitur:v.g.oracio vo tçutomen tòqi va, quädo babeo orationem, xòfa vo tçutomèn tàme ni va, ad exequerdum opus, michi bigi tamavanto voboxime$x i$, ad illum deducendum boc

Ad futurum attrmatiuum additur particula, bèqi, affirmatiuo fuprapofito ablato, $\mathrm{ru}, \boldsymbol{\sigma}$ ad futurum negatiuum additur, bicarazu, affirmatiuo: v. g. mòfu beqi, dices, mólu beqarazu, non dices. $/ 2$ vero oratio finitur in futuro, bèqi, conuertitur in bexi.

Infinitiuum futuri fit addito, còto , futuro : v. g. iòmu bèqi coto, fubiunckiuum fit pofpofito, qerëba, radicibus verborum v. g. fugure qeréba.

Gerundium in do fit pofpofito, te, radicibus:v.g. qiqi tamaite.

$\checkmark$ erbum fubfantiuum in friptura librorum ef, ndri u or qeri $u$, f fin illc finitur oratio eft in radice: $v . g$. fadime naqi io no ixei nari , ef dignitas mundi qui non babet flabilitatem .

Irateritum eft pofpofito, ari, $u$, radicibus: $0 . g$. süguretaru,
} 
D E A R I T H M E T I C A

earu, $f$ verò oratio in illo finitur remanet, ari, $u$, in radice : v.8. súguretari.

Prateritum plufquam perfectum ef pofpofito nari, trafinti: v. g. ägue tamo nari, adorauerant.

Si forte fuerint aliqua alia regula in librorum foriptura. erunt ita faciles quod facillimo negatio illas confequentur qui in lingua Iaponica fuerint tam prouecti, ot iam eius librorum lectioni foffint vacare.

\section{ERRATA SIC CORRIGE.}

\section{Primus numerus paginam, fecundus lineam indicat.}

pag. 3. linea 2. iu. lege in. 7.25. niffion. l. niffon . 10. 7. goraru . l. gozaru . 14. \& 15. accentus fupra, 0 , funt acuti o debent effegraucs. 16. 5. mainne1s . 1. mainen . 19. 24. loca . 1. 60co .24. anstepenultima. de gozate. l. de gozatte. 28. 12 . égue l. ägue . 33.22. fucacaranda . l. fucacarananda. 3 3.28.cuuaav l. cuvazu. 34. antepenultima . incadeca. l. icädeca. 36. pemultima . voi cotaranu . l. vo cotaranu. 39. 25 . cu varuu. l.cui maraxenu . 40. anteperultima amittit . l. admittit . 43. 10. antepensultima particula. na. 1. polipofita particula. va. 42. 32. uobrou. l. noboru. 42.11. vgetm vari . l. vge tamavari. 48. vitima. voforozij . l. voloroxij. 49. 1. voforoxi. voloroxij. 49. 26. Jignificat. 1. Jignificant. 50. 10. doco . 1. coco. 51. 3. Poca. 7. foca. 57. 26. teugu. l. tengu. 60. 5. tocude. 6. to coröde . 60.19. vo xite. l. coxite. 60.24. mate. ommittatur et non legatur. 61. 34. ägne. l. ägue . 64.15. ni va genbur. I. ni va vo qenbut. 64.19. geuzan. 1 . guenzan .67.14. iuni, iulan, d.juni,jufan. 70.31.culun. l.anum. 


\section{A \\ Grammar \\ of the Japanese Language}

FOR THE SAKE AND HELP

of those who wish to go to the Kingdom of

Japan to preach the Gospel.

Composed and dedicated to the Blessed Congregation for the

Propagation of the Faith by Brother Didico Collado,

O.P., who was for many years in that Kingdom

as a Minister for the Propagation of

the Catholic Faith.

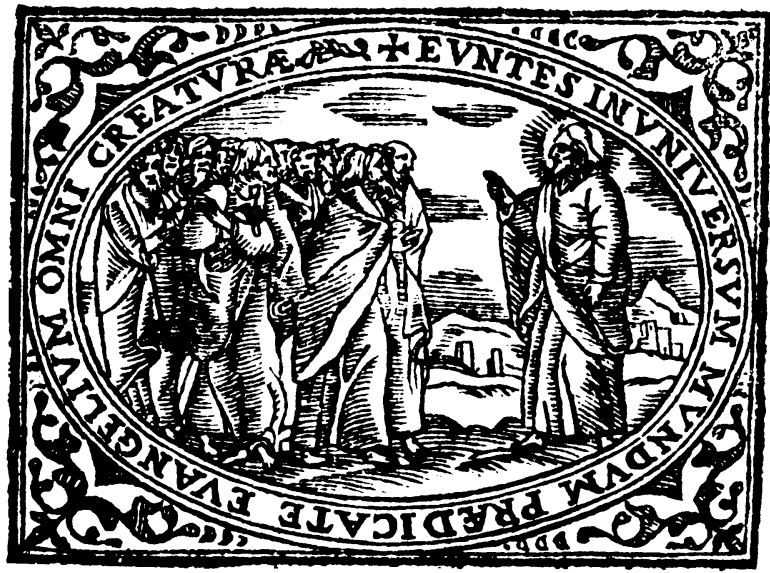

Printed by the Blessed Congregation for the Propagation of the Faith. 1632

BY PERMISSION OF THE SUPERIORS. 
It may be published if it please the Most Reverend Father, Master of the Holy Apostolic Palace.

For the Archbishop of Umbria. The Vicar General. It may be published.

Brother Nicolaus Riccardius, Master of the Holy Apostolic Palace, Order of Preachers. 


\section{Prologue to the Reader}

\section{With Some Advice on the Correct Pronunciation of the Japanese Language}

Long ago, at the beginning of the establishment of our orthodox faith in the Japanese kingdom, a grammar of this language was made by Father Ioannus Rodriguez of the Society of Jesus. ${ }^{1}$ However, since things rarely turn out perfect at first attempt, and, because of the passing years that have made it difficult to find a copy of this grammar; I thought that it would honor God and the ministers who preach the faith (which cannot be taught without the use of language) if I were to select examples (and there are many) that are useful to this language, neglect those not accepted by the experts of the language, add, with the help of God (who gives words to the evangelists), the words that I have learned from experience, practice, and continuous reading, and by such means offer up a handbook of the Japanese language in which I would bring together in a brief span these examples with those precepts which the preachers (for whom I began this work) need to learn of the Japanese language. This is done because examples are necessary with the rules and precepts so that it is possible to demonstrate the rule which has just been explained. Not only have these examples been selected for the greater help and enjoyment of the students, but also their explanation has been added in Latin (which is the language most common to theologians); thus the teacher will have very little left to be desired. Even if all the elements included in this grammar, as in the dictionary (which with the help of God I plan to publish shortly), ${ }^{2}$ are polished enough and sure enough to be used with trust; I would still want them to be submitted to the judgement of the reader so that the preaching of the faith, carried on with a more correct language, may become more fruitful.

When two vowels follow each other in any Japanese word, they are not pronounced as in the Latin word valeo or in the Spanish, vaca, but each is pronounced independently; $v, a ; v, o ; v, i$.

${ }^{1}$ This Reference is to Arte of 1604-8. The Arte Brev'e, printed in 1620 in Macao, was not available to Collado.

${ }^{2}$ The Dictionarium sive Thesauri Linguae Iaponicae, which was in fact published at the same time. 
The letter $s$ is pronounced as ş̧; e.g., susumuru, sçuş̧umuru.

The letter $j$ is pronounced smoothly (blande), as in the Portuguese joaõ and judeo.

The letter $x$ is also pronounced smoothly, as in the Portuguese queixumes.

When there is the $\operatorname{sign}^{\wedge}$ over the letter $o$ it is pronounced ou with the lips almost closed and the mouth partly closed; e.g., buppô.

When there is the sign ${ }^{\vee}$ or ' over the letter $o$ it is pronounced with the mouth open as if there were two letters, oo; e.g., tenxó or gacuxö. ${ }^{3}$

If the signs we have just shown are over the letter $u$, it is pronounced long as if there were two letters, uu; e.g., taifú or aiaú.

When the sign $\sim$ is over the vowel, the sign should be pronounced like an $n$, not strongly but swiftly (cursim) and softly (leniter); e.g., vãga. ${ }^{4}$

$Q e$ and $Q i$ are written without $u$, because when $u$ follows $q$ or $c$ both letters are pronounced as a sinalepha; ${ }^{5}$ e.g., quódai or quainin.

When $u$ follows $g$ and immediately after the $u$ is the letter $e$ or $i$, it should be pronounced as in the Spanish word guenin; but if the letter $e$ or $i$ follows $g$ immediately without the $u$, it should be pronounced as in the Italian word giorno; e.g., xitãgi.

The letter $z$ is pronounced with the same strength as in the Spanish word zumbar; e.g., mizu.

But if there are two $z z$ then they are pronounced more strongly; e.g., mizzu.

When there are two $t t, x x, z z, q q, c q, i j$, or $p p^{6}$ it is important to persist in order to obtain perfect pronunciation and the exact value of the word; for mizu means 'honey' and mizzu means 'water.' Therefore, if the words are said with the same strength or the same gentleness they can mean either 'water' or 'honey.'

When ch comes before a vowel it is pronounced as in the Spanish chimera; e.g., fochó.

\footnotetext{
${ }^{3}$ See the Introduction for the regularized usage of these symbols in the translation. (The transcription of gacuxŏ, and the aiai below, are at variance with the rule for the translation and are here transcribed as printed.)

${ }^{4}$ This convention is not transcribed in the translation (cf. Introduction).

${ }^{5}$ More regularly synaloephy - the contraction of two syllables into one.

${ }^{\circledR}$ The geminates that actually appear in the text are; $t, x x, z z, c q, i j \& p p$, as well as $c c$ $(c c h), m m, n n$, and $s s$. Two appear initially $m m$, as in $m m a$ 'horse,' and $z z$, as in $z z u r u$ 'to leave.' The form $q q$ which would be phonetically equivalent to $c q$ is not recorded.
} 
But if $n h$ comes before a vowel it is pronounced as in the Spanish maña; e.g., nhuva.

The letter $f$ is pronounced in various regions of Japan as it is in Latin. In others it is pronounced as if it were an imperfect $h$. For both pronunciations the lips and the mouth should be nearly, but not completely, closed.

When $t c ̧$ is in a word (and it appears quite frequently) the student should pray that God have mercy on his pronunciation because the word is very difficult, and its pronunciation is not to be found ( 5 in any other language. It is not truly pronounced $t_{\zeta}$, nor as $s$, nor as $c$ alone, but rather by striking the tongue violently against the teeth in order to pronounce both $t$ and $\varsigma$, but with more $\xi$ than $t$ seeming to be sounded; e.g., tçutçumu.

The letter $r$ is said smoothly and softly everywhere it is found, either at the beginning or in the middle of a word; e.g., ranguio, or cutabiruru.

$Y a, y e, y o$, and $y u$ are pronounced as in Spanish. ${ }^{7}$

When words ending in $i$ or $u$ are pronounced by the Japanese, the last letter is almost not heard by the student. For instance, if he hears gozaru he will think he hears gozar, if he hears fitotçu he will believe he hears only fitotç, and when he hears axi no fara he will perceive only ax no fara.

When a word ending in a vowel is followed immediately by a consonant, particularly $b$ or $s,{ }^{8}$ between that vowel and consonant is pronounced the letter $n$, not perfectly, but softly; e.g., sonõ gotoqu.

I have given special care to the accenting of words. ${ }^{9}$ This has been done so that the signs that have been placed correctly over the accented letter will allow the listener to understand the meaning of the words and the sentences of the speaker. For instance, qèixèi has the accent on both $\grave{e}$; fibicàxi has it on the first $i$ and on the $a .{ }^{10}$ This same arrange-

\footnotetext{
${ }^{7}$ This sequence is not used in the body of the grammar, rather the less phonetically accurate $i a$, ie, etc. It should be noted that the Dictionarium, which was written contemporaniously, does use $y$ for the semivowel.

${ }^{8}$ For $s$ read $g$. The Arte $(177 \mathrm{v})$ discusses this phenomenon as being characteristic of vowels before $d, d z$, and $g$.

${ }^{9}$ Since in fact the accent has been carelessly recorded in the text-in places added in an almost random fashion by either the author, his helpers, or the printer-we have not included its marking in the translation. (Cf. Introduction.)

${ }_{10}$ The Dictionarium has the spelling fibicaxi in one entry and in the only other it is transcribed as above.
} 
ment will be respected in the dictionary, with the accent being written with the same degree of correctness as is able to be achieved with great attention. If at times I have made mistakes, I am prepared to correct them immediately. Concerning what has been explained too briefly or left out of this grammar and the dictionary, learned people will be able to do that when they add a third dictionary and a third grammar, since it is easy to supply this material. Because I wish neither to be criticized by the Head of our Order (pater familias) and the Lord our God, nor do I wish this knowledge to be wrapped up in a handkerchief; ${ }^{11}$ I want by these two works to help and to cooperate in the salvation of the Japanese not only by preaching but also by offering to the preachers, if I can, the tools of the language and chiefly the method by which they might better learn the Japanese language, a task made very difficult by the persecutions in Japan. Farewell, Reader, and be of good cheer. Madrid, 30 August 1631.

\footnotetext{
${ }^{11}$ Acts, 19:20. Referring to the servant in the parable of the pounds who is condemned for kecping his money "laid away in a napkin."
} 
In This Grammar We Have for the Most Part Observed the Arrangement Which Antonius Nebrissensis and Others Have Followed in Latin for the Treatment of Sentences, Namely, Nouns, Pronouns, Etc.

\section{The Noun-Its Declension and Its Gender}

In the Japanese language there are no case declensions as there are in Latin; but there are certain particles, which when suffixed to nouns, determine the differences between the cases for both common and proper nouns. The particles which form the nominative are five; $v a$, ga, cara, no, and iori. The particle $v a$ is used when we want to give a sort of reduplicative ${ }^{12}$ and specific turn to the person or thing that is signified by such a noun. It indicates either the first, second, or third person; e.g., Vatacuxi va mairanu 'I, or those related to me, will not come.' The particle no is suffixed to the second and third person, especially if they are inferior in rank or in a sentence where there is a relative construction which does not indicate a transitive action; e.g., sonata no móxita coto 'that which you said.' The particle no is also used when some indefinite form is used; e.g., iie no aru ca miió [. . . miio] 'see if there are houses.' The particle ga is used usually for the first and third persons of inferior status as well as for the second person when he is the most lowly or is to be humiliated; e.g., Pedro ga qita 'Peter came.' This particle is also used to indicate something indefinite, as has been said of the particle no; e.g., coco ni va iie ga nai ca? 'aren't the houses here?' It is also used in sentences that have a relative construction which does not indicate a transitive action. If the reference is to something of inferior or humble status the particle $g a$ is also used; e.g., soregaxi ga caita fumi 'the letter which I wrote,' sochi ga iúta coto 'what you said.' The particles cara and iori are used to form the nom-

\footnotetext{
${ }^{12}$ The text uses reduplicatiuns, with the grammatical meaning of plural singular; e.g., the singular I with the meaning of myself and those around me.
} 
inative case when the sentence shows a transitive action, especially if the sentence contains a relative construction; e.g., Deus iori cudasareta gracia 'the mercy which God gave,' tono cara core vo vôxe tçuqerareta 'the Lord taught this.' Sometimes the words are in the nominative case without any particle; e.g., Pedro Ioaõ vo iobareta 'Peter called John.' There are two particles for the genitive; i.e., no and ga. The particle no is used for all persons of superior rank; e.g., Padre no vó qiru mono 'the priest's clothes, or habit.' The particle ga is used for people of inferior rank; e.g., Pedro ga fumi 'Peter's letter,' sochi ga mono 'your thing,' are ga cane 'your money,' tono va iocu ga fucai fito gia 'the Lord is of great cupidity, that is to say he is very eager.' Sometimes the particle to is suffixed to the genitive; e.g., Pedro no to degozaru 'it belongs to Peter.' But since this is not a perfect way of speaking, it is better not to use it. I have cited it so that if you hear it you will understand. When two nouns are joined to form a single word, the one which is like an adjective does not require the genitive particle; e.g., cocuxu 'the Lord of the kingdom.' According to the ordinary rule we should say cocu no $x u$. This way of forming the genitive is very common in Japanese; e.g., Maria coto 'Mary's thing.'

Two particles form the dative; i.e., $n i$ and ie. For example, Pedro ni móxita 'I told Peter,' Padre ie ague maraxita 'I gave, or offered, it to the priest.'

There are five particles which form the accusative case; i.e., vo, $v o b a, v a$, ie, and $g a$. The first, vo, is the most used; e.g., Pedro vo iobe 'call Peter.' $V a$ is used when one wishes to express in particular a noun in the accusative; e.g., niffon guchi va xiranu ${ }^{13}$ 'I don't know Japanese.' The ending $v o b a$ is the same as $v o v a$, changing the second $v$ to $b$ they use it as va; e.g., fune voba nori sutete; cane bacari tori maraxita 'abandoning ship, I took only money, or gold, with me.' $I e$ is used to indicate the place to which one goes; e.g., Roma ie mairó 'I go to Rome.' $G a$ is used for nouns which indicate non-living or humble things; e.g., are ie gozare, mono ga móxitai 'go there! I have something to tell you.' The accusative is also formed without any particle, as has

${ }^{13}$ Both the Dictionarium and the Vocabulario have either Nifon or Nippon, but do not record this form. It seems not to be a simple typographical error since the spelling is used in the title of the companion piece to this work, the Confesion, and since the text itself has niffion and it is changed to niffon in the errata. Nifon appears on page 43 . 
been shown in the example second before last, where the second accusative is without a particle.

The vocative is formed with the particle icani. It is not suffixed to words as are the other particles but it is prefixed instead; e.g., icani qimi core vo goronjerarei 'look at this, My Lord.' ${ }^{14}$ Usually, however, the vocative is formed without any particle; e.g., Padre sama (8 qicaxerareio 'listen, Reverend Father.'

There are three particles for the ablative; i.e., iori, cara, and ni. The third indicates the place in which; e.g., iglesia ni gozaru 'he is in church.' Sometimes ni is used after no; e.g., sonata no ni xi aru ca? 'are you going to make it yours, or take it for yours?' But this seems much more a dative than an ablative. The particles cara and iori are more common in the formation of the ablative; e.g., Madrid cara maitta 'I came from Madrid,' Pedro iori corosareta 'he was killed by Peter.'

There are four particles used to form the plural. They are placed immediately after the noun they pluralize and before the particles which indicate case. These four particles are tachi, $x u$, domo, and ra. The first, tachi, forms the plural of those noble things which one wishes to honor; e.g., tono tachi 'lords.' The particle $x \boldsymbol{u}$ forms the plural for noble things but not those of the highest rank; e.g., samurai $x u$ 'nobles (nobiles), but not lords (domini).' The particle domo is suffixed to words which indicate humble things, either abstract, animate, or inanimate; e.g., fiacuxó domo 'farmer,' ixi domo 'stones,' $m m a$ domo 'horses.' The particle $r a$ forms the plural of nouns which indicate very low things which are to be despised; e.g., Iudeo ra 'Jews. ${ }^{15}$ The case particles which are required by the sentence are placed after the pluralizing particles; e.g., tono tachi no coto domo vo varú iú na 'don't speak badly about the Lords' affairs.'

There are some words that are plural in themselves; e.g., tomo gara means 'men,' Nan ban mono 'European things,' Nan ban mono vo fomuru na 'don't praise European things.'

The particle icani, which as has been indicated above forms the vocative, is not placed after but always before the pronouns which are

\footnotetext{
${ }^{14}$ The Arte and the Vocabulario use the forms goran and goro in free variation. Collado here and in the Dictionarium uses what appears to be the less phonetically accurate transcription. The Spanish manuscript has goranjerarei.

${ }^{15}$ May I submit this as a candidate for the most exotic bit of anti-semitism in Christendom.
} 
made plural, while the particles which form the plural are placed after; e.g., icani Padre tachi vo qiqi nasare io 'listen to the priests.'

But two of the four particles which form the plural, domo and $r a$, are with certain words singular. Varera and midomo mean 'I.' Sometimes both are found together in the singular; e.g., midomora 'I,' midomora ga 'my, or mine.' The particles domo and $r a$ are also suffixed to the singular when one wishes to humiliate the thing mentioned; e.g., hara domo ga itai 'I have a stomach ache,' asu domo va aru mai 'tomorrow will not come,' asu ra va naró mode 'tomorrow will perhaps not come.'

The particle $v a$ is suffixed to singular and plural nouns which already have a particle; e.g., coco ie va mairanu 'he will not come here,' coco cara va denu 'he did not go out from here,' coco ni va aru mai 'he will not enter here.' Sometimes $v a$ replaces the particles of the declension; e.g., fune de saie ióió tçuita ni, cachi va nacanaca naru mai $(119 \mathrm{v})$ 'I arrived with such difficulty by ship: I would undoubtedly never have arrived had I come by foot, or on foot. ${ }^{16}$ The particle $v a$ here replaces cara.

Japanese does not have the genders feminine, masculine, and neuter as Latin does. There are, however, certain nouns which are feminine or masculine because of their meaning. Other nouns are common to both these genders. For things which do not have a proper gender vo is placed before masculine nouns and me before feminine; e.g., voivo means 'male fish' and meivo 'female fish,' vojica means 'roe-buck,' melica [mejica] 'roe-doe,', coma means 'horse,' zoiacu 'mare,' xô means 'male hawk,' dai 'female hawk,' cotoi means 'bull,' meuxi 'cow,' votoco means 'man,' vonago, nhóbó, or vonna 'woman.' All these words are placed in the dictionary as they come to mind.

The nominal adjectives have no gender or declension but make use of the same particles as the nouns. There are however many and diverse adjectives. Certain ones end in ai others in oi, ei, $u i$ and $i j$. There are other, more proper adjectives, which are formed by adding no to nouns. When the first five types of adjectives are placed before nouns they are then properly adjectives and do not in any way alter the composition of

\footnotetext{
${ }^{10}$ The text reads funè-de, and apparently Collado is attempting to indicate both accent and nasalization at the same time. He does not continue this practice.

${ }_{17}$ The text has caper silvester 'the wild he-goat' presumably the capreolus capreolus which is similar in appearance to the Japanese deer, cervus sika.
} 
the sentence. But when they are placed after nouns they become more like verbs and are in fact conjugated like them; e.g., tacai iama 'a high mountain,' xiguei ideiri 'frequent comings and goings,' caxicoi fito 'a wise man,' cavaij mono 'a wretched thing,' aiaui coto 'a dangerous thing,' umare tçuqi no cuchi 'one's natural, or mother tongue.' There are also adjectives ending in $n a$ which, when they are placed before nouns, do not alter the construction; e.g., qirei na coto 'a clean thing.' All the adjectives, except those ending in no, change their form in some way when they occur before verbs. Those that end in $a i$ change to ó; e.g., cono iama va tacó gozaru 'this mountain is lofty.' Those ending in ei change to ế; e.g., cono iama va xigueô gozaru 'these mountains are dense.' Those ending in oi change to $\hat{o}$; e.g., caxicô gozaru 'he is wise.' Those ending in $u i$ change to $u$; e.g., xei no ficú gozaru 'he is small in stature.' Those ending in $\ddot{i i}$ [ij] change to $i u_{\text {; }}$ e.g., caiu gozaru 'it itches. ${ }^{18}$ Among those adjectives ending in $i j$ there are many which come from verbs; e.g., nozomi,u means 'to wish', and from it comes nozomaxij 'which is to be wished for.' Other adjectives come from nouns; e.g., varambe means 'a child, or infant,' and from this comes varamberaxij which means 'childish.' Other examples may be found in the dictionary.

Adjectives which end in $n a$ change the $n a$ to $n i$ when they are placed before verbs; e.g., fuxin ni zonzuru 'I think it doubtful.' The adjectives that end in no sometimes change the no to na; e.g., bechi no fito changes to bechi na fito 'a different man.' Sometimes when it is followed by a verb the $n a$ changes to a $n i$; e.g., bechi ni gozaru 'it is different.' However, the meaning remains the same whether the word ends in na or no; e.g., bechi no fito no cuhi cara quita [...cuchi ...] is the same as bechi na fito no cuchi cara qiita'I heard it from the mouth of a different person. ${ }^{, 19}$ The only difference in these forms is that when the word ends in no no change occurs as a consequence of what follows. But, as has been said, those adjectives that end in $n a$ change to $n i$ when they come before a verb. If a substantive verb follows an adjective, it is an elegant statement; e.g., cono iami va tacó gozaru 'this mountain is high.' But if this kind of verb does not follow, the sense

\footnotetext{
${ }^{18}$ While this rule is operative for caij, it creates difficultics after $x$. Rodriguez' rule is ij

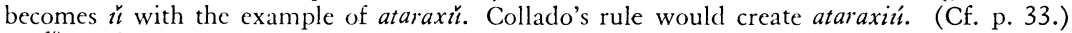

${ }^{19}$ Neither Collado nor Rodriguez make a clear distinction between the quantitative function of no and the qualitative function of na.
} 
is not altered since the adjective is used as a substantive verb. But this is not used before superiors. To them we will not say cono iama va tacai but rather cono iama va tacó gozaru. The same is true for the other adjectives.

Adjectives usually end in $i$ but infrequently these adjectives change to $x i$ or to $q u$. Ioi, which means 'good,' changes to ioqu, or ioxi; e.g., ioqu dancó xite, which has the meaning of 'offering good (11 council.' ${ }^{20}$ There are ennumerable nouns which become adjectives if $n a$ is suffixed to them; e.g., afo means 'ignorance' and from it comes the word afo na which means 'ignorant,' jiiu means 'liberty' and jiiu na means 'which is free.' Other examples are offered by the dictionary.

There are certain abstract nouns which become adjectives when they precede a vocable (vocabulis) with the meaning of 'man'; e.g., jifi means 'pity,' but when the word jin is placed after it, it becomes jifijin 'a pitiable person.' Fin means 'poverty,' but when the word nin is suffixed to it, it becomes finnin 'a poor person.' In the same way, when one suffixes $j a$ to $f i n$, it makes finja, which also means 'a poor person.' The word ban means 'watch,' but if the word $j a$ is added to it, it becomes banja 'a careful person.' Many other examples can be found in the dictionary.

There are in Japanese certain words which are borrowed from Chinese, called cobita ${ }^{21}$ or coie, and are written together to form by their union a noun and an adjective. Thus, ten mean 'heaven,' $x u$ means 'lord,' and tenxu means 'lord of heaven.'

The preterit of verbs (which will be taken up in their place) seem to have the same strength and meaning as adjectives when they are used before nouns; e.g., iogoreta te 'dirty hands,' where iogoreta is the preterit of the verb iogore,uru 'I became dirty.' Caita qió means 'a written book' and caita is the preterit of the verb caqi, $u$ 'I write.' The abstract (abstracta), or root from which the verb is formed, is itself a noun which signifies the action of the verb in the abstract; e.g.,

\footnotetext{
${ }^{20}$ Collado usually make a clear distinction between colloquial and literary forms. $\mathrm{He}$ apparently is suggesting that these non-colloquial forms are heard in the spoken language. Here, not only is the style left unexplained, but the translation faciendo bonam consultationem is less than ellucidating. Here the ioqu is in fact adverbial.

${ }^{21}$ From kobu 'to flatter.' An abbreviation of kobita kotoba, and used to indicate refined speech; i.e., that speech containing Chinese borrowings. See Doi Tadao, Kirishitan gogaku no kenkyzi (Tokyo, 1942, pp. 67-70). The term is also found in the introduction to the Vorabulario in the expression palauras Cobitas.
} 
facari means 'measure,' and it comes from the verb facari, $u$ 'I measure' while fajime means 'beginning,' and comes from the verb fajime,uru 'I begin.' Others will be found in the dictionary. The prepositional particle mono, when placed before an abstract or verbal noun, forms a noun which indicates the subject who does the action; e.g., mono before caqi makes monocaqi 'one who writes.' This same particle when placed after a root forms a noun which indicates the effect of an action; e.g., caqimono 'a writing.'

The particle goto placed after these same roots forms a noun which means a thing which is worthy of the action indicated by the verb; e.g., $m i$ is the root of the verb mi,uru 'I see,' and migoto is 'a visable thing, or a thing worthy of being seen'; while qiqi is the root of the verb qiqi,u 'I hear,' and qiqigoto means 'a thing which can be heard, or is worthy of being heard.'

If we place certain substantive nouns after certain of the verbal nouns about which we have been speaking, there is formed a noun which has the meaning of the action; e.g., foxi is the root of the verb foxi, $u$ 'to dry under the sun'; but, if ivo 'fish' is placed after it, the meaning of the expression foxiivo becomes 'fish dried in the sun.'

When the particle dógu 'instrument' is placed after the root of a verb it forms a noun meaning the cause or instrument of the action indicated by the verb; e.g., varaidog $u$ 'the cause, or instrument of ridicule,' caqidógu 'a writing instrument, or an instrument for writing.'

The particle me when suffixed to a verb forms a noun which indicates the terminus of the action; e.g., avaxe is the root of the verb avaxe,uru 'to unite or join two things,' and avaxeme means 'junction.' The same is true of other forms.

An abstract noun can be formed from those adjectives ending in $i$ if the $i$ is changed to sa; e.g., nagai means 'is long,' and nagasa means 'length.' The adjectives ending in $n a$ change the $n a$ to $s a$ in order to form abstract nouns; e.g., aqiraca na which means 'clear' will become aqiracasa 'clarity.'

Sometimes from two nouns taken together, often with a change in the first or last letter, there is formed a third noun, which is quasi-descriptive (quasi connotativus), almost like an adjective or noun with a 
genitive; e.g., from $q i$ 'wood' and fotoqe 'idol' there results qibotoqe 'wooden idol,' with the $f$ changed to $p[b]$. But if the prefixed noun ends in $e$, this $e$ is changed to $a$ in the attributive of the compound; e.g., tçumasaqi 'the tip of the nail,' canacugui 'iron nails.' A word which is placed second in these compounds may change its first letter; if it is $f$ it becomes $b$ or $p$, if it is $s$ it becomes $z$, if it is $c$ it becomes $g$, if it is $t_{\zeta}$ it becomes $z z$, if it is $x$ it becomes $j$; e.g., caribune, buppô, nigorizaqe, soragoto, qizzumari, and sorajeimon. See the dictionary.

\section{Pronouns}

In the Japanese language there are no derivative pronouns, such as meus,a,um, etc.; but the primative pronouns, such as mei, tui, etc., are used. These primitive forms do not have declensions for case, but rather use the particles which are common to both nouns and pronouns.

Certain particles (about which we will speak later) when added to a word indicate honor and thereby form a pronoun or substitute for it in such circumstances as pronouns would normally be used. Thus, if I say von fumi, when speaking to someone else, it is immediately understood that I am speaking about his letter and not mine; for if I were speaking about mine I would not say von fumi but only fumi, since the particle von, which indicates honor, signifies 'your letter.' This is also true for such particles as $m i$ which also attributes honor to the noun to which it is joined.

\section{First Person Pronouns-Ego, etc. ${ }^{22}$}

There are eight particles which signify 'I, mine, to me, etc.' They are vatacuxi, soregaxi, vare, mi, varera, midomo, midomora, vare. ${ }^{23}$ The first four indicate a degree of superiority on the part of those who use them. The others are more humble. Women use three other particles mizzucara, varava, and vagami which are not used by men. The people in the countryside use two others, vara [vora] and vorara, while priests

\footnotetext{
${ }^{22}$ The text reads De pronomine secundae personae ....

${ }^{23}$ This list, unquestionably derived from the Arte $(67 \mathrm{v})$, has been in several ways confounded. The $m i$ is out of order and the second vare is clearly in error. If we put aside the genitive forms from Rodriguez' list, the first four forms should be vare, varera, vatacuxi, and soregaxi. Rodriguez' second set consists of mi, midomo, and midomora. We would suggest that Collado meant to include ura, which is listed by Rodriguez as the genitive form vraga. I offer vatacuxi, soregaxi, vare, varera, mi, midomo, midomora, and ura as the intended list, with the order of $m i$ and varera reversed to accommodate the sentence which follows.
} 
when speaking of themselves use gusô, that is to say 'I, a worthless man of the cloth,' and old men when speaking of themselves use guró, 'I, a worthless and despicable old man.' The king (rex) says chin or maru which means 'I, the King.'

To form the plural of these pronouns the pluralizing particles domo or $r a$ are added; e.g., midomo ga maitta toqi 'when we went.' To indicate the difference between the cases, the endings about which we have spoken are suffixed.

\section{Second Person Pronouns-Tu, tui, tibi, etc. ${ }^{24}$}

There are many particles that form the second person pronoun. They are differentiated to indicate those persons deserving no honor and respect, those deserving some, moderate, great, or maximal honor and respect. In speaking to inferiors there are three particles used for 'you'; vare, vonore, and sochi. If me or mega is added as in vareme or varemega it means we very much despise the person being spoken to. If we speak to people who are on our own level, or just a little inferior, we use one of the three particles sonata, sonofó, or varesama. If we speak to a superior person, or someone on an equal level but with whom we must speak elegantly, we use one of the seven particles conata, qixo, qifó, gofen, qiden, conatasama, and sonatasama. When speaking to persons of high rank, if we place the name of their office before sama, it serves as a pronoun; e.g., Padresama gozare 'will the Father come.'

Conata, cochi, and conofó mean 'I, mine,' but in the distributive sense of 'from me, or what concerns me.' In the same way sochi, sonofó, and sonata mean 'you, from you, or what concerns you.'

The plurals are formed by adding the particles listed above to the pronouns according to the different degrees of honor. Vonore domo, varera, and sochira mean 'you' when speaking to inferiors. Vare tachi and sonata domo mean 'you' with persons of the same rank. Qifó tachi, vocatagata, and vono vono mean 'you' to persons requiring honor. The declension of these honorable expressions follows the declension of common particles.

\footnotetext{
24'The forms for the sccond person are derived from the Arte (68). Throughout this section the accent marks are quite erratic. In several places, for example, Collado has sónata and even sónatá.
} 


\section{Third Person Pronouns-Ille, illa, illud $d^{25}$}

The two particles care care and are are mean 'this (ille, illa, illud)' when speaking of inferior things. ${ }^{26}$ There are four particles; aitçu, aitçume, areme, and caiţume which mean 'this' when one wants to show disrespect for the things being spoken about. This idea is emphasized if one adds ga to those forms that end in me; e.g., aitçuga and aitçumega 'this humble man.' Cono means 'this (hic, haec, hoc),' sono means 'that (iste, ista, istud),' and ano means 'that (ille, illa, illud).' These words require a noun after them; e.g., cono fito 'this man' with cono mono having the same meaning but not being an honorific expression. Sono coto means 'that thing,' ano fito 'that person,' conata or conofó 'here,' sonata or sonofó 'there,' and anofó 'there, yonder.' Core means 'this (hic),' sore 'that (istud),' and are 'that (illud).' These forms are in the neuter gender and are not followed by nouns. Their plurals are corera, sorera, and arera, while the others follow the common rules. Cano means 'that which we have mentioned'; e.g., cano fito 'that person.' The pronoun 'a certain (quidam)' is made with the particle aru; e.g., aru fito 'a certain person,' aru tocoro $n i$ 'in a certain place.'

The pronoun 'each (unusquisque)' is formed with the particles men men and sore sore.

The pronoun 'each and every (universi \& singuli)' is formed with tare mo mina.

The pronoun 'anyone (quicumque)' is formed with tare nite mo, tare nite mo are, and tare nari tomo.

The particle tare mo, when placed before a negative, forms the pronoun 'no one, or nobody'; e.g., tare mo mairananda 'nobody went.' The particle nani taru coto nari tomo means 'whatever happens, or whichever thing happens.' The particle mei mei means 'to each, or everyone in particular.'

The particle goto makes the distributive pronoun meaning 'every.' This form is used after vocables which are proper to the Japanese language; i.e., iomi. The same results are achieved by placing the particle mai before vocables which come from the Chinese language; i.e.,

\footnotetext{
${ }^{25}$ In the material which follows Collado has brought together items from several sections of the Arte; for the interrogatives see $(65-65 \mathrm{v})$, the indefinites (66), and the demonstratives (68).

${ }^{20}$ These reduplicated forms are not derived from Rodriguez' description and are apparently misstatements of the forms care and are which would otherwise be missing.
} 
coie. For example, $f i$ means 'day,' and figoto ni means 'daily.' Nen is a Japanese borrowing from a Chinese word meaning 'year,' and mainen means 'every year, or all year.' The same result is obtained by the repetition of the noun; e.g., fito means 'person,' and fitibito means 'all the people, or many people,' $f i$ means 'day,' and fibi ni means 'all of the days, or every day.'

The indefinite pronoun 'some (aliqui)' is formed with niiotte; e.g., toqi niiotte 'some times,' fito niiotte 'some men.'

The pronoun 'the same (idem)' is formed with vonaji; e.g., vonaji tocoro cara 'from the same place.' The particle dôjen means the same thing but in the neuter; e.g., dôjen degozaru 'it is the same.' This word is used in reply to some one who has congratulated you, etc.

The pronoun 'himself (ipse)' is formed with the particles nuxi, sono $m i$, and vaga. The particle vareto $m i$ forms the pronoun 'himself (ipsemet)'; e.g., vareto mi ni ata vo nasu (96) 'he brings harm to himself,' mi vo vasurete; ta vo tasuquru 'he forgets himself and saves others.' The particle vatacuxi means 'a thing which belongs to oneself (re propria)'; e.g., vatacuxi no coto 'ones own thing,' vatacuxi ni ivareta 'he spoke for himself.'

The pronoun 'somebody (aliquis)' is made with the particles tare zo and taso; e.g., tare zo maittaraba 'if somebody were to come,' taso sacana ga aru ca tói ni iqe [...toi ...] 'let someone go and ask if there is food.'

The neuter pronoun 'something (aliquid)' is formed with the particles nan zo and nanica; e.g., nan zo ga araba cuvózu 'I would eat if there were something,' ima faia te ga jiiú ni gozaru fodo ni nanica caqi maraxô 'I would write something if I were to have my hands free, or untied.'

The interrogative 'who (quis)' is translated with the three particles tare, taga, and taso. The particles taga or tare no form the genitive; e.g., taga mono ca 'whose thing is this.' When someone comes to the door and knocks, he says mono mó. ${ }^{27}$ To this one responds taso, taga, or tare 'who is it?' Nani means 'what (quid)'; e.g., nani vo suru (17 ca or nani goto vo suru ca? 'what are you doing?' nani ni sore vo totte iqu ca? 'for what reason do you bring this to me?'

\footnotetext{
${ }^{27}$ An abbreviated form of monomósu; cf. Arte (139v).
} 


\section{Relative Pronouns}

The relative pronoun is formed by placing the noun, in connection with which there is a relative (relativum), after the verb; e.g., ten ni maximasu varera ga von voia 'Our Father who is in Heaven,' deta tocoro va 'the place from which he came out,' te ni sumi no tcuita fito (88) 'a man to whose hands ink is adhering.' If the sentence (oratio) requires a nominative before the verb it must be formed with one of the particles which indicate the nominative; ga, no, or iori. For example, vatacuxi ga caita fumi 'the letter which I wrote,' conata no vôxerareta coto 'the thing which Your Lordship says.' The third particle, iori, is used when there is movement in the sentence; e.g., Deus iori ataie cudasareta gracia 'the grace which God provided, or gave,' ano tocoro ni amata no qió atta vo torareta $(87 \mathrm{v}$.) 'he brought what many books there were in that place.' When two sentences containing a relation come together the first is placed second by general rule, ${ }^{28}$ and the second uses either a past, present, or future particle according to what is required by the sense of the sentence; e.g., qesa Oracio vo móxita qió ga tçucuie no uie ni aru vo motte coi 'bring the book which is on the desk (sedila) at which I said my prayers this morning.' In this sentence qió $g a$, which is the first relative, comes after the verb móxita; and the $v o$ which stands for the second relative comes after the verb aru. When we want to be more specific about that of which we are speaking we place the particle tocoro no between the thing itself and the verb; e.g., vare to doxin xita tocoro no mono domo va mina buguen ni natta 'all those who agreed with me became rich.' Sometimes the relative, because of the difficulty in understanding it, is expressed by expositions (per exponentes). Thus, in place of ima corosareta Pedro no co va sonata no chijn gia which means 'the son of Peter who has just been killed was your friend,' we say ima Pedro corosareta sono co va sonata no chijn de gozaru.

Sometimes they join two particles, as determined by the case, and form a kind of relative pronoun which is placed before the relative; e.g., sono tocoro de no dancó 'the consultation at that place,' Marsella ie no fune 'the ship to Marseille,' maire to no móxi goto dearu $[\ldots$ gia $]$ 'it is said that I should go.'

\footnotetext{
${ }^{28}$ Collado is here speaking with reference to the normal order in Latin.
} 
Mairu mai to no dancó ni qivamatta 'it was resolved that he not go,' maitte nochi no dancó 'the consultation he arrived after,' varambe cara no catagui 'a custom from youth,' xô tame no chôgui gia (22) 'this is the plan (ars) according to which it will be done,' anofito no vo toró 'I shall take what belongs to that man.' This ends the note on relative pronouns.

\section{The Formation of the Verb and Its Conjugation ${ }^{29}$}

The verbs in Japanese have no number or person. These distinctions are indicated instead by the particles used in the formation of the plurals and in the declensions. There are three affirmative conjugations and the same number of negative.

The root (radix) of the verb does not by itself indicate tense. For this reason it is necessary to conjugate the verb in order to show the tenses.

All the verbs of the first conjugation ${ }^{30}$ end in $e$. Those ending in $g i$ or $j i$, together with $x i$ and maraxi, although they end in $i$, are also in the first conjugation. If the root ends in $d e$ or gi, the present form is made by changing them to zzuru; e.g., fagi forms its present in fazzuru and means 'to blush,' de becomes zzuru and means 'to leave.' If the root ends in $j e$ or $j i$ it changes in the present to zuru; e.g., maje:mazuru 'to mix,' anji:anzuru 'to consider.' If they end in $x e$ they change to suru; e.g., avaxe:avasuru 'to join.' $X i$ and maraxi, which (as we have said) are in the first conjugation, ${ }^{31}$ change $x i$ to suru; e.g., xi:suru 'to do,' maraxi:marasuru which also means 'to do.' If the root ends in te it changes to tçuru; e.g., sodate:sodatçuru 'to nourish, or support.' The remaining roots which end in $e$ change, in their separate ways, the $e$ to uru; e.g., ague:aguru 'to offer,' nigue:niguru 'to run away.'

There are certain verbal preterits which have present tense meanings. They are those which are passive in form but active in meaning; e.g., cocoroieta 'to understand,' qicoieta 'to hear,' voboieta 'to remember,' qiqiieta 'to understand,' zonjita 'to know,' and there may

\footnotetext{
${ }^{29}$ The treatment of the verbal system by Collado follows in a general way the Arte $(6 \mathrm{v}-54 \mathrm{v})$. In the material that follows specific references will be made when a comparison of the two works is suggested.

${ }_{30}$ The text has secundae coniugationis. This error, which is repeated throughout the text, is not present in the Spanish manuscript.

${ }_{31}$ The text again has secundae coniugationis.
} 
be many others. The verbs which follow belong to the first conjugation even though their roots do not end as previously stated. ${ }^{32}$ If the present tense of these forms does not change the $i$ to uru they are exceptional; e.g., abi,uru 'to wash oneself,' fotobi,uru 'to become soft,' focorobi,uru 'to become unstitched,' cabi [cabi,uru] 'to be moldy, ${ }^{33}$ sabi [sabi,uru] 'to rust,' deqi [deqi,uru] 'to be finished, or ended,' cuchi:cutçuru 'to rot,' michi:mitçuru 'to be filled in by the sea,' ini,uru 'to leave,' nobi:nobiru or noburu 'to be spread out,' tçuqi,uru 'to be used,' vori:uru 'to descend from above,' xij:xijru 'to invite to dine, by compulsion,' ni:niru 'to resemble,' mochij:mochiiuru 'to evaluate,' ni:niru 'to cook,' mi:miru 'to look at,' cori,uru 'to correct,' vochi: votçuru 'to fall,' i:iru 'to exist, or be present,' fugui,uru 'to pass, as time passes,' vabi,uru 'to beg for mercy,' carabi,uru 'to become dry,' iqi:iquru 'to live,' fi:firu 'to become dry,' qi:quru 'to come,' qi:qiru 'to dress oneself,' voqi,uru 'to get out of bed.' The following four verbs have irregular, as well as regular, present tenses; ${ }^{35}$ ataie has atóru 'to give,' vaqimaie has vaqimóru 'to discriminate,' tonaie has tonóru 'to bless,' sonaie has sonóru 'to place in a high position.'

\section{The Preterit, Perfect, Imperfect, and Pluperfect}

In Japanese there is no imperfect. In its place the perfect is used. The perfect is formed in two ways. The first is by suffixing $t a$ to the root of a verb; e.g., agueta is the preterit of the verb ague,uru 'to offer.' The second is by suffixing te to the root and to that adding gozari, $u$ or ari, $u$ which is then conjugated in the present or the preterit of the second conjugation; e.g., aguete gozaru or aguete gozatta, or aguete aru or aguete atta 'offered, or had offered.' If the particle fáia [faia] is placed before the verb the expression is strengthened; e.g., fáia aguete gozatta [faia...] 'I had already offered it.' When the verb ari, $u$ is suffixed to the perfect it is not as elegant a way of speaking as

\footnotetext{
${ }^{32}$ This list covering the Kami-ichidan and Kami-nidan verbs is derived from a similarly defined sub-group of the first conjugation in the Arte (28). Since the verbs cabi, sabi, and deq $i$ are in no way indicated as extraordinary in Rodriguez' presentation, I have amended the text to include their present tense form.

${ }^{33}$ The text reads for this gloss fucore afficior. The proper word is mucore 'mould,' with the literal translation being 'I am affected by mould.'

${ }^{34}$ The Dictionarium has this verb listed as kami-nidan, xij, uru, and therefore not exceptional.

${ }^{35}$ Cf. Arte (7) where a similar list is presented.
} 
when gozari, $u$ is used. Therefore when speaking one must be careful about what one says, or in front of whom one speaks, so as to give each person the honor he deserves.

\section{The Future of the First Conjugation}

If the root of the verb ends in te this syllable is changed to teô or chô to form the future; e.g., tate,uru will become tateô or tachô 'I shall build. ${ }^{36}$ If the root ends in $j i$ the future is formed by changing $j i$ to $j \hat{o}$; e.g., xenji becomes xenjô 'I shall prepare, or brew, the medicine.' If the root ends in $x e[x i]$ it changes to $x \hat{o}$; e.g., $x i$ becomes $x \hat{o}$, and maraxi becomes maraxô 'I shall do." If it ends in ie it is changed to io [iô]; e.g., voxiie becomes voxiio [vaxiiô] 'I shall teach.' The remaining roots ending in $e$ suffix the particles $\hat{o}, \hat{o} z u$, or $\hat{o} z u r u$; e.g., agueô, agueôzu, or agueôzuru 'I shall offer.' These endings are used for the first conjugation $^{37}$ even when the roots end in $i$; e.g., deqiôzu 'I shall be finished.'

The future is also formed by taking the syllable $n u$ from the negative present (see below) and putting in its place the particle baia. Thus, by taking $n u$ away from aguen $u$ and putting in its place baia, we obtain aguebaia 'I will offer.' For minu if you take away the $n u$ and put in its place baia it will become mibaia 'I will see, or behold.'

The future perfect is formed by suffixing the particles te arózu or tarózu to the root; e.g., aguete arózu or aguetarózu 'I shall already have offered.' The same results are obtained if faia is placed before the simple future; e.g., faia agueôzu.

\section{The Imperative of the First Conjugation}

The imperative of the first conjugation is formed with the root of the verb alone, or with the addition of the particle io; e.g., ague or ague io 'offer!'38 The future of the imperative is the future absolute agueô or agueôzu. This is a more elegant and polite way of speaking than giving a command with the regular imperative. The imperative is also formed by taking the $n u$ from the negative present (see below) and

\footnotetext{
${ }^{36}$ For the source of Collado's description of the future tense cf. Arte (7v).

${ }^{37}$ The text reads secundae coniugationis.

${ }^{38}$ Rodriguez more correctly has this rule as the root plus $i$ or yo; e.g., aguei or agueyo. The form ague $i$ is used by Collado in the construction of the optative below.
} 
putting in its place the particle sai. Thus, if one takes the $n u$ from aguenu and replaces it with sai it becomes ague sai which means 'offer!' If the particle tai is placed after the root there is formed a kind of future or optative by which the wish of the speaker is expressed. It is therefore an elegant imperative; thus mizzu fitotçu nomitai 'I would like to have a drink of water' is the same as 'give me some water to drink.' When a relative [clause] concerns a precept, rule, admonition, or prohibition the imperative is expressed word for word in whatever the conjugation, affirmative or negative; e.g., Christiani naru na to no xógun no fatto ga aru [Christian ni ...] 'it is the law of the Shōgan (imperator) that no one should become a Christian,' Padre core vo

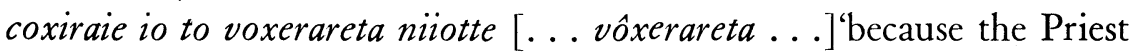
told me to do it.'

\section{The Optative of the First Conjugation}

The optative, both present and future, is the present tense of the imperative with the particles negavacu va or avare placed before it and the particles gana or caxi placed after it. Sometimes it is formed by adding the particle gana without any prefix; e.g., negavacu va ague io caxi? or avare aguei gana $a^{39}$ 'would that you were to offer?' avare icanaru tengu, bangue mono nari tomo vare vo totte, fiie no iama ni noboxe io caxi! (15v) ${ }^{40}$ 'Oh! if there were some one, either devil or soothsayer, who could make me ascend the mountain called Hie.' The particle gana when it is placed after a noun indicates a wish for the thing specified by the noun; e.g., saqe gana 'oh! sake'; and if (22 one is asked if he would like something to drink, the answer is nani gana 'would that I had some.'

The perfect of the optative is the second form of the future followed by the particle mono vo!; e.g., niqueozu mono vo! [nigueôzu ...] 'would that I had fled!' The same is achieved by niguetaraba iocaró mono vo. Sometimes they say only niguetaró va or niguete aró ni va iocaró mono vo.

${ }^{39}$ This form is correct but does not follow his rule for the formation of the imperative (sec note 38 ).

${ }^{40}$ Rodriguez has baquemono 'evil spirit' and the Spanish manuscript baqemono, rather than banguemono 'soothsayer.' 


\section{The Subjunctive of the First Affirmative Conjugation}

The present tense of the subjunctive is formed by changing the $u$ in which the present indicative ends to $e b a$; e.g., aguru becomes agureba 'since I offer.' It is also formed from the present by adding $n i$, de, vo, or $v a$ to the particle tocoro according to the case requirements of the verb that follows, with the first verb being controlled by the noun; e.g., aru toqi Pedro chinsui xite iraruru tocoro ie fito ga qite (16v) ${ }^{41}$ 'since a certain man came to the place where Peter was when he was drunk,' nhóbó ni tachi vacarete iru tocoro $n i(16 \mathrm{v})^{42}$ 'since they were separated and divorced,' có aru tocoro $n i$ 'since things are this way,' ioso ie zzuru tocoro va fito ni corosareta $(16 \mathrm{v})^{43}$ 'when he went outside, he was killed by someone,' go misa vo asobaruru tocoro vo uchi coroita $(121)^{44}$ 'he killed him while he was celebrating mass.' This is a general rule which applies to all conjugations.

The perfect and the pluperfect of the subjunctive are formed from these same tenses in the indicative with the addition of the particle reba; e.g., agueta reba 'since he had offered.' It is also formed by taking away gozaru from the preterit pluperfect and putting in its place atta reba or atta; but, when atta is used, the particles $n i, v o, v a$, or ie must be added according to the requirements of the following verb, just as with tocoro in the present tenses; e.g., aguete atta reba or aguete atta ni, $v o, v a$, or ie 'since I had already offered it.'

The future of the subjunctive is formed by adding the particle toq $i$ to the future indicative; e.g., agueô toqi 'since he would offer it later.'

The pluperfect subjunctive, with all the expressions (vox) which signify that which comes after a completed action, is formed by placing cara, nochi, or igo after the pluperfect indicative, minus gozaru;

${ }^{41}$ Extracted from Rodriguez' version of a sentence in the Amakusa edition of Esop's Fables (p. 417). The original reads, Arutoqi Xantho chinsui xite yrarturu tocoroye, fitoga qite daicaino vxiuouo fitocuchino nomi ţ̧ucusaruru michiga arócato tôni,... . 'One time when Xantho [Esop's master] was drunk, a man came and asked if there was a way to drink all the waters of the ocean in one swallow. . . it is abbreviated by Collado in such a way as to obscure the construction.

${ }^{42}$ Also apparently extracted from the Esopo (p. 477). The original has, . . riobboni tachiuacarete yru tocoroni, qiţ̧unega yosocara coreuo mite, futaţ̧uno nacani vocareta fitţu jiuo totte curóta, 'when they [two lions] had gone their separate ways, the fox, sceing this from afar, took the sheep which had been between the two of them and ate it.' By changing rióbó to nhóbó Collado created a less than satisfactory example.

${ }^{43}$ Modeled on Iyeuo idzuru tocorouo cubiuo quiri votoita 'when he went outside his head was cut off.'

" Modeled on Missauo asobasaruru tocoroye vôjei faxe atçumatta 'when mass was being celebrated, many came running and gathered around.' 
e.g., aguete cara, nochi, or igo, mairó 'I shall leave after he has offered it.' This is like aguetaró toki mairó 'I shall leave after he has already offered it.' Agueôzuru ni or agueôzuru tocoro ni means 'since he was already prepared to offer it.' Agueôzuru coto no saqi ni means 'a little while before he offered it.'

The present tense of the permissive subjunctive is formed in two ways. The first is by changing the $u$ of the present indicative to edomo; e.g., aguredomo 'although I could offer it.'

The preterit of the permissive subjunctive is formed by adding redomo to the preterit indicative; e.g., agueta redomo 'although he had offered it.' The future permissive is formed by adding redomo to the second form of the future indicative; e.g., agueôzu redomo 'although he would be able to offer it.' The second form of the permissive subjunctive is formed by adding the particle tomo to the present indicative; e.g., aguru tomo 'although he could offer it.' The particles mamaio or madeio may also be added to the present tense; e.g., sore vo voxiiuru mamaio or sore vo voxiiuru madeio 'although he could teach this.'

The preterit of the second permissive is formed by suffixing ritomo to the preterit indicative; e.g., agueta ritomo 'although he had offered it.' The same meaning is achieved by adding the particles mamaio or madeio to the preterit indicative; e.g., agueta mamaio or agueta madeio; or by adding tote to the preterit subjunctive; e.g., aguetareba tote.

The future permissive is formed by adding tomo to the second form of the future indicative; e.g., agueozu tomo [agueôzu tomo 'although he would offer it']. It is also formed by adding mamaio or madeio to the same future form. If the particle tato $i$ is placed before the forms of the permissive subjunctive great strength is added to the sentence; e.g., tatoi vôxeraruru tomo 'even though you may state this.' The same meaning is obtained by removing the verbs gozaru or aru from the pluperfect indicative and replacing it with the particle mo; e.g., aguete mo 'although he may offer it.' The same mo when placed after the present indicative gives the same meaning; e.g., doco de qiqi marasuru mo, sono sata va mósanu 'although he hears that everywhere, he does not pay any attention.' The same meaning is obtained by the sentences ague mo xeio caxi?, aguete mo xô madeio, and nanto mo ague caxi? 
agueta ni saxerarei, or agueo ni saxerarei $[$ agueô...] have the meanings of 'although he could have offered, although he could offer, or although he would offer'; or one might say 'let us offer' or 'let us give.'

\section{The Infinitive}

The present infinitive is formed by adding coto or to to the present indicative; e.g., aguru coto or aguru to 'to offer.'

The preterit infinitive is formed by adding the same particles to the preterit indicative; e.g., agueta coto or agueta to 'to have offered.' The future infinitive is formed by adding the same particles to the future indicative; e.g., agueô coto or agueô to 'to be about to offer.' The same meaning is obtained by adding ióni to the present, preterit, or future indicative; e.g., nai nai guioi ni caqerareô ióni va vare mo zonzuru fitobito mo zonjita (22v) 'I think and others believed me to have been favored by you with many benefits,' qeccu vare ni voxiie marasuru ióni gozaru $(117 \mathrm{v})$ 'he is truly able to teach me,' agueta ióni gozaru 'he is said to have offered it.'

To ask or answer a question the infinitive is often subordinate to the verb which follows; e.g., nhóbógata ni vochita coto ga atta ca? 'did you fall into the sin of adultery with this woman? is this what happened?' etc. All the tenses of the infinitive are used in the same way.

Sometimes the preterit infinitive is replaced by the pluperfect with gozaru or aru removed; e.g., Deus no minori vo firomete iocaró 'it is good to spread the Gospel.' Sometimes the present or preterit indicative plus $g a$ replaces the present or preterit of the infinitive; e.g., sore vo vôxeraruru ga varú gozaró 'it will be bad to say that,' maitta ga maxi gia (21) 'it is better to have come, or it was better to come.'

When the substantive verb follows the infinitive, the particle coto is not required; e.g., cosacazzuqi de va saqe vo nomu devanai (23) 'to drink sake from a small glass is not to drink sake,' core coso caqu de gozare 'this we are able to say, or better, write,' caqu de gozatte coso 'this is not the way for it to be written,' sore va aguru devanai 'that is not to offer it.' Some of these examples are taken from other conjugations but the general rule applies to all. The idea of the in-

\footnotetext{
${ }^{45}$ Apparently modelled after Arte (20v) nantomo voxiare caxi 'whatever you say,' with the imperative formation again confounded.
} 
finitive is also obtained by the following means of expression; ague va, aguredomo 'although I offered, or even if I made it so that it was offered.' Because this is a general rule for all the conjugations, they also say qiqi va tçucamatçure domo gatten xenu 'although I have listened, or done everything necessary to hear; I still don't understand.' They also say aguru vo motte 'by offering, or with the fact that he is to offer,' aguru iori 'from the fact that he is to offer', aguru nitçuite 'about the fact that he is to offer.'

The gerund in $D i$ is the present or future indicative followed by the particle jibun, or less frequently some other particle meaning 'time'; e.g., aguru jibun 'the time for offering,' agueô ni qivamatta 'he made the decision that it be offered,' niguru jibun gia 'it is time to flee," corosareôzuru ni aisadamatte arǒzu (13) 'it will have been decided that he will be killed, or will have to be killed.'

The gerund in $D o$ is formed in two ways. The first is by adding the particles ni or tote to the present indicative; e.g., aguruni or agurutote iurusareta 'I was freed by it being offered.' The second way is by removing the verb gozaru from the pluperfect; e.g., aguete cutabireta 'I became tired by offering, or raising up,' that is to say, 'from the action of presenting, or raising up, I suffered the result of becoming tired.' There is also another elegant, and frequently used, way to form the gerund in $D o$. It is done by placing the root of the verb in front of another verb making a compound; e.g., fiqi iosuru 'to approach, pulling.' The roots which are used in this way do not change with respect to their function. The gerund in $D o$ is also used to express purpose taixó to xite 'since he was a commander $(d u x)$, or was fulfilling the function of a commander,' von rei to xite 'giving thanks,' rótai nomi ni xite 'since he was an old man,' tçucai xite ivaruru 'he said it as a messenger.'

The gerund in Dum is formed by adding the particles tame or tote to the present or future indicative; e.g., aguru tame or agueo tote [agueô tote] 'in order to offer.' The same meaning is obtained by aguru ni fatto ga aru 'there is a law about offering,' unless this should be considered a gerund in ni $[D i]$.

The supine in Tum is formed in two ways. The first is by adding $n i$ to the root. The second is by adding tameni to the present indicative; 
e.g., tazzune ni maitta or tazzunuru tameni maitta 'I came in order to obtain it.'

The supine in $T u$ is the root of the verb alone. To obtain the same meaning they also use mósu ni voiobanu it is not necessary to speak.'

The present, preterit, and future participles are formed by adding the particles fito or mono to the indicative. When fito is used the result is a more honorable way of speaking; e.g., aguru fito or aguru mono 'he who offers,' agueta fito 'he who offered,' agueô mono 'he who will offer,' Buppôgacu suru tomogara ni voite va (73v) 'as for those who devote themselves to the study of the laws of idolatry,' von vo xiru vo fito to va iúzo; von vo xiranu voba chicuxó to coso iie (96v). In this last sentence the $v o$ takes the place of the participle, and the sentence therefore means 'those who know kindness (beneficia) are correctly called men; those who do not know it are truly called beasts.' This is a general rule for all the conjugations and therefore the example contains a verb from the second conjugation. The participle is also made by adding te ['hand'] to the root of the verb; e.g., aguete 'one who offers.'

\section{The First Negative Conjugation}

The negative root is formed by adding $z u$ to the affirmative root; e.g., aguezu.

The present tense is formed with $n u$ instead of $z u$; e.g., aguenu' 'I do not offer.' This is a general rule no matter how the root ends. The only exceptions are $x i$ and maraxi which form the negative present in $x e n u$ and maraxenu 'I do not do.' The roots that end in $j i$ change the $j i$ to $j e$ and then suffix the particle $n u$ to the present; e.g., zonji in the negative present becomes zonienu [zonjenu] 'I do not know.' In some areas of Japan they form the negative by removing the final $u$ from the negative root and adding ari,u, which is then conjugated according to the required tense; e.g., aguezaru 'I do not offer, aguezatta 'I did not offer,' aguezatta reba 'since I did not offer.' They also say aguezu xite 'by not offering. ${ }^{46}$

\footnotetext{
${ }^{48}$ Rodriguez (25v) specifies the location of this usage as Chügoku, Bungo, Hakata, and other Ximo districts.
} 
The negative of the preterit is formed in like manner by adding the particle nanda instead of $n u$; e.g., aguenanda 'I did not offer', zonjenanda 'I did not know,' vorinanda 'I did not decend.'

The pluperfect is formed by changing the last $a$ of the preterit to $e$ and adding the verb gozaru in the present and gozatta in the preterit; e.g., aguenande gozaru or aguenande gozatta 'I have not offered.' It is also formed by adding ide gozaru or ide gozatta instead of nande gozaru; e.g., agueide gozaru or agueide gozatta 'I had not offered,' zonzeide gozaru [zonjeide ... .] 'I7 'I had not known,' vochiide gozatta 'I had not fallen.'

The negative future is formed by adding mai or maji to the affirmative root or the affirmative present tense; e.g., ague mai or aguru maji 'you will not offer.'

The imperative is formed by placing $n a$ after the present indicative; aguru na'do not offer.'

It is also formed by placing $n a$ before the root and so after it; e.g., na ague so 'do not offer.'

It is also formed by placing $n a$ after the root; e.g., ague na 'do not offer,' mixe na 'do not show,' mesare na 'do not do.' The roots which end in $x i$ or $j i$, but are in the first conjugation, ${ }^{48}$ change the $i$ to $e$ to form the negative imperative; e.g., só xe na or só maraxe na 'do not do that,' só zonze na [só zonje na] 'do not think that.'

The optative is formed by placing negavacuva or avare before the negative imperative and placing caxi or gana after it; e.g., avare aguru na caxi 'oh! if only you would not offer,' and negavacuva na ague so gana with the same meaning.

The preterit of the optative is formed by placing mono vo after the negative future; e.g., aguru mai mono vo 'oh! if only you would not have offered.'

The negative subjunctive is formed by changing the $u$ which ends the negative present to $e b a$; e.g., agueneba 'since he did not offer.'

The preterit of the subjunctive is formed by adding reba to the negative preterit of the indicative; e.g., aguenanda reba 'since he had not offered.'

\footnotetext{
${ }^{47}$ This example, together with so zonze na below, reflects the loss of a distinction between $z$ and $j$ which was taking place during this period.

${ }^{48}$ The text has secundae coniugationis.
} 
The future of the subjunctive is formed by adding qereba to the negative future; e.g., niguru mai qereba 'since he is not going to escape.'

The permissive subjunctive is formed by adding domo to the negative present after changing the final $u$ of the verb to $e$; e.g., aguenedomo 'although he cannot offer.' They also say, and this usage is preferred, aguenaidemo or agueidemo 'even if he not offer. ${ }^{49}$

The preterit of the permissive subjunctive is formed by placing redomo after the negative preterit; e.g., aguenanda redomo 'although he had not offered.' Aguenaidemo or agueidemo 'although he would not be allowed to offer,' is also said.

The permissive future is formed by adding qeredomo to the negative future; e.g., aguru mai qeredomo 'although he is not going to be allowed to offer.'

Another way of forming the permissive subjunctive is to place the particle tomo after the negative root; e.g., aguezu tomo 'although he is not going to be able to offer.' It is also formed by placing tote after the [negative] present subjunctive; e.g., agueneba tote. A third way is to add mamaio or madeio to the negative present; e.g., aguenu mamaio or aguenu madeio 'although he cannot offer.'

The preterit is formed by placing ritomo after the negative preterit; e.g., aguenanda ritomo 'although he had not offered.' It is also formed by placing tote after the negative preterit of the subjunctive; e.g., aguenanda reba tote, or better, aguenaidemo or agueidemo 'although he does not offer, or had not offered.'

The future is formed by placing tomo after the negative future; e.g., aguemai tomo 'although he is not going to offer,' vochiidemo 'although he will not fall.'

The present, preterit, and future infinitives are the present, preterit, and future negative indicative present tenses followed by coto or to; e.g., aguenu coto 'not to offer,' aguenanda coto 'not to have offered,' aguru mai coto 'not to be going to offer.'

Sometimes they use the negative present instead of the preterit in all the conjugations; e.g., mi maraxenu 'I did not see.'

\footnotetext{
${ }^{49}$ The Arte (27) records here aguenedomo, aguenuto móxedomo, aguezutomo, aguenebatote, and agueidemo. Neither aguenaidemo nor the participle aguenaide, below, are found in the Arte, although they are attested to elsewhere. Cf. Yuzawa Kōkichirō, Edo kotoba no kenkyyu (Tokyo, 1954), p. 626.
} 
The negative gerund in $D i$ is the same as the negative present or future; e.g., aguenu or aguru mai 'of not offering.'

The gerund in $D o$ is formed by placing $n i$ after the negative root or the negative present tense; e.g., aguezuni or aguenuni 'by not offering.' The same meaning is obtained with agueide, aguenaide or aguezu xite.

The gerund in Dum is formed by placing tote or tame after the [negative] present or future of the indicative; e.g., aguenu tame or aguru mai tote 'in order not to offer.'

The present, preterit, and future participles are formed by adding fito or mono to the negative of the present, preterit, and future indicatives; e.g., aguenu fito 'he who is not offering,' aguenanda mono 'he who did not offer,' aguru mai mono 'he who will not offer,' aguenaide cara or agueide nochi 'after he had not offered, after they did not offer, or after it was not offered.'

\section{The Second Affirmative Conjugation}

All the roots of the second conjugation end in $i$ and form their present tense by changing $i$ to $u$; e.g., iomi:iomu 'I read.' If the root ends in $c h i$ it changes its ending to tçu e.g., machi:maţ̧u 'I wait.' If the root ends in $x i$ it changes to su; e.g., coroxi:corosu 'I kill.'

For the preterit, if the root ends in ami it changes to óda; e.g., cami:códa 'I ate, or chewed.' If it ends in ebi or emi it changes to eôda; e.g., saqebi:saqeôda 'I am injured,' sonemi:soneoda [soneôda] 'I envied, or I had envy.' If it ends in obi or omi it changes to $\hat{o} d a$; e.g., corobi:corôda 'he fell," comi:côda 'it enclosed itself.' If it ends in umi it changes to unda [unda]; e.g., casumi:casunda 'it is cloudy.' The same change is made for roots ending in imi; e.g., canaximi:canaxúnda [canaxunda] 'he became sad.' If it ends in gui it changes to ida; e.g., fegui:feida 'it is divided.' Xini,uru has the preterit xinda 'he is dead,' and ini:uru has the preterit inda 'he left.' While in this respect they [xini and ini] are in the second conjugation, in the other tenses they are in the first. A root ending in chi or $r i$ changes in the preterit to tta; e.g., mochi:motçu in the preterit becomes motta 'he received,' chiri,u:chitta 'it is scattered.' Those which end in $x i$ or $q i$ change to ita; e.g., coroxi,u:coroita 'he killed,' qiqi,u:quita 'he heard,' xiqi,u:xiita 'he stretched it out.' 
The future is formed by changing the $i$ in which the root ends to $o$, ózu, ózuru; e.g., iomó, iomózu, or iomózuru 'you will read.' If the root ends in chi it changes to tó; e.g., machi:mató 'I shall wait.' A root ending in $x i$ changes to só; e.g., móxi,u:mósó 'I shall say, or speak.'

The imperative is formed by changing the $i$ in which the root ends to $e$; e.g., iomi:iome 'read! or may you read.' If the root ends in chi it changes to te; e.g., machi:mate 'wait!' The imperative is also formed by changing the $n u$ in which the negative present ends to $a i$; if you remove the $n u$ from iomanu and replace it with ai it gives you yomai 'read!" ${ }^{50}$ This is a common rule for the third conjugation, but this imperative is used only when addressing inferiors.

The future of the imperative is the future absolute; e.g., iomó 'you will read.' This is used when addressing very low people.

The remaining tenses of the optative, subjunctive, gerund, and infinitive are formed in the same way and with the same particles as are used for each in the first conjugation.

\section{The Second Negative Conjugation}

The root of the negative second conjugation is made by changing $i$, in which the affirmative root ends, to $a z u$; e.g., iomi:iomazu 'not reading.'

If the root ends in chi the present tense is formed by changing it to tanu; e.g., machi:matanu 'I do not wait.' If it ends in $x i$ it changes to sanu; e.g., coroxi:corosanu 'I do not kill.' If they end in any other way change $i$ to anu; e.g., corobi:corobanu 'I do not fall.'

The preterit is formed by changing the $n u$ of the present tense to nanda; e.g., corobanu:corobananda 'I did not fall,' iomananda 'I did not read.' The other tenses are formed in the same way as the negative first conjugation.

\section{The Third Affirmative Conjugation}

The roots of the third conjugation end in $a i$, oi, or $u i$. Those ending in ai change to ó to form the present; e.g., narai:naró 'I learn.' Those

\footnotetext{
${ }^{50}$ This rule, derived from Rodriguez (Arte, 29), is misformulated by Collado. Rodriguez' rule is correct; change the $n u$ of the negative present to $i$. It is formulated correctly for the third conjugation, below.
} 
ending in oi change to $\hat{o}$; e.g., vomi:vomô 'I think.' Those ending in $u i$ change to $u$; e.g., cui:cú 'I eat.'

The preterit is formed by adding $t a$ to the present tense; e.g., naróta 'I learned,' vomôta 'I thought,' cúta 'I ate.'

The pluperfect is formed by changing the final $a$ of the preterit to $e$ and adding the verb gozaru in the present and gozatta in the past, in the same way as we have described for the first conjugation; e.g., naróte gozaru or naróte gozatta 'I have already learned.'

The future is formed by changing the final $i$ of the root to vó, vózu, or vózuru; e.g., naravó, naravózu, or naravózuru 'I shall learn.' If the root ends in oi it is changed to vô, vôzu, or vôzuru [vó, vózu, (31 or vózuru]; e.g., vomoi:vomouô, vomovozu, or vomovôzuru [vomoi: vomovó, vomovǒzu, or vomovózuru] 'I shall think. ${ }^{.51}$

The imperative is formed by placing $e$ after the root; e.g., naraie 'learn!' toie 'ask!' cuie 'eat!'52 It is also formed by removing the syllable $n u$ from the negative present tense and replacing it with the letter $i$; e.g., naravai 'learn!' tovai 'ask!' cuvai 'eat!' This form is used when addressing inferiors, as are those of the other conjugations.

\section{The Third Negative Conjugation}

The root of the third negative conjugation is formed by changing the $i$ of the affirmative root to vazu; e.g., naravazu, tovazu, and cuvazu. The present tense is formed by changing the $i$ to vanu; e.g., naravanu 'I do not learn,' tovanu 'I do not ask,' cuvanu 'I do not eat.'

The preterit is formed by changing the $i$ of the root to vananda; e.g., naravananda 'I did not learn,' tovananda 'I did not ask,' cuvananda 'I did not eat.'

The pluperfect is formed by changing the final $a$ of the preterit to $e$ and adding the verb gozaru or gozatta; e.g., cuvanande gozatta 'I had

\footnotetext{
"rollado's rule clearly confuses the formulation of the present with that of the future. Significantly in the Arte Rodriguez never refers to the future forms of any verb other than his model narai. If Collado had had access to the Arte Breve he would have found (41) the following principal parts for vomoi; vomoi, vomô, vomôta, vomovó, vomoye. The only other use in the Ars Grammaticae of this form is on page 62 where Collado has the incorrect form vomov'ô. The manuscript does not record this form.

${ }_{52}$ Although Collado's transcription permits this rule to yield the appropriate forms, it obscures the fact that the final $i$ of the root is a vowel, while the $i$ of the imperative is a semivowel. Rodriguez' transcription better reflects the phonological facts; naraye, vomoye, and cuye.
} 
not eaten,' or naravanande gozaru 'I had not learned.' The remaining forms are like the other conjugations. ${ }^{53}$

If the substantive verb is placed after the gerund in $D o$ for all the affirmative and negative conjugations, it means that the action signified by the gerund is or is not done; e.g., aguete aró 'it will already be offered,' cono qió ga caite gozaranu 'this book is not written,' agueide arózu 'he will not yet have offered.' The substantive verbs are gozaru: gozaranu, voru:vori nai, dea or gia:devanai, aru:aranu or gozaranu, voru:voranu, and each of these verbs follows the general rules for its conjugation. ${ }^{54}$

If the substantive verb from any of the conjugations is placed after the infinitive form it means that whatever is signified by the infinitive is, was, or will be; or the negative thereof; e.g., aguru coto aró 'it will be that he offers,' that is to say 'he will offer,' naróta coto gozaru mai 'he will not learn.' All these substantive verbs are conjugated in the second conjugation to which they belong by virtue of the fact that their roots end in $i$; ari,u:gozari,u.

\section{The Conjugation of the Negative Substantive Verb}

The negative substantive verb is nai, gozanai, or vori nai which means 'not to be.' Its root is naqu, gozanaqu, or vori naqu.

The preterit is formed by changing the $i$ in which the present tense ends to $c$ and then adding the preterit of ari, $u$ which is atta; e.g., nacatta or gozanacatta 'he was not.' The other tenses are conjugated, as is ari,u, in the second conjugation.

The imperative is nacare, nanaiso, or nai na 'be not!'

\footnotetext{
${ }^{53}$ This completes Collado's treatment of the third negative conjugation. The two paragraphs which follow are part of his treatment of the substantive verb. There is no section heading for the affirmative substantive verb; and clearly a portion of the text has been deleted. The Spanish manuscript (cf. Ōtsuka's 1957 edition, p. 45) includes a new section which begins by recording the following substantive verb forms; ari:aru, gozari:gozaru, i:iru, and vori:voru.

${ }^{54}$ Collado's presentation of the substantive verbs is obscure. The text reads: Verba verò substantiua sunt, gozaru, gozaranu, voru, uori nai, dea vel gia: deuanai, aru:aranu, vel, gozaranu uoru ùôrinai, $\mathcal{E} \ldots .$. The translation attempts to punctuate the list to reflect the contrast between affirmative and negative forms. The main confusion is the apparent effort to contrast voru and vorinai. Vort (glossed by the supplement of the Vocabulario as estar, and used in the Dictionarizum as the gloss for existo, etc.) is not used by Rodriguez in the Arte. Vorinai (unglossed in the dictionaries) is clearly defined by Rodriguez as the negative of the polite verb voriaru, which is derived by him from von iritaru (Arte, 165v). Possibly Collado had intended to contrast voru with voranu and voriaru with vorinai but confounded the two pairs and then repeated his error at the end of the list; or again he may, in the absence of Rodriguez' guidance, have simply misunderstood the matter. Putting the alternative forms aside, the list should read gozaru:gozaranu, vori aru:vori nai, gia:devanai, aru:aranu, and voru:voranu. Collado's treatment is patterned only loosely after the Arte $(2 \mathrm{v}-6 \mathrm{v})$.
} 
The subjunctive is formed by changing the $i$ of the present tense to qereba; e.g., naqereba or gozanaqereba 'if it be not.'

The permissive subjunctive is formed by changing the $i$ of the present to qeredomo; e.g., gozanaqeredomo 'although he is not.'

The preterit of the subjunctive is formed by adding redomo to the preterit of the indicative; e.g., nacatta redomo 'although he was not.'

The substantive [verb] with the particle tomo is formed with the root; e.g., naqu tomo 'even if it were not.' The gerund is nó, nóte, naqu xite, or nacatte 'since it is not.' The remaining are formed as above, with the verb ari, $u$ added, and are conjugated in the second conjugation.

Adjectives, when they do not precede verbs, are conjugated in the same way as the negative substantive verb. The adjectives, which have been said above to end in $a i, e i, o i$, $u i$, and $i j$, form their roots by changing the final $i$ to $q u$; e.g., fucaqu is the root of 'deep,' ioqu the root of 'good,' xiguequ the root of 'dense,' varuqu the root of 'bad,' and vonajiqu the root of 'the same.'

The present tense is the form (vox) of the adjective itself; e.g., ioi 'good,' fucai 'deep,' varui 'bad,' vonaji 'the same.'

The preterit is formed by changing the $i$ of the adjective to $c$ or $q$ and adding ari,u. This form is then conjugated according to the tense required by the sentence.

The permissive subjunctive with tomo is fucaqu tomo or fucai tomo 'although deep.'

The gerund in Do is fucóte 'since it was deep, ióte 'since it was good,' canaxiúte $\left[\right.$ canaxúte] ${ }^{56}$ 'since it was sad,' xingueote $[\text { xigeôte }]^{57}$ 'since it was dense.' It also takes the form of fucó xite, fucaqu xite, or fucacatte, or again iô xite, ioqu xite, or iocatte.

The adjectives which end in $n a$ are not conjugated. There is, however, a gerund in Do. For example, aqiracana has for its gerund aqiracani xite 'since it was clear,' and with the same meaning there is aqiraca de. Arisóna has arisóni xite 'since it became apparent, or easy to believe.' Ióna has ióni as in ióni xite 'since it is in a good way, or since it has a good manner.' Cava ga fucóte vatarananda 'because the

\footnotetext{
${ }^{55}$ Collado secms to be unaware of the irregularity of vonaji.

${ }^{50}$ Collado is following the general rule established on p. 10 for such forms as caij. $\mathrm{He}$ might better have followed Rodriguez who would transcribe canaxite, as do we.

57 The missing 'closed o' aside, Collado's transcription of this form with an $n$ is indicative of the clarity with which he perceived the nasalization in this context.
} 
river was deep, I did not cross it,' xebobte irarenu 'since it was narrow, he was unable to enter,' varúte cuvarenu 'it is inedible, or it cannot be eaten, because it is bad.' The other tenses of the adjective, as has been said, are formed with the verb ari, $u$ and conjugated according to the requirements of the sentence. The negative conjugation is also formed with ari,u; e.g., if the root is fucacarazu the present tense is fucacaranu 'it is not deep.' 'The preterit is fucacarananda 'it was not, etc.'

\section{The Conditional Particles ${ }^{58}$}

There are five particles which make an utterance (oratio) conditional; naraba, ni voite va, raba, va, and $b a$. When the first two are placed after any verb, affirmative or negative, present, preterit, or future, the result is that the verb becomes conditional. For example; niguru naraba 'if you flee,' iôda ni voite va 'if you had read,' naravó naraba 'if you will learn,' cuvazu ni voite va ${ }^{59}$ 'if you do not eat.' Sometimes voi [voite] is removed from ni voite va; agueô ni va 'if you would offer,' aguetaró ni va 'if you would have offered.' Sometimes voite [voite va] is removed, leaving only ni; e.g., mairó ni coso, nen goro ni mósózure (19) 'if I go, or if I shall have gone, I will tell him so in a friendly way,' xitaró ni coso, saisocu ţ̧uqu maji qere (19) 'if I had done it, it would not have been done with diligence and persuasion.'

The particle raba is placed after the preterit; ${ }^{60}$ e.g., naróta raba 'if I would have learned,' naravananda raba 'if I would not have learned.'

The particle $v a$ is added to the negative roots of all three conjugations; e.g., aguezu va 'if I not offer,' iomazu va 'if I not read,' naravazu $v a$ 'if I not learn,' naqu va 'if it not be,' fucacarazu va 'if it be not deep.'

The particle $b a$ has the same effect and is, like $v a$, joined to the root;

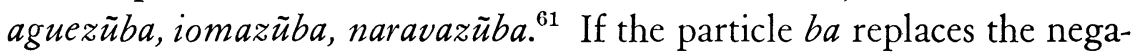
tive $z u$, an affirmative conditional is formed; e.g., agueba, if I offer,' iomaba 'if I read,' naravaba 'if I learn,' and iocaraba 'if it be good.' The particle $v a$ is not only added to the negative roots of adjectives, but also to the affirmative; e.g., fucaqu va 'if it be deep', vonajiqu va 'if it be the same.' Sometimes they use this expression to give the idea 'if it be not

\footnotetext{
${ }^{5 s}$ Cf. Arte $(18 \mathrm{v}-19 \mathrm{v})$.

59 The text reads cil u'aau ni voite va, with the errata changing the verb to cutazu.

${ }^{80}$ This historically inaccurate rule is derived from the Arte $(18 \mathrm{v})$.

${ }^{61}$ In the one example of this construction, on page 62, Collado has the form tovazunba.
} 
too troublesome, will you do it.' They also say aguemajiqu va 'if you would not offer.'

The particle ni voite $v a$ is joined to nouns in such a way as to substitute for the substantive verb; e.g., jójó ni voite va uqe toró $(121 \mathrm{v})^{62}$ 'I shall get it, if it be very good, or the best,' curuxicarazaru gui ni voite $v a$ 'if it would not have been unpleasant, or if it had not been an unpleasant thing.'

If the particle saie is placed in a clause (oratio) in which there is already a conditional particle, it adds strength to the meaning; e.g., fune saie mairu naraba 'if only a ship were to come,' sonata saie vocutabire naku va (118) 'if he be not tired,' or it might be said 'as for me, or as far as it depends upon me, I am not tired.'

The particle saie alone sometimes forms a conditional; e.g., Niffon no xôcocu ni saie caióna coto gozaru fodo ni [Nifon ....] (118) 'if in the small kingdom of Japan things of this kind be found, or exist,' that is to say 'how much more there will be in a large one,' coco moto no tocai ni saie meivacu itasu ióni gozaru fodoni, etc. (118) 'on the voyage here I suffered very much, and so ....' fito saie côquai suru mono vo iurusu ni ivan ia, Deus ni voite voia? [ . . va?] (118v) 'if one forgives one who repents, how much more will God,' core fodo xei vo iruru saie coto naricanuru ni; ucato xite va, incadeca banji canavózo? [ . . icadeca ...] (119) 'if gathering all one's strength this can be done only with difficulty, how could it be done if it were done without any strength?,' core saie xinicui $n i$ 'if this be difficult to do,' fune de saie ioio tçuita ni, cachi va nananaca naru mai [. . nacanaca naru mai] (119v) 'if I arrived by ship with such difficulties, without doubt I could not have done it on foot.'

\section{The Potential Verb ${ }^{63}$}

The placing of the particle $r o^{64}$ after the present or future tense makes a potential; e.g., aguru ró 'he perhaps offers', nigueozuró [nigueôzuró] 'he will perhaps escape.'

The preterit is made by changing $t a$ to $t c ̧ u$ and adding rǒ; e.g.,

\footnotetext{
${ }^{62}$ The original is in the soro style; Iójóni voiteua uquetori mósubequ soro.

${ }^{63}$ Cf. Arte $(19 \mathrm{v})$.

${ }^{64}$ Here and throughout the section Collado transcribes as ro the potential particle which should correctly be written ró (cf. Arte, 11v). It will be noticed that all but one instance of the 'open o' on p. 35 of the text has been left unmarked.
} 
aguetcurŏ 'he perhaps offered.' But if it is added to the negative preterit, the da must be changed to zzu; e.g., aguenanzzuró 'it has perhaps not been offered, etc.'

The present potential is also formed by adding arǒzu [mo arǒzu] or other future verbs to the infinitive; e.g., aguru coto mo arózu or ague mo xôzu 'he will perhaps offer.'

The preterit is formed by adding this same future to the preterit infinitive; e.g., agueta coto mo arǒzu 'he perhaps offered.'

The future is agueô coto mo arǒzu 'he will perhaps offer.' The negative is formed in the same way; e.g., aguenu, aguenanda, or aguru mai coto mo arǒzu 'he perhaps does not offer, he perhaps did not offer, or he will perhaps not offer.' When we wish to say that something is perhaps the case we use mono instead of coto; e.g., noxenanda mono de arózu 'they perhaps did not place it aboard ship,' iqi chigóta mono de arózu 'they seem not to have met along the way,' moreqicoieta mono de gozaró ca to zonzuru 'I believe it is perhaps as it has been said.'

To express the meaning 'become' the verb nari, $u$ is added to the adjective and then conjugated according to the requirements of the adjective taken adverbally; e.g., fucó naru 'it becomes deep,' varü natta 'it became bad.' Also they say fucó aru 'it is deep,' and sometimes fucó nai 'it is not deep.' They obtain this same meaning by conjugating nai according to the tense required by the sentence. They also use fucó nai coto mo arózu 'perhaps it will be that this is not deep.'

\section{The Conjugation of Irregular Verbs ${ }^{65}$}

The verb qi,uru 'to come' has quru 'I come,' qita 'I came,' côzu 'I shall come,' coi or coio 'come!' qitareba 'since he will have come, or would have come,' qitaredomo 'although he came.' The negative root is côzu [cozu] and the negative present is conu 'I do not come.' Mede, which is the root of the verb meaning 'to enjoy,' has a present in mezzuru and its gerund in Do is medete 'by enjoying.' Cui, which is the root of the verb meaning 'to be mournful,' has its present in cuiuru.

\footnotetext{
${ }^{65}$ Collado has derived this list from the Arte $(45-47)$. His terminology is, however, rather misleading. What he classifies as verba irregularia are those which Rodriguez considers deponent, that is verbo defectino, with the term verbo irregular being used by Rodriguez for the adjective. Given this misunderstanding Collado begins his list with an explanation of the irregularities of qi, uru. This verb is on Rodriguez' list only because "it lacks certain forms in the affirmative" ( $45 \mathrm{v})$. Rodriguez has a list of 43 deponent verbs, beginning with tari, from which Collado has selected the first 14 and then a few from the remainder.
} 
Its gerund in Do is cuite 'by mourning,' its negative root is cuizu, and its negative present is cuinu. Araie, which is the root of the verb 'to be, ${ }^{, 66}$ has a present in araiuru or aróru 'it is.' Furi, which is the root of the verb 'to become old,' has a preterit in furita 'he became old,' and a gerund in $D o$ which is furite 'by becoming old.' $F e$, the root of the verb meaning 'to cross over,' has a present in furu 'he crosses over,' and a preterit in feta 'he crossed over.' Tari, $u$ is a verb which signifies that a thing is complete or entire. It has a present in taru 'it is complete,' a preterit in tatta 'it was complete,' and a future in tari maraxo [ maraxô] 'it will be complete.' Its negative root is tarazu, its negative present is taranu, its preterit is tarananda 'it was not complete,' its future is taru mai 'it will not be complete,' and its imperfect subjunctive is taraneba 'since it has not been completed.'

The [negative] permissive is taranedomo, the infinitive is taranu coto, and the gerund in Do is taraide or tarazu xite. The verb taxi:tasu, which means 'to complete, or finish,' has a future in taxi maraxô 'I shall finish.' Tasanu is the negative present. Tari [Tarai] is the root of the verb taró which has the meaning 'to be completed.' In the negative the preterit is taravananda 'it was not completed,' the subjunctive is taravaneba 'since it is not completed,' the permissive is taravanedomo, the infinitive is taravanu coto, and the gerund in Do is taravaide or taravaxu xite [taravazu xite]. Vocotari is the root of the verb vocotaru 'to be negligent.' It has an infinitive in vocotaru coto, a negative root in vocotarazu, and a negative present in voicotaranu [vocotaranu]. Voi is the root of a verb which has a preterit in voita 'he was old.'

Voitaru has the same meaning. The negative present is voinu and the gerund in Do is voite. Urei is the root of the verb 'to be sad.' It has a present in ureô, an imperative in ure io [ureie io $]^{67}$ an infinitive in ureoru coto [ureôru ...]. ${ }^{68}$ Its gerund in Do is ureite. Tomi is the root of the verb tomu or tomeru 'to become rich.' Its preterit is tonda, its gerund in $D o$ is tonde, and its negative root is tomazu. Saiguiri,u means 'to go before, or anticipate.' Its preterit is saiguitta and its gerund in Do is saiguitte.

\footnotetext{
${ }^{86}$ In the restricted context of an adjectival; cf. modern arayuru koto.

${ }^{67}$ Cf. Arte $(45 \mathrm{v})$ where Rodriguez transcribes treyeyo.

${ }^{68}$ Loc. cit. Rodriguez presents vreôrz as an alternative form for vrế in the present tense and then selects that varient for the infinitive.
} 
In this language there are simple active, causative active, passive, neutral, and impersonal verbs. ${ }^{70}$ All are conjugated by the three conjugations according to the way in which their roots terminate.

From certain adjectives come (procedo) certain verbs; e.g., from catai 'hard' comes catame, uru 'I make hard' which is active, catamari,u 'I become hard' which is neutral, catamerare,uru 'I am made hard' which is passive. From the adjective canaxii 'sad' comes canaximi, $u$ which means 'to be sad.'

The causative verbs (verba faciendi facere) are formed with the particles saxe or $x e$. The first is added to the roots of verbs in the first conjugation, ${ }^{71}$ while the second is [not] added to the roots of the second and third conjugation, but rather to the negative present after the $n u$ has been removed; e.g., aguesaxe,uru 'I make him offer', iomaxe,uru 'I make him read,' naravaxe,uru 'I make him learn.' All of these forms are in the first conjugation because the particles end in $e$. Sometimes, but rarely, saxe follows verbs of the second and third conjugation, but this is to make the verbs more elegant. It is used with the particle rare to honor someone; e.g., iomasaxe rare,uru ['he makes him read']. Padre va dojucu ni cathecismo vo naravasaxeraruru 'the priest orders his servant to learn his cathecism, ${ }^{, 2}$ mono no fon vo fito ni iomasaxeraruru (162v.) 'he makes him read his book.'

The passive verbs (verba passiva) are made with the particles rare and $r e$. The particle rare is added to the active verbs, according to the way explained before, after removing the $n u$ from the negative form; e.g., aguerare,uru 'I am offered it,' iomare,uru 'I am read to,' naravare, uru 'I am taught.' They use these passive forms to mean 'to be read to by someone,' or 'to be, or not to be legible.' There are other passive forms which come from neutral verbs or verbs which have neutral meanings. They are also formed with the particles rare and re, but when they are so formed they do not govern the cases common to

\footnotetext{
${ }^{69}$ Formation (formatio) is to be understood here in the sense of derivation, and diversity (differentia) in the sense of class membership.

${ }_{70}$ The opening paragraphs of this section follow the Arte $(68-70$ and $96-108 \mathrm{v})$. The list of particles, beginning with maraxi, follows 160-168.

${ }^{7}$ The text, here and in the next sentence, reads secundae coniugationis.

:2. The form dojucu is incorrect. It is taken by Ötsuka to be doshuk 'a 'a person living in the same house.' The Vocabulario records the item dôjucu 'a young boy who serves a priest.' Dôjucu best fits Collado's translation.
} 
the passive (for which see below) but rather the cases of the verbs from which they come; e.g., from agari,u comes agarare, $u r u$; and, since agari, $u$ 'I ascend' requires the accusative, this verb also requires the accusative. For example; cono iama ie agararenu (102) 'it is not possible to climb this mountain, or this mountain is unable to be climbed,' xiro cara derarenu (102) 'it is not possible to leave the castle,' xebóte irarenu (102) 'it is not possible to penetrate because it is too narrow, or confined,' cono michi va arucarenu (102) 'it is not possible to walk this street,' natçu va coco ni irare mai 'it will not be possible to live here during the summer,' cono fude de va cacarenu (102) 'it cannot be written with this pen,' fima ga nóte cacarenanda (102) 'it cannot be written because of the lack of time,' cono bun ni coso cacaruru mono de gozare $(69 \mathrm{v})$ 'it will indeed be well written in this way,' axi ga itóte arucarenu (102) 'it is impossible to walk because of painful feet.' All of these passive verbs are of the first conjugation. ${ }^{73}$ The neutral verbs (verba neutra) are those which have a neutral meaning; i.e., being initiated by oneself, and not by others. For example; ivo ga toruru 'the fish are caught,' caje ga toruru 'the wind ceases,' ito ga qiruru 'the string is cut,' ji ga iomuru (100) 'the letter [Chinese character] is well read,' aqi,u 'I am uncovered.' Qiri,u 'I cut' is active, qirare, uru is passive, and qire,uru 'I am cut' is neutral. This last form is used when a sword cuts well because it is sharp. Qiraxe,uru is a causative verb which means 'I make someone cut.' Ague,uru means 'I raise,' aguerare,uru 'I am raised' passively, aguesaxe,uru 'I make someone raise,' agari, $u$ 'I am raised' neutrally, agarare,uru 'to be ascendable,' agaraxe,uru 'I cause something to be raised, or I cause him or it to raise himself or itself.' If to these verbs are added the particles which indicate honor (see below) other combinations are made. The adjectives when they are conjugated have a neutral meaning; e.g., fidarui 'I am thirsty,' fucacatta 'it was deep.'

The impersonal verbs (verba impersonalia) do not name or refer to a person; e.g., mi vo fatasu tomo iţ̧uvari vo ivanu mono gia $(69 \mathrm{v})$ 'even if one were to die, one should not tell a lie,' mono mo tabezu saqe mo nomaide ichinichi fataraqu mono ca? (69v) 'is it possible to work all day without eating anything or drinking any wine?', xujin no

\footnotetext{
${ }^{73}$ The text again reads secundae coniugationis.
} 
maie de sono ióna coto vo iu mono ca? 'is it possible to speak this way in front of ones lord?' Concerning the conjugations for these verbs they follow the rules according to their roots.

The root of any verb of whatever conjugation can be taken from its conjugation and changed to another conjugation by adding one of the particles of honor (honor). The resulting form will belong to the conjugation determined by the final letter of the particle. These particles are: maraxi,uru, ari,u, saxerare,uru, xerare,uru, nasare,uru, saxemaxi,u, tamai,ó, rare and $r .^{74}$

The particle maraxi does not add honor to that which is talked about, but rather it is used to speak honorably to those in front of us. For example; cui,u means 'I eat, ${ }^{75}$ but a servant in front of his master will not say nezumi ga cúta 'the mice ate the cheese'; he will rather say nezumi ga cui maraxita. By itself cui, $u$ is in the third conjugation because its root ends in $u i$, but if maraxi is added it becomes a verb in the first conjugation. When we refer to something about a people (natio) we do not show honor to that word but only pay attention to the person we are speaking to by adding maraxi or not. For example, if we are addressing an inferior we say $N a n$ ban jin va core vo cuvanu; but if we are addressing a person of nobility we say Nan ban jin va core vo cui maraxenu 'Europeans do not eat this.' When ari,u is added to the root of any verb it attaches a middling (mediocris) degree of honor; e.g., modori aró ca? 'are you going to come back?' If you add vo in front of the verb it is honored moderately (satis); e.g., vomodori aró ca? 'Your Lordship is going to come back?' Tono sama vo xini atta toqi 'when the master died,' Deus cono xecai vo gosacu atta 'God created the world. ${ }^{, 76}$ We use these particles when we are speaking with honored persons whom we like and with whom we are on friendly terms.

The particle nasare,uru gives the highest (supremus), or moderately great (satis magnus) honor and is placed after the root of the verb; e.g., Deus cono xecai vo gosacu nasareta 'God created the world.'

The particles rare and re add a middling and not a great amount

\footnotetext{
${ }^{7 \pm}$ Cf. Arte (160-164) from which this list and the following material have been derived.

is Throughout his treatment of the respect language Collado glosses his verb forms in the first person, even though that translation might be inappropriate to any context.

${ }^{\pi \theta}$ Rodriguez (Arte, 162v) specifies the distribution of $v o$ and go, using gosacu atta as his example of the construction in context of a Chinese vocabulary item. Collado does not refer to this distinction.
} 
of honor to the verbs to which they are added. The particle rare is added mainly when we are talking about someone who is absent. It is formed by taking the $n u$ from the negative present and replacing it with this particle; e.g., aguerare,uru means 'I offer' when the person to whom the offering is made requires a middling degree of honor and respect (reverentia). This verb coincides letter for letter with the passive but is distinguished from it by the cases which it governs. The particle re is placed after verbs of the second and third conjugation only; e.g., iomare,uru 'to read' and naravare,uru 'to learn,' said of a person having a good reputation. We speak in this way when speaking of those who are equal to us and the servants of our lord, but not of other servants, or nobles.

The particles saxemaxi and xemaxi give the same degree of honor as ari, $u$ and rare or re. These particles are added to the root of a first conjugation verb, ${ }^{77}$ or to the negative present from which the $n u$ has been removed; aguesaxemasu 'he offers.' Maxi,u [Xemaxi,u] is added to the negative present of the second and third conjugation verbs after taking away $n u$; e.g., iomaxemasu 'he reads,' and naravaxemasu 'he learns.'

The particles saxerare,uru and xerare,uru attribute great honor. The first is added to the negative present of verbs in the first conjuga$\operatorname{tion}^{78}$ after the $n u$ is removed, and the second is added to the [other] negatives in the same way; e.g., aguesaxeraruru 'I offer,' iomaxeraruru 'I read,' naravaxeraruru 'I learn.' Because these forms coincide letter for letter with the honorific causative, the particle ari, $u$ may be placed after the verb and the particle vo may be placed before to avoid confusion; e.g., yomaxe aru [vo iomaxe aru] 'I read' and naravaxe aru [vo naravaxe aru] 'I learn.'

The passive verb, concerning which see below, also permits the particle saxerare,uru; e.g., viamavaresaxeraruru (99v) 'I am honored.'

The particle tamai,ó bestows the highest honor. We use it when speaking of God, saints, kings, or generals. It is added to the roots of verbs and conjugated in the third conjugation. It is placed after the root of the passive form when referring to God; e.g., Deus filio, umare

\footnotetext{
${ }^{77}$ The text reads secundae coniugationis.

${ }^{78}$ The text reads secundae coniugationis.
} 
tamó toqi 'when the son of God was born,' Deus agamerare tamó 'God is honored.'

The particle tate matçuri,u makes the meaning of the verb to which it is added humble. It is placed after the root of affirmative verbs; e.g., Deus vo gotaixet ni zonji tate matçuru coto va ichi sugureta jen gia 'to love God is the supreme virtue.' This particle permits some degree of honor if $r e$ is added to it after the final $e$ [i] has been changed to $a$. Thus, when speaking of the saints in respect to God, one says, Sancto Domingo, Deus vo gotaixet ni zonji tatematçurareta 'St. Dominic loved God.'

The particle maraxi $[\text { mairaxi] }]^{79}$ is able to elevate to honor the particle rare; e.g., tono iori cono coto vo Padre ni vataximairaxerareta 'the lord gave it to the priest.'

\section{Certain Verbs Which of Themselves Indicate Honor ${ }^{80}$}

Mesare,uru indicates any act which can be done, or which is properly done by a noble person (persona nobilis). This includes such things as eating, drinking, sailing, riding a horse, etc. Voxxerare,uru means that a noble person speaks. Vomaraxi,uru and vomaraxi ari,u mean that a noble person gives. Voxe,uru [Vôxe,uru] and vôxe ari,u mean that a middling person (persona mediocris) says or declares.

Verbs preceded by vôxe or mexi are given the same degree of honor by either; e.g., vôxe tçuqerare,uru 'I declare, mexi tçucavare,uru 'I serve,' which have the same meanings as ţ̧uqerare,uru and ţ̧ucavare,uru. To call someone we use coi with an inferior, with someone not quite as inferior we use iorai, with someone a little better we use vaxei, while vogiare is the superior way to call. Gozare, which means that your Lordship should come, and gozaró in the future tense are even more honorable ways to indicate the imperative. Voide nasarei, voide nasareô, or voide nasarei caxi mean 'might your Lordship come,' or 'Oh! would that your Lordship come.' Cudasare,uru means that a noble person gives. Tamavari, $u$ means that a noble person gives to an inferior. Tamóri, $u$ means that a middling person gives. Mizzu vo nomaxete tamorre 'Give me a drink of water.' Cudasare,uru and tamóri,u mean

\footnotetext{
${ }^{79}$ Ötsuka (1957) suggests maraxi is correct and alters the example. Since the list begins with maraxi, I assume the error to be in the citation.

${ }^{80}$ The material for this section is derived from the Arte $(164 \mathrm{v}-168)$.
} 
that a humble person eats honoring his food. Coximexi, $u$ and qicoximexi,u mean that a noble person eats and hears. Voboximexi,u and voboximesare,uru mean that a noble person thinks. Saxerare,uru means that a noble person does. Nasare,uru, asobaxi,u, and asobasare,iuru [asobasare,uru] mean that a noble person does what is proper to him such as hunting, writing, reading, or reciting. $I i, \mathfrak{u}$ is used when the person addressed is humbler than the person or thing spoken to; and mexi,u [móxi,u] means the person or the thing spoken to is addressed with honor. Therefore I would be incorrect were I to say $m i n i$ móxe 'tell me!' I should rather say mi ni iie. I should not say tono ni iie 'tell it to the lord,' but rather tono ni móxe. Mairi,u means to go to a place to which honor should be shown; e.g., iglesia ie maire 'go to church!' Cure,uru and toraxe,uru mean to give in a way that humbles the person to whom the thing is given. Cui,ú means 'to eat' without showing respect (respectus); mexi,u also means 'to eat' but it is cultivated (urbanum); e.g., in addressing those deserving respect I will not say mexi vo cui maraxita but rather mexi vo tabe maraxita 'I ate.' Mairi,u or vomairari,u [vomairi ari,u] means that a middling person eats, while agaraxerare,uru and voagari ari,u are nobler ways to say this. Qiqi,u means to hear and uqetamavari,u and uqetamóri, $u$ mean to hear in a way which honors the person heard; e.g., goiqen vo uqetamótta 'I heard your advice.' Móxi ague,uru means to speak in a way which humbles oneself while bestowing honor on the person being addressed. Móxi ire,uru means to speak between equals (equales). Chómon xi,uru means to listen to the word of God. Goranji,zuru or goranjerare,uru is to look at a noble thing. Xi,uru means to do in common way, itaxi, $u$ means to do in a cultivated way, and tçucamatçuri,u means to do in a humble way.

\section{Cautionary Remarks on the Conjugations of the Verb ${ }^{81}$}

The particle nama placed before any verb in any tense means that the action has been done poorly or in an incomplete manner; e.g., nama aró 'I wash poorly,' nama iaqu 'I am incompletely broiled.'

\footnotetext{
${ }^{81}$ While the material for this section is drawn from various sections of the Arte, the bulk of the particles and their descriptions are derived from Rodriguez' treatment of postpositional (73-77) and adverbial constructions (112v-125).
} 
If the particles tçui, cai, uchi, faxe, voi, ai, and tori ${ }^{82}$ are placed in front of a verb they do not change the meaning, but they add emphasis; e.g., uchi cobosu has the same meaning as cobosu 'I pour,' faxe noboru is the same as noboru 'I ascend,' voxi comi, $u$ is the same as comi,u 'I enclose,' ai cavari, $u$ is the same as cavari, $u$ 'I am changed,' tçui mavari, $u$ is the same as mavari,u 'I go around,' and tori firogue,uru is the same as firogue,uru 'I spread out.'

The particle qitte is the gerund in Do for the verb qiri, $u$ and when it is placed after the roots of certain verbs it gives them great emphasis; e.g., tanomiqitte 'imploring with great prayers,' vomoiqitte 'assuming a strong resolution.' The verbs tanomiqiri,u and vomoiqiri,u are also used.

The particle $m a$, when placed in front of certain verbs and nouns, gives them a stronger meaning; e.g., mamucai 'quite present,' macuroi 'completely black.'

The particle, or better root of the verb, macari, $u$, when placed before verbs of motion, makes the verbs modest and a bit more cultivated; e.g., macari noboru 'I ascend,' macari cudari, $u$ 'I descend,' and macari i,iru 'I am present.'

The particle $v a$ placed after a sentence confirms what has been said before, as one might boast of making a prediction; e.g., fune va cuchinotçu ie iru va 'the ship calls at Kuchinotsu; and, if he says so or not, I say so,' aru va 'see if it is not as I have said.'

The particle aidani means 'between' in the sense of the time consumed in performing an action; e.g., agura aidani [aguru aidani] 'while offering,' iôda aidani 'while he read,' naravózuru aidani 'while he will learn.'

The particle ga means 'but;' só iú ga; nanto aró ca? 'they say so, but will it be so?' or 'it may be so, but I don't know for certain,' furi va furu mai ga, fune no dasu coto naró ca xiranu 'it's not raining any more, but I still don't know if it will be possible to launch the boat or not,' sono qinpen ni va gozaru mai ga; doco cara toraxeraruru zo? $(20)^{83}$ 'there are probably none in the neighborhood, or in the surroundings, so from where can they be gotten?'

\footnotetext{
${ }^{82}$ Rodriguez' list $(77 \mathrm{v})$ runs as follows; vchi, voi, faxe, ai, tori, mexi, tçui, and voxi. On the basis of Collado's examples voxi should have been included in his list.

${ }^{83}$ Collado's transcription qinpen is phonemically correct while being phonetically less accurate than Rodriguez' quimpen.
} 
The particle gotoqu is added to the present, preterit, and future tenses meaning 'in the same way'; e.g., coxiraiuru gotoqu 'in the same way as you furnish or carry out,' quita gotoqu 'as I heard.' The form is sometimes ga gotoqu; e.g., móxita ga gotoqu 'as he said,' caracavózu ga gotoqu 'as in jest I will tease or laugh at.' This same meaning is obtained with ionni; Nifon no catagui vo xirareta ióni, vôxeraruru (122v) 'he speaks as one who knows the customs of Japan, ${ }^{, 84}$ mósu ióni 'as I say.' The particle furi is also used for the same purpose; e.g., toza no chijocu vo nogareôzuru tameni catana vo saita furi vo mixerareta (123) 'he showed himself wearing his sword in order to avoid the danger of infamy.' minu furi vo saxerareta (123) 'he made it known that he did not see.'

The particle saie is used [with the negative] to mean 'not at all'; e.g., mma saie nacatta (118) ${ }^{85}$ 'there are not any horses at all,' cotoba saie xiranu mono (118) 'he does not know how to speak at all,' ji saie mixiranu mono 'he does not know any letters at all.' This same particle is used for emphasis; e.g., qiden to saie móxeba (119) 'it would (44 suffice if you were to say that you are,' Padre no tçucavaruru to saie móxeba 'if only he had said that this was useful to the priest,' or one might say 'it would suffice if, etc.'

The particle qere is a confirmative particle which comes at the end of a sentence with the meaning 'therefore'; e.g., maitta qere 'therefore he came,' sate só aru qere 'finally this is the situation. ${ }^{, 86}$

The particle coso is of great importance among the Japanese for they use it first in an adversative sense (in sensu adversativo); core coso ió gozare $[\ldots i \hat{o} \ldots]$ ' he is truly good. ${ }^{187}$ If the sentence in which this particle is found ends in a verb, that verb ends in $e$, as in the example above. If the verb is in the preterit it ends in re; e.g., yô coso gazattare! (117) 'you are welcome! (bene veneris!).' The exceptions to this rule are when the sentence does not end in a verb or an adjective; e.g., core coso xixó yô [...io] (116) 'he is a true teacher,' when after the particle coso there is in the sentence a gerund in $e$, a permissive in tomo, or a

\footnotetext{
${ }^{84}$ Collado has altered Rodriguez' version from Nippon, even though the Dictionarium glosses consuetudo japonica as Nippon catagui.

${ }^{85}$ Collado, in the Dictionarium and here, prefers $m m u$ to $u m a$.

${ }^{88}$ This particle is not described in the Arte.

${ }^{87}$ Rodriguez (Arte, 116) records Core coso yocarózure and states that in this context coso has the same meaning as Queccu and Cayette.
} 
potential preterit in ţ̧uró or zzuró $;^{88}$ e.g., vare coso iro iro xinro tçucamaţutte cutatireba toxiiórini nari maraxita [. . cutabireba toxiiorini...] (117) 'suffering many and various hardships, I became an old man,' vare coso corosaruru tomo 'if I be killed,' fara coso tatţuró (117) 'he was perchance quite angry,' sato chicaqereba coso fi ga miiure (116) 'the fire is already seen because the village is so near.' This [last] sentence ends in $e$ because it does not contain an exception to the rule. Vôxerareta coto domo vo go côquai de coso gozarózure (97) 'without doubt you will do penance for what you have said,' catajiqe nó coso gozare (97) 'I congratulate you very much and thank you.' If someone says, 'Who did that?' the answer is Patre coso [Padre coso] 'the Priest did.' If someone asks, 'is there anyone who did that?' and if he does not hear, or does not understand the answer, and asks again, the person who answered will say Juan coso 'I have already said it was John.'

When someone is careless about what was said, or when he has not heard something and asks again, the answer is; e.g., tovoru na to iieba 'I have already told you not to pass through,' iome to iieba 'I have already told you to read,' Padre coso to iieba 'I have already told you that it is the Priest.'

Adding the particles maieni and saqini to the negative present tense makes the construction affirmative; e.g., iglesia ie mairanu maieni (141v) 'before he goes to church.' They are also added to the affirmative future tense; e.g., mairözuru tote no saqini 'a little before I come.'

The particle tocoro signifies the time during which the action indicated by the verb is done. It is placed after the verb; taburu tocoro $n i$ 'when I was eating,' tabeta tocoro ni 'after dinner,' tabeôzuru tocoro ni or tabeôzuru $n i$ 'when I will be eating.' It also serves as a reduplicative particle which denotes a reduplication to the degree possible; e.g., jesu christo humanidad no von tocoro va $(121 \mathrm{v})^{89}$ 'Jesus Christ in so far as he was a man,' vonore ga foxxezaru tocoro vo fodocosu coto nacare (121) 'as you do not want done to you, do not do to others,' fudai no tocoro vo vo iurusu [. . . tocoro vo iurusu] (120v) 'I gave him his freedom,' fito no acu no tocoro ni va dôxin xenu (121v) 'I do

\footnotetext{
${ }^{88} \mathrm{Cf}$. the Arte (117) where the list is given as Reba, Ni, Tomo, the potential, and Te.

${ }^{89}$ Rodriguez' version runs lesu Christo fitono vontocoroua. (For Collado's use of reduplicatiuus see note 12.)
} 
not consent to the sins of man,' utagó tocoro mo nai (120v) 'there remains no place to doubt, or for doubt,' nocoru tocoro mo nai 'it does not remain any more,' ţ̧uini, sono tocoro ie mairózu (121v) 'finally he will arrive at this place,' fumbet ni voiobanu tocoro gia (121v) 'there are some things which are not understood, or to which one's comprehension does not extend,' nani mo nai tocoro vo iô qicoximexe (120v) 'will your Lordship kindly eat from this littleness which is nothing.' From these examples it is possible to see the force of this particle.

The particles tocoro, made, and made de gozaru are often added to an utterance (cadentia). They do not have any special meaning and are the same as coto de gozaru; e.g., naranu made or naranu coto de gozaru mean the same as naranu 'it is not possible.' Guijet tçucamatçuró to zonzuru coto va cacugo itasanu coto gia (10v) 'the breaking of this friendship does not come to mind.' Here the itasanu coto gia is the same as itasanu alone.

The particle madeio is used to confirm what has been said; e.g., caita madeio 'that which I wrote, I wrote.'

The particle toqi when added to the present tense, forms a preterit imperfect; e.g., jennin tachi va saigo ni voiobi tamó toqi va buji ni gozatta 'when saints arrive at the time of their death, they are peaceful and quiet.'

Changing the $t a$ of the preterit to $t c ̧ u$ and the $d a$ of the negative to $z z u^{90}$ the meaning becomes ' $I$ do it this way and then that way'; e.g., mono vo caitçu, iôzzu, nando xite curasu bacari gia 'I spend my life reading, writing and doing other things,' tatţ̧ itçu vocu iori zaxiqi ie ide zaxiqi iori vocu ie iri xitten batto xeraruru (11v) 'standing and sitting, entering and departing, he stands up and falls down.' The particle $r i$ gives the same meaning after the preterit; e.g., xeqen no mono va netari voqitari nódari curasu bacari gia (11) 'men of the world spend their lives sleeping, arising, and drinking,' mazzu ite niva vo mo facaxetari, cusa vo mo ficaxetari iroiro no xigoto vo ategóte cosó mairǒzure [ . . coso ...] (10v) 'I shall go and sweep out the courtyard (atrium), pull up the weeds, and then having dispensed with these things I shall go,' ima cono io fuqe iuqeba nome ia, utaie ia fito bito motçu, utótçu sacamori suru (129) 'when it already is late at

\footnotetext{
${ }^{90}$ As the first example indicates, the $z z u$ varient is not restricted to the negative preterit, but is the form which appears for $d a$ in all contexts, as here with the preterit of iomu.
} 
night, urging themselves on to drink and sing, the men enjoy themselves dancing and singing.'

The particle ie, which is the root of the verb ie iuru [ie:uru ${ }^{91}$ 'I can,' signifies, when placed before negative verbs, that the action expressed by the verb cannot be done; e.g., ie iomanu 'I cannot read.' This particle is also placed after the infinitive; e.g., iomanu coto vo ienu 'I cannot read.' Iomi va ieide, or iomi mo ieide 'since I could not read, or not being able to read' is also said. The infinitive sometimes acts as a substitute verb (suppositum verbum); e.g., xinuru coto va vosoroxij 'it is terrible to die.'

The particle tai 'I want' is added to the roots of verbs and signifies the desire to do the thing indicated by the verb; e.g., mizzu vo nomi $t a i^{92}$ 'I want to drink some water', mizzu vo nomi tó gozaru or mizzu vo nomi tó zonzuru, but these last two forms are more noble. Here is an example of the noble form in the negative, to mo nai; e.g., mizzu vo nomi tó mo nai 'I do not want to drink water,' and mizzu vo nomi tó mo gozaranu. Mairu tó mo zonjenu means 'I do not want to go.' When the particle tai is added to adjectives, or verbs indicating a sensory act (actionem sensitiuam) in the first person, ${ }^{93}$ the $i$ is changed to $c$; and the verb ari, $u$ is added and conjugated in the tense required by the sentence; e.g., cuitacatta 'I wanted to eat.' If the verb is in the second or third person, the $i$ is changed to $g$ and again the verb ari, $u$ is added, or an honorific particle depending upon what the person deserves, or without it as an absolute form. But if the person is inferior, the $i$ is changed to $c$ as said before.

The particle de sometimes gives a subjunctive sense when it is added to nouns; e.g., varãbe de xinda 'he died a child, or when he was a child,' vare ga buchófó de tofó mo gozanai (163v) ${ }^{94}[\ldots$ buchôfó . . . ] 'since I am clumsy and not careful, nothing will work out in a way that will be harmonious.'

The particle ió 'way' is added to the roots of verbs and also to the

\footnotetext{
${ }^{91}$ In the absence of other examples it is not possible to determine if Collado assumed the present tense form to be iuru or uru. The correction here follows the spelling used consistently in the Arte.

${ }^{92}$ Both Collado and Rodriguez agree that verbs ending in tai govern the accusative case; cf. Nanigaxiuo yobitai (Arte, $14 \mathrm{v}$ ).

${ }^{93}$ The text reads secunda persona.

${ }^{94}$ Rodriguez has Vatacuxiua nantomo buchôfôde tofó ga gozanai [ . . buchôfóde . . . ].
} 
verbs themselves. When the root governs the genitive, the verb governs the same case; e.g., cono qió no iomi ió va'the way of (47 reading this book,' or cono qió vo iomu ió va. In the first sentence qió is in the genitive with the particle no; in the second sentence it is in the accusative with vo because yomu governs this case. Tei signifies an extraordinary and marvelous way of doing something; e.g., machicanuru tei vo goron jerarei (122) ${ }^{95}$ 'might your Lordship observe the way that they are expectant.' Also, arisama means 'way', me mo aterarenu arisama gia 'it is a way, or a form (figura), which is unable to be seen.'

Sama indicates the time of the action of the verb to which it is added while governing the case required by the verb. It is added to the root of the verb; e.g., saqe vo nomi sama ni (105) 'when he actually drank the wine,' iado ie caieri sama ni (105) 'when he returned home,' fune iori agari sama $n i$ (105) 'when he actually disembarked from the ship,' fune ni nori sama $n i$ ' when he actually boarded the ship.'

When there are in a sentence two verbs whose actions form a single action, the first verb is put into the form of the gerund in $D_{o}$; e.g., mizzu vo motte coi 'bring some water, or come bringing water,' fune vo voite coi 'bring the boat here, or come poling the boat,' core vo totte iqe 'take this, or carry this and go.'

The gerund in Do when added to verbs of asking, giving, or doing, means that one is asking to know or to acquire the thing which is indicated by the verb to which it is added; e.g., nifon guchi vo voxiiete cure io 'teach me Japanese,' só voxerarete cudasaruru na [só vôxerarete ... ] 'your Lordship ought not say that,' Deus no coto vo catatte tamóre 'do me the favor of relating to me those things which pertain to God.'

The particle mo placed after the gerund in $D o$, whether it ends in te or de, means 'although'; e.g., só móxite mo 'although you say so,' ica fodo susumete mo, corobu mai 'no matter how much you try to persuade me, I will not deny the faith.' They also use só móxeba atte mo 'even if you say that,' dóxitemo cóxitemo (134v) 'what ever you do.'

If the particle coso (see above) is added to the affirmative gerund in Do; and, if the sentence ends in this particle, the sentence becomes nega-

\footnotetext{
${ }^{95}$ Rodriguez uses the transcription gorójerarei in the example from which this sentence is derived. (The ten other occurrences in the Arte have goran.) The Dictionarium uses only goron, while the Vocabulario lists both goran and goron. The Spanish manuscript has goran.
} 
tive; e.g., mite coso 'I did not see anything,' atte coso 'there is no way.' But if the sentence does not end in coso, it becomes affirmative and emphatic; e.g., mite coso gozare (116) 'I certainly saw.' The verb ends in $e$ according to the rule explained above when the particle coso was being discussed.

When the negative gerund in Do, which ends in $e$, is followed by $v a$, naranu, or canavanu it expresses necessity or the impossibility of the contrary; e.g., mairaide canavanu (106v) ${ }^{96}$ 'it is necessary to go,' ivaide va no coto naredomo, nanto xô ca? 'and if the thing which is said to be necessary happens, what shall I do?' xitagavaide naranu 'it is necessary to obey.' The same meaning, but with less strength, is obtained with the future of the affirmative or negative infinitive and the permissive subjunctive in domo; e.g., mairó coto de gozatta redomo $(18)^{97}$ 'although I should have gone,' mairu mai queredomo [. . . qeredomo] (18) ${ }^{98}$ 'although I should not be going,' mairó coto de gozanacatta redomo (18) 'although I did not have to go.' They also use the negative gerund in $D o$ to obtain the meaning of 'if not'; e.g., òracio vo mósaide cú na 'do not eat unless you have said your prayers.'

The gerund in $e$ indicates an action already done; e.g., mexi cuite coi 'come after eating!' cono qió ga caite gozaru 'this book was written,' chichi ni fumi vo cacaide cuiaxiú gozaru [...cuiaxú...] 'I am ashamed that I did not send a letter to your father,' cono qió ga caite gozaranu 'this book was not written.'

The particle nagara, when added to the root of a verb, forms a gerund in $D o$ if it is followed by a verb indicating a repugnant or contrary action; e.g., toganin Deus iori bacutai no go von, o uqetatematçuri nagara; caietta somuqi tatematçuru $[. . \text { go von vo uqe ... }]^{99}$ 'sinners receiving, or even if sinners receive, benefits from God, they will offend him rather than be grateful,' Jesu Cristo Deus de gozari nagara, fito ni taixite cruz ni cacaraxerareta 'while Jesus Christ was a God, he was crucified for man.' Nagara is also added to nouns; e.g., quantai nagara (136v) 'although there was some lack of education,' sannin nagara (137) 'three at the same time, or even if there are three' aqiraca

\footnotetext{
${ }^{90}$ Rodriguez has mairade canatuanu.

97 The Arte has the plain form mairócotode attaredomo.

${ }^{98}$ The Arte has mairumajiqueredomo.

${ }^{90}$ Perhaps an attempt to follow the rule, established in the syntax below, that states the $v$ of the accusative particle is lost after $n$. If this is the intent, the comma is in error.
} 
nagara (136v) 'although he is famous.' In this instance aqiraca na loses its $n a$ as do all the other adjectives that end in $n a$.

The particle iasui is added to the roots of active and passive verbs to form the supine in Tu; e.g., iomi iasui 'easy to read,' corosare iasui 'easy to be killed.' The same thing is achieved by the following way of speaking; iute va vosoroxij 'it is terrible to say' mite va fuxiguina 'it is admirable to see' iú vo mo vosoroxij 'it is terrible to say.'

\section{The Adverbs}

\section{First Section ${ }^{100}$}

Adverbs are formed from adjectives ending in $a i$ by changing the ai to ó; e.g., fucó 'deeply,' for those ending in oi by changing the oi to $\hat{o}$; e.g., caxico [caxicô] 'wisely,' for those ending in ei by changing the ei to ế ; e.g., xigueo [xigueô] 'densely,' for those ending in $u i$ by changing the $u i$ to $u$; e.g., aiu 'in danger,' and for those ending in $i j$ by changing the ij to iú; e.g., cavaiú 'unhappily.'

\section{Adverbs of Place $e^{101}$}

The interrogative pronouns are eight in number; izzucu[?], izzucata[?], donata[?], doco?, dochi?, dochira?, dono tocoro[?], and dono fó?, and they signify 'which place?' To these adverbs are added the particles $v a, n o, n i, i e, v o$, cara, and iori according to the case required, such as 'from where,' 'whither,' 'through which place,' 'in what place,' etc. Made can also be added to them with the meaning of 'to the limit of which'; doco made ie iqó ca[?] 'up to where will you go?' The interrogative particle, ca? or $z o[?]$, is added to these questions but it is better to use $z o$ rather than $c a$ in sentences with an interrogative particle; e.g., izzuru ie maitta zo 'where did you go,' dono tocoro vo tovotta zo 'at which place did you cross,' doco iori itta zo 'through where did he enter,' dochi cara qita zo? 'from where did he come?',

\footnotetext{
${ }^{100}$ Rodriguez treats adverbs in two sections of the Arte; under the parts of speech $(73 \mathrm{v}-77)$ and under the syntax (113-125). As has been observed in the introduction, there is little consistancy of classification between Rodriguez and Collado in this area of grammatical description.

${ }^{101}$ The interrogatives are derived from the Arte $(110 \mathrm{v})$ and are presented in substantially the same order. The adverbial particles which begin with aie are taken from $(140-148 v)$ and classified by Rodriguez as posposiçao.
} 
donata va Pedro no iado zo[?] 'which is Peter's house?', doco ni voru $z o[?]$ 'where, or in what place is he?' One may respond in many ways; cono tocoro, coto moto [coco moto], core, conata, cochi, cochira, coco, cocora, cono cata, cono fó, which mean 'here (hic)'; sono tocoro, soco moto, sore, sonata, sochi, sochira, soco, socora, sono cata, sono fó $\quad(50$ which mean 'there (istic)'; ano tocoro, asoco moto, are, anata, achi, achira, asoco, asocora, ano cata, anofó, which mean 'there (illic).' To these particles are added the case particles. The interrogative adverbs with the case particles and mo added mean 'everywhere,' 'through every place,' or 'to every place,' e.g., dono tocoro ie mo tovoró 'I shall go everywhere,' doco ni mo 'everywhere,' doco cara mo 'from everywhere.' However, if, instead of mo, nari tomo is added the meaning becomes 'any place,' in a distributive sense; e.g., doco ie nari tomo mairó 'I shall go to each place individually.' The same meaning is obtained by doco zo with the case particles placed between the doco and the zo; e.g., doco $n i$ $z o$ aru fodo $n i$ 'if someone is any place.' Coco caxico means 'here and there.' Doco mo caxico mo means 'the whole place.' The case particles are placed before mo; e.g., doco ni mo caxico ni mo 'in the whole place,' but after the adverb; e.g., coco caxico ni 'here and there,' coco caxico ie doco, caxico iori [ coco caxico ie 'to here and there,' coco caxico iori 'from here and there'], etc. ${ }^{102}$

The particle wie means 'above.' The genitive case is placed before it; e.g., fandai no wie ni voqe 'place it on the table,' cono mie va gozaru mai 'it will not be above this,' that is to say 'it will not be better than this,' sono wie ni 'about that,' sono wie no sata vo catari are 'tell me about that,' core va izzure iori mo uie de gozaru 'one will not discover anything better than his,' that is to say 'this is the best.' Xita means 'below.' It governs the genitive; e.g., fandai no xita ni voqe 'place it under the table,' micotoba no xita iori (141v) 'when the king finishes speaking,' voxita vo cudasarei (141v) 'would your Lordship be so kind as to give to me that which remains of your drink.'

The particle soba means 'side' and governs the genitive; e.g., fito no soba vo fanaruru 'he separates himself from the side of another.'

The particle maie means 'before' and governs the genitive; e.g., fito no maie vo tovoru 'I pass in front of someone else,' cacugo no maie

\footnotetext{
${ }^{102}$ The errata has; page 50, line 10, doco read coco. This would require the doco zo above to read coco $z o$. It seems that the crrata should have read; page 50 , line 16, which would have corrected this error. The punctuation is not corrected by the errata.
} 
(141v) 'according to ones disposition,' funbet no maie (141v) 'as I believe, or think, or according to the sense (iuxta sensum).'

The particle mavari means 'around' and governs the genitive; e.g., iglesia no mavari ni tçuchi vo nague sutçuru na' do not put earth around the church.'

The particle uchi means 'within,' and the noun which precedes it must be in the genitive; e.g., iglesia no uchi 'in the church,' ano fito va, fito no uchi de va nai 'that man is not among men,' that is to say 'he is not a man,' futacuchi cúta coto va, cúta uchi de va nai $(142 \mathrm{v})^{103}$ 'to eat two mouthfuls is not to eat.'

The particle foca means 'outside,' and the genitive is placed before it; e.g., igelsia no foca ni 'outside the church,' foca ie iqe 'go out, or go outside.' Sometimes the genitive particle is replaced by iori; e.g., Deus vonago ichinin iori foca tçucuri tamavanu $(142 \mathrm{v})^{10 \pm}$ ' God did not create but one woman,' that is to say 'he created just one,' Tengu fito ni acu vo susumuru iori foca va, nai (142v) 'the Devil does nothing if he is not persuaded by man to sin,' goxó vo tasucaru tame baptismo vo sazzucaru iori foca bechi no michi ga nai 'there is no other way to save men than by baptism,' that is to say 'without baptism we cannot be saved.' Deus no gracia iori foca 'without the grace of God.'

The particle naca means 'in the middle.' It is used when the material is either dense or defuse; e.g., qi no naca ni 'in the wood,' fito no naca ni 'among the men.'

The particle nacaba means 'in the midst of things' when referring to a sequence. It follows the genitive; e.g., dangui no nacaba ni 'in the midst of the sermon,' sore vo qijte, nacaba va vosore; nacaba va aqirete ita $(145 \mathrm{v})$ 'hearing that, he feared and was afraid,' that is to say 'he spent most of his time being afraid.'

The particle ato means 'behind' and governs the genitive; e.g., sonata no ato cara mairó 'I shall come after you' that is to say 'I shall follow you.'

The particle vaqi means 'near' and governs the genitive; e.g., Pedro no vaqi 'near Peter,' misa no vaqi 'the mass is ended,' cono vaqi in the last few days.' All of these adverbs require after them the cases that are required by the verb which follows.

\footnotetext{
${ }^{103}$ Rodriguez has the complete version; Fitocuchi futacuchi cù cotona crita vchideua nai.

${ }^{104}$ Rodriguez uses vonna for vonago.
} 


\section{Adverbs of Interrogation and Response $e^{105}$}

There are many ways to ask 'why?' or 'for what reason[?]'; e.g., najeni[?], najoni[?], nani xini?, nani tote ca?, nani no iuie ni?, nanto xita coto ni?, nani no xisai ni iotte?. The question 'how?' is said; nanto xite?, nanto io ni[?], icani to xite? The answer is 'because' or 'for the reason that'; e.g., sono iuie va, najeni to iuni. 'Because' is also said; tocoro de, fodo ni, ni iotte, or sacai ni. The first expresses the greatest degree of causality, the second not so much, and the third the least.

Uie va means 'since (cum or si quidem)'; e.g., toganai uie va qizzucai ga nai $(40 \mathrm{v})^{106}$ 'I am not afraid because I have no fault.' The same meaning is achieved by the particle cara; e.g., caio ni iro vo misuru cara va; cacusu coto va iranu' since you have thus shown your feelings (iro), you can't hide them.' 'Since (si quidem)' means approximately the same as toqi va and xicaru toqi va. Sari nagara means 'but,' sari tote va means 'until,' saru tote va means 'since the thing is this way,' saru tote va, qicoienu coto gia 'since it is so, it is unbearable.'

\section{Adverbs of Time Tin $^{107}$}

One asks 'when' with iţ̧u or iţ̧ugoro. One asks 'from what day' with icca saqi or icca maie, 'from what month' with icutcuqi saqi, and 'from what year' with nannen maie. Usually $n i$ is added when it is required by the verb, and the interrogatives $c a$ or $z o$ are always put at the end of the sentence, with $z o$ preferred.

One answers 'now' with ima or tada ima, and 'already' with mó, e.g., mó iqe 'be already gone!' 'Sometimes' is said with toqi ni iotte or jibun ni iotte. 'Afterwards' is nochi. Sore cara or sore iori means 'after that,' core cara or core iori means 'after this,' and are iori or are cara means 'after that.' 'Immediately' is said with iagate. 'Afterwards' or 'again' is ima iori nochi, ima iori xite va, or ima iori igo. 'This morning' is said with qesa. Connichi or qio [qiô] is 'today,' and asu or miônichi [miónichi] ${ }^{108}$ is 'tomorrow.' 'Tomorrow morning' is asa, axitatô, or

\footnotetext{
${ }^{105}$ The material for this section is derived from the Arte (74v and 76v).

${ }^{100}$ Rodriguez has Ayamari nai vyeua , . . $107 \mathrm{v}$.

${ }^{107}$ For the temporal interrogatives cf. Arte $(89 \mathrm{v}-90 \mathrm{v})$ and for the remaining forms 107 -

${ }^{108} \mathrm{Cf}$. the Dictionarium under cras.
} 
asatocu, and 'tomorrow night' is mionia [mióia]. 'Before' is ijen or saqi ni. 'Yesterday' is qinô or sacujit. 'The day before yesterday' is vototoi or futçuca saqi ni. 'Several days in the past' is cono giv. Cono fodo and xenjit have the same meaning, as does xendo. Condo means 'several days in the future.' 'The day after tomorrow' is asatte or miógonichi. 'Three days hence' is xiasatte or miómiógonichi. Qiônen [Qionen] or cozo means 'last year.' 'This year' is cotoxi. 'Two years ago' is vototoxi or votodoxi. 'Three years ago' is sanuruvotodoxi [sannuru votodoxi]. ${ }^{109}$ 'Immediately' is tachimaqi [tachimachi] or socuij ni [socuji ni]. Sunavachi is also 'immediately.' Tanteqi is 'in a moment.'

Itçumade? means 'until when?' Itçumademo means 'always.' Itçu cara means 'after what time.' Itçu iori means 'from what time.'

\section{Adverbs of Negation ${ }^{110}$}

Iia or iia [ïa ïa] ${ }^{111}$ means 'not.' Só devanai means 'it is not so.' Iccana or iccanagueni means 'by no means,' iume iume means 'not even in a dream,' sarani, ichiien, catçute, or catçute motte means 'in no way,' and io, iomo, or iomo iomo means 'without thinking'; e.g., catçute mairu mai 'in no way shall I come,' iomo só va gozaru mai $(117 \mathrm{v})$ 'it will in no way come to mind why it will be so.' When affirmative verbs are added to these adverbs they become negative; e.g., iomo iomo to móxitareba vo mairi atta $(117 \mathrm{v})$ 'although you said you would not go, you went,' io mairó 'in no way shall I go.'

\section{Adverbs of Affirmation ${ }^{112}$}

Nacanaca means 'it is so,' vó means 'so,' when one agrees. Gueni or gueni gueni means 'it is thus'; e.g., gueni gueni só mo aró 'without doubt the situation is thus.' Chódo means 'at all.' Saionni, sono bun, sono gotoqu, só de gozaru, sore sore, massó gia, or xicato means 'it is so.' Mottomo means that something is reasonably said. Guioi no gotoqu means 'as your Lordship believes, or says.' Mochiron indicates that a thing does not come in to doubt or discussion. Nacanaca naru

\footnotetext{
${ }^{109}$ The Vocabulario has sanumu and sannuru as the ombin form of the attributive perfective sarinuru.

${ }^{110}$ Cf. Arte $(74 \mathrm{v})$.

${ }^{111}$ The Spanish manuscript has iya iya.

${ }^{112}$ Cf. Arte $(74 \mathrm{v})$.
} 
coto de gozaranu means 'truly it is not possible.' Nacanaca no coto indicates a thing with which it is possible to agree. Macotoni means 'truly,' as does xinjit or xinjitni. Xeimon means 'I affirm by oath.' Isasaca or isasaca motte means 'not even a little,' and issai or ixxet means 'in no way, or by no means,' and when these particles are added to the affirmative they mean 'truly.'

\section{Comparative Adverbs ${ }^{113}$}

Iori, iori mo, and iori mo navo mean 'more' in a comparison. The person compared is in the nominative case and the person to whom he is compared is in the ablative with one of the particles which we have listed above; e.g., Pedro va juan iori mo gacuxó de gozaru 'Peter is wiser than John,' soco ie noboru iori va; mairanu ga maxi gia 'it is better not to go than to climb up there.' Gotoqu, mama, and ioni are adverbs of similitude (adverbia similitudinis) and require the genitive for the thing with which the comparison is made. If the particle is preceded by a verb, no genitive is required; e.g., no iama ie nari tomo qitai mama ni qite, nurureba, nugui suteraruru (124v) 'if they were to go to the mountains or the plains wearing such clothes as they want to wear, they will have to take them off when they become wet on account of the water.' Vomô mama ni, vomô gotoqu, and vomô ióni, mean 'as I think,' cono mi no mama ni 'according to his desires, or his pleasure.' Fodo means 'to such a degree as (tantum), or 'just as (quasi)'; e.g., qifen ano fito fodo no gacuxó de gozaru' ${ }^{114}$ 'you are as wise as he,' fara ga cudaru fodo ioi 'he will recover as soon as he has a bowel movement,' michi vo aruqu fodo cutabiruru (123v) 'as I walk so I get tired,' acai fodo ioi 'the redder the better,' xinuru fodo no vazzurai de va nai 'this disease is not strong enough to cause death,' fune ni mesaruru fodo naraba vare mo norózu (124) 'if Your Lordship would take up the task of boarding the ship, so shall I,' tamexi mo nai fodo ni atta to mósu (124v) 'they say it was as if it had never been,' voquru fodo araba sore ie mairozzu (124) 'if I am able to arrive at the state where I can get up from bed, I shall come to you,' chicara no fodo vo mite 'seeing the degree of his strength,' fodo nó tçuita 'he arrived in

\footnotetext{
${ }^{113}$ Cf. Arte $(75,94 \mathrm{v}$, and $123 \mathrm{v}-124 \mathrm{v})$.

${ }^{114}$ Cf. Arte (94v) Quixoua ano fito fodono gacuxódeua nai.
} 
an instant,' core fodo 'as this,' sore fodo 'as that,' are fodo 'as that,' vovoi fodo 'while more,' sucunai fodo 'while less.'

\section{Superlative Adverbs ${ }^{115}$}

Uie means 'the highest'; e.g., christian no voxiie va izzure iori mo wie de gozaru 'the doctrine and faith of Christianity are supreme, or above all,' cono saqe no wie va nai 'there is no better wine than that.' Ichi or daiichi means 'supreme, or unique'; e.g., gacuxó no uchi ni Sancto Thomas daiichi de gozatta 'among wise men Saint Thomas was the best,' core va are iori mie 'this is superior to that.' The particle xita has the opposite meaning of 'inferior, or the lowest'; e.g., xiqitai va anima iori xita de gozaru (141) 'the body is inferior to the soul.'

\section{Adverbs of Intensity and Exaggeration ${ }^{116}$}

Ichidan, chicagoro, and iccó mean 'intensely (valde)'; e.g., chicagoro no vo cocoro gaqe de gozaru 'this is the greatest care and diligence,' sore va icco varui coto gia 'this is extremely bad.' Bexxite means 'chiefly,' tori vaqe means 'especially,' coto no foca means 'rarely, or extraordinarily,' icanimo means 'intensely,' and amarini means 'too much.' As has been said, adverbs are formed from adjectives according to the rules above, and these adverbs mean adverbially what the adjectives mean adjectivally; e.g., fucai means 'deep,' and fucó means 'deeply.' Icani mo xizzucani means 'extremely quietly,' tani coto ni means 'extraordinarily,' and xitatacani or guiósanni means 'in a way that is to be feared' that is to say 'too much.' See the dictionary. ${ }^{117}$

\section{Accumulative Adverbs ${ }^{118}$}

Voxinabete means 'universally'; sôbet means 'generally' as do ţ̧uneni and sojite [sôjite]; feijeini means 'regularly'; and voioso, tabun, vocata, ioppodoni mean 'for the most part,' and qeccu or caiette means 'after all.' Tennen means 'perhaps,' as do xijen and icasama. Sadamete means 'probably,' canarazu means 'without doubt,' moxi xijien [moxi xijen] means 'perhaps,' xótocu means 'naturally,' jinen

\footnotetext{
${ }^{115} \mathrm{Cf}$. Arte (95 and 141).

${ }^{116} \mathrm{Cf}$. Arte (75).

${ }^{117}$ The Dictionarium has a selection of a dozen intensifying adverbs listed under valde.

${ }^{118}$ Cf. Arte $(74 \mathrm{v}, 75$, and 76$)$.
} 
means 'by chance,' xidai vidai ni or jen jen ni means 'gradually,' and vonozzucara means 'by oneself.'

\section{Adverbs that Conclude and Claim Attention ${ }^{119}$}

Ficqiǒ and tçuini mean 'finally, or in conclusion.' Ţ̧ugó means 'in summary.' Nó nó means 'is it not so?' e.g., nó nó icani qicaxeruru ca? 'do you hear me then?' Moxi ${ }^{120}$ means 'ho there (heus),' but it is an elegant word; e.g., moxi Padre sama 'ho there, Reverend Father.' Iare also means 'ho there,' but with inferiors; e.g., iare taró quaja to iieba 'saying "Ho there, Tarōkaja."' Iai means 'ho there' with very low people; e.g., iai sochi ga motta mono va nani zo? 'hey! what is it that you bring?' Ia has the same meaning; e.g., ia vo tono bara domo va nani vo savagu zo? (128) 'hey! you soldiers and good men, why do you quarrel?' The particle ai has the same meaning but it is placed after the sentence; e.g., izzure mo mina qiqe ai (129) 'hey! all of you listen.'

The particles $c a$ and $z o$, as has been said above, are used as interrogatives. The particles $i a$ and caia have the same function but they are more humble; e.g., are va tare caia? 'who is he?', core ia[?] 'this?', io fuqete tare ca va tazzuneô $z o$ ? (89v) 'when it becomes late at night, who will be able to visit?", sore de aró ca to iú coto gia 'I said, "will it be this?"'

No? asks for agreement; e.g., gozaró ca no? 'will he come?'121 mairó to voxerareta no? [. . vôxerareta no?] 'did he say that he will come?' no Pedro dono? 'isn't that so, Peter?' $\mathrm{Na}$ [?] means the same thing, but it is used with inferiors; e.g., só quita na? 'didn't you hear so?' Sometimes, in a sentence containing zo, baxi, which is a dubitive particle (particula dubitandi), is placed; e.g., nanto xita xisai de baxi gozaru zo? $(122 \mathrm{v})^{122}$ 'for what reason did this happen?', sate nanto iú voqiacu de baxi gozaru zo[?] (123) 'what is the name of your guest?', goiô baxi gozaru ca? 'isn't there something of use to you?'

Io and zo strengthen or give cadence to the sentence; e.g., caita zo

\footnotetext{
${ }^{119}$ Cf. Arte $(74,75$, and 75v).

${ }^{120}$ The Dictionarium also has the spelling moxi which suggests that Collado perceived a different vowel quantity than Rodriguez who has moxxi, as does the Vocabulario.

${ }^{121}$ The Latin particle is nonne, which expects an affirmative answer.

${ }^{122}$ Rodriguez, and consequently Doi (Nihon daibunten, p. 449), have xidai for xisai. The original source is the Esopo no Fabulas where on p. 493 the form is xisai.
} 
'he truly wrote,' maitta io 'he certainly came,' sono toqi vare

va ichi dan varui tçucai vo xiraruite gozaru io [. . siaruite ....] (95) 'at that time I was following bad advice.' Bacari means 'only, or in only one way,' sore ni caguitte means 'that only,' core ni caguirazu 'not only this.' Bacari also means 'more or less'; e.g., fiacu bacari 'there were a hundred,' fiacunin bacari corosareta 'about one hundred men were killed.' Nó, nóte, naqu xite, and naqute mean 'without'; e.g., raxxi mo nó 'without reason or order,' cacugo nó 'without any preparation.'

The adverbs of sound (adverbia sonus) are many and vary in accordance with the way that the Japanese perceive the sound. The particle to is added to them; e.g., va va to xite 'vociferously saying wa $w a$, and if they add meqi,u, it means to make even a louder noise; e.g., va meqi,u' 'to shout saying $w a$ '.

\section{The Case Prepositions ${ }^{123}$}

Tame or ni means 'concerning'; ${ }^{124}$ e.g., sonata no tame 'for you (tibi).' It governs the genitive which precedes it; nan no tame 'for what,' nani ni naru ca? 'for what is it?', nani ni xô ca? 'what do you do that for ?', nani no iô ni tatçu ca? (171v) 'for what is it needed, or useful?', maitte no iô va? (130) 'what's the use of going?'

Tai xite means 'on account of' or 'against'; e.g., tengu ni tai xite teqito 'to fight against the devil, or resist him,' Deus ni tai xite cuguio vo coraiuru 'I endure the pain (labor) because of God.' Uie iori also means 'because'; e.g., von jifi no uie iori (167) 'because of his mercy.'

$\mathrm{Ni}$ iotte signifies the reason for which; e.g., Deus iori fito no jento acu ni iotte go fempô vo ataiesaxerareozu [. . ataiesaxerareôzu] (146v) 'God gives to man according to his virtues and vices.' This form is derived from the verb iori, $u$.

$\mathrm{Ni}$ tçuite means 'around, or about' and is derived from the verb tçuqi,u; e.g., core ni ţ̧uite, core ni tçuqi, or core ni tçuqete means 'about that.' Sono gui ni voite va zonjenu (120) 'I do not know anything about this matter,' Vôxe va mottomo naredomo vagami ni totte va canai gatai (120) 'Your Lordship speaks well but what concerns me is that

\footnotetext{
${ }^{123}$ While the material for this section has been drawn from various portions of the Arte, Rodriguez handles the bulk of the matters dealt with here on $106 \mathrm{v}-108 \mathrm{v}$ and $140-148 \mathrm{v}$.

${ }^{124}$ The text is not clear at this point. It reads: Tame. significat ni vel erga: v.g. . . where one would expect: Tame vel ni significat erga: v.g. . . Ötsuka translates this passage as if it were the later, as do I.
} 
it is difficult to do.' Dai quan ni itatte va ichinin bacari sadame io $(120)^{125}$ 'decide that which concerns the steward only.' Itatte and totte $^{126}$ are the gerunds of verbs just as the preceding. They also say Padre coto va 'the things belonging to the priest,' varera coto va 'about my things, or those things which belong to me.' Xitagatte or xitagóte means 'near' and is the gerund of the verb xitagari, $u$ or xitagai,ó. As with the other verbs it governs the dative case; e.g., guioi ni xitagatte or xitagotte 'according to Your Lordship's understanding.' Xidai has the same meaning; e.g., conata xidai 'according to your wishes.' Sometimes it is added to the roots of verbs; e.g., mairi xidai 'according to when he comes, or according to his coming.'

$N i$ indicates the place in which. $N i$ voite has the same meaning but indicates permanence; e.g., fatto va fuximi ni voite vôxeidasareta 'he established the law while he was in Fushimi,' Bungo funai ni itatte 'in the city of Funai in the kingdom of Bungo,' iglesia ni uoru 'he is in church.'

$D e$ indicates the place of an action; e.g., michi de Pedro ni vorta 'I met Peter in the street.' The same particle de, together with vo motte, indicate the instrument with which an action is done; e.g., bo vo motte Pedro vo uchi coroita 'he killed Peter with a stick,' Padre sama catarareta de navo qicoieta 'from what the Reverend Father told me, it became easier to understand,' necqi de xinda 'he died of a fever.'

Cara or iori indicate the place from which; e.g., iglesia cara 'from church.' They also say fune cara maitta 'he came by ship' and cachi cara maitta 'he came on foot.' Fune de maitta is the same as fune cara maitta and fune ni notte maitta. Fana cara me cara miguruxij mono gia 'it is unpleasant to the nose and the eyes.' Iori indicates the place through which; e.g., sama iori faitta 'he entered through the window.'

Tomo ni means 'at the same time'; e.g., sonata to tomo ni mairózu 'I shall go at the same time as you,' mósu to tomo ni 'at the same time as he spoke.'

Ie indicates the place to which; e.g., achi ie mairó 'I shall go directly to court (curia),' miiaco no cata ie noboru 'he went up to court' and also miiaco no fó ie noboru. They also say miiaco no iori,

\footnotetext{
${ }^{125}$ Collado has recast into the colloquial a quote from the Shikimoku. Rodriguez records: Mata daiquanni itatteua ichininnomi sadamubequi nari.

${ }^{128}$ The text reads: itatte $v . g$. totte. ... where the $v . g$. is clearly a misprint of vel.
} 
miiaco sama, or miiaco no gotoqu noboru, but this is not a good way of speaking and is more characteristic of a rustic (rusticus).

$D e$ indicates the material from which; e.g., tçuchi de cavara vo tçuquru 'to make bricks out of earth or mud,' nande core vo tçuquru $c a$ ? 'from what is this made?'

Uie means 'concerning'; e.g., zuibun codomo no wie vo fito ni mo naxi marasuru ióni to cocoro gaqe marasuru 'with great diligence I took care of my sons so as to make them men.' Sonata no fiquan no vo saiban mesare io [. . no uie vo ...] (141) 'take care of your servants.'

Made means 'until'; asu made 'until morning,' inochi vo uxinó made aru mai 'he will not lose his life, or he will not arrive at the loss of his life,' sore made vomoi mo ioranu gui gia 'it will not come to my mind,' cocoro zaxi areba canavanu made mo xei vo iruru 'when something is wished for, one uses his strength up to the point of impossibility,' mósu made mo nai 'it is not necessary to say,' cono tocoro made maitta 'I came to this place.'

\section{Conjunction and Separation ${ }^{127}$}

To means 'and'; e.g., Pedro to juan to Nagasaqi ie ita 'Peter and John went to Nagasaki,' core to, are to vo toru 'I take this and that.' Mo has the same meaning; e.g., Pedro mo juan mo Nagasaqi cara modotta 'Peter and John returned from Nagasaki,' naqu mono mo ari, varó mono mo aru 'there are those who cry and those who laugh. $M o$ is often placed before negative verbs; e.g., nanigoto mo gazaranu $c a$ ? 'is that not something new?'

Mata means 'and,' whether it is found between nouns or verbs. $\mathrm{Ca}$ means 'or'; e.g., Pedro ca; juan ca coi to iie 'tell Peter or John to come.' Arui va also means 'or'; e.g., arui va Pedro, arui va juan 'either Peter or John,' arui va iomu, arui va caqu 'I either read or write.' Moxi va means 'if in fact,' and it is used in the middle of a sentence; e.g., moxi va cane ga nai naraba 'if in fact you were to have no money.'

Mata va is used to bind the sentences more tightly together (ad orationem contexturam). It means 'besides that, or besides'; e.g., arui va iamai ga vocoru ca, mata va isogui no fumi qitaru ca etc.' [. . . ca,' etc.] (135) 'either some sickness occurs, or besides that some urgent letter arrives.'

\footnotetext{
${ }^{127}$ Cf. Arte (130-137).
} 
Xicareba means 'since things are this way,' sari nagara means 'but,' só aru tocoro de means 'since it is thus,' saraba means 'since it is so,' and sareba sareba means 'since then.' Ca? means 'if'; e.g., maitta ca mi io 'see if he came, or went,' maitta ca xiranu 'I don't know if he went.' Iara means 'if,' but distributively (divisive); e.g., fito iara chicuxó iara xiranu 'I don't know if it's man or beast,' nani iara to móxita 'I wonder what he said.'

Some disjunctive and emphatic particles are formed from nanica and tocacu with the addition of other particles; e.g., nani ia ca ia? 'which thing?' The same meaning is expressed by nani iara ca iara? and nanto iara cato iara? Nanto xite, cato xite means 'how,' nanto mo cato mo means 'in no way,' and nani mo ca mo means 'nothing.' Nanigoto mo cagoto mo, mina içtuvari naru zo [. . itçuvari ...] 'when all is said and done they are all lies.' Nani no ca no, and nanto xite, cato xite are ways to excuse oneself. Nani no ca no to iúte means 'saying this and that.' Domo como means 'in whatever way it is,' as does dó xite mo có xite mo. Dó xite có xite means 'doing this and that differently.' Dó xô có xô means 'I shall do this and that.'

Tomo cacumo means 'all the same,' as do toni cacuni, tonimo cacunimo, and totemo cacutemo. Core to ij; care to ij means 'saying this and that, or making excuses.' Care core means 'this and that,' coco caxico means 'here and there (hic and illic).' Vomoxirô, vocaxu ${ }^{12 s}$ [vomoxirovocaxit] is used when accommodating oneself almost to flattery.

If the particle motte is added to the particles catçute, isasaca, tomoni, nani, and nani nani iori $[\ldots$ and nani iori] it adds strength and force; e.g., catçute motte só aru mai 'the situation will not be this way at all.'

\section{Interjections ${ }^{129}$}

Sate, sate sate, [satemo,] and satemo satemo are interjections of admiration; e.g., satemo Deus no voqinaru vonjifi cana 'oh! great mercy of God!'

\footnotetext{
${ }^{128}$ This item is the only one in this paragraph which Rodriguez locs not list as a casane cotoba on $134 \mathrm{v}$ of the Arte. Collado is apparently interpreting this construction as a repetition of two adverbs, as for example coco caxico. If so, the form should be spelled vomoxirô, vocaxiti (if we follow his rule for the formation of adverbs from ij ending adjectives). However, the form which he secms to be recording is more likely the compound adverb which is listed in the Vocabulario as vomoxirovocax in a carefrce manner.' The spelling that we suggest is derived from the attested lexical item without the application of Collado's formational rules.

${ }^{129}$ Cf. Arte $(125-130 v)$.
} 
Avare is the interjection for pity; e.g., avare muţucaxij io no naca cana 'oh! world replete with misery!'

$H a !^{130}$ is the interjection of penetence; e.g., ha faxi demo vomoxiroi ga; tocoro ni iote qicoie canuru [...ni iotte ... $(127 \mathrm{v})$ ' $\mathrm{ah}$, the workmanship of the sound and the harmony of the singing is most graceful, but it is not able to be heard well.'

Iara! is the interjection for joy and pain; e.g., iara iara medeta ia (128) 'oh! how much I rejoice.' Ia is also used; e.g., satemo iiaxii iatçubara ia (129) ${ }^{131}$ 'oh! how vile and despicable,' gongo dódan fuxigui na xisai cana $(128 \mathrm{v})$ 'oh! how rare and ridiculous a reason.' Iei is the interjection of wonder; e.g., iei Padre sama cochi gozaru io 'oh! here is the Reverend Father.'

Hat is the interjection that indicates that one is repentent; e.g., Benqei core vo mite hat coto naxi to zonjite, sono mama niva ni bó vo voraxi, etc. $(127 \mathrm{v})$ 'Benkei seeing this,' etc. ${ }^{132}$

\section{The Syntax and the Cases that are Governed by the Verbs ${ }^{133}$}

The nominative is placed at the beginning of the sentence and the verb at the end: the remaining elements are placed according to the cadence (cadentia) of the sentence; e.g., Pedro va Nagasaqi de xutrai xita iqi iqi ni tçuite juan vo coroita 'Peter killed John because of an argument that took place in Nagasaki.' In certain sentences of serious import a substitute verb (verbum suppositum) is placed after the verb, but this is rare; e.g., tare mo canavanu futari no qimi ni tçucóru coto va $(84)^{134}$ 'no one can serve two masters.' In this sentence the substitute verb is tçucóru coto va. Core ni iote tanomi tatematçuru itçumo virgen [Core ni iotte ...] (84) 'therefore I pray to the ever virgin [Mary].'

Clauses (orationes) in the absolute or permissive subjunctive, infinitive, conditional, and causative are always placed before clauses that are in the indicative or imperative, even if it does not make sense

\footnotetext{
${ }^{130}$ This interjection, together with hat below, are the only uses of initial $h$ found in the description. Rodriguez transcribes the latter item as at or vat (Arte, 127) which suggests a close relationship between the labial and glottal aspirates.

${ }_{1: 11}$ Rodriguez has Benquei satemo yasaxij yatçubaray'a.

1:2 Rodriguez has: . . . nituaye bóno saxivorosu. The entire passage would be, 'Benkei, seeing this, thought, "Oh, this isn't very important," and dropped the stick into the garden." which Rodriguez explains to mean being sorry for not paying sufficient attention to a matter.

${ }^{133}$ The material for this section is derived from various sections in Book II of the Arte.

${ }^{134}$ Matthew, 6:24.
} 
in Latin or any other European language; e.g., achi cara tomeraruru tomo; tomaru na 'do not stay, even if they want you to remain,' sore vo qiitareba, fara vo tatete modotta 'when he heard that, he came back very angry,' taxicani uqetamotta ni iotte coso, móxi ague maraxitare 'I listened carefully, and then I spoke,' faio gozatta raba vo mexi vo xinjô mono vo 'if you had come earlier, I would have offered you food.'

When there are two verbs in the same sentence, the first will be in the gerund form and the other will be in the tense that is required by the sense of the sentence; e.g., core vo totte giqi ni mi ga comono ni vataxe 'take this and give it to my servant at once.'

When there are two or more clauses which have the same subject or tense, only the last verb will be in the tense that is required by the sense of the sentence. The other verbs will be in the root form, while still others will be in the gerund in $e$ form; e.g., tovazunba cotaiezu, voxe raba tçuxxinde qiqi [tovazüba ... vôxe ... qiqe] $(85 \mathrm{v})^{135}$ 'if they don't ask don't answer: if they speak listen carefully,' Deus no vo coto vo macoto ni uqe, go voqite mo camavaide, sono mama inferno ni vochita 'he did not believe in God, and he did not respect His precepts; therefore, he fell into Hell.'

Verbs are always placed in the third person to indicate honor. No one honors himself except the king when he is speaking of himself; e.g., iorocobi ni voboximesu 'I am enjoying it very much.'

When there are many adjectives in a sentence, they will all be in the adverbial form except the last; e.g., qe nangó, iro curô, icanimo utçucuxij mono [qe nagó... . ] $]^{136}$ 'a very beautiful person with long, black hair.'

The particle to is placed before verbs of understanding, believing, and hearing, takes the place of the verb 'to be,' and means 'that'; fito to zonjita 'I thought, or believed that he was a man,' qixó vo jennin to vomovô ca? [qixo ... vomovó ca?] 'shall I believe that you are a saint?' Amata no fito xini no fonovo ni moiuru vo misaxerareô $(20)^{137}$ 'you

\footnotetext{
${ }^{135}$ Rodriguez has the spelling touazumba. In transcribing the form Collado failed to follow the rule he established in his treatment of conditional constructions.

${ }_{1: 66}$ The model for this sentence appears to be Arte (62): Ichidan medzuraxij yenoco, que nagó, uquino gotoqu xiró [sic], me curô, cauo icanimo airaxijuo cureta. If this is the source of Collado's example, he is clcarly demonstrating his sensitivity to the nasalization of such items such as nagó. The Dictionarium under longus has nagai.

1:iz Collado's transcription is unable accurately to express the proper phonological, or morphological, form of shin'i indignation.' He would have been well advised to follow Rodriguez' model and transcribe this item as xiny with the specification that consonant plus $y$ indicates a morphological juncture.
} 
will see many men burning in the flames of indignation.' Here one has replaced to with moiuru vo, which is a substitute verb. When mo is added to to it strongly affirms what is said; e.g., mairó to mo 'I shall certainly go, or I will be going.'

The particle to, in the first meaning, is sometimes replaced by ionni; e.g., agueta ionni gozaru 'they say that he offered it,' ica ionna fito to va xiranu 'I did not know what kind of a man he was.' Sometimes the substantive verb takes the place of the particle to; e.g., mairó de gozatta 'he said that he would come,' xô de va naqeredomo 'although I did not say that I would do it.'

Qiuzo core vo mite, ima vo saigo no coto de areba (97) ${ }^{138}$ 'seeing this, Kiso believed that the hour of death was present, etc.' Here the substantive verb replaces to and serves as an active verb governing the accusative ima vo, which also replaces to. The particles sóna and guena mean 'it seems.' Sorna is added to the roots of verbs; e.g., deqi (63 sóna 'it seems that it is finished.' If a substantive verb is placed after this particle the $a$ is changed to $i$; e.g., deqi sonni gozaru 'it seems that he will finish,' deqi sóni mo zonjenu 'I believe that it will not be finished. ${ }^{139}$ Sóna is also added to adjectives in $i$, and when it is the $i$ is lost; e.g., io sóna 'it seems good, or it seems that it is good,' xigue sóna 'it seems dense,' and aiau sóna 'it seems that I am in danger.' If this particle is added to adjectives in $n a$, the $n a$ is lost; e.g., aqiraca sonna 'it seems that it is clear.'

The particle guena is added to the nouns and verbs previously formed; e.g., maitta guena 'I believe that he has come.' If a substantive verb is added to this particle the a changes to $i$; e.g., maitta gueni gozaru 'I believe that he has come.' Sorna means 'it seems,' and guena means 'I believe,' but either of these forms may occasionally be used in any of the examples given.

When a sentence has two preterits, the first may be in the preterit and the second in the future; e.g., qesa cara só vôxerareta raba mo faia de maraxô 'If you would have said that this morning, I would have already left.'

When reporting what someone else has said, it is said this way;

\footnotetext{
${ }^{138}$ Rodriguez has the spelling Quiso, which agrees with the Amakusaban Heike (p. 239), the ultimate source of the sentence. Collado's spelling in the translation is quiuzo. The Spanish manuscript has Kiso.

${ }^{139}$ One might expect the more literal 'I do not believe that it will be finished,' but Collado has credo quod non finietur.
} 
Padre mósaruru va: iagate sonata ie mairó to mósaruru 'the priest said that he was going to come.' Sometimes when one is excusing himself he will use no in place of to; e.g., asu no, raiguat no, nando to noburu na 'don't spread around that it is tomorrow, next month, or whenever.'

When $v o$ follows $n$ it loses its $v$; e.g., go von o uqetatemaţutta 'I received benefits. ${ }^{140}$

Adverbs are always placed before their verbs except for the adverbs of time which are placed at the beginning of the sentence; e.g., sore vo qijte iccó xicari maraxita 'hearing that he was very angry,' qiô nen espana cara vatatta toqi [qio nen ...] 'when I sailed from Spain last year.' Each verb requires before it a subject in the nominative case, either expressed or understood; e.g., vare iqe or iqe 'come!', where the vare is understood. In some sentences this rule is not respected; e.g., xisai voba core ni mósare maraxozu [.. maraxôzu] 'he will explain, or give the reason for this.' In the following case we do not see the nominative, but rather are ni va, which is in the dative or ablative; are ni va, navo voixri atta [. . voxiri atta] 'he knows better.' In this sentence the are ni va ought to be in the nominative. Cacaru vo ni va cogane no cusari vo icusugi mo ţ̧uqeta dógu de gozaru (138v) 'for a necklace (torques) he had a chain of gold with many links.'

Core ni va gozonji aru mai 'Your Lordship does not know about this.' Here the core ni va replaces the accusative which is governed by zonji,uru.

The impersonal verb or the infinitive requires a nominative before it; e.g., Pedro va maitta to mósu 'they say that Peter came.'

The verb iri, u, which means 'to need,' governs two nominatives, one for the thing and the other for the person in need; e.g., vatacuxi va cono cane ga iru 'I need, or I have a necessity for this money.' It also governs the dative for the person; e.g., sono tame ni va cane ga iranu 'he does not need any gold, or money.'

The active verb requires the accusative before it; e.g., cane vo motanu 'I have no money.'

Certain cobita or coie nouns, as we have said above, are borrowed from Chinese and govern the same cases as the Japanese verbs to which

\footnotetext{
${ }^{140}$ This rulc, which might more appropriately have been included with the phonology, is not followed in Collado's description, with the possible exception of p. 48 where the same construction is apparently used.
} 
they correspond; e.g., niva vo qenbut no aida ni mexi vo coxiraie io 'prepare the food while we visit the garden.' The noun qenbut requires the accusative niva vo. The same is true with fito ni guenzan suru (97) which is like fito ni vó 'I meet the man.' The guenzan governs the dative just as does the verb ai,ó.

When a borrowed word (vocabulum cobitum $)^{141}$ is a compound of two elements it is possible to determine if it is a verb by seeing if the first part has the meaning of a verb; e.g., jóten is a verb which means 'to ascend to heaven' with the jor meaning to 'go up.' Tenjó is a noun in which the $j o$ is placed after the ten and means 'heaven.'

The passive verb has the ablative for its agent (persona agente); e.g., Pedro cara corosareta 'he was killed by Peter,' but it is better that it govern the dative; e.g., Pedro ni corosareta, or Pedro va nusubito ni cane vo torareta 'Peter had his money stolen by thieves.'

There are also certain neutral verbs which govern the accusative as if they were active verbs; e.g., xiqitai vo fanaruru 'to depart from the body, or to die,' axi vo vazzuró 'to have a pain in the foot.' This is also true for nigue,uru 'to escape,' nogare,uru 'to evade,' de,uru 'to go out,' noqe,uru 'to retreat,' tovori, $u$ 'to go across,' nori, $u$ 'to sail,' as in caixó vo noru 'I sail the sea,' iuqi,u 'to walk,' as in michi vo iuqu 'I walk the streets,' vovari, $u$ 'to finish,' mairi, $u$ as in xogui vo mairu 'I play chess (tabula laterucularia),' iorocobi, $u$ as in cocoro vo iorocobu 'I gladden the heart,' abi,uru, as in mizzu vo abiru' ${ }^{142}$ 'I wash myself with water, or I pour water on myself,' avaremi, $u$ 'I am sad,' canaximi,u 'I am unhappy,' coie,uru 'to cross over,' fabacari, 'u 'to be shy,' facarai,ŏ 'to take care of,' faxiri,u 'to sail,' as in caixó, vo, faxiru [caixó vo faxiru] 'I sail the sea,' fagi,zzuru 'to be ashamed,' fedate,ţuru 'to separate,' fe,uru 'to spend,' as in ficazu vo furu 'I spend many days,' [fumaie,uru 'to be based on,' as in] dori vo fumaiuru 'to be based on reason, or to have reason as a basis,' itami, $u$ 'to be sick,' mavari, $u$ 'to go around,' as in cono cotovari vo móxi mavatta 'he goes around and spreads the news here and there,' meguri,u has the same meaning, nagusami,u 'to please,' as in cocoro vo nagusamu 'I make the heart

\footnotetext{
${ }^{141}$ Collado here demonstrates the absorbitive capacity of Latin as he creates an accusative singular adjective from the past attributive of the verb kobu.

${ }_{142}$ The use of abiru, where one would expect aburu, may be a simple typographical error or evidence that Collado accepted the shift from $n i-d a n$ to ichi-dan katsuyo as unworthy of notice. Rodriguez (Arte, 101v) has midzumio aburu.
} 
happy,' naqi,u 'to weep,' tasucari,u 'to be saved,' as in inochi vo tasucaru 'I am saved from the dangers of life,' or goxó vo tasucaru 'to be saved for a future life,' tachi,ţu 'to go away from,' as in tocoro vo tatçu 'I go away from this place,' tomurai,ó 'to make a funeral for the dead,' ucagai,ó 'to inquire with hesitation,' voximi, $u$ 'to value,' urami, $u$ 'to enquire,' xinobi, $u$ 'to wait in hiding, almost insidiously,' as in fito no me vo xinobu 'I am careful lest someone see me.' ${ }^{143}$ A few of these verbs which require the accusative of location admit to the use of the ablative with the particles cara or iori; e.g., tocoro vo tatçu is the same as tocoro iori tatçu 'I leave the place.'

There are some active verbs which require two accusative cases; e.g., fori,u, daxi,u, fanaxi,u, tate,tçuru. For example, Pedro vo soco vo voi idaita 'they led Peter away from that place.' It is possible that it governs the ablative of location; e.g., Pedro vo soco cara voi daita [. . voi idaita]. Some take either the dative or the accusative; e.g., fito vo, or fito ni fanare,uru 'to go away from the men,' Deus vo, or Deus ni somuqi, $u$ 'to offend God.' Verbs of this kind are generally verbs of fearing, offending, or going away. ${ }^{144}$

Many verbs of helping, harming, damning, obeying, recognizing as superior or inferior, being subjugated, being victorious, and similar verbs govern the dative; e.g., chiie saicacu ionni coieta 'he is superior to others in wisdom and industry. ${ }^{.145}$

Verbs of giving, promising, and the like, govern the accusative for the thing and the dative for the person; e.g., fito ni cane vo cururu 'to give money to someone. ${ }^{146}$

There are many verbs which permit before themselves the roots of other verbs without change, letting the roots take on the function of an infinitive; e.g., qiqi fajime,uru 'to begin to hear.' Some of these verbs are: nare,uru 'to become accustomed,' tçuqe,uru with the same (66 meaning, fate,tçuru 'to finish,' narai,ó 'to learn,' some,uru 'to begin,' todoqe, uru 'to continue,' ate, tçuru 'to direct,' atari,u 'to find by chance,' vaqe,uru 'to divide,' cane,uru 'to be able to do with difficulty,' soconai,ó 'to be wrong,' sumaxi,u 'to finish,' sugoxi,u 'to exceed,' fague maxi,u

\footnotetext{
${ }^{143}$ This list is derived from the Arte $(101 \mathrm{v}-102 \mathrm{v})$. From abi,uru on, the list is in the same order as that made by Rodriguez. Fanarum, zzuru, nosquru, noru, vovaru, and mairu are Collado's contributions.

${ }^{144}$ Cf. Arte (101v).

${ }^{145}$ Cf. Arte $(100)$.

${ }^{146}$ Cf. Arte (98).
} 
'to work much and intensely,' aqi,u 'to become bored,' tai 'to want,' and tor mo nai 'to not want." ${ }^{\prime 47}$ If the roots of verbs are placed before certain adjectives ending in $i$, they form a kind of supine in $T u$; e.g., iomi iasui (92) 'easy to read (facile lectu),' etc.

A numeral, if a substantive noun is placed after it, must be in the genitive case; e.g., fitotçu no toga 'one sin.' The same is true with the particle fodo when it means 'all'; e.g., aru fodo no fito 'how so ever many.' The same is true with iori; e.g., Nanban iori no mono 'things from Europe.' But this is a relative formation (relatiuum). The genitive is also required with nouns that mean much or little; e.g., amata no fito 'many men.' These nouns are; bechi 'other,' fon 'one's own,' cazucazu 'many,' sama zama 'many ways.' Iro iro 'much' is the same as iorozzu and izzure. Issai means 'all,' as does vono vono, cotogotoqu, and reqi reqi for a noble person, igue 'that which follows,' nocori 'that which remains,' itçumo 'always,' itçumo no coto 'that which always is,' tçune 'usual,' ima 'now.' Isasaca means 'a little,' as does soto or sucoxi, xotocu 'natural,' sono foca 'others. ${ }^{\text {'148 }}$ These nouns are in the genitive if they are followed by a substantive noun, but when they are not followed by a noun they must be taken as adjectives. If they are followed by a verb rather than a noun, they do not require the genitive; e.g., iorozzu dancó xite iocarŏ 'it will be good if you all confer.'

\section{Japanese Arithmetic and Numerical Matters Concerning Which Much Painful Labor Is Required}

There are two ways to count in Japanese. ${ }^{149}$ The first is with the ordinary numerals which are called iomi. With these one is able to count to ten; e.g., fitotçu means 'one,' which is also used to (67 say 'a little,' as in saqe fitotçu nomaxite tamóre 'give me a little sake to drink.' Futatçu means 'two,' mitçu 'three,' iotçu 'four,' itçutçu 'five,' mutçu 'six,' nanatçu 'seven,' iaţ̧u 'eight,' coconotçu 'nine,' and tovo

\footnotetext{
${ }^{147}$ Cf. Arte (104).

${ }^{148}$ Cf. Arte (64 and 79).

${ }^{149}$ The material presented in this section is glcaned from the exhaustive treatment of the numerical system which makes up the last 20 leaves of Rodriguez' grammar.
} 
'ten.' Icutçu means 'what?' and is used when one does not have the proper number.

The second way of counting is with the coie vocables which are borrowed from Chinese. These numbers are not used by themselves to count to ten; but are rather used when counting things which are represented by Chinese, and not Japanese vocables. These bound numerals (termini numerales) are: ichi 'one,' $n i$ 'two,' san 'three,' $x i$ 'four,' go 'five,' rocu 'six,' xichi 'seven,' fachi 'eight,' cu 'nine,' jú 'ten.' The numbers eleven and above are made by joining these numbers together. Thus, 'eleven' is jüichi; jüni is 'twelve,' júsan 'thirteen,' júcu 'ninteen.' The tens are obtained by placing one of the numbers in front of ten; e.g., nijú 'twenty,' sanjú 'thirty', sanjǔichi 'thirty-one,' cuju 'ninety.' Fiacu means 'hundred,' fiacu ichi 'one hundred and one,' fiacu jü 'one hundred and ten,' fiacu sanjü 'one hundred and thirty,' ni fiacu 'two hundred,' sambiacu 'three hundred.' Xen means 'thousand,' and xen roppiacu sanjü ichi is 'sixteen thirty-one.'

By placing the Japanese numerals in front of Japanese vocables, which are called iomi, and by removing the $t_{\zeta} u$ of the aforementioned numbers before they are joined to nouns or verb stems, one is able to enumerate those things which are indicated by the vocable; e.g., fito cotoba 'one word,' futa cotovari 'two reasons,' mi ami 'three nets, or three casts of the net,' iocama 'to bake something four times in an oven,' itçu caqe 'five attacks,' $m u$ casane 'six robes, or covers,' nana catana 'seven wounds by a sword,' ia catague 'eight loads,' cu cavari ${ }^{150}$ 'nine changes,' to cusa 'ten varieties.' Above the number ten this way of counting is not used, instead they say iro juichi or júichi no iro for 'eleven colors.' The interrogative is icutçu. If the thing being questioned is placed after the interrogative the particle no is added; e.g., itçucu no qi zo [icutçu ...] 'how many trees are there?' To such a question the answer is futatçu 'two,' mitçu 'three,' etc. If the tçu is removed from icutçu, one may place it in front of the thing being asked about; e.g., icu tocoro 'how many places?' icu toqi 'how many hours?'; also fito fanaxi 'one sermon, or conversation,' futa sugi 'two treads,' io te 'four hands, as in a fight,' itçu ţ̧ubu 'five grains,' mu tocoro 'six places,' ia mavari 'six [eight] circuits,' cu ninai 'nine loads, carried in

\footnotetext{
${ }^{150}$ This compound does not follow the rule, since $c u$ is not a iomi numeral. See also cu ninai below.
} 
the Japanese fashion on a stick with the load in front,' to vatari 'ten crossings.' It is possible to count the same thing in different ways. Thus, mu tocoro is also mutçu no tocoro and tocoro mutç 'six places.' Fito ie means 'one plain thing,' futa ie 'doubled, or duplicate,' mi ie 'triplicate,' etc. In the same way one may add Chinese numerals to Chinese vocables, or coie. Usually in this way of counting a [phonetic] change occurs in either the number or the thing counted. Sometimes this change is in the first part, sometimes in the second, and at other times in both. This is particularly true with the first, second, third, sixth, tenth, and one hundredth numbers. With the items below, if nothing is noted, it is an indication that nothing is changed.

When asking about men one says icutari? 'how many men?' The response is made by adding nin to the Chinese numeral; e.g., ichi nin 'one man,' ni nin 'two men,' iottari 'four men'; this is because xinin means 'dead person.'

When asking about days one says icca 'how many days?' The response is fi fitoi, ${ }^{151}$ because ichi nichi means 'one entire solar day,' futçuca 'two days,' micca 'three days,' iocca 'four days,' itçuca 'five days,' muica 'six days,' nanuca 'seven days,' ióca 'eight days,' coconoca 'nine days,' toca ${ }^{152}$ 'ten days,' fatçuca 'twenty days.' The remaining days are counted with coie numerals.

When counting nights $i a$ is added to the coie numerals; e.g., ichi ia 'one night,' ni ia 'two nights,' etc. It is also possible to add io which means 'night' in Japanese to the iomi numeral; e.g., icu io 'how many nights?' futa io 'two nights,' nana io 'seven nights,' etc.

When enumerating the months of the year guat is added to the coie numeral, with the exception that the first month is called xóguat. The second is niguat, the third is saguat, ${ }^{153}$ the fourth is xiguat, the eleventh is ximotçuqi, and the twelfth and last is xi vasu. When counting months the $t c ̧ u$ is removed from the iomi numeral and the word tçuqi, which means 'month,' is added. Icutçuqi? means 'how many months.' In response one says fitotçuqi 'one month,' up to ten which is totçuqi, and from there on one counts with coie numerals; e.g., júichiguat 'eleven months.' If one wants to ask what month it is,

\footnotetext{
${ }^{151}$ Rodriguez has fitoi or fifitoi (Arte, 228v).

${ }^{152}$ While this form fits the general rule for combining counters and days, Rodriguez (Arte, 228v) has tóca, which is a misprint for tôca, cf. Doi, Daibunten, p. 818.

${ }^{153}$ Spelled with a tilda, sãguat, as are all the other forms before guat.
} 
January, February, one says nanguat. The first month of the Japanese year is March.

In the enumeration of the years nen is placed after the coie numeral. In asking how many, nen [nan] is placed before nen; e.g., nannen 'how many years?' In response one says ichinen 'one year,' ionen 'four years,' sanganen 'three years,' só ionen ${ }^{154}$ 'three or four years,' só xijúnen 'thirty or forty years,' fatachi means 'twenty years of age,' as does nijunen, nijü no toxi, or toxi niju. They ask with icutoxi or toxi icuţu 'how old are you.' They count the age of men and animals such as cattle and horses by adding sai to the coie numeral; e.g., issai 'one,' nisai 'two,' sanzai 'three.'

In counting turns (visis) do is added to the coie numerals; e.g., nando 'how many times,' ichido 'once,' iodo 'four times,' godo 'five times,' sai san 'twice or thrice.'

In the enumeration of ships sô is placed after the coie numeral; e.g., nanzo [nanzô] 'how many ships,' to which one answers issô 'one ship,' niso [nisô] 'two,' sanzô 'three,' fassô 'eight,' jússô [jissô] 'ten.'

Ichiren 'one string,' niren 'two,' saren 'three,' as in figs or pearls.

When enumerating sermons, homilies (tractatus), or repetitions of things, fen is placed after the numeral; e.g., ippen 'one sermon,' nifen 'two,' sanben 'three,' ave maria fiacu gojippen 'one hundred and fifty Hail Mary's.'

In counting gold currency momme is placed after the numeral; e.g., ichi momme 'one momme,' ni momme 'two,' san mome [san momme] 'three.' When a momme is divided into tenths it is called an ippun [fun]. Thus, ippun means one tenth part of a momme, nifun means ' 'two tenths,' gofun means half the basic unit (media dragma), roppun means 'six tenths of a momme.'

When the tenth part of a momme is divided again into ten parts it is counted as ichirin, nirin, sarin, iorin, gorin, rocurin, xichirin, fachirin, and curin. Then comes ippun, which is one tenth of a momme. Fiacu me means 'one hundred momme,' fiacu ichi momme 'one hundred and one,' icquan me means 'one thousand momme,' jicquanme means 'ten thousand.' There are other coins of silver which are counted by placing mai or mon after the numeral; e.g., ichi mon means one of

\footnotetext{
${ }^{154}$ For the só and sa allomorph of san cf. Arte (173v).
} 
that unit, ni mon is 'two,' San mai is three hundred mon. They no longer produce a coin which is one half of the gold coin, but one thousand of these coins make icquan, while jicquan is 'ten thousand quan. ${ }^{155}$

Core va ica fodo ni suru 'how much is this worth?' or ica fodo ni uru 'at what price will you sell this?' Ni momme suru 'I consider it worth two momme,' or ni momme ni iasui 'I can sell this for more than two momme, or at two momme this is cheap.'

The enumeration of liquid measurements is done by placing the particle $x \hat{o}$ in front of the liquid quantity; e.g., ixxo [ixxô] 'one $x \hat{o}$,' nixo [nixô] 'two,' sango [sanjô] 'three.' Ten $x \hat{o}$ are itto which is the particle to placed after the numeral; nito means 'twenty xô,' sando 'thirty.' For one tenth of a $x \hat{o}$ one places the particle go after the numeral; e.g., Ichigo 'one go,' nigo 'two,' sango 'three,' ixxô gogo 'one and one half xô.' Fatto is eighty xô. One hundred xô make ichi cocu. By placing the cocu after numerals one obtains ni cocu 'two hundred xô,' sangocu 'three hundred,' jiccocu 'one thousand,' xencocu 'ten thousand,' ichi mangocu 'one hundred thousand.'

The enumeration of the measurements of human height is achieved by placing fito [firo] after the iomi numerals; e.g., fito firo 'one firo,' futa firo 'two,' jippiro 'ten.' The measurement of a span (palmus) is made by adding xacu to the coie numerals; e.g., ixxacu 'one span, or three spans by the Spanish measuring system, ${ }^{156}$ sanjaku 'three.' Goxacu is the same as fito firo which is a measurement we have referred to before. Six xacu make up a measurement called icqen 'one qen,' nicqen [niqen] 'two,' jicqen 'ten,' and sanguen 'three.' From sixty of these measurements one makes a measurement called icchó, that is 'one mountain path,' nicchó [nichó] 'two,' jichió [jicchó] 'ten,' sangió 'three.' From sixty-three [thirty-six] chô, as measured in the northern part of Japan, one obtains ichiri which is one league or one miliar. One enumerates by adding $r i$ to the coie numerals; e.g., niri 'two,' sanri 'three,' gori 'five,' juiri 'ten'; iori is 'four,' because xiri means anus. ${ }^{157}$ Fan michi

\footnotetext{
${ }^{155}$ Rodriguez gives the following equivalents in the monetary system on $217-217 \mathrm{v}$ of the Arte: ... ten Rin in one Fun, ten Fun in one Momme, one thousand Momme in one Quamme.

${ }_{158}$ The text is confused at this point. It runs: Ixxacu, unus palmus seu tertia quam Hispania vocant sanjacu. tres, ... .

${ }^{157}$ The text has culus 'posterior,' but the crrata changes the word to anus. The original seems closer to the Japanese.
} 
means 'a half of a league.' They say; ioco fan miqi tate ichiri [ . . michi ...] 'a half a $r i$ wide and one $r i$ long,' faba icqen 'the width is one qen,' iofó futa firo 'two hiro on all sides.'

The cardinal numbers first, second, etc. are made by adding ban to the coie numerals; e.g., ichi ban 'first,' ni ban 'second.' To these are also added $m e$, as said before; e.g., xi ban me 'forth.' One may also make the cardinal numbers by placing dai in front of the coie numerals; e.g., daiichi 'first,' daini 'second,' etc.

The enumeration of multiples is done by adding bai to the numbers; e.g., ichibai 'double,' nibai 'triple,' sanbai 'quadruple,' ${ }^{15 s}$ fiacu zobai 'one hundred fold.'

The enumeration of the parts from the whole is done by placing buichi after the numeral; e.g., ni buichi 'one from two parts,' san buichi 'one from three parts.'

To indicate one tenth vari is placed after the numeral; e.g., ichi vari 'one from ten parts,' xi vari gobu 'four and one half from ten parts.' $J$ u buichi is the same as ichi vari.

The enumeration of oars, muskets, and long things made of wood is done by placing chó after the numerals; e.g., icchó 'one oar,' nichó 'two,' sangió 'three,' jichó [jicchó] 'ten.'

The enumeration of fish and fire wood is done by placing con after the numerals ${ }^{159}$ e.g., iccon, 'one fish,' sangon 'three,' jiccon 'ten,' fiaccon 'one hundred,' fiacu gojù sangon 'one hundred and fifty-three.' This is the amount Saint Peter caught, and even though he caught that number the net did not tear.

The enumeration of leaves of paper and sheets of gold, etc. is done by placing mai after the numeral; e.g., ichimai 'one leaf,' cami gomai 'five leaves of paper.'

The enumeration of the stories of a house is done by placing cai after the numeral; e.g., nicai 'the first floor,' sangai 'the second,' xigai 'the third,' gocai 'the fourth,' when counted as in a house in Madrid.

The enumeration of utensils and cups for drinking is done by placing fai after the numeral; e.g., ippai 'one drink, or one draught,' nifai 'two,' sanbai 'three,' jippai 'ten.'

\footnotetext{
${ }^{158}$ The examples here lag one behind the glosses.

${ }^{159}$ Here and elsewhere Collado combines homophonous enumerators which Rodriguez keeps distinct. Cf. Arte (220-223v) for an extensive list of enumerators.
} 
The enumeration of rolls of silk or the like is done by placing tan after the numeral; e.g., ittan 'one roll,' nitan 'two,' sandan 'three,' jittan 'ten.' Xichitan bune is a ship with a sail seven tan wide.

This is also said by adding mai to the numeral; e.g., gomai 'five,' as in gomai bune 'a ship having a sail five mai wide.'

The enumeration of four-footed animals is done by placing fiqi after the numeral; e.g., ippiqi 'one animal,' nifiqi 'two,' sanbiqi 'three,' roppiqi 'six,' jippiqi 'ten,' fiappiqi 'one hundred,' xenbiqi 'one thousand.'

The enumeration of images, pictures, and medicines is done by placing fucu after the numeral; e.g., ippucu 'one item,' nifucu 'two,' sanbucu 'three,' ropрuси 'six,' jiрриси 'ten.' Needles are also counted this way.

The enumeration of pounds (libra) is done by placing qin after the numeral; e.g., icqin 'one pound,' niqin 'two,' sanguin 'three,' rocqin 'six,' jicqin 'ten,' fiacqin 'one hundred,' xenqin 'one thousand.'

The enumeration of masses and congregations of men is done by placing $z a$ after the numeral; e.g., ichiza 'one congregation,' niza 'two,' sanza 'three,' jüza, or better toza 'ten.'

The enumeration of sacks of rice, wheat, and the like, is done by placing fiô after the numeral; e.g., ippiô 'one sack,' nifiô 'two,' sanbiô 'three,' xifio [xifiô] 'four,' roppio [roppiô] 'six,' jippio [jippiô] 'ten,' fiappio [fiappiô] 'one hundred,' xembiô [xenbiô] 'one thousand.'

The enumeration of pieces of wood, reeds, and needles is done by placing fon after the numeral; e.g., ippon 'one item,' nifon 'two,' sanbon 'three,' roppon 'six,' jippon 'ten,' fiappon 'one hundred,' xenbon 'one thousand.'

The enumeration of bundles (fasciculus) is done by placing $v a$ after the numeral; e.g., ichiva 'one bundle,' niva 'two,' sanba 'three,' jippa 'ten,' juichiva 'eleven,' ni jippa 'twenty.'

The enumeration of burdens or the packs that horses carry is done by placing só after the numeral; e.g., issó 'one burden,' nisó 'two,' sanzó 'three,' jissó 'ten.' In the same way one counts those furnishings called bióbu; two or a pair from a set is called issó, etc.

The enumeration of that which in the vernacular is called a quire of paper (mano de papel) is done by placing giô after the numeral; e.g., ichigio [ichigiô] 'one quire,' nigio [nigiô] 'two,' sangiô 'three,' so on 
to ten. Units of ten are counted by adding socu to the numeral; e.g., issocu 'ten quires,' or what in the vernacular is called a half ream (media resma),' nisocu 'twenty, or an entire ream.' With this particle socu added to numerals one also counts pairs of shoes; e.g., issocu 'a pair of shoes.'

The enumeration of substance (substantia) is done by placing tai after the numeral; e.g., ittai 'one substance,' nitai 'two,' sandai 'three.' Deus no von tocoro va goittai de gozaru 'God as God is of one substance and one essence.'

The enumeration of the divisions in a writing (capitulum) is done by placing cagiô after the numeral; e.g., iccagiô 'one chapter,' nicagio [nicagiô] 'two,' sangagio [sangagiô] 'three,' roccagio [roccagiô] 'six,' fiaccagio [fiaccagiô] 'one hundred.'

The enumeration of drops is done by placing teqi after the numeral; e.g., itteqi 'one drop,' jitteqi 'ten.' The same meaning is obtained by adding xizzucu to the iomi numeral; e.g., fito xizzucu 'one drop,' etc. In this case the $t_{c} u$ must be removed from the numeral.

The enumeration of the pairs of small sticks (paxillus) with which they eat is done by placing tçui after the numeral; e.g., itçui [ittçui] 'one pair,' jittçui 'ten.'

The enumeration of bundles is done by placing $c a$ after the numeral; e.g., icca 'one bundle,' nica 'two,' sanga 'three.'

The enumeration of books is done by placing quan after the numeral; e.g., icquan 'one book,' niquan 'two,' sanguan 'three,' roquan [rocquan] 'six,' jiquan [jicquan] 'ten.'

With the interrogative nan, when it is placed before one of these nouns, it changes it in the same way as does the number three; e.g., ano mmadomo va nanbiki zo? 'how many horses are there?'

The enumeration of kingdoms (regnum) is done by placing cacocu after the numeral; e.g., iccacocu 'one kingdom,' nicacocu 'two,' sangaсоси 'three,' jiccacocu 'ten.' Kingdoms are divided into provinces or districts called gun, and this word also is placed after the numeral; e.g., ichigun 'one province,' nigun 'two,' sangun 'three,' etc.

Sermons and exhortations are enumerated by placing dan after the numeral; ichidan 'one sermon, or assembly.' Words are enumerated by 
placing gon or guen after the numeral; e.g., ichigon 'one word,' sanguen 'three words.'

Placing the particle zzutçu after either coie or iomi numerals gives the meaning of 'each'; e.g., ichinin ni uxi sanbiki zzutçu vo toraxeta 'he let the men have three oxen each,' ichinin zzutçu saqe sanbai zzutçu vo nomareta 'each man drank three sake each.'

In speaking of two or three things separately, they join the two numbers; e.g., xigonin 'four or five men,' from which others may be copied.

The honorific particles are four; vo, von, go, and $m i .^{160}$ The first two are joined to iomi vocables. The last two are joined to coie, or Chinese vocables. The last is the most honorific and is used when speaking of things divine; e.g., midexi tachi 'disciples of Christ the Lord,' goichinin vocoite cudasarei 'please send one from among the Lords.'

The words which follow have honorific particles that have been added by the speaker. However, the honor is shown to the person addressed or to those related to him; e.g., go focô [go fôcô] 'a duty,' von furu mai 'a banquet,' von cotoba 'a word, or a sermon,' von mono gatari 'a conversation,' von natçucaxij or von nocori vovoi which mean the same as what the Portuguese call saudades (nostalgia) and the Spanish call carino (affection), von tori avaxe 'intercession,' von mi mai 'a visit,' von cha 'that which one drinks when they invite you,' go dancó 'a consultation or congregation for the purpose of obtaining advice,' von rei 'an act of gratitude,' von busata 'a lapse of good manners,' vo motenaxi 'to treat well and elegantly,' go chiso [go chisô] 'esteem,' go iqen 'an opinion,' e.g., fabacari nagara go iqen vo móxitai 'forgive me but I would like to give you some advice,' etc.

\section{Some Rules on the Conjugation of the Verb in the Written Language}

If the final $u$ is removed from the negative present it becomes an affirmative verb; e.g., oracio vo tçutomen toqi va 'when I say my prayers,'

${ }^{100}$ Cf. Arte (159-159v). 
xosa no tçutomen tame ni va 'in order to execute the work,' michibiqi tamavan to voboximexi 'thinking of leading forth." ${ }^{161}$

For the affirmative future beqi is added to the affirmative form with the $r u$ removed; for the future negative becarazu is added to the affirmative form; e.g., mósu beqi 'you will speak,' mósu becarazu 'you will not speak.' When the sentence ends in the future, beqi is changed to bexi.

The infinitive for the future is formed by adding coto to the future tense; e.g., iomu beqi coto. The subjunctive is formed by adding qereba to the root of the verb; e.g., sugure qereba.

The gerund in $D o$ is formed by adding te to the root of the verb; e.g., qiqi tamaite.

The substantive verb in the written language is nari,u or qeri,u. If it comes at the end of the sentence it takes the root form; ${ }^{162}$ e.g., sadame naqi io no ixei nari 'it is the dignity of a world without stability.'

The preterit is formed by adding ari,u $[$ tari,u] to the root; e.g., suguretaru. If the form comes at the end of a sentence ari,u (75 $[$ tari, $u]$ is retained in the root form; e.g., suguretari.

The pluperfect is formed by placing nari after the present tense; e.g., ague tamó nari 'they had shown respect.'

Even though there are other rules for the written language, if the reader knows Japanese well enough to read books, he will be able to progress in the language without difficulty.

\section{PRAISE BE TO GOD}

${ }^{101}$ This rule, apparently an invention of Collado's, has no precedent in Rodriguez or in linguistic derivation. The $n$ in this construction is the contracted form of the classical $m u$, the source for what Collado calls the future.

${ }^{102}$ These forms might better have been presented as nari, $i$ and qeri, $i$ to indicate that the sentence-ending forms are nari and qeri. 



\section{Works Consulted}

Alvarez, Manuel (Emmanuel Alvarus), De Institutione Grammatica, Libri III, Lisbon, 1572. (Also Amakusa, 1594. Cf. Laures \#14.)

Collado, Diego, O.P., Ars Grammaticae Iaponicae Linguae, Rome, 1632. (Trans. by Ōtsuka Takanobu as Koiyaado-chö Nippon bunten, 1934 and revised as Koryaado Nihon bunten, 1957. Cf. Laures \#54.)

Collado, Diego, O.P., Dictionarium sive Thesauri Linguae Iaponicae Compendium, Rome, 1632. (Edited by Ōtsuka Mitsunobu as Koryaado Ra-SuNichi jiten, 1966. Cf. Laures \#56.)

Collado, Diego, O.P., Niffon no cotõba ni yô confesion, Rome, 1632. (Transcribed by Ōtsuka Mitsunobu as Koryaado zangeroku, 1957. Cf. Laures \#56.)

Doi Tadao 去忠生, Kirishitan gogaku no kenkyü 吉利支打語 学の研究, Tokyo, 1971 .

Doi Tadao土井忠生, “Koryaado Nihon bunten no seiritsuコリヤ一ト 日本文典 の成立,” Nihon gogaku shinkōinkai kenyü hōkoku, \#3, 1941.

Doi Tadao土抹忠生, ed., Nippo jisho 日葡辞書, Tokyo, 1960. (Japanese edition of the Vocabulario.)

Doi Tadao 土井忠生, trs., Rodorigesu Nihon daibuntenロドリゲス 日本大文典 Tokyo, 1955. (Trans. of Rodriguez' Arte.)

Fukushima Kunimichi 福息邦道, Kirishitan Shiryo to kokugo kenkyū キリシタン資料と国語研究, Tokyo, 1973.

Hashimoto Shinkichi 橋本進吉, Kirishitan kyögi no kenkyü 吉利支丹教義の研究, Tokyo, 1928 .

Iwai Yoshio 岩井良雄, Nihongohō-shi: Muromachi-jidai hen 日本語法处 Tokyo, 1973，

Laures, Johannes, S.J., Kirishitan Bunko, Tokyo, 1957.

Lebrija, Antonio (Antonius Nebrissensis), Introductiones Latinae, Salamanca, 1481.

Moran, Joseph F., A Commentary on the Arte Breve da Lingoa Iapoa of João Rodriguez, S.J.: With Particular Reference to Pronunciation, Unpublished doctoral thesis, Oxford, 1971.

Ōtomo Shin'ichi 大友信一, Muromachi-jidai no kokugo-onsei no kenkyū 空町時代の国語音声の研究, Tokyo, 1963.

Ōtsuka Mitsunobu 大塚光信, ed., Koryaado Ra-Su-Nichi jiten

コリヤード羅西日樀典, Tokyo, 1966. (Japanese edition of Collado's Dictionarium.)

Ōtsuka Mitsunobu 大场光信, ed., Koryaado zangerokuコリヤ—ド

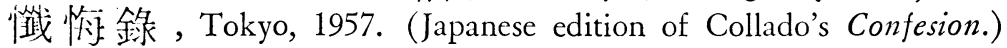


Ōtsuka Takanobu 大场商信, tr., Koiyaado-chō Nihongo bunten コイヤ一ド著日本語文典, Tokyo, 1934. (Revised as Koryaado Nihon buntenコリヤ一ド日本 文典, Tokyo, 1957. Translation of Collado’s Ars Grammaticae.)

Rodriguez, João, S.J., Arte Breve da Lingoa Iapoa, Macao, 1620. (Cf. Laures \#35.)

Rodriguez, João, S.J., Arte da Lingoa de Iapam, Nagasaki, 1604-1608. (Translated by Doi Tadao as Rodorigesu Nihon daibunten, 1955. Cf. Laures \#28.)

Rodriguez, João, S.J., ed., Vocabulario da Lingoa de Iapam, Nagasaki, 1603-1604. (Edited by Doi Tadao as Nippo Jisho, 1960. Cf. Laures \#27.)

Thurot, Charles, Extraits de divers manuscrits Latins pour servir a l'historie des doctrines grammaticales au moyen-age, Paris, 1869.

Yuzawa Kōkichirō 湯澤幸吉郎, Muromachi-jidai gengo no Kenkyū 突町時代言話の研岔, Tokyo, 1958. 


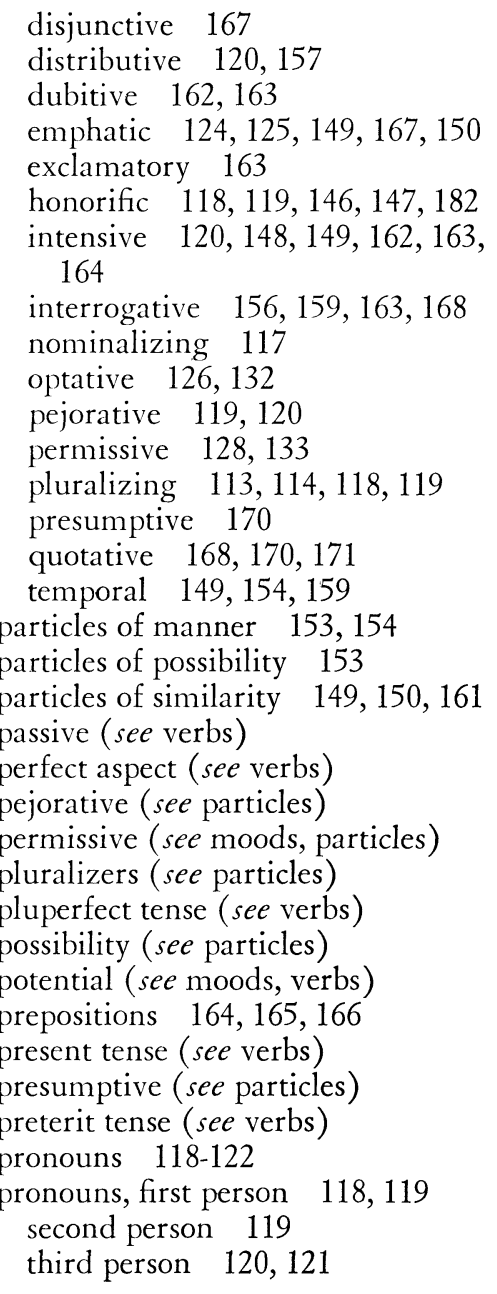

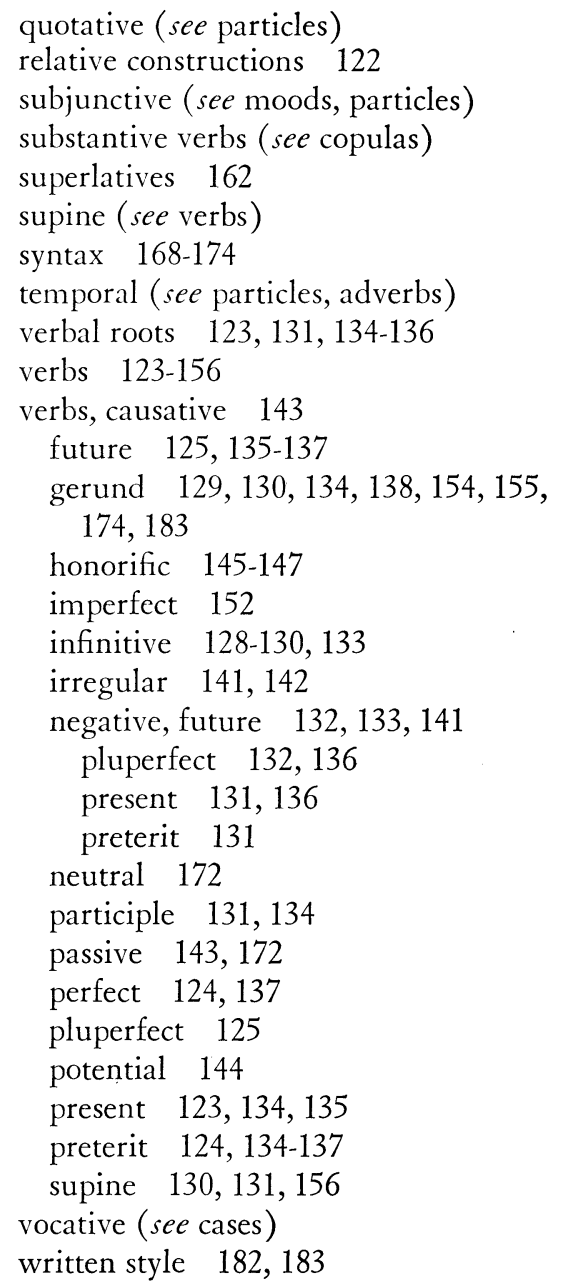




\section{Index to Grammatical Elements}

There follows a list of those elements which Collado describes in his grammar. To a certain degree I have regularized his morphophonological analysis. For example, the preterit permissive form, described by Collado as redomo after a preterit verb, is cross-listed as -ta redomo in order to bring together morphologically similar forms. All forms occurring in the text with the honorific gozaru, etc. are indexed as aru, etc. For example, the element found in aguenande gozaru 'I have not offered' will be indexed under -nande aru. As a general rule in this index items beginning with a hyphen are classified as endings, while the remaining items are particles.

The spelling used in this index is that of the original. Those readers more familiar with the modified Hepburn system of romanization, as reflected in Kenkyūsha's Dictionary, will find the following simplified chart of help. Syllables presented in Kenkyzusha as beginning with the following initial letters will have the corresponding spellings in Collado's grammar:

\begin{tabular}{|l|l|}
\hline$e=i e$ & $k=c a, q i, c u, q e, c o$ \\
$o=v o$ & $s=s a, x i, s u, x e, s o$ \\
\hline$h=f$ & $z=z a, j i, z u, j e, z o$ \\
$y=i$ & $t=t a, c h i, t c ̧ u, t e, t o$ \\
$w=v$ & $d=d a, g i, z z u, d e, d o$ \\
\hline
\end{tabular}

The citations are numbered according to their location in the translation and are limited to those places where the element is explained or used to demonstrate a grammatical point.

The following abbreviations are used:

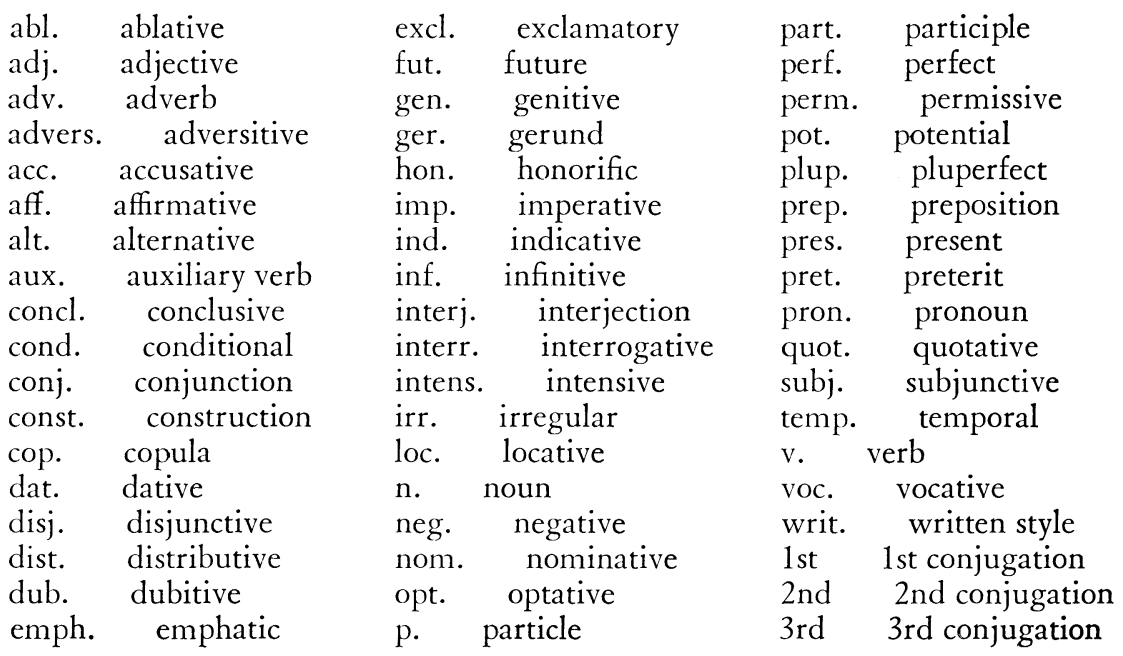




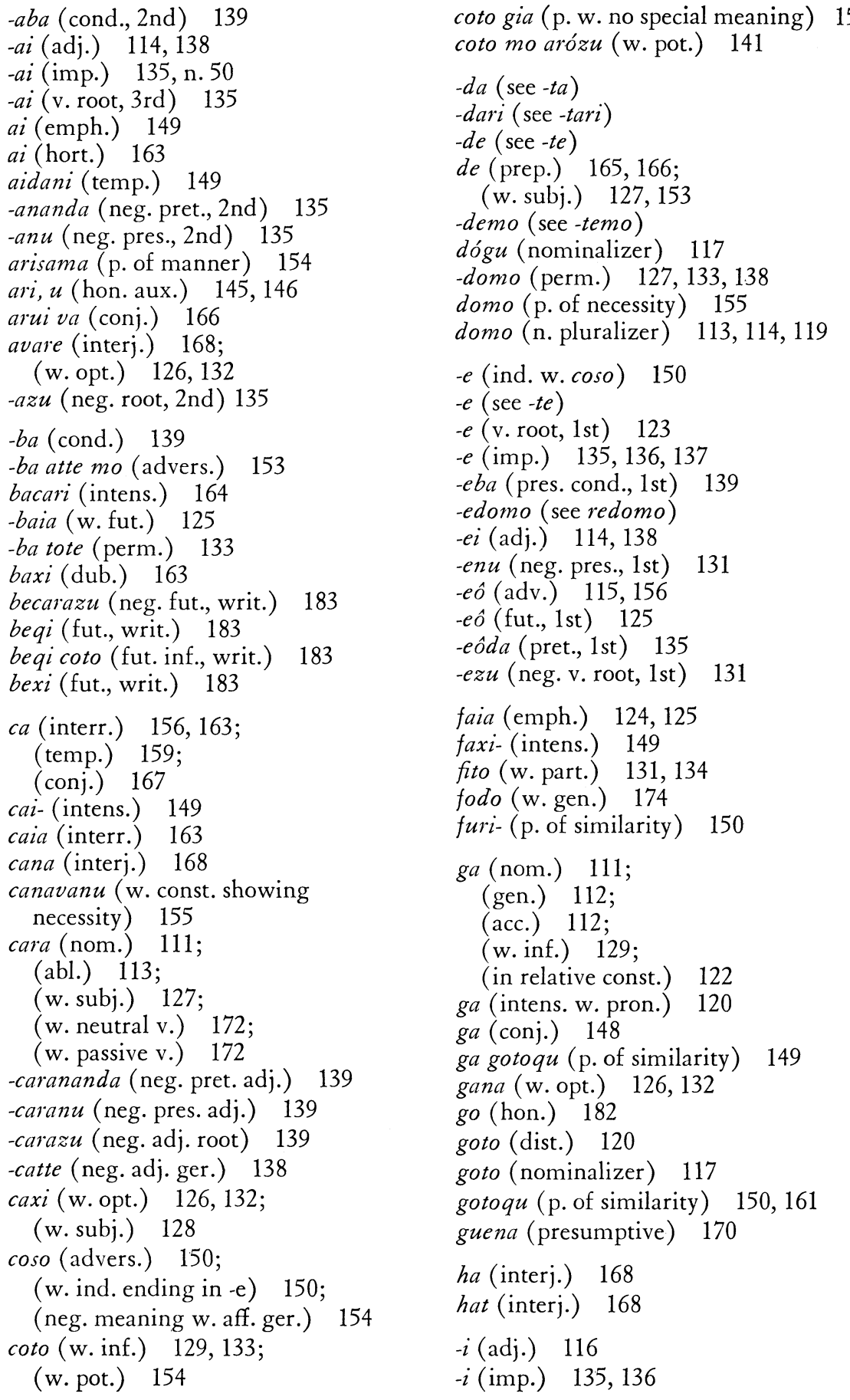


$-i$ (v. root, 2nd) 134

$-i$ (irr. v. root, 1st) 123

ia (excl.) 163

ia (interj.) 168

iai (excl.) 163

iara (interj.) 168;

(w. disj. const.) 167

iare (excl.) 163

-iasui (w. supine) 156

icani (voc.) 113;

(w. plurals) 113

- $i$ caxi (perm.) 129

-ide (neg. ger.) 134

-ide arózu (neg. plup. showing completed action) 137

-ide aru (neg. plup.) 132

-ide atta (neg. plup.) 132

-ide canavanu (ending showing necessity) 155

-idemo (neg. fut. perm.) 133, 154

-ide naranu (ending showing necessity) 155

-ide nochi (neg. ger.) 134

-ide va (ending showing necessity)

ie (acc.) 112;

(dat.) 112;

(prep.) 165;

(w. subj.) 127

ie (w. neg. possibility) 153

ie, uru (aux. of neg. possibility) 152

iei (interr.) 168

igo (w. subj.) 127

$-i j$ (adj.) 114,138

io (intens.) 163

io (imp.) 125

ió (p. of manner) 153

io caxi (w. opt.) 126

ióni (w. inf.) 129;

(w. quot.) 170

iori (nom.) 111;

(abl.) 113;

(w. inf.) 130;

(w. ger.) 174;

(w. comparative const.) 161;

(w. relative const.) 122

iori mo (w. comparative const.) 161

iori mo nao (w. comparative

const.) 161

- $i$ tomo (perm. adj.) 138

-iú (adv.) 115, 156 jibun (w. ger.) 130

$m a-$ (v. intensifier) 149

macari- (p. showing modesty) 149

made (prep.) 166

made gia (p. w. no special

meaning) 152

madeio (w. perm.) 128, 133;

(p. of confirmation) 152

mai (dist.) 120

mai (neg. fut.) 132

mai coto (neg. fut. inf.) 133

mai coto mo arózu (neg. fut. pot.) 141

maieni (w. neg. v.) 133

mai mono (neg. ger.) 134

mai mono vo (neg. opt.) 132

mai qereba (neg. subj.) 133

mai qeredomo (neg. perm.) 133, 155

mairaxi, u (hon. aux.) 147

mai tomo (neg. fut. perm.) 133

mai tote (neg. ger.) 134

maji (neg. fut., cf. mai) 132

maji qere (neg. cond.) 139

155

majiqu va (neg. cond.) 140

mamaio (w. perm.) 128, 133

maraxi u (hon. aux.) 145

mata (conj.) 166

mata va (conj.) 166

me (pejorative, w. pron.) 119,120

$m e$ (p. showing terminus of action) 117

me-(feminine) 114

mega (pejorative, w. pron.) $\quad 119,120$

mexi-(hon.) 147

mi- (hon.) 118, 182

mo (conj.) 166;

(dist.) 157;

(advers. w. ger.) 154;

(w. subj.) 128

mono (p. showing performer of action) 117

mono (w. part.) 131, 134;

(w. pot.) 141

mono de arózu (w. cond.) 141

mono vo (w. opt.) 126, 132

motte (emph.) 167

moxi (excl.) 163

moxi va (conj.) 166

-n (pres., writ.) 182

na (concl.) 163

na (neg. imp.) 132, 137 
na (adj.) 115, 117, 138

na caxi (neg. opt.) 132

-nagara (ger.) 155

nai coto mo arózu (neg.

pot. w. adj.) 141

-naide (neg. ger.) 134

-naide cara (neg. ger.) 134

-naidemo (neg. perf. perm.) 133

nama (p. showing incomplete action) 148

-nanda (neg. pret.) 132

-nanda coto (neg. pret. inf.) 133

-nanda mono (neg. ger.) 134

-nanda mono de arózu (neg. perf. pot.) 141

-nandaraba (neg. perf. cond.) 139

-nanda reba (neg. perf. subj.) 133

-nanda reba tote (neg.

perf. perm.) 133

-nanda redomo (neg. perf. subj.) 133

-nanda ritomo (neg. perf. perm.) 133

-nanda to (neg. perf. inf.) 133

-nande aru (neg. plup.) 132

-nande atta (neg. plup.) 132

-nanzzu ró (neg. perf. pot.) 141

-naraba (cond.) 139

naranu (w. const. showing necessity) 155

nari, $u$ (pot. aux. w. adj.) 141

nasare, uru (hon. aux.) 145

na...so (neg. imp.) 132, 137

-neba (neg. subj.) 132

-neba tote (neg. perm.) 133

-nedomo (neg. perm.) 133

negavacu va (w.opt.) 126, 132

ni (dat.) 112;

(abl.) 113;

(prep.) 164, 165;

(w. ger.) 130, 134;

(w. cond.) 138;

(w. subj.) 127;

(w. supine) 130,131;

(w. passive v.) 172;

(adv. form of $n a$ ) 121

ni iotte (prep.) 164;

(w. indefinite pron.) 121

ni itatte (prep.) 165

ni tai xite (prep.) 164

ni tçuite (prep.) 164;

(w. inf.) 130

ni totte (prep.) 165 niva (w. cond.) 139

ni voite va (prep.) 165;

(w. cond.) 139, 140

ni xitagatte (prep.) 165

ni xitagóte (see ni xitagatte)

ni xite (w. ger.) 130, 138

no (nom.) 111;

(gen.) 112;

(w. quote.) 171;

(to form adj.) 114;

(in relative const.) 122

nó (p. of confirmation) 163

nochi (w. subj.) 127

no gotoqu (prep., dialect) 166

no iori (prep.) 165

-nu (neg. pres., 1st) 131

-nu madeio (neg. pres. perm., 1st) 133

-nu maie ni (w. aff. meaning) 151

-nu mamaio (neg. pres.

perm., 1st) 133

$o$ (form of $v o$ after $n) \quad 171$

-ó (pres., 3rd) 135

-ó (fut., 2nd) 135;

(fut. imp., 2nd) 135

-ó (adv.) 115, 156

-ô (pres., 3rd) 136

-ô (fut., 1st) 125;

(fut. imp., 1st) 125

-ô (adv.) 115, 156

-ô coto (fut. inf., 1st) 129

-ô coto mo arózu (fut. pot., 1st) 141

-óda (pret., 2nd) 134

-ôda (pret., 2nd) 134

-ô fito (fut. part., 1st) 131

-oi (adj.) 114, 138

-oi (v. root, 3rd) 135

-ô mono (fut. part., 1st) 131

-ô ni (ger., 1st) 130

-ô tame (ger., 1st) 130

-óte (adj. ger.) 138

-ôte (adj. ger.) 138

-ô to (fut. inf., 1st) 129

-ô toqi (fut. subj., 1st) 127

-ô tote (ger., 1st) 130

-ó xite (adj. ger.) 138

-ôzu (fut., 2nd) 135

-ôzu (imp., 1st) 125

-ôzu mono vo (perf. opt., 1st) 126

-ôzure (fut., 1st, w. coso) 151

-ôzuru (fut., 2nd) 135 
-ôzuru (fut., 1st) 125

-ôzuru coto no saqi ni

(plup. subj., 1st) 128

-ôzuru ni (plup. subj., 1st) 128

-ôzuru tocoro ni (plup. subj., 1st) 128

-ôzu tomo (fut. perm., 1st) 128

qere (p. of confirmation) 150

qereba (w. subj.) 133, 138

qeredomo (w. perm.) 133, 139

-qi (adj.) 116

qiri,u (emph. aux.) 149

-qu (adj. root) 138

-qu tomo (adj. perm.) 138

-quva (adj. cond.) 139

-qu xite (adj. ger.) 138

ra (pluralizer) 113, 114, 118, 119

-raba (cond.) 139

-rare,uru (pot., 1st) 144;

(hon., 1st) 145;

(passive, 1st) 143

-re, uru (pot., w. 2nd \& 3rd) 144;

(hon., w. 2nd \& 3rd) 145, 147;

(passive, w. 2nd \& 3rd) 143

-re (pret. ending after coso,

see-tare) 150

-reba (subj., 1st) 127

reba (w. perf. subj.) 132;

(w. cop.) 138

-redomo (perm., 1st) 128

redomo (w. perf. perm.) 133;

(w. inf.) 130;

(w. cop.) 138

-ri (alt.) 152

ritomo (w. perf. perm.) 128, 133

ró (pot.) 140

$-r u($ see $-u r u)$

sa (nominalizer for adj.) 117

sai (imp.) 126

saie (emph.) 150;

(w. cond.) 140;

(w. neg. const.) 150

sama (prep., dialect) 166

sama (temp.) 154

sama (hon.) 119

saqini (w. neg. v.) 151

saraba (conj.) 167

sareba sareba (conj.) 167

sari nagara (conj.) 167

satemo (inter $\mathrm{j}$.) 167 satemo satemo (interj.) 167

sate sate (interj.) 167

-saxe,uru (causative) 143

saxemaxi,u (hon. aux.) 145

-saxerare,uru (hon.) 146

só aru tocoro de (conj.) 167

sóna ( $\mathrm{p}$. of presumption) 170

-ta (pret., 1st) 124, 134, 136;

(w. adj. function) 116

-tacatta (pret. of -tai) 153

tachi (pluralizer) 113, 119

-ta coto (pret. inf., 1st) 129

-ta fito (pret. part., 1st) 131

-tagari,u (2nd \& 3rd person

deciderative) 153

-tai (deciderative) 153;

(w. imp. meaning) 126

-ta madeio (per. perm., 1st) 128

tamai,ó (hon. aux.) 145

-ta mamaio (perf. perm., 1st) 128

tame (prep.) 164;

(w. ger.) 130, 134

tameni (w. supine) 130

-ta mono (pret. part., 1st) 131

-ta mono de arózu (perf. pot., 1st) 141

-taraba (perf. cond., 1st) 139

-taraba iocaró mono va (perf. opt., 1st) 126

-tare (pret. ending w. coso) 150

-ta reba (perf. subj., 1st) 127

-ta reba tote (perf. perm., 1st) 128

-ta redomo (perf. perm., 1st) 128

-tari (pret. writ.) 183

-tari (alt.) 152

-ta ritomo (perf. perm., 1st) 128

-taró ni va (perf. cond., 1st) 139

-taró va (perf. opt.) 126

-tarózu (plup., 1st) 125

tate matçuri,u (humble aux.) 147

-ta to (pret. inf., 1st) 129

tatoi (w. perm.) 128

$-t c ̧ u$ (alt.) 152

tçui- (intens.) 148

-tçu ró (perf. pot., 1st) 140, 151

-te (ger.) 129, 130, 155, 183;

(inf.) 129

-te (part.) 131

-te aranu (neg. pret.,

completed action) 137

-te aró (fut., completed action) 137 
- te aró ni va iocaró mono vo

(perf. opt.). 126

-te arózu (perf.) 124, 137

-te atta (perf.) 124, 137;

(w. perf. subj.) 127

-te atta reba (plup. subj.) 127

-te cara (plup. subj.) 127

-te coso (w. neg. meaning) 154

tei (p. of manner) 154

-te igo (plup. subj.) 127

te mo (subj.) 128;

(w. advers.) 154

-te nochi (plup. subj.) 127

to (gen.) 112

to (conj.) 166

to (w. inf.) 129,133

to (quot.) 168;

(w. adv. of sound) 163

tocacu (disj.) 167

tocoro (w. subj.) 127;

(p. of completed action) 151

tocoro gia ( $\mathrm{p}$. w. no special meaning) 151

tocoro no (w. relative const.) 122

-tomo (w. perm.) 128, 133, 138, 150

to mo (quot.) 170

-tó mo nai (neg. of -tai) 153

toqi (w. subj.) 127;

(w. pret. imperfect) 152

tori-(intens.) 149

tote (w. perm.) 128, 133;

(w. ger.) 134

to tomo ni (prep.) 165

to xite (w. ger.) 130

-tta (pret., 2nd) 134

$-u$ (pres., 2nd) 134

-u (adv.) 115, 156

-ú (pres., 3rd) 136

uchi-(intens.) 149

-ui (adj.) 115, 138

-ui (v. root, 3rd) 135

uie (prep.) 166

uie iori (prep.) 164

-unda (pret., 2nd) 134

-ureba (pres. cond.) 139

-uru (pres., 1st) 123

-uru fito (pres. part., 1st) 131

-uru iori (pres. inf., 1st) 130

-uru jibun (ger., 1st) 130

-uru madeio (pres. perm., 1st) 128 -uru mamaio (pres. perm., 1st) 128

-uru mo (fut. perm., 1st) 128

-uru mono (pres. part., 1st) 131

-uru ni (ger., 1st) 130

-uru ni tçuite (pres. inf., 1st) 130

-uru tame (ger., 1st) 130

-uru tameni (supine, 1st) 130

-uru tomo (fut. perm., 1st) 128

-uru tote (ger., 1st) 130

-uru vo motte (pres. inf., 1st) 128

va (nom.) 111;

(acc.) 112;

(w. subj.) 127;

(w. inf.) 130;

(w. cond.) 139;

(w. other p.) 114;

(replacing other p.) 114;

(w. const. showing necessity) 155

$v a$ (p. of confirmation) 149

-vaba (cond., 3rd) 139

-vananda (neg. pret., 3rd) 136

-vanande aru (neg. pret., 3rd) 136

-vanande atta (neg. pret., 3rd) 136

-vanu (neg. pres., 3rd) 136

-vazu (neg. root, 3rd) 136

vo (acc.) 113;

(w. subj.) 127;

(w. neutral v.) 172;

(becomes $o$ after $n$ ) 171

vo- (hon.) 146, 182

vo- (masculine) 114

-vó (fut., 3rd) 136

voba (acc.) 112

voi- (intens.) 149

vo motte (prep.) 165;

(w. inf.) 130

von- (hon.) 118, 182

vôxe-(hon.) 147

-vózu (fut., 3rd) 136

-vózuru (fut., 3rd) 136

-xe,uru (causative) 143

xemaxi,u (hon. aux.) 145

-xerare,uru (hon.) 146

-xi (adj.) 116

xicareba (conj.) 167

xidai (prep.) 165

xite (w. neg. ger.) 131, 134

$x u$ (n. pluralizer) 113

-zaru (neg. pres., dialect) 131 
-zatta (neg. pret., dialect) 131

-zatta reba (neg. perf. subj. dialect) 131

zo (interr.) 156, 159, 163;

(temp.) 159;

(dub.) 162;

(intens.) 162

$-z u$ (neg. v. root, 1st) 131 $-z \tilde{u} b a$ (neg. cond.) 139

-zumba (see züba)

$-z u$ tomo (neg. perf. perm.,

1st) 133

-zu va (neg. cond.) 139

-zu xite (neg. ger., 1st) 131, 134

$-z z u$ (alt.) 152

-zzu ró (perf. pot.) 140, 151 



\section{UNIVERSITY OF KANSAS \\ Center for East Asian Studies \\ International Studies, East Asian Series}

\section{RESEARCH SERIES}

1. Goodman, Grant K.

Davao: A Case Study in Japanese-Philippine Relations. $\quad \$ 4.50$

2. Goodman, Grant K., Compiler

The American Occupation of Japan: A Retrospective View. $\$ 2.50$

3. Schwarz, Henry G.

Liu Shao-Ch"i and "People's War": A Report on the Creation of Base Areas in 1938 . $\$ 2.50$

4. Wickberg, Edgar, Compiler

Historical Interaction of China and Vietnam: Institutional and Cultural Themes. $\$ 2.50$

5. Akashi, Yoji

The Nanyang Chinese National Salvation Movement, 1937-1941. $\$ 6.00$

6. Lee, Chae-Jin

Communist China's Policy Toward Laos: A Case Study, 1954-67. \$5.00

7. Sheng, Yueh

Sun Yat-sen University in Moscow and the Chinese Revolution: A Personal Account. $\$ 8.00$

8. Verdu, Alfonso

Dialectical Aspects in Buddhist Thought: Studies in Sino-Japanese Mahāyāna Idealism. $\$ 15.00$

9. Spear, Richard L.

Diego Collado's Grammar of the Japanese Language. $\$ \$ 0.00$

\section{REFERENCE SERIES}

1. Huang, C. C.

A Modern Chinese-English Dictionary for Students. $\$ 12.50$

2. Lindquist, Harry M., and Meyer, Roger D., Compilers

Concordance of Proper Nouns in the Five-Volume English-Language Selected Works of Mao Tse-tung. $\$ 3.00$

Sole distributors in the USA \& Canada

Paragon Book Gallery, Ltd.

14 East 38th Street

New York, N.Y. 10016 